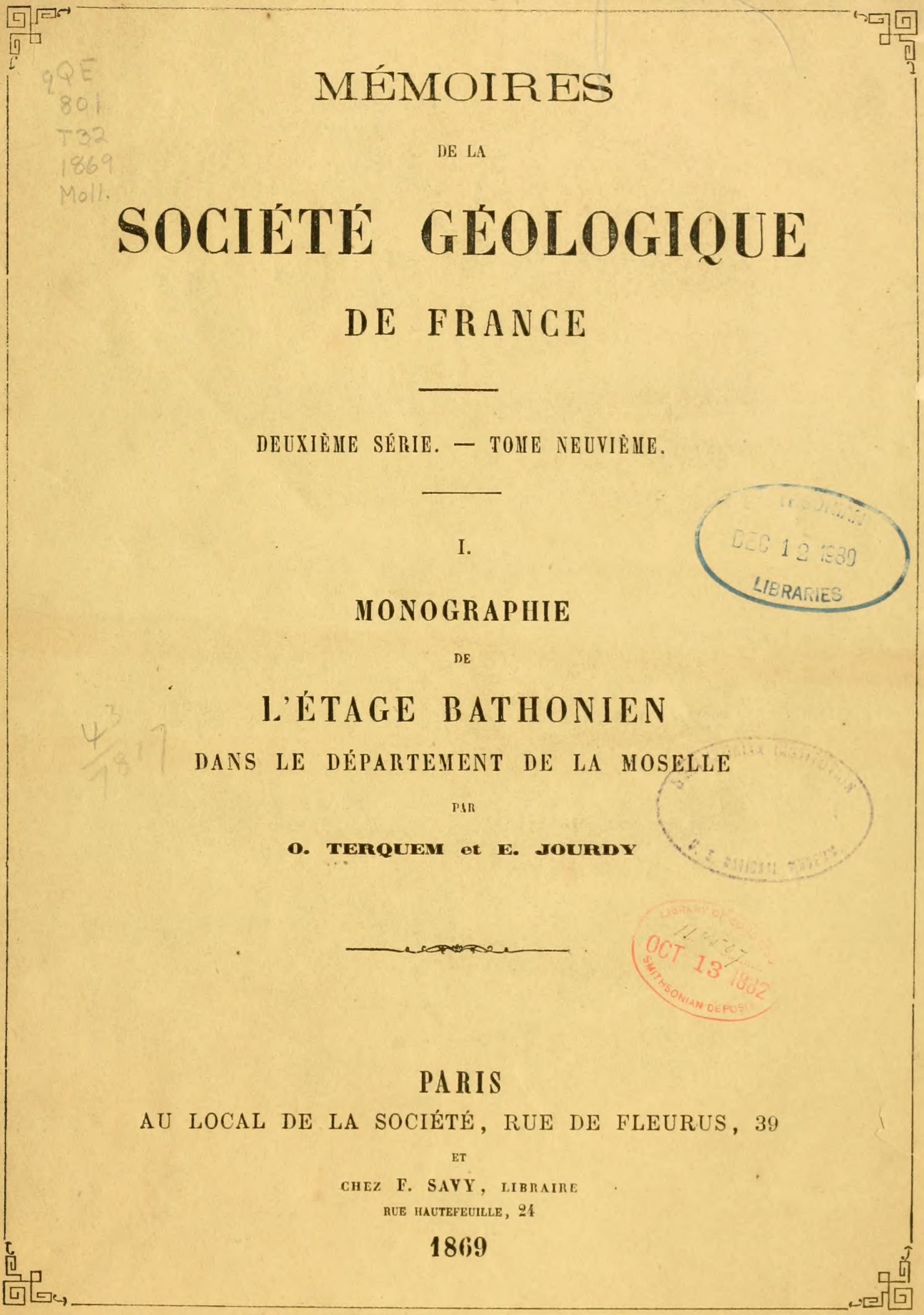





\section{I.}

\section{MONOGRAPHIE DE L'ETAGE BATHONIEN \\ DANS LE DÉPARTEMENT DE LA MOSELLE, \\ PAR}

MM. O. TERQUEM

ET

E. JOURDY.

\section{PREMIÉRE PARTIE.}

\section{STRATIGRAPHIE ET PÉTROGRAPHIE.}

\section{PREMIÈRE SECTION.}

\section{ROCHES DE L'ETAGE. - SA FAUNE. - SES DIVISIONS.}

La description des roches de l'étage avait déjà été entreprise dans le texte accompagnant la carte géologique du département (1868); mais ce travail prématuré sur le sujet que nous traitons, méritait une étude suffisamment complète pour connaitre les différents niveaux auxquels se trouvent les fossiles. Une nouvelle description stratigraphique nous a paru indispensable pour présenter ces terrains d'une facon plus vraie et plus complète.

Un résumé sur les particularités de la faune suit la description stratigraphique; il est extrait des tableaux qui se trouvent à la fin de la description paléontologique et destiné à mettre en lumière les faits généraux et caractéristiques des différentes zones de l'étage. De cette double description résulteront la connaissance des affinités caractéristiques ou distinetives de toutes les couches de l'étage et leur place naturelle dans la série des couches jurassiques.

Soc. GÉor. $-2^{0}$ sÉrie. T. IX. - Mém. no 1. 
PREMIERE ZONE. - ZONE A AMMONITES SUBFURCATUS.

$1^{\circ}$ Marnes de Longwy. - Quelle que soit la facon dont se termine le calcaire à Polypiers, le Bajocien est surmonté par une couche marneuse renfermant l'Ostrea acuminata avec plus ou moins d'abondance. Ces marnes sont sableuses dans certains endroits, très-argileuses dans d'autres; elles contiennent des petits banes de calcaires jaunes formés d'oolithes miliaires, qui deviennent de plus en plus abondants à mesure qu'on s'éloigne du calcaire à polypiers, et qui relient ces marnes à la couche calcaire qui les surmonte. Quand elles sont très-argileuses (sur les glacis de Longwy), elles présentent une couleur jaune ou bleue très-foncée, ce qui les fait distinguer très-nettement du calcaire à polypiers; certains bancs sont pétris d'Ostrea acuminata et présentent une faune assez riche. Quand elles sont sableuses (près de Gravelotte) elles ont une couleur grise, et leur séparation du calcaire à polypiers est peu nette; les fossiles y sont extrèmement rares ; c'est à peine si on y rencontre quelques Ostrea acuminata, qui y sont là longues et de grande taille.

L'épaisseur de cette couche est difficile à déterminer exactement à cause de la facilité avec laquelle les calcaires subordonnés se relient aux calcaires de Jaumont (couche supérieure); il est probable qu'elle ne dépasse pas 10 mètres.

Cette couche est rarement visible sur le terrain; cependant elle a une importance séricuse, à un autre point de vue : elle alimente un grand nombre de villages par les eaux qu'elle fournit sur la zone très-étendue le long de laquelle elle surmonte le Bajocien (MM. Dargnies et Barré).

C'est cette couche à laquelle M. Jacquot avait donné le nom de couche à Ostrea acuminala (Géologie de la Moselle); cette dénomination manque de justesse, attendu qu'on ne peut choisir, pour caractériser une couche, le nom d'une espèce qui est abondante à un grand nombre de niveaux différents. Les environs de Longwy en donnent une bonne coupe, où l'on peut saisir très-facilement le passage du Bajocien au Bathonien; car, lả, le calcaire à polypiers est représenté tantôt par une roche oolithique miliaire blanche sans fossiles, tantôt par des marnes sableuses et grises séparées en lits minces par des banes horizontaux de Polypiers; l'apparition de la roche jaune où pullule l'Osirea acuminata, donne pour la base du Bathonien un niveau parfaitement déterminé.

$2^{\circ}$ Calcaires oolitiilques mliares de Jaumont. - Calcaires formés d'oolithes généralement miliaires avec des débris de coquilles mélangés dans des proportions variables. " Dans la partie méridionale du département, l'élément oolithique domine ou existe seul, la pierre y est blanchàtre; presque partout ailleurs, il y a mẻlange; souvent mème les oolithes sont très-rares et les débris de coquilles constituent la masse principale de la roche; la couleur est alors jaune.

* Dans les bancs tout-à-fait supérieurs, on rencontre habituellement des 
oolithes blanches plus grosses que celles que l'on trouve dans Ia région inférieure.

a L'assise est done presque exelusivement calcaire; toutefois on y trouve sur quelques points des intercalations noirâtres ou bleuâtres remplies de grosses oolithes. Dans les environs de Metz, elle n'a pas plus de 13 à 20 mètres de puissance; mais ei e est plus développée dans la région de la Chiers où son épaisseur atteint de 25 à 30 mètres. Un des caractères les plus essentiels de l'oolithe de Jaumont a est de présenter des strates obliques aux plans de séparation des banes», (Jacquot, Géologie de la Moselle, p. 239.)

L'auteur que nous citons avait depuis longtemps reconnu la constance de cette couche sur une grande étendue; la facilité avec laquelle on peut la retrouver en fait la couche la plus caractéristique du Bathonien. De plus elle donne lieu, dans un grand nombre de localités, à l'exploitation d'une pierre de taille connue dans toute la Lorraine.

Les fossiles que renferme cette couche sont le plus souvent brisés ou agglomérés et donnent lieu à de fausses stratifications, comme s'ils avaient été charriés par des courants violents et variables.

Les deux conches piécédentes sont tellement unies, que les marnes de Longwy paraissent être des couches marneuses intercalées dans les premiers bancs du calcaire de Jaumont. Leur réunion forme la première zone.

Cette zone n'affecte pas de forme topographique bien spéciale; généralement visible sur le bord du plateau qui domine la Moselle, elle suit les allures du Bajocien qui, comme elle, se compose presque entièrement de couches calcaires; sur un assez grand nombre de points, au bord de la vallée de la Moselle, elle se sépare nettement de la deuxième zone, dont la base formée de couches meubles a cédé aux érosions, tandis que le calcaire de Jaumont avait plus de cohésion pour y résister; elle forme ainsi une sorte de corniche et sa crète donne les points les plus élevés du terrain Bathonien, dont les couches, plongeant à l'Ouest dans toute leur étendue, forment une sorte de plan incliné peı mouvementé et coupé par les vallées tributaires de celle de la Moselle.

\section{DEUXIÈNE ZONE. - ZONE A AMMONITES PARKINSONI.}

3. Marnes de Gravelotte. - Ces marnes sont colorées de différentes nuances pour une même localité ; et cette coloration est toujours assez vive quand le bleu s'y montre.

Elles renferment constamment une grande quantité de grosses oolithes ferrugineuses a de formes ellipsoïdales, qui ont pu être comparées à des grains de froment. On y trouve quelques roches bien agrégées, constituant 
des couches, et le plus souvent des lits de rognons juxtaposés. Ce sont des calcaires, les uns grenus et terreux, les autres légèrement saccharoïdes, offrant en général une grande ténacité; ils sont grisâtres ou brunâtres, en général pétris de fossiles et formant de véritables lumachelles; quelques-uns présentent un noyau central de couleur bleue comme les argiles, au milieu desquelles ils sont intercalés, et contiennent beaucoup d'oolithes aplaties." (Jacquot, loc. cit. page 261.) Les calcaires qui divisent la masse de ces argiles forment des amas variables suivant les localités; ils enlèvent beaucoup de l'imperméabilité aux marnes; grâce à eux, les vallées qui mettent la couche à nu sont loin d'être marécageuses comme le deviennent celles qui sont situées sur les marnes de la troisième zone, tout en permettant l'existence de sources abondantes.

La faune de ces marnes est en général très-riche; c'est à ce niveau stratigraphique qu'appartient le gisement des Clapes, près de Fresnois, où se trouvent des fossiles d'une conservation merveilleuse. Ces marnes ont environ 20 mètres d'épaisseur.

M. Jacquot donne à cette couche le nom de couche à Ostrea costata; cette dénomination est mauvaise, par la raison que ce fossile se trouve déjà dans les marnes de Longwy et est assez commun à plusieurs niveaux supérieurs (1).

$4^{\circ}$ Calcaires a points ocreux de Vernéville (Dargnies). - Calcaire gris, à cassure écailleuse, parsemé d'une multitude de points ocreux; ce calcaire ne renferme pas de fossiles et ne se trouve que sur certains points du département; mais la façon constante dont il se superpose aux marnes de Gravelotte permet de le considérer comme une couche distincte ; il se trouve surtout aux environs de Vernéville.

5 o Calcares a oolithes canvabines de Gravelotte. - Ces calcaires sont généralement blanes et formés d'une multitude d'oolithes cannabines, plus ou moins soudées entre elles; à Gorze, à Gravelotte, à Conflans, elles sont presque complétement désagrégées, tandis qu'aux environs de Vernéville, elles donnent lieu à une masse compacte.

Ces calcaires, quoique d'un blane généralement très-franc (surtout à la base), renferment des couches colorées en jaune avec des débris de coquilles méconnaissables; à la partie supérieure, le blane se ternit et tourne au gris avec des nuances pâles et variées. Cette couche renferme généralement des fossiles qui se trouvent tous dans les marnes de Gravelotte, et sont groupés identiquement de la même manière jusqu'à son sommet, sans que la moindre variation ne se produise dans les caractères des espèces.

$6^{\circ}$ Calcaires a oolithes mulaires du Grand-Failly. - Calcaires formés d'oolithes

(1) La petite huître en question n'est pas le véritable 0 . costata, mais une espèce trèsvoisine nommée $\boldsymbol{O}$. Gibriaci, Mart. 
miliaires d'un blanc crayeux ou jaunattre se délitant facilement à l'air ; les couches sont parfois séparées par de très-minces lits argileux contenant des concrétions poreuses très-légères et colorées vivement par des oxydes de fer et de manganèse; ces calcaires ne présentent pas de traces de fossiles.

Ces trois dernières couches calcaires ne s'observent pas partout superposées l'une à l'autre; même en mettant de côté la couche à points ocreux qui est peu constante, les deux autres sont susceptibles de variations intéressantes. Au sud du département, le calcaire à oolithes cannabines parait représenter à lui seul toute la partie calcaire de la deuxième zone; son épaisseur y varie de 10 à 20 mètres; non loin de Conflans, sur la rive droite de l'Iron, le calcaire à oolithes miliaires s'intercale dans les couches supérieures du calcaire à oolithes cannabines; au Nord du département, les oolithes miliaires paraissent exister seules (Dargniès) (1).

Ces trois couches calcaires sont en général dépourvues de fossiles; Ie calcaire cannabin seul en présente à sa base et à son sommet; tous ces fossiles appartiennent aux mêmes espèces que ceux des marnes de Gravelotte sans aucune variation mème insignifiante et avec une identité parfaite de distribution; leur épaisseur moyenne est d'environ 30 mètres.

$7^{\circ}$ Marnes et Calcaires marneux du Jarnisy. - Ces calcaires, bien différents des précédents, sont terreux au lieu de se faire remarquer par l'abondance des oolithes qui, pour la plupart des calcaires inférieurs, forment presque exclusivement la roche. Cependant, vers la partie supérieure, ils contiennent une couche oolithique (Pierre de Friauville de M. Barré); les oolithes y sont fines en général, intermédiaires de grosseur oolithes miliaires et les oolithes cannabines; elles y sont mélangées à des débris de coquilles indéterminables. Mais cette couche paraît très-instable; son épaisseur, assez faible en général, se réduit souvent à être insignifiante, et mème plusieurs de ces bancs sont formés par les calcaires ordinaires dans lesquels se trouvent disséminées quelques rares oolithes jaunes qui se séparent facilement de la roche. Ces calcaires oolithiques sont ordinairement colorés d'une teinte blanchâtre, tranchant un peu sur la couleur très-terne des calcaires dans lesquels ils sont compris.

Quant à ces derniers calcaires, qui forment la presque totalité de la couche, ils sont terreux, d'un gris terne, souvent bruns, sans oolithes. Ils sont stratifiés en banes peu épais qui se délitent en petits fragments polyédriq̨ues, irréguliers; leur cassure est plane et grenue. A la base, les calcaires sont plus abondants, renferment peu de fossiles; au sommet, les marnes dominen (quelques parties présentent de petits lits contenant une grande quantité d'or aes cannabines), avec une faune plus riche et passant à la couche supérieure, qui est tout-à-fait argileuse.

Quelques bancs ealcaires de cette couche portent des tiges dichotomes à leur

(1) Enfin dans la Meurthe on retrouve le facies des environs de Longuyon (Dargnies). 
surface, qui est rugueuse et comme saupoudrée d'un sable fin. L'importance de cette observation ressortira dans l'étude détaillée de la faune de ces calcaires.

Le faciès minéralogique de cette couche s'éloigne beaucoup des couches inférieures de la zone, même en tenant compte du calcaire oolithique qu'elle renferme parfois; la dissemblance est d'autant plus frappante que la pétrographie la rapproche complétement des couches de la zone supérieure.

Mais sa position dans la classification est indiscutable, si on tient compte du caractère domné par les fossiles, le seul qui puisse ici servir de guide. Les fossiles y sont les mêmes que dans la $3^{\mathrm{e}}$ zone. De plus, la jonction de cette couche aux calcaires cannabins doit être des plus intimes comme l'annonce un fait paléontologique remarquable: les fossiles des calcaires cannabins pénètrent en certains endroits de la roche des calcaires du Jarnisy et ne s'éteignent qu'à quelques décimètres plus haut que la jonction des eouches (environs de Jarny).

Cependant, tout en reconnaissant que la faune de cette couche la place dans la $2^{\mathrm{e}}$ zone, nous ferons remarquer que ses analogies avec la $3^{\mathrm{e}}$ zone, qui sera décrite plus loin, sont très-importantes au point de vue des fossiles et surtout à celui des roches. La pétrographie consultée seule, classerait cette couche d'emblée dans la $3^{e}$ zone; de plus, sa faune elle-mème comprend quelques espèces qui commencent là leur apparition par des individus peu nombreux pour pulluler dans la $3^{\mathrm{e}}$ zone; enfin, un grand nombre des espèces de la $2^{\circ}$ zone, en traversant les calcaires du Jarnisy, présentent des variétés du plus haut intérêt.

Cette couche est done des plus remarquables, d'abord par son rôle de transition entre la $2^{e}$ et la $3^{\text {e }}$ zone, puis par les particularités paléontologiques qu'elle présente. Ce double sujet sera traité plus en détail dans la discussion de la faune propre à chaque zone et dans la description des espèces. Cet ensemble de marnes et de calcaires, plutôt argileux que calcaire, couvre trèsnettement la surface du Jarnisy sur le sommet des collines formées par les calcaires oolithiques; puis, à mesure qu'on s'avance plus à l'ouest, on les voit former le sous-sol jusqu'à ce qu'ils disparaissent sous les marnes de la zone supérieure. Cette couche a environ 25 mètres d'épaisseur.

$8^{\circ}$ Marnes noires, arglleuses, a Ostrea Knorril de Friauville. - Cette couche a une faible épaisseur et paraitrait insignnifiante si une observation attentive ne faisait remarquer son importance à plusieurs points de vue.

Ses caractères pétrographiques la séparent des deux zones entre lesquelles elle est placée; elle est très-brune, très-argileuse, ne renferme que de trèsminces et très-petites plaquettes d'un calcaire très-argileux.

Elle est très-remarquable par l'abondance presque exclusive de l'Ostrea acuminata et surtout de l'Ostrea Knorrii, fossiles qui sont rares au sommet de la $2^{\mathrm{e}}$ zone, et encore plus rares à la base de la $3^{\mathrm{e}}$ zone. Le sommet des calcaires 
du Jarnisy devient très-marneux et passe insensiblement à cette couche dont la place dans la $2^{\mathrm{e}}$ zone est alors établie.

Enfin, au point de vue topographique, cette couche sert de point de repère pour la détermination sur le terrain, du sommet de la $3^{\mathrm{e}}$ zone. Comme ces marnes sont très-grasses, elles donnent lieu en certains points à de petits prés humides qui suivent quelquefois la limite de la $2^{\circ}$ et de la $3^{\circ}$ zone; dans d'autres endroits elles donnent lieu à des ressauts, peu accentués, il est rrai, mais qui délimitent nettement les buttes formées par la zone supérieure.

\section{TROISIËME ZONE. - ZONE A AMMONITES QUERCINUS.}

9. Maryes de Conflans. - Au-dessus des marnes noires à Ostrea Knorrii de Friauville, on voit reparaitre un massif de marnes brunes, un peu sableuses, quelquefois noires et un peu argileuses renfermant quelques banes mal stratifiés, tendres, à demi délités d'un calcaire brun avec taches bleues; les marnes el les calcaires sont identiques aux marnes et aux calcaires du Jarnisy, les bancs calcaires sont très-désagrégés à la base de la zone; mais, vers le milieu et non loin du sommet, ils forment des bancs réguliers bien stratifiés, mème nieux stratifiés que les calcaires du Jarnisy, ils ont de plus une couleur un peu claire (Béchamp).

Certains bancs calcaires présentent la particularité que nous avons signalée dans les banes supérieurs de la deuxième zone (pierre de Friauville). Ils deviennent oolithiques et plus durs (Puxe, Béchamp), ce qui donne parfois à l'orne des berges un peu roides au lieu des pentes douces formées par les calcaires très-marneux.

Les fossiles sont très-communs à la base, ce qui constraste nettement avec la couche à Ostrea Knorrii qui est très-pauvre, à part son fossile caractéristique. Mais plus on s'avance, plus ils deviennent rares et finissent mẻme par s'éteindre à une certaine hauteur, c'est à pẹne si quelques débris d'huîtres se rencontrent vers le sommet de la zone (Puxe, ferme de Reuvron); les particularités de cette distribution seront traitées plus loin.

2. Marnes somes a Ostrea Kvorru, de Rouvres. - Ces marnes ressemblent complétement aux marnes à Ostrea Knorrii de Friauville qui couro in la $2^{\circ}$ zone, sinon qu'elles sont plus épaisses et plus argileuses. C'est la première couche du Bathonien qui se trouve tout entière dans la Meuse; cependant il est probable qu'elle doit se rencontrer encore dans la Moselle, dans le bois de Puxe, derrière Dompierre.

Elle acquiert dans la Meuse une certaine importance, elle occupe un bas-fond marécageux, très-large devant Rouvres, qui se poursuit jusqu'auprès de Spincourt; ce bas-fonds donne de nombreux étangs; le lit du ruisseau du 
Haut-Pont et celui de l'Ohain qui va se jeter dans la Chiers au delà de Longwy y sont compris.

Ces marnes couronnent la $3^{\text {e }}$ zone, exactement comme celle de Friauville couronnaient la 2" et ces deux couches, tellement identiques, sont éloignées de plus d'une lieue. On est bien certain que ces deux couches marneuses sont à des horizons différents, car les marnes de Rouvres, sur la limite de la Moselle, couronnent des collines dont la base renferme l'Ammonites quercinus, tandis que les marnes de Friauville sont surmontées par les couches qui renferment ce fossile; or, cette partie de la Lorraine ne peut renfermer aucun renversement; les failles, à la vérité, y sont nombreuses, mais tellement faibles qu'il est impossible, la plupart du temps, de déterminer leur direction.

La $3^{e}$ zone, vers son milieu, devient de plus en plus pauvre en fossiles; aux environs de Puxe, d'Olley, c'est à peine si l'on trouve quelques Gresslyes et quelques Rhynconnelles; enfin, vers son sommet réapparaissent quelques calcaires un peu plus compactes qu'à la base, renfermant encore l'Ammonites quercinus.

Mais on arrive ainsi à sortir complétement du département de la Moselle. Cependant pour donner line idée complète de l'étage dans tout son développement, nous dirons quelques mots de sa délimitation au contact de l'oxfordien ; la description ne sera que stratigraphique, car la faune ne change plus jusqu'aux marnes à Trigonia clavellata.

\section{QUATRIÈNE ZONE. - ZONE TRĖS-PEU FOSSILIFĖRE.}

Cette zone n'a pas pour nous une importance réelle, et il est bien possible qu'on la fasse rentrer plus tard dans la zone sous-jacente, car les rares fossiles que nous y avons trouvés sont ceux de la $3^{e}$ zone; seulement la grande rareté de ces fossiles succédant à la richesse des couches inférieures et la texture différente des calcaires du sommet nous ont paru dignes d'être signalées d'une façon spéciale, c'est-à-dire en isolant un peu les couches qui présentent ces particularités.

$1^{\circ}$ Calcaires terroux bruns de Rouvres. - Ces calcaires se présentent audessus des deuxièmes marnes à $O$. Knorrii exactement, comme les marnes calcaires de Conflans au-dessus des premières marnes noires, et la topographie du pays en avant de Rouvres, surtout sur le bord de la route, a quelque analogie avec les environs de Conflans, mais avec des reliefs moins accusés

Leur texture est celle des marnes subordonnées qui sont, du reste, identiques à celle des couches qui oceupent le milieu de la $3^{\circ}$ zone. Cependant en avant de Wareq, on y trouve de plus, dans les parties marneuses, de gros nodules de calcaires ovoïdes et de grosses concrétions siliceuses fendillées.

$2^{\circ}$ Calcaires oolithiques miliaires d'Étain. - Le passage des calcaires 
précédenis aux calcaires oolithiques se remarque à la ferme Rosa (entre Rourres et Wareq), où quelques couches marneuses donnent des fossiles malheureusement irop rares. Ces calcaires sont très-oolithiques, colorés en jaune, quelquefois assez foncé; ils donnent parfois des banes assez épais; mais à la surface du sol, ils sont séparés en dalles minces brisées dans la couche; leur cassure est plane et fait voir une foule de points brillants qui ne sont autre chose que des sections de zoophytes (Encrines ou pointes d'oursins) ainsi que des fragments de coquilles; ils ont un peu l'aspect de la dalle nacrée du Jura. Un assez grand nombre de bancs n'ont pas la texture oolithique, d'autres ne renferment que des oolithes dans leur pâte.

Ces calcaires sont fendus par de nombreuses failles qui dẻrangent fréquemment l'inclinaison des couches, et il est souvent difficile d'en reconnaitre l'ordre de superposition.

I. Buvignier a bien décrit les caleaires d'Étain et les considère, il est rrai, comme bathoniens (Géologie de la Meuse), sculement d'après ce qưil en dit, on est en droit de croire que la raison de cetle manière de voir consiste dans l'assimilation qüil fait entre les ealcaires d'Étain et les calcaires oolithiques miliaires de la 3 zone; quant aux couches marneuses qui, de Conflans à Étain, séparent ees deux couches calcaires, M. Buvignier les regarde comme oxfordiennes, de sorte que la limite entre l'Oxfordien et le Bathonien devient complètement fausse par la confusion de couches bien distincies; aussi ne faut-il pas s'étonner si on trouve réunies dans sa liste le Gryphoa dilatata et l'Ostrea Knorrii dont la présence simultanée n'a jamais pu être constatée dans une même couche, du moins e'est ce rue nous croyons.

\section{OXFORDIEN.}

Ayant ainsi critiqué l'empressement des auteurs à faire descendre l'0xfordien jusqu'au sein des couches les plus franchenent bathoniennes, nous avons voulu voir par nous-même quelle ótait la véritable limite de ces terrains. Nous ne l'avons pas vue en ce sens que nous n'avons pas touché le contact, et nous regrettons que cette constatation soil impossible; mais à quelques mètres au-dessus des calcaires d'Étain et de Wareq, nous avons vu des marnes feuilletées noires sans fossiles, pas même des foraminifères, aussi azoïques que les marnes irisées, renfermant de petits cristaux de sulfate de chaux et de petites conerétions calcaires fortement colorées en blare par du carbonate de chaux; on en voit une coupe à Buzy, sur la rive droite de l'Orme; au moulin de Blanzy, près d'étain, des marnes que nous supposons supérieures aux précédentes, renferment une srande quanité de Trigonia clavellata; l'aspect minéralogique est le mème.

Vers eet horizon, les étangs deviennent plus nombreux, surtout au Sud où la liegrion marneuse est plus étendue. Quand on eherche à reconnaitre la séric

$$
\text { Soc. GÉor. - } 20 \text { š́RIE. T. IX. - Mém. no } 1 .
$$


(N. I, P. 10.)

des couches oxfordiennes en s'avancant vers Étain et Verdun, on voyage dans un pays monotone, très plat, les quelques petits plis de terrain qui le traversent sont occupés par des ruisseaux où l'eau est assez abondante: de distance en distance, des étangs. Aucune coupe ne permet de se rendre compte des couches dout l'inclinaison à peu près identique à celle du sol, fait présumer qu'on monte peu dans la série verticale jusqu'aux collines en avant de Verdun. 
TABLEAU des couches que comprend le Bathonien.

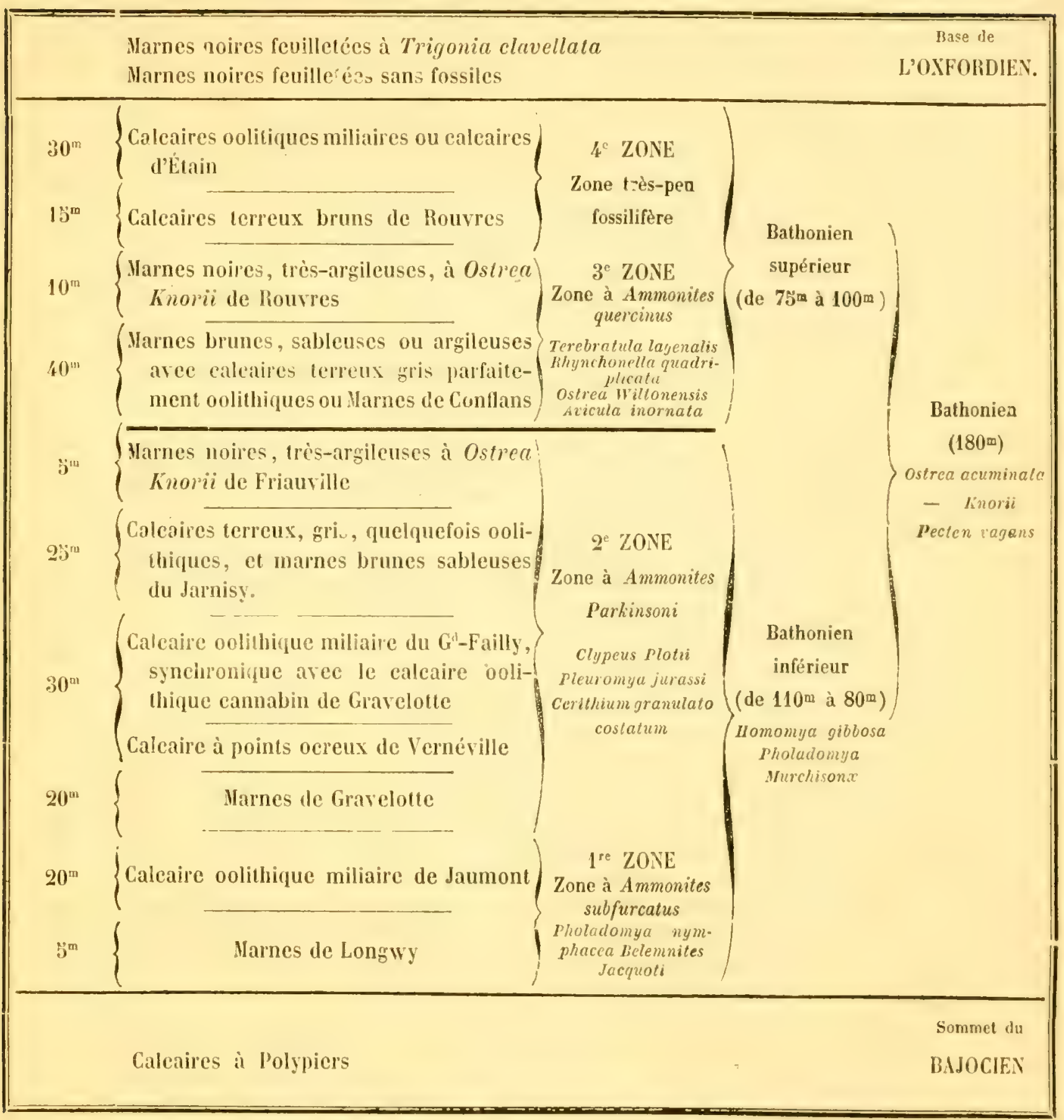




\section{FAUNES DES TROIS ZONES.}

Telle est la description des roches depuis le Bajocien jusqu'à l'0xfordien.

Se borner à donner l'énumération de ces différentes couches et à indiquer leur superposition, serait suffisant pour donner une idée exacte de leur stratiographie ; mais la connaissance de leur faune avec Ies particularités de distribution qui s'y trouvent peut seule les faire reconnaitre dans le temps et dans l'espace, c'est-àdire dans leurs relations avec les deux étages entre lesquelles elle sont placées, ainsi que dans leurs rapports géographiques avec les terrains du mẻme âge.

Pour se rendre comple du véritable caracière de la faune propre à chaque zone et des affiniiés entre elles de ces faunes partielles, il suffit de se reporter aux tableaux qui donnent pour chaque zone sa faune complète.

$1^{0}$ la première zone renferme 147 espèces parmi lesquelles on peut distinguer quatre catégories:

$1^{\circ}$ Espèces provenant du Bajocien.......... 46

$2^{\circ} \quad$ - spéciales à la première zone....... 34

$3^{\circ} \quad-\quad$ qui se trouvent aussi dans la $2^{\mathrm{e}}$ zone.. 100

$4^{\circ} \quad-\quad-\quad-\quad 30$

On voil que 46 espèces sur 147 sont bajociennes. La riche faune qui régnail depuis le Lias perd subitement la plus grande partie de ses espèces, au moment où apparaissent en quanticé énorme des espèces qui, comme l'Ostrea acuminata, doivent se propager sur une grande épaisseur. Ce fait indique une séparation dans les nouveaux dépós , e'est-à-dire l'apparition d'un nouvel élage.

$2^{\circ}$ La seconde zone renferme 218 espèces, narmi lesquelles on distingue encore :

$1^{\circ}$ Espèces provenant du Bajocien........... 47

$2^{\circ}$ - de la première zone............ 53

$3^{\circ} \quad$ - spéciale à la deuxième zone ........ 190

$4^{\circ} \quad$ - qui se trouvent aussi dans la $3^{\mathrm{e}}$ zone... 39

Les espèces qui du Bajocien montent jusqu'à la $2^{\mathrm{e}}$ zone, sont, à une ou deux près, les mêmes que celles qui, du Bajocien, passaient déjà à la $1^{\text {to }}$ zone.

Cette faune nomade n'a pas reç d'altération en passant de l'une de ces zones à l'autre; ce fait indique que, par rapport au Bajocien, ces deux zones forment un même groupe, première division de l'étage bathonien.

Les affinités de ces deux zones composant le groupe sont bien évidentes par la présence de cinquante-trois espèces qui leur sont communes, outre les quarantesept qui proviennent de plus bas, espèces dont la plupart sont des fossiles trèsabondants dans ces deux zones.

Le chiffre de 190 espèces caractérisant la $2^{\mathrm{e}}$ zone, parait au premier abord assez considérable pour isoler cette $2^{e}$ zone ; mais il est au fond beaucoup moins significatif si l'on a égard aux considérations suivantes: quand on fait des calculs de cette sorte pour se rendre compte autant que possible des aftinités des 
faunes entre elles, on est sujet à de nombreuses causes d'erreur grâce auxquelles les chiffres, pour être bien commodes, ne sont pas toujours la traduction fidèle des faits. Ainsi, pour le cas actuel, il est évident que les deux autres zones, n'offrant pas de gisement qu'on puisse comparer à celui des Clapes, le tableau de la faune de la $2^{\bullet}$ zone n'est pas davantage comparable à celui des deux autres dans lesquelles les espèces rares (ce qui est le cas de la majorité de ces 190 espèces) araient plus de chances d'ètre dérruites, ce qui a diminué d'autant la faune pour n'y laisser subsister que les espèces les plus abondantes. Aussi, dans le eas présent, doit-on, pour rendre les ehifires comparables, si non les réduire (car on ne peut pas connaitre la proportion à garder ) du moins attacher au sentiment une importance d'autant plus faible au nombre des espèces spéciales, qu'il se compose d'espèces rares provenant d'un gisement exceptionnel. Quand on rétablit ainsi le caracière véritable de la faunule spéciole à la $2^{e}$ zone comparée à la faunule qui réunit cette zone à la première, on éprouve, au contraire, unc hésitation à faire entre ces zones un coupure de quelque importance.

$3^{\circ}$ La $3^{e}$ zone contient 48 espèees ainsi distribuées :

$1^{\circ}$ espèces provenant du Bajocien..... 17

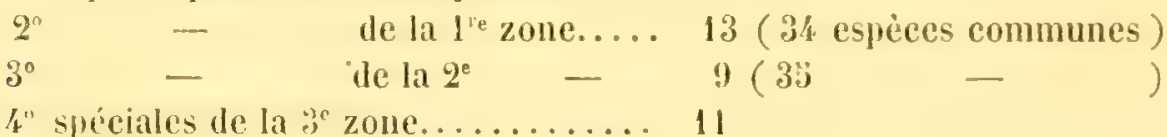

En arrivant à cette zone, la faune émigrée du Bajocien diminue subitemen! des deux tiers, chifre fu'il faut encore diminuer pour diverses considérations. D'abord, beaucoup de ces espèces sont nomades jusqu'à l'Oxfordien, puis toutes (sauf la Gresslya lumulaia) sont rares dans cette zone. D'où il résule que la '̉' zone doit être séparée du Baiocien d'une façon plus accentuée que les deux premières et entrer dans un second groupe isolé du premier. 'Hurmi les treize espèqui proviennent de la premiere zone (un peu moins du tiers de la faune) Ia plupart sont des espèces abondantes dans toute la série des couches précédemment étudiées. Ce chiflre, bien qu'un peu faible, montre que ces espèces nomades relient, par leur présence, les denx groupes dont nous avons parté, de manière à en faire les deax parties d'un même étage.

Ces treize espèces qui, de la première zone montent à la troisième, traversent également la seconde, excepté une ou deux qui sont rares dans les tro:s zones, ce qui montre encore que les deux groupes précédemment admis sont intimement liés entre eux. Enfin, des trente-cinq espèces communes aux $2^{\mathrm{e}}$ et $3^{\mathrm{e}}$ zones, neuf seulement apparaissent dans la deuxieme zone, et encore n'apparaissen -elles que dans les calcaires du Jarnisy. Cette couche est exceptiomnelle, et son a finité particulière, quoique peu prononée (9 espèces sur 48) avec la tioisiène zone, peut se rapprocher d'un fait décrit plus haut, eelui de l'identité de sa roche avec eclle de la troisieme zone.

Ainsi, d'une part la deuxième zone à elle seule n'offre aucun caractère spécial 
qui la rapproche de la troisième; de l'autre, l'ensemble seul des deux premières zones rapproche intimement les deux groupes formés, l'un par les deux premières, et l'autre par les deux dernières zones. Ces groupes sont, l'un la partie inférieure, de l'élage Bathonien, l'auśe, sa partie supérieure, chacun d'eux, étant composé de deux zones peu limiées entre elles, beacoup plus distinctes des deux autres. L'étage comprend done l'ensemble des couches comprises dans dans les quatre zones, e'est-à-dire 180 mètres envion d'épaisseur. Entre ces deux divisions, il est impossible d'en établir d'intermédiaires; aussi est-on en droit de conclure que la Moselle ne possède pas les couches à Terebratula cardium, Rhynchonella decorata, Rhynchonella Hopkinsi, Terebratula digona, $T$. coarctatu qui constituent la partie moyenne du Bathonien dans les pays où cet étage est plus complet. L'absence de ces couches si développées dans certaines contrées explique le caractère spécial de la couche appelée calcaire du Jarnisy, qui assista à l'émergement des dépôts inférieurs, pendant que se continuaient ailleurs les dépôts moyens. Cette lacune se manifeste dans la la couche en question: $1^{\circ}$ par les particularités stratigraphiques qui consistent dans la présence de liges dichotomes et de dalles saupoudrées d'une sorte de grès rugueux, indices de la proximité du rivage et dans la texture de la roche qui l'éloigne de celles de la deuxième zone pour se rapprocher de celle des la troisième zone. (Il n'y a pas de discordance de siratication, ce qui prouve que le mouvement a été lent. ) $2^{\circ}$ par les particulariés paléontologiques qui consistent dans l'apparition d'espèces qui deviendront moins rares dans la troisième zone et surfout dans les modifications éprouvées par beaucoup d'espèces qui plus bas n'offraient pas encore de variétés (ce qui prouve aussi que le sol émergé par intervalles assistait non à un renouvellement, mais à une variation de la faune locale). Ainsi done, les traits caraciéristiques des dépôts bathoniens de la Moselle et de la Neuse peuvent se résumer ansi : $1^{0}$ développement stratigraphique et paléontologique excessifs pour la partie inférieure; $2^{\circ}$ manque total de la partie moyenne; $3^{\circ}$ particularités du sommet de la partie inférieure correspondant à l'époque du dépôt de la partie moyenne; $4^{\circ}$ Développement stratigraphique complet avec développement paléontologique incomplet pour la partie supérieure. On verra que ces traits caractéristiques, dans l'étude du synchronisme, définissent le facies Vosgien 'opposé au facies Ardennais qui présente une autre lacune, et au facies Jurassien où le développement stratigraphique et paléontologique est normal pour tout l'étage. 


\title{
CHAPITRE II.
}

\author{
Synchronisme de l'étage avec les couches du même âge, \\ en Angleterre et dans le Nord-Est de la France.
}

\section{§ I. DU FULLER'S EARTH.}

L'étude de synchronisme qui suit la description de l'étage, a pour but de suirre ses modifications dans les diférents pays avoisinant la Moselle et ceux qu'on a pris comme types pour établir l'âge de ces différentes couches.

Nous commencerons par les couches d'Angleterre, qui renferment une partie de notre faune, non pas que les analogies de ces couches synchroniques soient des plus frappantes, mais parce qu'il a été longtemps d'usage de les prendre comme points de comparaison.

Il est bon de montrer une fois pour toutes, que les divisions anglaises ne peuvent en aucun cas être prises comme types, et que le terme de Fuller's Earth, sous lequel on désigne trop souvent la base du Bathonien, n'est pas applicable quand on le soumet à une analyse rationnelle.

Le Fuller's Earı, en somme, n'a jamais été l'objet d'une monographie pouvanı servir de terme de comparaison aux travaux du même genre. L'histoire des progrès de la géologie, qui résume les premiers travaux anglais, montre très-bien qu'on a souvent parlé du Fuller's Earth, mais qu'on ne l'a pas étudié suffisamment. Désirant nous trouver au courant de ce qu'on sait actuellement sur la paléontologie et la siratigraphie de cette couche, nous avons consulté ce qu'il y a de plus nouveau à ce sujet.

C'est d'abord un catalogue récent (1863) des fossiles du musée du géologie pratique de Londres, par MII. Huxley et Etheridge. L'habileté de ces paléontologistes est connue et surtout recommandée à tous les savants par une préface de M. Murchison. Tous les fossiles du Fuller's se retrouvent dans l'Inérior oolite ou le Great-oolite; il est complètement impossible, après cela, de définir cette couche par une faune spéciale capable de la faire reconnaitre. Dans la Moselle, au contraire, les couches du même âge, quoique offrant de nombreux passages, peuvent être caractériseés par un certain nombre de fossiles stationnaires et en général abondanis.

En second lieu, M. Ralph-Tate a bien voulu nous envoyer une description inédite du Fuller's; rien n'est done píus nouveau. M. Tate cite les districts où cette 
couche est bien développée (et ils sont assez peu étendus), et ceux oủ elle s'atrophie. En dehors de cette zone, la couche en question est bien difficile à reconnaitre. M. Morris suppose que ceriains sables du Northumberland sont de cette époque; M. Philipps a: aibue le même àze à des g.ès du Yorkshire. En un mot, en dehors d'une étendue restreinte, le Fuller's acquiert rapidement des caractères exceptionnels par la présence de grès, de sable, de lignites, de plantes, indices d'une influence cotière periurbatrice. 0 r, comme la mesure de ces anomalies est impossible à consiai r, ce qu'on peut faire de mieux, c'est de chercher à comparer les couches du Fu'ler's avec ce'les ọui sont mieux délimitées dans d'autres pays et non pas de suivre la méthode inverse.

Il y a long:emps déjà que N. Marcou l'a dit dans sa polémique avec M. d'Archiac: "On ne peut pas prendre pour types des couches jurassiques les dépôts d'Angleterre qui ont éié formés dins un ętuaire très-long et très-tortueux, le long duquel des dépôts continen aux sont venus à plusieurs reprises apporter des éléments qui ne sont plus comparables; au contraire, pour avoir une idée vraie des transformations de la faune au fond des mers, il faut s'éloigner du rivage et prendre les couches jurassiques dans les pays où les influences de la côte ćtaient annulées. ” Le reste de cette étude de synchronisme montrera comment on peut opérer ces iecherches.

A l'appui de cetce manière de voir, H. Tate cite l'opinion de M. Ramsay. D'après ce savant, il est impo sible que le Fuller's Earth contienne des "espèces spéciales à cette couche qu'il considère comine un accident produit par l'irruption d'un dépôt de vases au moment où commencait le dépôt du Great-oolithe; alors apparurent d'une manière éphémère certaines espèces vasogènes vivant antérieurement, espèces qui sont venues se fixer au milieu d'une colonie différente et dont la majorité disparut lors du rétablissement du dépót normal. M. Tate trouve cette opinion trop exclusive, mais la raison qu'il en donne est démonstrative contre l'Anglomanie: "Car, dit-il, le Fuller's Earth n'est pas encore suffisamment connu; certains banes rapportés à cette couche n'en font pas partie et inversement; enfin la plus grande partie des espèces qu'on y rencontre n'ont pas été décrites.

Voila donc ce criterium stratigraphique! Personne ne le connait. Pour nous, nous reconnaissons que la plus grande partie du Bathonien inférieur renferme les fossiles cités dans les listes du Fuller's, tout en pensant qu'il n'y a pas un synchropisme rigoureux à établir. L'éclaireissement de cette question dépend maintenant des savants Anglais auxquels nous avons fourni un point de comparaison.

§ II. M. D'ARCIJIAC.

Description gáologique du département de l'Aisne. - 1843.

Le peu d'importance et l'éloignement des couches qui nous occupent dans ce 
département, nous défendent une critique détaillée; anssi nous contenteronsnous d'exposer quelques réllexions générales qui nous sont inspirées par l'examen des listes de fossiles de l'auteur.

M. d'Archiae, dont on ne peut soupeonner les tendances à donner des noms anglais aux couches du terrain jurassique en France, renonce absolument dans son travail à toute identification. Les travaux ultérieurs de M.I. Morris et Lycett démontrent que le niveau de Vinchinhampton y est très-reconnaissable par la présence d'un très-grand nombre de fossiles du Great-oolithe et l'absence presque complète des fossiles bajociens.

Nais entre ces deux niveaux se trouve une petite couche de peu d'épaisseur qu'il appelle oolithe inféricure ( $2^{c}$ assise du $2^{e}$ sous-groupe) et (qui contient,parmi les especes les plus communes, une grande partie de celles qui pullulent dans nos deux premières zones (Bathonien inférieur). Il est rrai que ees espèces sont en général celles qui forment ce que nous avons appelé la fuune nomade, e’est-àdire celles qui apparaissent ḑjà dans le bajocien pour remonter jusque dans le Callovien, mais qui ne se réunissent toutes que dans les deux premières zones et qui s'y reunissent d'une facon-eonstante (le earaclère le plus saillant de ce groupement des espèces est l'abondance de l'Ostrea remminaia et des Pleuromya).

Celte conche renferme : $1^{\circ}$ un on deux fossiles bajoeiens; 2' quelques espèces rares dans le bajocien, mais communes dans ces deux zones; $3^{\circ}$ quelques espèces caractérisant nos deux zones (1'Ammonites Parlitsoni manque); 4. un fossile de Minchinhampton.

Les caracteres de notre premicr groupe paraissant dominer dans ectle couche; l'oolithe inférieure de W. d'Areltiac parait représenter un Bathonien inféricur rudimentaire.

11 est vrai qu'une hésitation ne peut manquer de se manifester à la vue des listes de l'auteur où se trouvent mélangés des fossiles de toutes les couches jurassiques. Les idées théoriques de I. d'Arehiac ne parviennent pas à justifier des résultats en tout contraires aux lois connues de la palćontologie. Mais celte critique est trop en dehors de notre suję pour être traitée ici, nous nous contentons de dégager ee qui peut nous être utile : la constatation dans l'tisne rle nos couches à l'état rudimentaire, au double point de rue stratigraphique et paléontologique.

S III. MH. BUVIGLER ET SIUVAGE.

Statistique minéralogique et géologique du département des Ardennes. 1812.

Le département de l'Aisne était remarquable par l'état rudimentaire du Lias et l'absence du Bajocien; ici le Lias est bien développé, et le Bajocien alteint 
$120^{\mathrm{m}}$ de puissance. Il est même probable que les auteurs ont distrait quelques couches faisant partie du Bathonien pour les réunir au Bajocien, par ce motif, qu'elles étaient calcaires; cette confusion paraît évidente par la présence dans les banes de leur oolithe inférieure d'une colonie de Myaires qui ne descendent pas dans le Bajocien.

Notre groupe inférieur y est distinct au-dessus du Bajocien et assimilé au Fuller's Earth des Anglais, avec peu de fossiles, parmi lesquels pullule l'Ostrea acuminata; celte couche est appelée par les auteurs Marne inférieure; c'est notre Bathonien inférieur. Si on y réunit les couches à Pleuromyes et à Pholadomyes aux lumachelles oolihiques à grandes Huitres, ce groupe pourrait avoir $10^{\mathrm{m}}$ à $15^{\mathrm{m}}$ dans les Ardennes.

L'ammonites Parkinsoni n'est pas cité. Les couches supérieures commencent au-dessus de la Marne inférieure; leurs fossiles, en effet, indiquent nettement d'abord l'horizon de Minchinhampton, puis celui de la dalle nacrée, mélangés avec d'autres fossiles provenant de plus bas, notamment l'Ostrea acuminata; on y reconnait d'abord le Bathonien moyen, puis le Bathonien supérieur.

S IV. N. BUVIGNIER.

Statistique minéralogique du département de la Meuse. 1852.

A propos de ce travail, nous entrerons dans plus de détails que pour les travaux précédents, et notre crilique, au lieu de rouler sur des impressions générales, résultera de la discussion pied à pied, la notoriété de l'auteur et la proximité du terrain qu'il a étudié nous prescrivant une marche plus lente mais plus ulile.

Dès le début, l'anteur désespère de retrouver chacune des subdivisions anglaises; mais ses idées sur ce sujet n'étaient pas assez arrêtées pour empêcher l'introduction malheureuse du mot Bradfordien qui est du reste tombé dans l'oubli.

M. Buvignier divise l'étage inféricur du terrain jurassique en trois groupes:

$1^{\circ}$ Oolithe inférieure; $2^{\circ}$ Marnes du Bradford-clay; $3^{\circ}$ Calcaires gris oolithiques. Examinons successivement chacun de ces trois groupes.

$1^{\circ}$ Oolithe inféricure $\left(130^{\mathrm{m}}\right)$.

3 Sous-groupes.

Sous-groupe inférieur. - Calcaires terreux jaunâtres, peu consistants, renfermant une grande quantité de Polypiers (1). L'auteur pense que cette couche est le prolongement du calcaire à Polypiers de la Moselle; la liste des fossiles qu'il donne indique au reste une faune bajocienne.

(1) - Notons en frassant un fail commun avec M. d'Archiac (Géologie de l'disne) et que M. Buri. gnier laisse vir aussi dans la Giologie des Ardennes: la plupart des polypiers, sont des espèces oxfordiennes, quelquelois coralliennes; il en est de même pour un cerlain nombre de Peignes, de Limes, etc., qui accompagnent toujours les colonies de polypiers. 
Sous-groupe moyen. - Grande variété de calcaires terreux compactes, oolithiques, sans ordre constant de superposition; marnes sableuses grises au sommet. La liste des fossiles est très-embrouillée; la difficulté de suivre les couches à cause des éboulis peut seule expliquer les anomalies paléontologiques qu'on y rencontre. En effet, dans les Nyaires, nous trouvons des Pholadomyes Bajociennes, des Pholadomyes communes au Bajocien et au Bathonien, enfin, des Pholadomyes propres au Bathonien inférieur; nous y trouvons aussi toute la tribu des Pleuromyes caractéristiques de notre $2^{\mathrm{e}}$ zone.

S'il est vrai qu'une faune Bajocienne y est incontestable (Ammonites Trucllei, A. Braidkenridgii, A. Brongniarti); il est vrai aussi que beaucoup de fossiles caractéristiques de deux de nos zones s'y rencontrent.

La conclusion la plus probable, e'est que la difficulté de recueillir des fossiles en place, a masqué à l'auteur la séparation des deux étages et lui a fait mélanger les fossiles de couches différentes. Aussi ne pouvons nous nous empêcher de reconnaitre nos marnes de Longwy dans le sommet de ec sous-groupe.

Notons en passant la richesse de notre faune nomade, représentée ici par Isocardia gibbosu, Cucullau oblonga, C. clongata, Trigonia costata, Gervillia lanceolata, Avicula tegulita, A. Bramburiensis, Lima proboscidea, Ostrea crenutu, $O$. sandelina, Terebrnula perovalis, $T$. ornithocephala, T. bullata.

Sous-groupe supéricur. - Caleaires terreux à grains très-fins, jaunes, contenant des oolithes, des lamelles splathiques et des fragments de coquilles triturées.

L'auteur affirme avoir suivi ees calcaires dans la Moselle et la Meurthe. Cette remarque et la description de la roche conviennent parfaitement bien au calcaire de Jaumont et à lui seul.

En résumé, nous pouvons conclure que l'auteur n'a pas vu qu'une séparation était nécessaire à effectuer vers le sommet de son groupe. 11 est certain que, s'il avait pu mieux observer ces marnes grises, il aurait fini par y trouver l'Ostrea acuminata, qui est rare dans les marnes de Longwy, quand elles sont sableuses, ou mème dans le calcaire Iumachelle, où cependant elle se trouve plus ou moins reconnaissable à ses débris.

Le synchronisme pourra done se faire de la manière suivante :

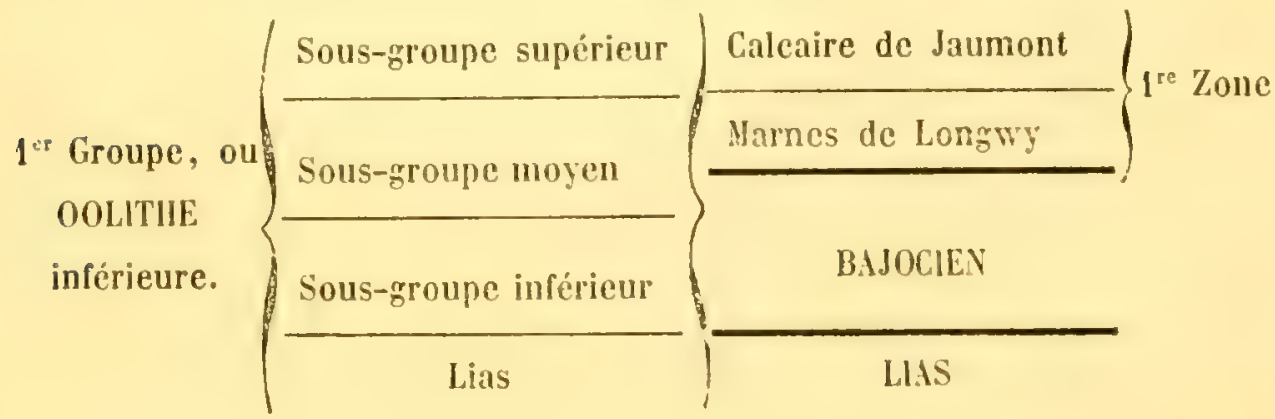


" $2^{\text {me }}$ groupe. Marnes du Bradford-Clay $\left(80^{\mathrm{m}}\right)$. Aliernance de couches marneuses et calcaires, de nature variable, se succédant sans régularité. „

"Les calcaires sont généralement marneux et en banes minces; quelquefois ils sont oolithiques et en banes épais; quelques banes oolithiques renferment le Clypeils patellı. Les argailes sont grisâtres ou bleuatres; elles contiennent quelquefois des oolithes ferugineuses; on observe principalement des oolithes dans des bancs renfermant en abondance des Anabacia et des Terebratula."

Cette description est tout à fait celle de nos marnes de Gravelotte avee les calcaires qu'elles comprennent ou cenx qui leur sont superposés.

Outre la faune nomade, ces argiles bradfordiennes contiennent des fossiles tels rue l'Ammoniles Purkinsoni, Clypers patella, etc., caractéristiques de la $2^{\mathrm{e}}$ zone.

" $3^{\mathrm{e}}$ grroupe. Calcaires gris oolithiques $\left(60^{\mathrm{m}}\right)$. Bancs calcaires blanchâtres, gris ou bleuâtres, généralement oolithiques, quelquefois terreux, ces deux variétés passant de l'une à l'autre. ”

On peut reconnaître à celte description l'ensemble des ealeaires du Jarnisy et des calcaires oolithiques blanes, sans fossiles, qui les supportent.

D'après la liste de l'auteur, les fossiles de ces calcaires appartiennent tous au groupe précédent, et aucun d'eux ne caractérise le Bathonien supéricur.

Ces synchronismes ne font aucun doute et résultent assez rigoureuscment des listes de fossiles que nous ne donnons pas pour ne pas allonger la discussion. (On peut voir que nos couches, dans la Mcuse, sont bien plus épaisses que dans la Moselle).

H. Buvignier finit là l'étage inférieur du terrain jurassique et eommence l'0xfordien aussitôt.

L'étude des environs de Conflans nous a démontré que M. Buvignier fait descendre l'oxfordien beaucoup trop bas. La faune change bien au-dessus des " calcaires gris oolithiques », mais pas assez pour rattacher à l'0xfordien des couches qui non-seulement méritent leur indépendance de ce côté, mais qui ont même beaucoup plus d'affinités avec les " calcaires grris oolithiques »

M. Buvignier, décrivant les argiles inférieurs de l'étage moyen du terrain jurassique, donne une liste de fossiles intéressante à consulter.

Cette liste présente des espèces pouvant être divisées de la manière suivante:

$1^{\circ}$ Espèces oxfordiennes, 10 ; or , sur ces dix espèces, quatre sont marquées d'un point de doute, une (Pecten anopleurus) espèce de M. Buvignier, n'a pas été retrouvée ailleurs et est reproduite telle quelle dans le Prodrome de d'Orbigny. Il reste done cinq espèces de bon aloi : Gryphaa dilatata, Trigonia elongata, Ammoniles Duncani, A. modiolaris, A. dentatus. C'est tout ce qui reste d'une longue liste comprenant plus de 50 espèces; et encore ces cinq espèces oxfordiennes descendent-elles jusqu'ù la limite inférieure des a calcaires gris oolithiques $\infty$ ? 
$2^{\circ}$ Fspèces inférieures à l'Oxfordien: 7 , don! 2 bryozoaires de Ranrille; Cerioporr dumetosa, Cricopore Tessoni; puis ä especes se trourant daus le Bathonicis: Nucula lacryma, Pecten fibrosus, Avicula aigitata, Ostrea acuminata, O. Knorrii.

En faut-il conclure qu'il y a mélinge naturel de ces deux catégories de fossiles?

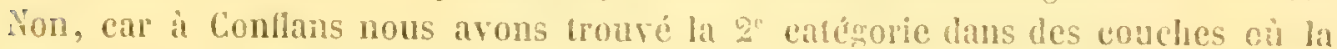
première fasair entierement defaut. Quant à la cause de l'errenr, la volei: certaines couches de la $3^{2}$ zone ressemblent à celles tie la $2^{\circ}$ zone et a celles ou se trouvent la Gryphcea dilalala et l'Ammoniles Duneuri; mais la présenee de l'Ammonites quercinus et de la Terebratula layenalis distingue cette zene de l'Oxforticn et de la 2" zone, malgré une identité presune complète dans la roche.

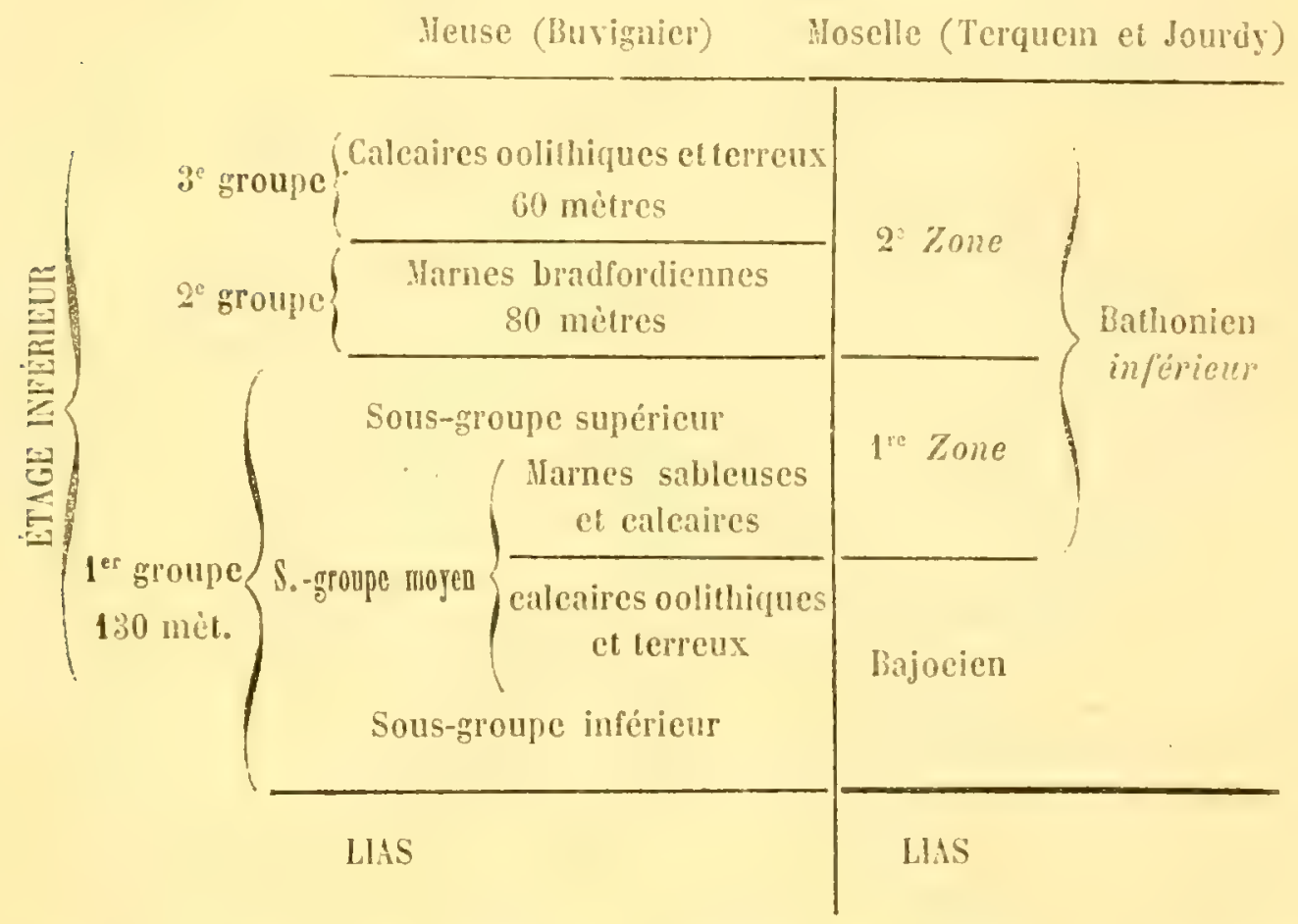

On voit que non-seulement nous ne partageons pas les idées de M. Buvignier sur la délimination de l'oxfordien, mais que nous arêtons différemment le bajocien; de plus, tout en décrivant les mêmes couches, nous les groupons d'une maniere bien diflérente. Quoiqu'il en soit, nous arons vu qu'on peut suivre le Bathonien non-seulement dans la Meuse où la pétrographie est à peu près la même que dans la Moselle, mais encore plus à l'ouest, oủ les indications tirées des fossiles en font reconnaitre la présence. 


\section{V. SYNCHRONISHE GÉNÉRAL.}

Cette étude serait sans utilité si on se contentait de montrer comment les observations précédentes manquent de lien et de rigueur; mais on peut, avec une certaine certitude, tirer des fuils précédents un certain nombre de conclusions générales qui les relient ensemble et qui peuvent servir de base au premier travail de synchronisme.

Dans l'Aisne et les Ardennes le fait stratigraphique dominant est le grand développement des couches oolithiques, renfermant la faune de Minchinampton qu'on peut considérer comme représentant le Bathonien moyen, tandis que la partie inférieure est ou rudimentaire (Aisne) ou bien très-peu développée (Ardennes).

Dans la Moselle et la Heuse, le Bathonien inférieur est richenent développé; le Bathonien moyen manque.

Cet inégal développement des couches des différentes parties du Bathonien n'est pas le fail le plus frappant qui ressorte de cette étude.

Dans l'Aisne et les Ardennes, les couches inférieures du Bathonien sont terreuses, tandis que les couches supéricures sont calcaires et généralement trèsoolithiques (oolithe de Rumigny).

Dans la Moselle et la Heuse, l'inverse se produit, et, tant que la Paléontologie permet d'affirmer qu'on reste dans les limites des deux premières zones, les ealeaires presque toujours très-oolithiques dominent, ou sur une grande épaisscur, ou en s'intercalant par de nombreux banes, quand les couches sont marneuses. Au contraire, l'apparition de l'Ammonites quercinus assiste à un développement de marnes très-argileuses avee quelques couches de calcaires, se délitant facilement et imprémgés d'argile.

Mais ces différences plus ou moins abstraites entre les deux faciès peuvent s'exprimer d'une façon plus générale, en interprétant les données fournies par les fossiles.

Dans les Ardennes et l'Aisne, la faune du Bathonien inférieur est représentée par des fossiles qui montent ou descendent, par ce que nous avons appelé la faune nomade.

Dans la Moselle et la lleuse, non-seulement la faune nomade y est représentée par un très-grand nombre d'espèces, mais, de plus, la faune caractéristique s'y montre avec une grande exubérance. Ces résultats étaient faciles à prévoir, étant donnée la différence de puissance des deux faciès; car, à priori, il y a plus de raison pour que le développement de la faune dans une zone soit plus complet quand cette zone est représentée par des dépôts d'une grande épaisseur, de préférence au cas où cette zone ne se compose que d'une couche de peu d'importance. 
Dans l'disne et les Ardennes, autant le Bathonien inférieur est paure en fossiles caractéristiques, autant les couches qui le surmontent conticnnent de fossiles spécianx en grande quantité (couches à Rhynchonella decorata). La faune d'Eparey, d'tubenton et de Rumigny, contient une colonie de rastéropodes des plus remarquables, est abondante en polypiers, en brachiopodes, avec peu d'acéphales et sans céphalopodes, ce qui démontre une station coralligène.

Dans la ileuse et dans la Hoselie, dès qu'on sort du Bathonien inférieur, la richesse de la faune diminue de plus en plus avee la hauteur. La zone à Ammonites quercinus et surtout la zone supérieure sont bien moins riches en fossiles caractéristiques; cependant la faune u'y est pas atrophiéc, surtout à la base oủ elle aequiert son plus grand développement; ses fossiles autres que les brachiopories (et ceux-ci abondent dans bien d'autres couches), sont en général de petites Huîtres, des Gresslyes, des Peignes, des Nucules, ele., témoignant une station vasog̀̀ne. (1)

Ainsi d'une part (Ardennes) faune peu stable et peu riche pour la base du Bathonien, faune des plus abondantes et des plus caractéristiques pour les couches placées au-dessus. Ailleurs (Moselle), faune riche en toute sorte d'espèces pour la base du Bathonien, fame pauvre et mal renouvelée pour le sommet.

On voit que ce fait paléontologique est bien dans le même sens que celui de la puissance relative des couches.

Les conditions ont donc été bien différentes après la fin du Bathonien inférieur dans les Ardennes d'une part et la Moselle de l'autre; nous avons déjà vu que dans les Ardennes la zone à Ammonites quercinus fait défaut, tandis que dans la Moselle ce sont les couches a Rhynchonella decorata qui manquent; or, dans les pays où la série est complète, les couches des Ardennes sont placées au-dessous des couches de la Moselle, de telle sorte qu'on ne puisse pas les supposer contemporaines.

La différence des faciès entre l'Aisne et la Meuse se manifeste d'une façon encore plus atcentuée dans les roches et les fossiles, après un premier renouvellement de la faune bathonienne; ce qui fait supposer que celte différence

(1) On peut faire ici un rapprocbement curieux sur les conséquences que ces différeals faciès ont produites dans l'Aisne el la Meuse.

Dans lasne, M. ditrchiac a identifé un grand nombre de ces espéces de polypiers, ainsi que des genres qui habilent autour (Nérinées, Purpurines, Peignes, Limes Pholades, etc), avec des espéces de Goldfuss, cilées comme appartenant à loxforilien et au Corallien, terrains dans lesquels les stations coralligènes acquièrent de grands développements.

Dans la Mcuse, M. Buvignier prélend avoir trouvé dans notre zone à Ammonites quercinus (la base de son Oxiordien), les trois especes suivanles: Thecocyathus mactra, Tintinabulum et Nucula Uammeri, fossiles caractéristiques du lias supérieur, qui babitent les stalions los plus vaseuses de celle formation.

Il est incontestable que de mavaises déterminations ont seules pu produire ces deux résultats; mais aussi il est intéressant de constater que les faunes vasogènes ou coralligrenes de deux terrains différents renferment des especes assez. voisines pour fromper des paléonlologistes habiles; ce qui montre que le renouvellement des faunes a travers les élages est loin le se idire dune facon aussi brusque quion l'admet trop souvent. 
de faciès ne tient pas à une cause passagère; aussi pensons-nous que ces différences, dont la recherche est une partic intéressante de ce travail de synchronisme, proviennent de causes inhérentes à l'assiette mème des terrails.

Si on consulte la Carte géologique de la France, on voit que la région où le Bathonien infériem est atrophié pendant que le bathonien moyen est très-riche et que le Bathonien supérieur manque, est celle qui borde le pied des Ardennes; le faciès particulier à celte région sera le faciès Aldennais.

On reconnait aussi que la Hoselle et la Meuse se relient à la contrée géologique (la Lorraine) qui s'étale au pied occidental des Vos?ces. Dans cette contréc, le Bathonien inférieur est toujours richement doué de ses fossiles earactéristiques, tandis que le Bathonien moyen manque et que le Bathonien supérieur est relativement paure en fossiles; pour cette raison, nous appellerons ce faciès: le faciès Vosgien (1).

L'Arlenne plongeant à pic, par ses montagnes schisteuses, dans la mer jurassique devait plus particulièrement donner des falaises où les colonies de polypiers, accompagnées de leurs hôtes habituels, devaient trouver un facile développement et dépeupler rapidement la station des espèces établies lors de l'étage précédent, dont les unes ont disparu, les autres moins scusibles au changement passèrent dans le sous-étage suivant, en formant une partic de la fanne nomade.

Si, des bords de l'Misne on se dirige vers ceux de la Meuse, la roche primaire se masque de plus en plus, recourerte par les dépôts "lu Lias et son action immédiale sm le faune s’atténue de plus en plus en s'avanẹnt vers la Chiers. II y a là (d'antres les listes de M. Buvignier et Sauvage) un moment de transition où la série bahonienne est complète.

Les Yosges, au contraire, présentant à la mer la séric des plans inclinés, formés par des terrains émergés sucessivement et aree lenteur, devaient donner de nombreuses stations vaseuses, où les Myaires pouvaient se développer facilement. Les dépôts ne sont réellement calcaires et oolithiques qu'au voisinage du Bajocien, quand le régime ressemblait encore à celui de l'époque où les polypiers abondaient avec les calcaires compactes; mais, dès qu'on s'éloigne de cette époque, les dépôts vaseux se déreloppent avec une abondance qui rappelle les argiles du Lias et de l'Oxfordien. La transition entre ces deux régimes se remarque dans les calcaires du Jarnisy ( $2^{c}$ zone). La zone supérieure assiste au règne des dépôts vaseux, contenant par conséçuent peu de fossiles qui caractérisent une zone où pullulent généralement les gros polypiers et les bryozoaires. Malgré les différences de dépôts et de faunes, le faciès apdennais et le faciès vosyien renferment également d'immenses quantités de Térebratules et de Rhynchonelles ;

(1) Dè difrérences de faciès se constalent aussi pour le Lias dans les deux contrées des Ardennes et des Vosges. (Voyez Terquem et Pistle, Mémoire sư le Lias infeirieur de l'Est de la France) 
la facilité avec laquelle ces brachyopodes s'acclimataient à des conditions si diverses explique bien la difficulté que les palćontologistes éprourent dans les déterminations scientifiques.

Un faciès intermédiaire en quelque sorte entre les deux est celui qui se rencontre dans le Jura.

Là le voisinage des Vosges et la continuité des dépôts avee ceux de la Lorraine expliquent bien l'analogie paléontologique que le Ballonien inférieur présente à la fois dans la Hoselle, la Haute-Saóne et le Jura; la faune caracléristique y est nettement déreloppée sur une grande étendne. D'un autre côté, au Sud, les Vosges, plongeant dans la mer à peu près comme les Ardennes, permirent ultérieurement un développement considérable de calcaires; e'est pourquoi plus on s'avance au Sud-Est, e'est-à-dire plus on s'éloigne du litloral, plus la base du Bathonien s'évanouit et tend au type ardennais. Comme exemple de ces deux faciès dans le Jura, citons d'une part Vesoul et Champlitte où le type Vosgien est bien développé, de l'autre le Jura Neuchâtelois où la zone est complètement annulée; plus loin, dans le Jura Central et le Jura Oriental, le groupement des rossiles indigue une région différente.

Comme points intermédiaires, citons Dôle et Salins, oì l’Ammoniles Parkinsoni se présente arce une grande fixité de forme et d'habitation, et où les trois parties du Bathonien sont tellement bien caractérisées par les roches et les fossiles que celte partic du Jura occidental est vraiment le type de ces terrains, tandis que la contrée des Ardennes et la Lorraine offrent chacune une anomalie inexplicable si on ne les compare aux localités types.

\section{\&. N. PIETTE.}

Note sur le gîle des Clapes (Bulletin de la Société géologique : 1857).

M. Pielle s'est proposé, dans une petite note, de définir exactement la position stratigraphique d'un gite déjà très-connu des collectionneurs et auquel nous. devons nos plus beaux fossiles.

Il a domé une coupe de \$ont-Saint-Wartin à Longuyon, dans laquelle il attribue aux couches à Ostrea acuminata (avec leurs calcaires) une épaisseur exagérée; une simple course sur le plateau de Longwy suffi pour recomaitre l'erreur.

II. Jacquot avait protesté contre cette coupe et donnait comme raison du malentendu la présence de failles que l'auteur n'avait pas vues. I. Dargniès a vérifié ce fait; en parcourant le plateau, il a vu qu'on rencontrait souvent les deux mêmes couches, grâce à des ressauts nombreux (oolithes de Jaumont et marnes de Longwy).

I. Piette, ne tenant pas compte de cette particularité, additionnait toujours

SOC. GËOL. - 2 SÉRE T。 IX, - MEM。 $\mathrm{X}^{\prime \prime} 1$. 
les banes calcaires aux banes marneux chaque fois qu'il passait des marnes aux calcaires ou inversement, ec qui explique à la fois et l'épaisseur extraordinairc (plus de 50 mètres) qu'il donne à la $1^{r e}$ zone et à l'ensemble formé par la première zone et les banes de la deuxième ainsi que les alternances réitérées des roches.

Cependant il indique assez nettement ce fait, fque les marnes des glacis de Longivy (Marnes de Longwy) sont surmontées par des calcaires oolithiques (Calcaires de Jaumont) sur Iesquels repose le gite des Clapes (Harnes de Gravelotte).

Tout en reconnaissant la position particulière des Clapes, l'auteur a méconnu la position véritable de cet horizon dans le système général des roches qui surmontent le Bajocien. Il assimile l'horizon des Clapes au Fuller's-Earth, les marnes de Gravelotte à la grande oolithe et méme mieux les caleaires de Failly au Cornbrash.

Toutes ces hypothèses sont gratuiles el peut-être sont-elles venues à l'esprit de l'auteur à la vue de données paléontologiques incomplètes; mais la stratigraphie d'une part et la paléontologic de l'autre les condamnent entièrement.

Il esi vrai que M. Piette n'attache pas grande importance aux synonymies anglaises; nais, en se débarrassant de cette cause d'erreurs, il en introduit une autre par l'adoption du mot Bathonien qui ne pouvait alors être d'aucune utilité parce qu'il était mal défini.

S II. M. O. TERQUEN.

Mémoires sur les Foraminifères du Système oolithique. - 1867.

Dans unc introduction à son étude paléontologique, M. Terquem assimile au Fuller's-Earth les deux premières zones et en indique les limites probables; c'est la premic̀re tentative ayant pour but de reculer à l'Ouest les limites de ce terrain. On pensait auparavant que le Fuller's-Earth finissait aux collines tominant la rive gauche de la Moselle; dans ce mémoine, cette limite est reculée jusqu’’à 23 kilomètres au delà de Gravelotte, e'est-à-dire dans les environs de Conflans.

M. Terquem, dans ses recherches mieroseopiques, avait été conduit à recueillir une magnifique collection de fossiles; s'appuyant sur des données paléontologiques beaucoup plus complètes que celles de H. Pieste, il a reconnu la véritable place de la faune de Gravelotte et affirmé l'absence, sur le plateau que nous avons décrit, des fossiles caractéristiques de la grande oolithe, ce qui, à cettc époque, était déjà une hérésie dangereuse. Du reste, ces indications ne s'étendent qu'aux deux premières zones, les seules qui pussent intéresser, par leurs couches marneuses, des recherches ayant pour but des travaux microscopiques. 


\section{§ III. M. JACQUOT. \\ Description géologique et minéralogique du département de la Moselle. - 1868.}

Le livre de $\mathrm{N}$. Jacquot est l'explication d'une earte géologique coloriće et dressée par feu M. Reverchon. Le livre et la carte sont, du reste, assez peu d'accord, surtout pour le terrain qui nous occupe.

La carte est bien inféricure au live; les divisions stratigraphiques sont me̊me bien en retard des idées généralement reçes en géologie; pour en donner une idée, il suffit de citer les divisions auxquelles sont affectées des teintes : .

$1^{\circ}$ Cornbrash, Forest-marble. - Calcaires à oolithes difio:mes (partic de la 2 zone;

$2^{\circ}$ Bradford-clay. - Grande oolithe; oolithe jaune. - Fuller's-Earth pierreux (reste de la $2^{\mathrm{e}}$ zone et partic de la $\mathrm{A}^{\mathrm{rc}}$ );

$3^{\circ}$ Fuller's-learth marneux (reste de la $1^{\text {re }}$ zone).

Il est inutile de discuter cette classification, lc choix des teintes correspondant aux divisions adoptées ainsi que leur emploi. Nous avons fait justice, dans une note lue à la Société géologique le 21 juin 1869, de ces idées en retard de trente années, bien qu'elles aient été publiées nouvellement.

Les idées de M. Reverchon ont aussi servi à M. Élie de Beaumont, qui probablement n'a pas pu éludier ces terrains à cause de la difficulté des transports sur le plateau, et qui a utilisé les notes manuserites de cet ingénieur pour sa carte géologique.

II. Jacquot a publié un iexte sur cetie partie de ses éludes en collaboration arec N. Barré; il a donné une description exacte des différentes roches décrites cidessus. L'auteur admet bien le Bathonien; seulement il l'arrete à la $2^{\mathrm{e}}$ zone; pour lui, ies conches supéricures sont de l'oxfordien, étage qui rémnernit alors de Conflans à Verdun; malgré les restrictions de son collaborateur M. Barré, dont les notes ont servi à la deseription géologique du pays à l'ouest de la Hoselle, H. Jaequot trouve une différence très-grande cntre la $2^{e}$ et la $3^{e}$ zone, où il place la limite de l'oxfordien. Cependant la différence pétrographịue entre la base de l'une et le sommet de l'autre est complètement nulle; quant au caractère paléontologique, comment le définil à une époque oủ nos fossiles n'étaient pas encore classés?

Enfin M. Jaequot a vu dans les formes topographiques de ces deux zones des allures assez différentes pour supposer qu'il y avait là des dépôts entre lesquels aucun rapprochement n'était possible. La première assise de la $3^{\mathrm{c}}$ zone forme à Conflans quelques buttes isolées, dont la base, un peu calcaire, se délache nettement du sommet de la $2^{\text {e }}$ zone formée par des argiles étalées en pente beaucoup 
plus douces et dessinant une sorte de ceinture humide où les prés sont abondants; or, ce fait visible à Conflans où la base de la $3^{\circ}$ zone est un peu calcaire, disp̣araît complètement dans une foule de localités où des argiles assez épaisses s'intercalent dans les banes calcaires; ce caractère topographique, vrai en tant que fait particulier, devient faux quand il est considéré comme fait général.

L'Oxfordien ne se trouve nulle part dans le département de la Moselle; pour rencontrer cet étage, il faut aller dans la Meuse. Malgré cette erreur, qui est grave, M. Jacquot groupe à peu près comme nous les couches au-dessous de l'oxfordien; cependant, en entrant dans les détails, les divergences sont encorc assez considérables.

L'oolithe inférieure (correspondant au Lower Oolite) est divisée en trois groupes : le premier comprend l'assise inférieure ou calcaire ferrugineux et l'assise supéricure ou calcaire à polypiers; le second (notre $1^{\text {re }}$ zone) comprend l'assise inférieure (marnes de Longwy) et l'assise supéricure (calcaire de Jaumont); la troisième (notre $2^{\mathrm{e}}$ zone) comprend l'assise inférieure (marnes de Gravelotte) et l'assise supérieure (série des calcaires de la $2^{\mathrm{e}}$ zone) (1).

Le premier groupe est le Bajocien

Le second et le troisième sont nos deux premières zones, mais avec une nuance qu'il est impossible de laisser passer. Pour nous, ees deux zones ont tellement de points communs (quant à la faune) que nous avons dù les réunir pour former une division d'importance égale à une des divisions du Bajocien. Pour M. Jacquot, au contraire, le troisième groupe $\left(2^{\circ}\right.$ zone) est a aussi distinct par sa pétroğraphie que par sa faune des deux précédents (page 260). „0r, e'est admettre que ces trois groupes étant séparés par des différences égales, chacun des deux derniers est équivalent au premier, ce qui est absolument contraire aux faits paléontologiques. Car les deux zones renferment à elles deux plus, de 350 espèces, flont 47 seulement sont bajociennes, et cependant la faune du bajocien est extrênement riche et renferme une faune bien aussi nombreuse que celle du groupe des deux zones; c'est à peine s'il y a le 1/7 d'espèces communes du Bajocien au Bathonien inférieur. La faune de chacune de ces deux zoncs est reliée à celle de l'auire par des espèces plus nombreuses que les espèces spéciales à la $1^{r e} z o n e$, el qui forment le quart des espèces spéciales à la $2^{\mathrm{c}}$ zone (qui possède un grisement exceptionnel); ce qui démontre bien qu'après le premier groupe eut lieu un renouvellement de la faune incomparablement plus considérable qu'entre les deux zones.

Enfin, pour séparer plus nettement le deuxième groupe du troisième, M. Jacquot donne cette raison que les marnes de Gravelotte assistent à l'apparition de fossiles nouveaux, notamment le Clypens patella (il aurait pu ajouter l'Ammoniles Parkinsoni non moins caractéristique). De là une différence de 
même ordre que celle qui résulte de l'apparition de l'Ostrea acuminala dans le second groupe, et qui justifie l'établissement de cette division. Or, ces deux apparitions ne sont pas du mème ordre, car la présence de l'ostrea acuminata, tant que la faune ne subit pas de variations sceondaires et jusqu'à ce que la faune se modifie plus profondément, est un caractère d'étage, tandis que la présence du Clypeus patella, qui se constate tant que la faune ne subit aucune modification et qui cesse des que cette faune change quelque peu, est un caractère de zone.

Enfin, M. Jacquot, dans une note, admet que le caleaire de Jaumont commence le niveau du Great-0olite. Y a-t-il cependant une variation de faune?

Nous n'insisterons pas davantage sur ces erreurs qui défigurent complètement l'idée qu'on peut se faire du Bathonien dans la Moselle; nous les avons seulement montrées pour faire voir combien il était nécessaire de prendre une nouvelle direction dans la description de ces couches, qui ont une grande importance par leur étendue et leurs caractères. C'est dans ce but que nous avons entrepris ce travail préliminaire de l'étude palćontologique, en envisageant la question au point de vue multiple des roches, de la faune et de la relation de notre terrain avec ceux du même horizon.

Dans le cours de cette introduction, nous arons eu plusieurs fois l'occasion de citer M.I. Barré et Dargniès, qui nous ont communiqué, arec beaucoup de bienveillance, les résultats de leurs explorations. Nous devons de mème témoigner notre reconnaissance aux personnes amies des seiences, M. Deschange, de Longuyon, II. de Lambertye, de Cons-la-Granville, et II. le docteur Colliez, de Longwy, qui ont bien vonlu nous communi iuer les échantillons les plus rares de leurs collections et qui ont ainsi contribué à compléter nos séries paléontologiques.

Nous avons enfin à témoigner notre gratitude à II. Polteau (préparateur au Aluséum, section de Conchyliologie), qui, avec une complaisance extrème, a mis à notre disposition sa riche collection de spicules et contribué de la sorte au classement si difficile des espices fossiles.

La localité si remarquable des Clapes a été, dans le temps, découverte par I. Levavasseur, qui, aree un désintéressement très grand ct fort rare, nous a fait don de presque tonte la série que nous publions. 


\title{
DEUXIEME PARTIE.
}

\section{PALÉONTOLOGIE.}

\author{
PREMIÈRE SECTION.
}

\section{INTRODUCTION.}

Dans le département de la Moselle, la faune du Bathonien se présente avec une abondance remarquable de fossiles. Si leur étude et leur classification ne sont pas dépourvues de difficultés, leur abondance même nous permet d'en dégager une inconnue, c'est-à-dire, nous autorise à signaler à l'attention des géologues celles des espèces qui, à nos yeux, sont appelées à remplir le rôle important de caractéristiques du terrain.

Comme nous l'ayons exposé dans la partie stratigraphique, les fossiles peuvent être divisés en plusieurs calégories : $1^{\circ}$ ceux qui commencent à se produire dans le Bajocien inférieur et ne dépassent pas la première zone du Bathonien: $2^{\circ}$ ceux qui commencent de même et se continuent au delà, jusque daus l'0xfordien et même le Corallien; $3^{\circ}$ ceux qui se présentent dans le Bajocien supérieur (le Calcaire à polypiers ou subcompacte) s’arrêtent dans le Bathonien inférieur;

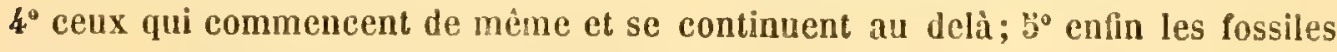
qui sont propres à chacune des zones.

Ce n'est pas que nous voulions considérer toutes les espèces nouvelles comme caractéristiques; nous croyons au contraire que quelques-unes sont propres à certaines localités, quui ont servi de rivages; d'autres appartiennent à des mers 
plus ou moins profondes et par conséquent à certaines stations qu'clles n'ont pas dépassées; dans ce cas leur présence devient un guide certain pour la classifieation des zones dans d'autres provinces, où l'on pourra ainsi reconnaitre leur succession normale ou leur atrophie plus ou moins profonde.

La faune du Bathonien étant jusqu’à ce juur incomplètement connue, la délimitation du terain n'a pu ètre exactement tracée el les autcurs en ont fait, en général, une mention très-succincte ou même fautive.

D'Orbigny (Straligraphie paléontologique, t. II, fase. 2, p. 478 ct 492) a rapporté le ierrain qu'on appelait Fuller's-Ekr/h au Bajucien et les marnes à 0 . acuminala qu'il a supposé former une autre assise au Bathonicn; de là, dans le Prolome, un mélange inextricable de fossiles appartenant a des horizons très-différents; d'Orbigny a ainsi consacré des erreurs dépendantes en partie des envois qui lui avaient été faits avec des indications plus ou moins irrégulières.

Celte connaissance incomplète de la faune caractéristique des diverses assises de l'Oolithe inférieure a cncore porté d'Orbigny, considérant Bayeux comme une lccalité typique, à imposer le nom de Bajocien au premier groupe oolithique.

Nous signalerons en particulicr deux Ammonites qui se truuvent à Bayeux, le subfurcatus (niortensis, d'Orb.) et le Parkinsoni, qui se montrent dans nos parages, constamment séparées par environ 40 mètres de roche; elles ne se rencontrent jamais avec le Sowerbyi, également de Bayeux, et dont elles se trouvent éloignées par un dépôl d'une grande épaisseur.

Quenstedt, dans son Jura, a consacré les planches $\$ 2$ et 33 aux fossiles du Bathonien inférieur qu'il range dans son assise d’; dans le texte, il en mentionne les principaux, qui répondent exactement à ceux que nous avons rassemblés.

Dans le catalogue paléontologique du liusée de Londres, nous yoyons l'assise à 0 . acuminata ne contenir qu'un ensemble de 38 espèces (p. 193 et 225) qui toutes se retrouvent dans nos parages; nous devons faire observer, d'une part, qu'on y voit figurer 4 Panopées (probablement des Pleuromyes) et une Anatine (Arcomye); d'une autre part, il est à regretter que, dans ce volumineux et important travail, les noms des espèces ne soient pas suivis de ceux des auteurs; cette indication est indispensable pour les recherches et le elassement raisonné des fossiles.

La superficie du sol occupée par cette assise inféricure présentant une grande élendue a pu offrir à l'observation une grande variélé dans la nature des dépòts, calcaires ou marneux; de la sorte chaque localité a apporté un tribut particulier à l'ensemble de la faune. Toutefois il convient de faire remarquer que certaines localités ont subi des modifications depuis l'époque où nous avons exercé nos recherches: les unes ont été complètement exploitées ct ont disparu (Amanviller, d̀ la sortie de la vallée de Montvaux); les autres ont élé nivelées ou sont devenues inabordables (Fontoy, niveau de la voie, talus de la gare). D'autres encore ne présentent de fossiles que lorsqu'on y a pratiqué des fouilles ou que la charrue en 
a renouvelé la surface (Longwy (glacis), les Clapes, Gravelotte, Gorze (cimetière).

Pour la zone inférieure nous avons à citer en particulier le plateau de Longwy et les gracis de la ville; à la vérité, la localité s’épuise, la surface n’étant pas souvent remuée, mais les fossiles caractéristiques y sont tellement abondants, que de longtemps ils ne feront défaut. Dans la zone moyenne, $1^{\circ}$ les Clapes ont donné une faune extraordinairement riche et en tout point comparable à une faune tertiaire pour la rare conservation des fossiles (1); $2^{*}$ Fontoy, où les grands fossiles sont assez rares, mais où les fossiles microscopiques el les foraminifères en particulier foisonnent exceptionellement; leur abondance en espèces et en variétés est telle, qu'elle a obligé d'établir des monographies de genres, dont deux, celles des Marginulines et des Cristellaires sont publiées; $3^{\circ}$ Gravelotte, fréquemment travaillé pour la culture, présente une localité inépuisable de nombreux fossiles; $4^{\circ}$ quelques localités, telles que Gorze (cimetière), Thiaucourt (les vignobles), peuvent toujours être visitées avec quelques chances d'y trouver les fossiles que nous indiquons. La zone supérieure comprend les limiles extrêmes du département de la Moselle qui se confondent avee celles de la Meuse et dont N. Colliez a trouré un îlot entre Cutry et Chenières, près de Cons-la-Grandville; cette zone fait un angle rentrant près de Hloineville, entre Auboué et Conflans; en effet sur les plateaux d'Auboué, on trouve la réunion des mèmes fossiles qu'à Gorze, Jarny, etc., mais de transport el à l'état roulé.

L'élude stratigraphique des fossiles, exactement élablic et délimitée, a pu c̀tre exposée dans un tableau général, qui montre la station première de chaque espèce, ainsi que ses pérégrinations et ses passages successifs dans les diverses couches et assises.

Ainsi que nous l'avons mentionné plus haul, nous ne saurions suivre les indiealions de d'Orbigny pour la classification des espèces; nous nous contenterons de les mentionner à la suite de nos descriptions; nous nous laisserons guider par les collections que nous arons à notre disposition et qui toutes appartiennent à notre département; ayant ainsi sous les yeux toutes les séries de fossiles avec les

(1). Les fossiles se troureut continés dans un champ qui ne comprend que 4 ou 5 rait's de cullure, ayant environ 100 metres de largeur sur 2ä) de longeur et ne se distinguant pas par leur constilution pétrographique des lerres avoisinantes

Bien que les indications fournies par l'cascmble des fossiles fussent concluantes pour la classificalion da la localilé, nous avons cependant voulu connalire la nature du sous-sol el nous avous pu, gràce au bienveillant concours de M. Deschange, y faire pratiquer des fouilles; le sol arable, formé de marnes, d'un jaune brun, lérèrement calcareuses et ferrugineuses, a une épaisseur variable de 40 à 60 centimètres; au dessous se présente uniformément une rocbe jaune, calcaréo-marneuse, injectć d'bydroxyde de fer, qui, dans ses couches superieures renferme encore quelques fossiles qui se trouvent dans les marnes; bientôt, et en même temps que l'hydrosyde, les fossiles disparaissent et on obtient un calcaire a très yeu près semblablo à celui d'une carrière (le pas Bayard) qui en est trẻs proche el qui ajpartient à la zone inférieure (le calcaire de Jaumont).

Il résulte de ce fait que, le soc de la charrue ayant remué toute la parlie meuble fossilifère, la localifé se troure presque entièrement épuisée, ayant élé très souvent explorée. 
indications précises de leur lieu d'orignine, nous sommes parfaitement renseignnés sur l'horizon auquel ils appartiennent.

L'étude des genres nous a imposé des recherches très multipliées et obligé à produire de nombreuses observations, qui trouveront leur place à la tête des genres qui les ont nécessitées.

Toutefois nous ferons observer que dans certains genres la variabilité des espècos est extrème et conduit à des dégénéresecnces qui s’éloignent plus ou moins des types connus; parfois ecs modifications sont si profondes qu'on serait tenté de multiplier les espèces, si, par l'abondance même de ces fossiles, il n'était permis d'établir des séries, qui renferment tous les passages de formes.

Dans la section stratigraphique, il a été démontré que les parties limitrophes entre la Meuse et la Moselle présentent exactement la même faune, parfois localement accompagnée de quclques espèces nouvelles et fort rares; nous en possédons trois que nous avons cru ne pas devoir négliger et dont nous produisons la deseription, bien qu'èles n'appartiennent pas à la Hoselle.

\section{APERGU GÉRERL IES CORPS ORGANIÉS FOSSILES.}

\section{$1^{\circ}$ VERTÉBRES.}

Les vertébrés ne nous ont rien présenté de particulier parmi les Sauriens: quelques rares dents très fragiles. Les poissons ont fourni quelques débris plus nombreux: un fragment de mitchoire d'Ischiodus analogue à ceux que nous avons signalés pour le grès de Hettange, un fragment d'Ichthyodorulites, deux dents de Strophodus, assez communes et parfaitement conservées, enfin des dents microseopiques fort rares de Saurichthys, Iybodus et Gyrodus.

\section{$2^{\circ}$ MOLLUSQUES.}

(a) CÉpiialopodes.

Les Bélemnites sont en général fort rares et ne se rencontrent que dans les couches marneuses, dont le tassement irrégulier a déterminé la rupture des

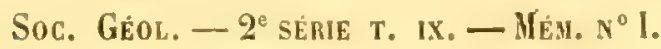


fossiles. Une espèce nouvelle, douée d'un long sillon ventral et d'un commencement de sillon dorsal caractérise la zone inférieure.

Le genre Nautile est représenté par une-seule espèce, fort rare et dont nous ne connaissons qu'un seul exemplaire complet.

Pour les Ammonites, l’A. Blagdeni, caractéristique du calcaire subcompacte, a été trouvé exceptionellement dans un grès calcaréo-marneux avec l'Ostrea acuminata (de grande taille) dans les environs de Hayange; nous avons quelques doutes sur la présence de l'A. Inmphriesianus dans la zone inféricure, qui nous a été communiqué avec l'indication du plateau de Longwy et parait devoir appartenir à l'assise sous-jacente, les calcaires à polypiers.

Les A. subfurcatus Schl. (niortensis, d'Orb.), Parkinsoni et quercinus, nob. caractérisent chacun une zone spéciale.

Pour la zone moyenne, nous citerons deux espèces nouvelles pour la paléontologie française et qui se trouvent indiquées pour la zone moyenne du Wurtemberg: A. neuffensis, Opp .et A. deltafalcatus, Quenst.

\section{(b) Gastéropodes.}

Les genres Turritella, Vermetus, Melania, Nerinea, Tornatella, Acteonina, Natica, Bourgelia, Nerita, Pagodus, Phorus, Trochus, Purpurina, Straparolus, Solarium, Turbo, Pleurotomaria, Alaria, Chiton, Patella, Emarginula et Dentalium, se produisent avec plus ou moins d'abondance et ne sauraient être rangés suivant le nombre des espèces qu'ils renferment. Presque tous ces gastéropodes proviennent des Clapes et possèdent leur test avec tous ses ornements; cette circonstance nous a permis de rechercher la structure intérieure de certaines coquilles et nous avons eu, une fois de plus, la confirmation de l'observation que nous avons eu l'occasion d'exposer dans nos études sur le lias: que les columelles internes peuvent servir de guide certain dans le classement des fossiles et bien mieux que les caractères tirés de la forme de l'ouverture trop souvent oblitérée ou cassée.

On trouvera à la tête de quelques genres les observations critiques qui ressortent de leur étude ; les principales concernent les genres Chemnitzia, Melania, Bulla, Acteonina, ete.

Nous avons trouvé quelques rares débris mieroscopiques de Chiton dans une couche marneuse imprégnéc de sulfure de fer ; cette mention n'a d'autre intérêt que de signaler une nouvelle station de ee genre, si rare dans les dépôts jurassiques; nous avons remarq̨ué que ce fossile se présente dans l'oolithe avec les mêmes conditions que dans le lias, lorsque la roche est fortement pénétrée de sulfure de fer. 
(c) ACERIIALES.

Les genres Gastrochena, Teredo, Pholadomya, Ilomomya, Psammobia, Arcomya, Pleuromya, Gresslya, Thracia, Saxicava, Isodonta, Opis, A starte, Cypricardia, Cardila, Lucina, Corbis, Cardium, Iteltangia, Isocardia, Nucula, Lada, Trigonia, Arca, Cucullar, Pinna, Nytilus, Limes, Lima, Avicula, Gervillia, Perna, Pecten, Plicatula, Ostrea et Anomya sont très variables quant au nombre des espèces et à leur abondance, qui peurent servir à spécifier certaines localités. Ainsi les Clapes se distinguent, entre toutes, par le nombres des genres et la multiplicité des espèces; tous les acéphales possèdent leur charnière et la belle conservation qui jusqu'ì présent semblait exclusive aux fossiles terliaires.

Les Gastrochènes, les Tarets, les Saxicuves, les Modioles, et les Lithodomes, ainsi que certaines Arches vivaient réunis dans les polypiers. Dans des loges creusées par un perforant nous avons trouvé accidentellement une Lime et un Peigne, qui, obligés dans leur développement de se modeler suivant les parois de leur étroite demeure, ont pris ainsi la forme de leurs devanciers.

Les Arehes et les Cucullées perforantes présentent les mêmes caractères que les espèces vivantes corallivores: le champ ligamentaire est très étroit, linéaire, et les erochets exigus sont comme atrophiés et loujours rep iés en dedans.

Quelques genres se distinguent par une abondance extrème d'espèces et une grande variété de formes; nous signalerons en particulier le genre Lucina, qui passe de la forme aplatie à la globulaire; il en est de même pour le genre Astarte dont quelques espèces sont comme laminées; les genres Corbis et Cypricardia sont remarquables par leurs formes, comme les Pleuromya, les Mytilus, les Pecten et les Ostrea le sont par leur abondance.

Deux espèces de Saxicaves sont ornées de stries rayonnantes granuleuses comme les Areomyes, les Pleuromyes et les Greslsyes et que nous avons observées sur une Panopée de Dax; il est probable que ces ornements, d'ailleur's très cadues, se retrouveront sur d'autres espèces, toutes les fois que le test se présentera dans toute son intégrité.

Les Trigonies du Bathonien ainsi que la plupart de celles que nous possédons du Bajocien inférieur sont donées d'une enveloppe pseudo-épidermique, figurant un tissu très serré, à mailles carrées ou rhomboédriques selon les espèces et qui recouvre toute la surface; ces ornements ne sont visibles qu'avec une fortc loupe ou mieux au microseope.

Les Cucullées ont leurs dents longitudinales finement striées transversalement, caractère qui se retrouve sur les dents des espèces vivantes.

Enfin pour les Anomyes que nous signalons dans le Bathonien nous avons trouvé les valves inféricures munies de leur orifice caractéristique. 


\section{(d) BRaCHYopodes.}

Les Rhynchonella, IIomithyris, Terebratula, Thecidea et Crania sont les seuls représentants de celte famille.

Les genres Rhynchonella et Terebratula, plus abondants en nombre qu'en espèces, foisonnent à tous les niveaux et dans toutes les localités; quelques espèces sont propres à certaines zones, comme nous l'avons exposé daus la stratigraphic

Le genre Itemithyris représenté par une seule espèce se montre fort rare dans la zone inférieure el la moyenne, et sc troure très commun dans la supérieure.

Les genres Thecidea et Crania sont excessivement rares.

\section{ARTICULÉS.}

\section{(a) crustacés décapodes.}

Nous avons trouvé, dans les marnes de Fontoy, quelques débris indéterminables de erustacés brachyures; pour les Palinures, le genre Glyphea se montre assez rare dans la zone inférieure.

\section{(b) CRUsticÉs OSTRicodes.}

Les fossiles de cette famille sont généralement aboudants dans les dépôts marneux, qui ont, dès le principe, subi un tassement suffisant pour empêcher le passage des courants acidules; abondants à Fontoy, ils sont fort rares ou mème ont complètement disparu dans la majeure partie des localités que nous avons explorées. Accompagnant constamment les foraminifères, ils ont, comme ceux-ci, subi les mêmes phases d'abondance ou de desiruction.

Si l'espace et les circonstances le permettent, nous les publierons dans ce travail ; en cas contraire nous les produirons à la suite des foraminifères.

\section{$4^{\circ}$ ANNÉLIDES.}

Les genres Galeolaria et Serpula sont partout abondants, principalement dans la zone moyenne, ainsi que le genre Ditrupa qui accompagne les foraminifères de Fontoy. 
¿̈ BRTOZOARES.

Le Bathonien renferme les grenres Diustopora, Iieleropora, Stomaloport el Berenicea, dont toutes les especes, sanf une, ont été classées et publiées frar J. Haime, d'après nos échantillons.

jous avons recucilli a Fontoy des spicules microscophiques calcaires ou siliceux. dont les uns en forme d'hamegon se rapprochent de ceux du genre 1 strophyton, et les autres hémisphériques semblent se rapporter au gene Chirocioul.

\section{$6^{\circ}$ RADIAIRES.}

Dans les échinodermes, les genres à formes irrégulières sont beaucoup plus abondants en nombre que ceux à formes régulières: les genres Clypous, Pygurus, Echinobrissus et Hyboclypus, pour les uns, Pedina, Diadema, Acrosalenia pour les autres.

Les Clypeus abondent dans les zones inféricures et moyennes; les Myboclypus, fort rares dans ees zones, sont au contraire très communs dans la supcrieure; les Echinobrissus el Pedina paraissent propres à la zone inférieure.

Les genres Diadema et Acrosalenia, le premier assez abondant, le second fort rare, n'ont encore été trouvés que dans la zone moyenne.

Dans les rayonnés, le genre Eugeniacrinus est fort rare, et les débris du genre Pentarrinus abondent dans toute les localités.

Les articulations d'un crinoïle, que nous rapportons au genre Ophyoderma, se trouront à toutes les hauteurs; mais elles se présentent particulièrement à Fontoy, où une couche, à 2 mètres sous l'aire de la gare, en est littéralement pétrie; le lavage des marnes donne un résidu uniquement formé de ces débris, tant du disque que des bras.

Nous possédons de Ranguevaux (niveau ilu calcaire de Jaumont) un magnifique astéroïde, qui se trouve dans un état de conservation fort rare; nous l'arons classé provisoirement dans le genre Asteracainlhion.

On nous a communiqué un autre astérö̈de, non moins bien conservé, mais sans indication de provenance; la roche qui le supporte, et qui se montre identique à celle de Ranguevaux, permet de le rapporter à la même zone; la constitution du fossile le rapproche complètement du genre Astropecten.

\section{$7^{\circ}$ ZOOPIIYTES.}

Les genres de cette famille sont peu nombreux, en raison du caractère marneux qui prédomine dans la majeure partie des assises; on ne peut les reneontrer 
que dans les dépôts essentiellement calcareux; les genres Anabacia et Montlivaltia paraissent propres à la zone supérieure et se trouvent en abondance sur les liauteurs d'Auboué à Gorze, dans les environs de Conflans et de Jarny et dans les premières limites du département de la Meuse.

l.es genres Isastrea et Thamnastrea ne se trouvent qu'aux Clapes.

\section{$8^{\circ}$ FORAMINIFĖRES.}

Nous sommes convaincus que les foraminifères ont dû, dans le principe, se trouver "en abondance dans toutes les couches et dans toutes les localités; la perméabilité de certains dépôts aux courants acidules les a laissés disparaitre en majeure partie, attendu que partout nous avous trouvé des traces de ces fossiles.

La localité de Fontoy est exceptionellement remarquable par l'exhubérante abondance des fossiles microseopiques.

L'étude de ces fossiles et leur classement définitif ne sont pas terminés, mais nolss pouvons, dès à présent, indiquer les genres que nous avons reconnus : Orbulina, Glandulina, Nodosaria, Dentalina, Frondicularia, Lingulina, Iarginulina, Ataxophragmium, Vaginulina, Webbina, Cristellaria, Flabellia, Robulina, Nonionina, Rotalina, Rosalina, Truncatulina, Uvigerina, Polymorphina, Biloculina, Spiroloculina, Tiloculina, Sphoroidina, Quinqueloculina.

Nous ferons remarquer que dans le Bathonien, de mème que dans le lias, l'ordre des Entomostègues manque complètement; que les ordres des Monostègues et des Enallostègues ne sont représentés chacun que par un ou deux genres; que les ordres des Stichostègues, des Hélicostègues et des Agathistègues possèdent de nombreux genres et une grande abondance d'espèces.

\section{$9^{\circ}$ ANORPHOZONIRES.}

Les fossiles de cette famille sont en général fort rares et nous n'en possédons que quelques ćchantillons, provenant des Glacis de Longwy et des Clapes.

Nous possédons de Fontoy des spicules siliceux, aciculaires, qui présentent la plus grande analogie arec ceux que renferment les spongiaires à tissu fibreux. 


\section{TROISIEME PARTIE.}

\section{DESCRIPTIONS DES ESPËCES.}

VER T ÉBRÉS.

SAURIENS.

Nous ne connaissons qu'une seule dent régulièrement conique et que nous rapportons au genre Ichthyosaurus pak son analogie avee celles que nous trouvons dans le lias.

Localité : Les Clapes, fort rare, (collection de $\mathrm{Y}$. Colliez).

\section{POISSONS.}

Nos recherches de foraminifères nous ont fait trouver plusieurs dents, plus ou moins microscopiques; les unes sont coniques et finement aiguës, les autres obtuses à leur sommet, lisses ou striées.

Localité : Fontoy, (1) dans plusieurs couches, 1-3- 10 en général fort rare.

(1) Teryuem. 1 Mémoire sur les Foraminiferes de l'Oolithe. Ialroduction, p. 56. Un tableau indique les divers niveaux de la prise des marnes á Fonloy. 
Hybonus neticulatus, Ag. Pl. 1. Fig. : 3, 4 et 5.

Agassiz, Poissons fossiles t. III, pag. $50, \mathrm{pl} .9$, fig. 1 à 9 .

Dimensions : longueur 40 millim. ; largeur inférieure 30 millim. ; largeur supérieure 22 millim.; épaisseur 15 millimètres.

De toutes les espèces figmrées par Agassiz, celle qu'il a indiquée pour le lias, concorde le mieux avec notre échantillon, malheureusement très incomplet.

Cette espèce est irrégulièrement quadrangulaire; ses côtés sont ornés de 11 côtes obtuses qui vont en diminuant de grosseur d'arrière en avant; le dos est muni d'une arête arrondie, et le côté ventral plié à angle droit est orné d'un grand nombre de fines côtes régulières, traversées par des côtes plus fines, qui donnent à cette partie un aspect réticulé.

Localité: les Clapes. Fort rare.

Strophodus loxgidens, Ag. PI. 1, fig. 1-2; grandeur naturelle. Agassiz, Poissons fossiles, t. III, p. 117, pl. 16.

Dimensions: longueur 41-32 mill.; largeur 23-18. mill.

Les dents sont régulièrement trapézoïdales et généralement bien conservées; l'une d'elles montre les pores irréguliers de la dentine; une autre plus petite est recouverte d'un émail noiràtre, qui ne laisse voir les pores que sur le pourtour.

Localités : les Clapes, Gravelotte, Fontoy. Assez commun dans la $1^{\text {re }}$ localité.

\section{Iscilyodus.}

Nous rapportons à ce genre un fragment d'os qui appartient à la mâchoire inférieure et qui présente beaucoup d'analogie avec des fragments semblables, que nous avons trouvés à llettange; il est probable que celui-ci appartient à l'I. Tessoni que Agassiz a dénommé pour le calcaire de Caen.

Localité: Gravelotte, dans les marnes bleues. Fort rare.

\section{IsciYyodus.}

Un de nos collègues, M. l'abbé Friren, nous a communiqué une dent qu'il a trouvée récemment à Gravelotte et que nous rapportons à ce genre par sa constitution et son analogie avec celles que nous possédons de Hettange : elle est convexe, irrégulière, brillante, d'une couleur noire, flamblée de taches blanches et douée d'une dentine très serrée, quoique fibreuse.

Localité: Gravelotte, Calcaire marneux. Fort rare. 


\section{MOLLUSQUES.}

CEPHALOPODES.

Belemites gighyteus, Schl.

Schlotheim, Petrefucten hunde, 1. I, p. 43.

Localités: Gravelotte, Fontoy, Longwy (les glacis). Fort rare et brisé, plus commun et de grande raille dans le calcaire subcompacte.

BeLEMITES CAMAlculats, Schl.

Schlotheim, Petrefacten kunde, 1. 1. P. 49.

Celte espèce, généralement petile, se présente dans le Bajocien supérieur ; assez abondante dans le calcaire à polypiers, elle devient fort rare dans le calcare subcompacte, pour se montrer plus abondante dans le Bathonien; toujours fragmentaire, clle se confond facilement arec le B. sulculus.

Localités: les Clapes (fort rare), Gravelotte, Fontoy, Longwy (glacis); assez commun.

Belemites sulcatus, Mill.

iiiller. Trents of the Geol. Soc., 1. II, p, 89, pl. Vlll. fig. 3.

Localités: Gravelotte (marnes), Longwy (glacis); assez commun.

Belenites Jacouoti, Ter'q. el Jourd. Pl. I, fig. 6-7-8-9;

B. testa elongata, compressa, antice angustata, postice attenuata, apice subacuminata, lateribus bisulenta, sulco uno longo postice evancscente, altero brevi prope alveolum incipiente.

Dimensions: longueur $\$ 0-\$ 0$ mill., largeur antérieure $7-6$ mill., largeur ventrale $9-8$ mill.

Rostre allongé, comprimé, fusiforme, étroit en avant, un peu élargei dans le milieu, puis fortement rétréci en arrière et terminé en pointe subaiguë, pourvu de chaque côté d'un sillon profond, l'un ne commençant qu'avec l'élargissement de la coquille, l'autre très-court près de la partie alvéolaire.

Celte espèce présente la forme générale du $B$. hastatus, et en diffère par la compression qui règne sur toute sa hanteur, par sa partic postérieure plus atténuée et par son grand sillon, qui, plus profond et plus étroil, descend plus près de la pointe.

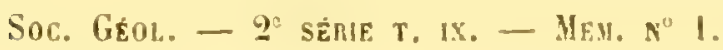

(j. 
Elle s’éloigne du $B$. conaliculatus, quui est réguliẻrement conique à la partic postérieure et par son sillon qui descend jusqu'à la pointe.

Les rapports sont plus nombreux entre le B. Jacquoti et le B. Coquandus, d'Orb., de l'Oxfordien, par la forme, la compression, les deux sillons, la disposition du grand sillon; mais dans l'espèce oxfordienne les deux sillons sont égaux.

Localités : Rampe de Montigny sur Chiers, Longwy (glacis); assez commun.

Nautilus excavatus, Sow.

Sowerby. Mineral Conchology, p. วั43, pl. \$29, fig. 1-2, d'0rbigny.

Paléontologie francaise, p. 154. pl. 30.

Localité: les Clapes; fort rare (Collection de I. de Lambertye).

Angonites Parkinsoxi, Sow.

Sowerby. Mineral Conchology, p. 342, pl. 307. - Ammonites interruptus,

Brug. d'Orbigny, Prodome, t. 1, p. $261, \mathrm{n}^{\bullet} 16$.

Cette espèce constante dans ses ornements montre à ses différents âges quelques variations dans l'épaisseur de ses tours; la coquille est plus ou moins aplatie et les tours sont plus ou moins larges; au diamètre de 20 à 25 cent. la plupart des côtes ont disparu.

Dans la localité des Clapes, cette coquille se présente exceptionellement avec son test parfaitement conservé; dans toutes les autres on ne trouve que des moules.

Localités: les Clapes, Gravelotte, Fontoy, Côtes de Jay (vallẻe de Mance); généralement abondant dans toute la zone.

Amronites subfurcatus, Schl.

Schlotheim, Petrefacten Kunde, p. 73. Quenstedt, Jurt, pl. 53, lig. 19. - Ammonites niortensis, d'Orbigny, Paléontologie française, p. 372, pl. 121, fiğ. 7.

Cette espèce a été parfaitement décrite et figurée par d'Orbigny, qui l'a classée dans le Bajocien et n'a pas mentionné l'A. subfurcatus. Elle se trouve à Bayeux et s'y montre accompannée des $A$. Sowerbyi et Parkinsoni, qui, dans notre département, sont séparés par plus de 40 mètres de roche, et par conséquent chacune peut servir à caractériser une zone distincte: l'A. Sowerbyi est propre au Bajocien inférieur, l'A. subfurcatus au Bathonien inférieur, et l'A. Parkinsoni au Bathonien moyen.

Bronn (Index paloontologicus) indique un subfurcatus, Schl., et le considère comme identique avec le Parkinsoni, Sow; puis un autre subfurcatus, Ziet., qu'il rapporte au cordatus, Sow.

Localités: Longwy (les glacis); assez commun. Vallée de Montvaux, près d'Amanvillers, fort rare. 
AMrovites Blagden, Sow.

Sewerby, Nineral Conclology, p. 231, 1). 201.

Cetle espece, qui semble, par son abondance et par lid grande dimension que possèdent les coquilles, être caractéristique du calcaire subcompacte (Bajocien supérieur), a élé trouvéc dans un calcaire gréseux arec l'o. acuminata, de trèsgrande taille.

Localité: près du bois entre Ranguevaux el Hayange, fort lare.

Amoxites deltafalcatus, Quenst.

Quenstedt, Jura, p. 399, pl. 3̈3, fig. 7-8.

Cette espèce se trourc bien représentée, mais incomplétement décrite.

Coquille comprimée, ornée de côtes droites et simples dans le jeune âge et de plis peu marqués dans l'adulte; spire formée de cinq tours comprimés, obliques près de la suture, se recouvrant au quart de leur diamètre; dos arrondi et muni d'une carène obtuse; ouverture subquadrangulaire.

Cette espice au premier aspec: ressemble à 1'A. primordialis du lias supérieur et s'en éloigne par son ombilic largement ouvert el par la disposition de ses cloisons. Elle diflère de l'd. Murehisone, Sow, qui a ses tours coupés presque verticalement près de la suture, dont l'ombilie estplus étroit, les tours se recouvrant à la moitié de leur diamètre et dont les cótes dans le jeune âge sont trèsélerées et bifurquées.

Localité : les Clapes, fort rare (Collection de II. de Lambertye).

$$
\begin{aligned}
& \text { Amoxites Tessomanus, d'0 b. } \\
& \text { D’0rbigny, paléont. frane., p. 392, pl. 130, fig. 1-2. }
\end{aligned}
$$

Nous avons trouvé un fragment d'un exemplaire adulte, qui présente les caractères propres à l'espèce; nous la possédons également du Bajocien inféricur des environs de Hetz, où elle se présente avec une certaine abondance.

Localité : Ies Clapes; fort rare.

Amsonites necfrexsis, Opp.

Ammonites Parkinsoni gigas, Quenstedt, Céph. p. 143, pl. 11, fig. 1. Ammonites neuffensis, 0ppel., Form. du Jura, p. 378, \$33, n" 48.-Ammonites posterus, Seebach., Jura, p. 130, pl. 10, lig. 3.-Ammonites neuffensis,

Opp., Schlocnbach, Paloontographica, vol. 13.-Cont. pateont., p. 27, pl. 28, lig.

Cette espèce, qui parait n'ètre qu'une variété de l'A. Parkinsoni, Sow., dont elle possède le sillon dorsal et la même disposition dans les lobes et les selles, en diffère par des tours beaucoup plus renflés et plus embrassants, qui rendent l'ombilic d'autant plus profond et plus étroit. 
Les côtes, toutes égales prìs du dos, deviennent plus espacées et plus saillantes près de l'ombilic; celles qui s'arrêtent an milieu des côtés, au lieu d'être simples comme dans le Parkinsoni, sont par 4 ou 5 entre chaque grande còte.

Localité: Cutry, près de Cons-la-Granville, fort rare (Collection de M. Colliez).

AMHOXITES Quercinus; Terq. et Jourd.

Pl. 1, fig. 10-11-12-13. - La figure 10 est moitié de grandeur naturelle.

A. testa discoidea, compressa, dorso rotundato, costato, anfractibus depressis, juvenile duas testo partes, adulte dimidiam obtegentibus, umbilico Iato, apertura ovali, 30-33 costis obtusis, dorso bi vel trigeminatis, ad umbilicum simplicibus, inflatis, in medio evanescontibus ornata.

Coquille discoüdale, compriméc, non carénée, ornée de 30 à อ̊ŏ côtes qui se doublent ou se triplent en passant sur le dos. Dans le jeune âge (jusqu'au diamètre de 5 cent.) les côtes sont saillantes et d'épaisseur à peu près égale sur tout leur parcours; plus tard elles se renflent sur le bord de l'ombilic et, s'atténuant vers le milieu du tour, s'accentuent un peu plus sur le dos; enfin dans l'adulte (au diamètre de 20 cent.) les côtes sont larges et obtuses vers l'ombilic, effacées au milieu du tour: mais sur le dos elles sont assez visibles quand le test est enlevé. Spire formée de tours se recouvrant sur les deux tiers de leur largeur dans le jeune âge, landis que, dans l'adulte, le recouvrement diminue de moitié; aussi l'ombilic s'élargit-il avec l'âge. A tout âge le tour est peu courbe au milieu; mais il le devient subitement au voisinage du dos et de l'ombilic; la partie plane dont il est question augmente d'importance avec l'âge.

Lobes très-découpés; plusieurs des lobules imitent la feuille de chêne.

Selles profondes, peu larges, de plus en plus inclinées sur la spire moyenne d'enroulement à mesure qu'on les prend du dos à l'ombilic; cependant tout près de l'ombilic, à la partie courbe, elles se redressent un peu, mais à cet endroit elles sont presque atténuées.

$1^{\text {er }}$ lobe droit très-court. $-1^{\text {re }}$ selle peu importante. $-2^{\text {e }}$ lobe un peu massif, découpé par une selle secondaire de peu d'importance, fortement découpé par de petites selles sur les côiés. - qe selle inclinée de quelques degrés, très-profonde ; ses ramifieations découpent profondément les lobes adjacents; ces lobules se l'emarquent notamment au fond de cette selle par leur ressemblance parfaite avec des feuilles de chêne. - $3^{e}$ lobe fortement découpé par trois ou quatre selles secondaires. - 3e selle inclinée de 30 degrés, très-ramifiée, moins profonde que la précédente. - $4^{\mathrm{e}}$ lobe découpé profondément. - $4^{\mathrm{e}}$ selle presque perpendiculaire à l'enroulement, par conséquent dirigée suivant un rayon de la spire; elle va presque rejoindre la deuxième selle. - 乌̌e selle dirigée suivant un rayon. A ce moment les lobes deviennent de plus en plus petits; les selles, en suivant cette diminution sont moins obliques. 
Cette espece, par sa forme se rapproche de A.jurassica que Quenstedt (Jura, p. 771, pl. 94, fig. 20) indique pour le P'ortlandien; elle en differe par un moins grand nombre de còtes et par ses sutures excavées.

lapports at différences. Celte espéce est roisine de l'dmmoniles arbustigerus par sa forme générale, ses lobes et son dos.

Elle est moins grlobuleuse; les côles sont plus aceentuces; l'ombilic moins profond devient beaucoup plus large dans l'adulte; les deux plans inclinés qui aplatissent chaque côté du tour forment un biscau plus marqué. Les lobes sont beaucoup plus découpés par les selles principales et surtout par les selles secondaires,

Enlin l'inclinaison variable et continue des selles est tout à fait caractéristique pour celte espéce, et l'éloigne de foutes les Ammonites que nous connaissons.

Observation. Celle espèce, quoique nouvelle, a déja élé recueillie; dans la collection de M. Jaceard, au Locle (Suisse), nous en avons vu un échantillon que II. Desor a menlionné dans son Jura neuchâtclois; ce paléontologiste l'a classé sous le nom d'A. wurtembergicus, Opp., en faisant remarquer qu'it rérnait un peu de confusion dans ces fossiles, et qu’il y voyait plusieurs espèces distinctes. L'échantillon de H. Jaceard ne laisse voir que peu de surface des lobes, de sorte que notre caractère spécifique aurait échappé à $M$. Desor, s'il avait décrit le fossile ¿u'il a considéré conme une variété de l'A. wurtemberyicus.

Gisement et localités. Cette Ammonite se trouve des que se montre le Terebralula lagenalis, e'est-à-dire dès le commencement de la $3^{e}$ zone, et monte dans cette zone jusqu'au sommet, tandis que la Térébratule caractérisée par sa grande taille et sa forme rhomboédrique ne se trouve qu'à la base. Au sommet de la zone (limites de la Moselle et de la Meuse, sur la route d'Étain à Briey), cette Ammonite se trouve avec le Rhynchonella concinna, - quadriplicala, Gresslyn lunulata, Sow. Sp.

y. Colliez a trouvé celte espèce sur le platcau qui domine Cutry, près de Longwy.

\section{GASTEROPODES.}

Ternitella clapessis, Terq. et Jourd., M. 1, fig. 14.

T. testa elongata, conica, anfractibus tetragonis, quadricinctis, cingillo postico minore, stricte clathratim striala, suturis latis, apertura quadrilatera, integra.

Dimensions : longueur 33 millim., largeur 8 millim., rapport du denier tour, 20 l). 100.

Coquille incomplète, allongée, régulièrement conique, formée de tours carrés, ornée de quatre côtes longitudinales, la postérieure plus étroite que les autres, ę de stries verticales très-serées; sutures larges, ouverture quadrangulaire entière. 
Nous avons pu nous assurer que les columclles internes présentent bien les caractères du genre (1).

Localité : les Clapes; fort rare (collection de M. de Lambertye).

Turritella inornata, Terq. et Jourd. PI. 1, fig. $130-16$.

T. testa elongata, conica, lovigata, anfractibus numerosis, convexiusculis, suturis excavatis, apertura qualrangulari, basi plana, multicingulata.

Dimensions : longueur totale, 38 millin., longueur de l'échantillon, 21 millim., largeur, 6-\$millim., rapport du dernier tour, 20 p. 100.

Coquille incomplète, allongée, conique, lisse, brillante, formée de tours nombreux, légèrement convexes, à sutures larges et profondes; onverture quadrangulaire à angles arrondis, columelle verticale, base plane, ornée de plusicurs stries concentriques.

Ne possédant qu'un seul échantillon, nous n'avons pu, vu la fragilité de la coquille, connaitre ni la disposition ni la formè des columelles.

Localité : les Clapes; fort rare (Collection de I. Colliez).

Gexre Vermetus, Adanson.

Ce genre, qui parait avoir été dans les temps anciens aussi abondant que dans nos mers actuelles, se distingue très-difficilement des Serpules et a été presque toujours confondu avec elles; nous croyons devoir attribuer à ce fait la rareté des espèces signalées dans les terrains secondaires.

bronn (Index) lange ce genre après les Dentales.

Vermetus costulatus, Terq. el Jourd.

Pl. 1, fig. 17-18; la figure 18 est grossic 4 fois.

$V$. testa exili, diversim flexuosa, adulta libera, circiter costulis longituanalibus et striis transversalibus clathrutris ornata.

Coquille grêle, flexueuse, libre dans l'adulte, ornée tout autour de petites côtes longiludinales et de très-fines stries transversales.

Localité : les Clapes ; très-commun.

Melania vittata, Phill., PI. I], fig. 1-2-3.

Melania vittata, Phillips, Geol. York., p. 116, pl. 7, fig. 1\%. - Chemnitzia viltala, d'Orb., d'Orbigny, Prodome. T. I, p, 298, $\mathrm{n}^{\circ}$ 29. - Chemnitzia vittata, Phill., sp. Horris et Lycell. Descript. des moll. de la Gir.ool. de l'Angl. p. 14, pl. 31, lig. 10,

Cette espèce n'étant pas indiquée dans la Palćontologie francaise, nous arons cru devoir la figurer.

(1) Voyez Terquem, Paléontologie du grès de II cllange, art. Turritella, p. 33 
La figure et la description données par Morris et Lycett pour cette coguille du Bathonien se rapportent exactement à nos ćchantillons; cependant nous avons reconnu que la columelle est plus oblique et plus conique que ne le montre le dessin reproduit par ces auteurs, et la carène antérieure est marquée sur le retour dul labre.

Nous ferons remarquer que les biseaux sont à peine indiqués dans le jeune âge; avec le développement de la coquille, ils deviennent normalement plus prononcés.

Nous avons usé sur la meule un de nos échantillons des mieux conservés, et nous avons obtenu la disposition intérieure des columelles, qui nous a démontré, une fois de plus, que les Mclania et les Chemnitzia devaient posséder la mème organisation dans leurs viscères; dans ces deux genres, les columelles se trouvent soumises aux mèmes lois de relation: chacune d'elles forme un còne tronqué, dont la base est en arrière et disposée symétriquement sous un angle de $10^{\circ}$ par rapport au grand axe de la coquille (1).

Nous possédons des échantillons dont la taille est double que ceux que nous ayons figurés.

Localité : les Clapes; très-commun.

Melayia Bellona, d'Orb.

Chemnitzia Bellona, d'Orbigny, Paléont. franc., 1. 53, pl. 241, fig. 1-2 (Bath.). Localités: Longwy (glacis), Longuyon. Moules assez communs.

Mélayia niortensis, d'Orb.

Chemnitzia niortensis, d'Orbigny, Paléont. franc., p. 48, pl. 242, fig. 1-2 (Bath.). Localité : Longwy (glacis). Moules fort rares.

Melayia xormaniana, d'Orb.

Chemnitzia normaniana, d'Orbigny, Paléont. frane., p. 40, pl. 238, fig. 4-6.

Nos échantillons, quoique un peu plus petits que ceux figurés par d'Orbigny, se rapportent exactement à ses dessins et à ses deseriptions.

Nous possédons plusieurs moules qui nous semblent appartenir à cette espèce, autant par la forme de l'ouverture que par quelques fragments du test, qui est lisse et brillant.

Localités: les Clapes; très-commun; Gravelotte, (marnes); assez rare, mẻme à l'état de moule.

Melania exilis, Terq. et Jourd. I'. 2, fiğ. 4-כ̋.

M. testa longissima, angustata, anfractibus numerosis, lovigatis, antice convexiusculis, postice subplanis, suturis incisis, angustis, apertura oblique ovatr.

(1) Vogez Terquem, Paléontologie de Hellange, art. Melauia, p, 36. 
Dimensions : longueur (présumée) 90 mill., diamètre près de l'ouverture 7 mill., diametre du $3^{\circ}$ tour 6 mill., rapport du dernier tour 5 p. 100.

Coquille incomplète, très-allongée, étroite, formée de tours nombreux, croissant très-faiblement, lisses et marqués de plis inégaux d'accroissement, légèrement renflés en avant, déprimés en arrière ; ourerture ovale-aiguë, labre très-mince, bord columellaire droit et épais ; base très-déclive et munie d'une mince callosité; sutures incisées, peu profondes.

Nous rapportons à cette espèce des moules dont les tours croissent très-lentement et qui présentent les mêmes dimensions que celles que nous avous indiquées.

Cette espèce, qui ne se rapporte à aucune de celles qui ont été publićes, a la disposition genérale propre à certaines Nérinées, pour le faible développement que prend la coquille à chaque tour ; nous arons présumé la longueur totale de la coguille en cumtinuant l'angle formé par les derniers tour's.

Localités: les Clapes; fort rare et fragmentaire; Gravelotte (marnes); assez commun, mais à l'état de moule.

Nenimea lamisa, Terq. et Joumd. Pl. 1, fig. 19.

N. testa clongata, conica, turriculata, anfractibus planis, juvenile cingulis tribus caqualibus, adulte cingulo antico sensim erescente, laminato, clato, ornata, sutwis excavatis.

bimensions: longueur 23 mill., largeur 8 mill., rapportdu dernier tour 20 p. 100.

Corquille allongée, conique, turriculée, formée de tours non saillants, ornée dans le jeune âge de trois côles longitudinales égales, l'antérieure croissant insensiblement et devenant dans l'état adulte lamellaire et élevée; sulures profondes, ouverture guadraugulaire.

Cette espèce par ses opnements se distingne de toutes ses congénères. Ne possédant qu'un seul exemplaire et qui nous a été confié, nous n'avons pu nous livrer à la recherche des eloisons intemes.

Localité : les Clapes; fort rare (Collection de H. de Lambertye).

Nerinea claves, Desl.

Eudes Deslongchamps, Mém. de la Soc. linn. de Norm., t. 7, p. 183̈, pl. 8, fir. 28-29.

Localite : les Clapes; fort rare (Collection de M. de Lambertye).

Nerinea fuxiculosi, Dest.

Eudes Deslongchamps, Mém. de la Soc. linn. de Norm., p. 186, pl. 8, fig. 30-з2.

Localités: Gravelotte (marne), Gorze (cimetière); fort rare.

Gexre Acteonina, d'Orb.

D'Orbigny, en démontrant qque les Cônes du lias mojen étaient de véritables Actéonines, parce que leur test intérieur n'était pas résorbé, a appelé l'attention 
sur Ia classification de certains fossiles qui aflectent des formes diverses; si les uns, comme nous venons de le dire, ressemblent à des Cỏnes, d'autres présentent exactement la forme extérieure des liulles.

D'Orbigny (Prodrome), s'appuyant uniquement sur des dessins, a rangé, dans l'oxfordien et avec Ies Actéonines, les Bulles que Roemer indique pour l'oolithe inférieure.

On sait que les detéonines n'ont pas de plis à la columelle et ne résorbent pas leur test intéricur, tandis que les letéons (Tornatelles) possèdent des earactères opposés; ils ont des plis il la columelle et sont doués d'une résorption de test, scmblable à celle que possident les Cônes. Les Bulles semblent être intermédiaires entre ces deux genres; elles ont le bord columellaire sans pli et résorbent fortement leur test intéricur.

Pour nos fossiles, nous arons en soin d'en couper un par le milieu et nous avons vu le test intérieur excessivement mince, mais aussi égal pour tous les tours; de là nous avons pu conclure qu'il fallail ranger ces corquilles avec les Actéonines et non parmi les Bulles, contrairement aux indications rësultant de l'ourerture et de la forme générale de la coquille.

Acteonisa aequalis. Teri. el Jourd. Pl. 2, fig. 6-7; grandeur naturelle.

A. testa tumida, aquali, transversim obsolete plicata, spira abbreviata, sublana, anfroctibus 6-7, infore subacute angulatis, interne excavalis; columella integra, spissa, arcuata, aperture antice ampla, postice angustissima.

Dimensions: hauteur 24-88 mill.; diamètre 13 -23 mill.; rapport du dernicr tour 930 0 0

Coquille renllée, sensiblement égale sur toute sa hauteur, un peu rétrécie en arrière, olnée de plis transyersés el espacés, spire courte et presque plane, formée de 6-7 tours juxtaposés, mumis postérieurement d'un angle subaigu, suivi d'une excavation; ouverture large en avant, très-étroite et anguleuse en arrière; columelle entière, épaisse, arquée.

Localité : Longwy (glacis), très-lare.

Acteonina disuncta. Terọ. el Jouril. Pl. 2, fiğ. 8-9-10; grandeur naturelle.

A. mucleo clongato ovato, subcruali, transversim striato, spira subproducta, anfractibus 7, postice angulo subucutis, disjunctis, apertura antice ampla, postice angusta, columella integra, spissa, arcuala.

Dimensions : hauteur 37 mill.; diamètre 16 mill.; rapport du dernier tour 830 0 0 .

Moule ovale-allongé, sensiblement égal sur toute sa hauteur, strié transrersalement; spire un peu proéminante, formée de 7 tours, comme juxtaposés, disjoints postéricurement et munis d'un angle subaigu; ouverture large en avant, trèsétroite en arrière, columelle épaisse, arquéc.

Localité : Vallée de Hontvaux, près d'Amanvillers, fort rare.

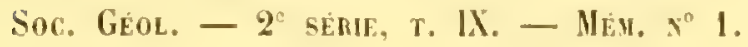


Acteovisi olivacea. Terq. el Jourd. P1. 11, fig. 11, 12; grandeur naturelle.

A. nucleo elongalo-ovato, spira subproducta, anfractibus 6 plicatis, subgradatis, angulo, obtuso, apertura elongata, angusta; columella recta, integra.

Dimensions : longueur 18 mill.; largeur 9 mill.; rapport du dernier tour 7300.

Moule ovale, allongé, sensiblement égal sur toute sa hauteur, spire courte, formée de 6 tours en gradins, anguleux, à angle arrondi, ouverture allongée, étroite; columelle droite.

Localité : Longwy (glacis), très-rare.

Acteonina clapensis. Terq. et Jourd. Pl. 2, lig. $18-14$; grossic 4 fois.

A. testa brevi, ovata, lavigata, anfractibus tribus tumidis, ultimo aliis multo majore, suturis profundis, apertura lata.

Dimensions: longueur 2, 3 millim.; largeur 2 millim.; rapport du dernier tour 9000 .

Coquille un peu plus longue que large, ovale dans son ensemble, formée de trois tours renflés, arrondis, le dernier beatcoup plus grand que les deux premiers, sutures profondes, ouverture large, columelle droite, entière.

L'ouverture presque demi-lunaire rapproche cette espèce des Phasianelles et elle s'en éloigne pour sa columelle allongée et verticale.

Localitẻ : les Clapes, fort rare.

Acteonina ponderosa. Terq. el Jourd. Pl. 2, fig. 1:, 16, 17, grossie; 2 fois.

A. testa clongata, conica, anfractibus sex terelibus, levigatis, ultimo postice paululum impresso, obsolete cingillato, apertura acute-ovata, columella recta, integra.

Dimensions : longueur 9 millım.; largeur 4 mil.; rapport du dernier tour 50010.

Coq̨uille allongée, conique, formée de six tours renflés, arrondis, le dernier légèrement déprimé en arrière, aussi grand que le reste de la coquille, orné de nombrenses stries en ceinture, d'autant plus serrées et plus saillantes qu'elles se rapprochent de l'extrémité antérieure, l'une d'elles plus profonde près de l'angle sutural ; ouverture ovale-aiguë, columelle droite, base arrondie.

Localité : les Clapes, fort rare.

Tonstella cixgllata. Teriq. el Jourd. Pl. 2, fig. 18, 19, 20 ; grossie $\frac{5}{2}$ fois.

T. testa purvula, anfractu ultimo, infla to, subsphorico, sparsime regular iter. cingillato, apertura angusta, acule ovata, columella bicostala, antica costa quam postica majore.

Dimensions : Iongueur du dernier tour 6 millim. ; largeur 4 millim.

Conuille ineomplète, dernier tour renflé, subsphérique, orné de stries trèsकरह 
espacées, divisant la surface en bandes régulières; ouverture étroite, très-aiguë en arrière; columelle munic de deux plis, l'antérieur deux fois plus gros que le postéricur.

Bien que celte coquille soit cassẻe à son extrémité, nous n'avons pas hésité à en faire une espéce, bien caractérisée par ses ornements, la forme sphérique de la coquille et par les plis inégaux de la columelle.

Localité : les Clapes, fort rare.

Bovraeti struti, Sow. sp. PI. 2, fig. 21, 22, 23; grandeur naturclle.

Melania striata, Sow., Sowerby. Mineral Conchology, t. 1. p. 101, pl. 47.

Plusieurs auteurs ont cité cette espèce; tous l'ont classéc différenment et aucun ne s'est appuyé sur un caractère ayant quelque valeur.

Boblaye en a fait une Phasaniella (Ann. des sciences naturelles, 1830).

Lonsdale une Terebra (Geolog. Trans.).

Eudes Deslonchamps a conservé la dénomination de Sowerby (Hém. de lu. Soc. linn. de Norm., 1842, Ném. sur les Mélanies fossiles, p. 221, pl. 12, tig. 3-4), et a ajouté en observation (p. 222): "Comme celte coquille n'a été observée qu'à l'état de moule intérieur, il serait bien possible que l'extrémité inférieure (antérieure) de l'ouverture ne fùt pas continue, mais disjointe el échancrée. Lin ce cas, celte espèce appartiendrait aux Tones, auxquelles elle ressemble d'ailleurs par la tenuité de son test et par les stries en cerelures transverses de ses tours ; elle n'en diflérerait que pour l'élévation de la spire."

D'Orbigny a classé cette coquille, comme Boblaye, parmi les Phasianelles, (Prodrome 1850, t. 1, p. 333, n ${ }^{0} 79$. Callovnic; P. 353, $\mathbf{n}^{0} 124$, Oxfordien. Paléont. franc., p. 322, pl. 324, fig. 13 et pl. 325, fig. 1.).

Le Melania striata est une espèce trop connue pour avoir besoin d'ètre décrite à noureau; elle se présente avec une abondance extrême dans le Bajocien supérieur, le Caleaire à polypiers de la Hoselle; plus rare dans le Bathonien inférieur, elle se trouve encore dans l'oxfordien et mème dans le Corallien.

Avant d'entrer dans la discussion pour savoir à quel genre il convient de rapporter ce fossile, nous avons à présenter une question préjudicielle el nous demanderons, s'il suffit qu'une coquille soit altongée, possède des tours arrondis et soit ornée des stries longitudinales pour qu'elle doive être rapportéc à l'espèce typique établie par Sowerby.

Nous ne le croyons pas, par ces motifs: une coquille du lias inféricur (1), douéc des caractères mentionnés ci-dessus, a été classée parmi les Turritelles, parce que ses columelles internes montraient la disposition propre à ce genre; toutes sont obliques sous un angle de $20^{\circ}$, par rapport au grand axe, (2). Un autre fossile

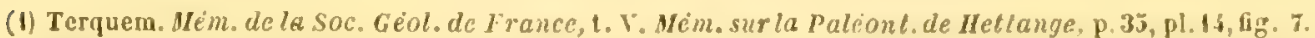
(2) Terquem el Pielle. Mim. de la Soc. Géol. de France, t. VII, Mf m. sur le lias inferieur de l'Est de la France. 
du même terrain est devenu un Cérithe, parce que toutes les columelles coupées dans leur milieu par le grand axe sont disposées en còne, dont la base est du côté de l'ouverture, et montrent une échancrure près de la cloison supérieure.

De ces deux faits nous pouvons déjà tirer cette conclusion, qu'une coquille, le Melania striatu, qui se présente avec des caractires extérieurs identiques dans plusieurs terrains, depuis le Bajocien jusque dans le Corallien, pourrait bien, comme celles du lias, appartenr it divers genres, selon le terrain qui la renferme, si les caractères intéricurs de la coquille pouraient ètre connus.

Examinons maintenant les caractères fournis par les genres auxquels on a cherché à rapporter le Helania striata.

$1^{\circ}$ Le genre Phasianelle a une ouverure enticre, ovale; le labre est tranchant et le côté columellaire épais et en expansion calleuse sur la base. Le test interne est résorbé et les columelles sont arquées, de manière à ce quue le grand axe ne les coupe qu'à leur base, le sommet élant rejeté à gauche, sous un angle de $23^{\circ}$.

Quand des columelles sont ainsi rejetées sur le côté, la cợille n'a pu, en aucune circonstance, être munie d'm ombilic ouvert ou caché par un encroutement du bord columellaire.

$2^{\circ}$ Les Tones, indépendamment de la forme si caractéristique de leur ouverture, ont leurs columelles internes disposées rerticalement, suivant le grand axe, ornées de deux gros plis en torsade et elles se communiquent entre elles par le moyen du canaì antéricur; le test intérieur est en partic résorbé.

$3^{\circ}$ Dans le genre Mélanie, toutes les columelles sont obliques sous un angle de $10^{\circ}$, aree un tour de torsion aux deux tiers de leur hauteur, de sorte que le grand axe les coupe toutes en ce point.

Nous possédons des Clapes une coquille qui est conforme à la diagnose du Melania striala; elle est formée de 4 tours de spire, assez proches de l'extrémité postérieure.

Observant à la surface de ce fossile quelques fragments de test, nous arons présumé qu'il pourrait également s'en présenter dans l'intérieur et nous avons cherché à connaitre les dispositions des columelles.

Cette exploration nous a montré : $1^{0}$ que le test intérieur n'est pas résorbé, les cloisons possédant sensiblement la mêrne épaisseur que le reste du test et les ornements de la base s'étant conservés; $2^{\circ}$ les columelles sont verticales, par conséquent coupées sur toute leur hauteur par le grand axe; $3^{\circ}$ elles sont ereuses et démontrent par ce fait que la coquille était munie d'un ombilic ; $4^{\circ}$ leur forme est un cône régulier, tronqué au sommet, la base étant en arrière.

D'après cet exposé, on voit déjả que ce fossile né saurait ètre classé ni avec les Phasianelles, ni avec les Tones et encore bien moins avec les Mélanies.

Toutefois, pour nous assurer si l'échantillon que nous possédons des Clapes représente bien l'especce si abondante dans le calcaire à polypiers (Bajocien supérieur), 
nous avons !echerehé dans ce terrain des échantillons cassés, nous en avons mème cassé un grand nombre et nous sommes arrivés à ce résultat : un échantillon est muni de sa columelle, en forme de colonne légèrement conique, montrant l'intérieur rempli par de la roche ambiante; un antie ćchantillon privé de sa coh:melle montre un tube géodique, tapissé de cristaux de chaux carbonatée, ce qui démontre que la coquille était munie d'un omidie au moins dans sa partie interne.

II résulte de ees faits que Réchantillon des Clapes montre une constilution interne identique à celle que possècent les fossiles du calcaire à polypiers sousjacent au précédent.

En continuant ce mode dinvestigation pour savoir quelle est celle des coquilles qui possède une disposition analogne dans les columelles, nous arrivons d'abord au genre Nulice, dont la derniere columelle est iftentique it celle du fossile tus Clapes; elle est légèrement conique, tronquée au sommet et perpendiculaire it la base; mais lit précédente columelle est placée à gancire de la dernière et ne se troure plus dans la ligne du grand are; caractère sann retroure identigue dais les Turbo.

En second lieu, nous avons le geare Butime, doat le Bulimus decollatis montre des columelles verticales, plus grosses en arriere qu'en arant, ef toutes comprises dans le grand axe; mais la vartie antérieure n'est pas tronquéc et elle se continuc ave la columelle suirante; de plus, le test intérieur est en partie résorbś.

Entin, nous mentionnerons l'A gathine, qui a ses columelles placées suivant : grand axe; mais clles sont en forme de canal enroulé, dont un bord ra, ca sé tordant, rejoindre la columclle suivante pour se continuer jusqu'à l'extrénitú.

Yous pourrions cincore ciler les Riostellaires, les Chenopus, les Cônes, cte., quui tous présentent quelques rapports, mais jamais une identité arec les caracté... que nous avons exposés plus haut.

D'après l'ensemble de ces faits, nous sommes conduits à reconnaitre que le syetème que présentent les columelles n'appartient à aucungenre connu, et qu'en conséquence il y a lieu d'en créer un nouveau. Mais, bien que cette appréciation nous paraisse inédite, ne la tronrant indiquée par aucun atteur, nous devons cependant reconnaitre que notis arons élé dévancés pour le classement de ee fossile; M. Deshayes, guidé par la sureté de son couj d'wil, l'avait, depuis longtemps, rangé dans sa collection sous le nom de Bourgetic, en la dédiant su savant géologue M. Bourget.

Nous adopterons done ce nom pour spéceilier le Melania striata, Sow.

Préjugeant la forme de l'animal et la disposition de ses organes d'après la forme de l'ouverture et la disposition des columelles, nous dirons que ce nouveau genre devra trouver sa place assez proche des Natices, dont il présente une certaine similitude d'organisation interne, plutôt que près des Melania ou des Chemritzia, dont il s'éloigne complétement. 
Natica bajociensis, d'Orb.

D'Orbigny, Paléont. franç., t. II, p. 189, pl. 289, fig. 1-3.

Lès échantillons se rapportent exactement à la description et aux figures de d'Orbigny; dans l'un d'eux, la suture est plus oblique et marquée de deux courbures dirigées en sens inverse; ce fait est d'autant plus remarquable que les espéces de l'oolithe inférieure, figurées par d'Orbigny, se distinguent par une suture parfaitement rectiligne, et il faut remonter jusqu'au Corallien pour trouver des sutures douées de cette double courbure.

Localité : les Clapes, assez abondant.

Natica LoRieri, d'Orb.

D'Orbigny, Paléont. frunc., t. II, p. 190, pl. 289, fig. 6-7.

Localité : les Clapes, assez rare.

Natica ranvillexsis, d'Orb.

D’Orbigny, Paléont. franc., t. II, p. 193, pl. 290, f. 34.

Localité : Gravelotte (marnes), moules assez communs.

Natica pictaviensis, d'Orb.

D’Orbigny, Paléont. franc., t. II, p. 191, pl. 289, figg. 8-10.

Les échantillons que nous rapportons à cette espèce diffèrent un peu des figures données par d'Orbigny; leur taille est plus grande, tout en présentant le mène nombre de tours; ceux-ci sont plus renflés et plus arrondis; le dernier tour est couvert de plis irréguliers d'accroissement et de stries obsolètes, nombreuses, irrégulières, en ceintures, qui déterminent avec les premiers des croisillons qui couvrent toute la surface.

Ces ornements, qui ne sont pas mentionnés par d'Orbigny, sont identiques, ceux que présentent quelques Natices coralliennes: $N$. rumpellensis et $N$. grandis.

Localité : les Clapes, assez abondant.

Natica abducta, Phil.

Philipps. Ill. of. the Geolog. of York, p. 120, pl. 11, lig. 35. D'Orbigny, Paléont. frane., t. II, p. 189, pl. 289, fig. \& et 5.

Localité : Gravelotte (marnes), assez rare.

Natici Zetes, d'Orb.

D’Orb.; Paléont. frane., t. II, p. 197, pl. 291, fig. 7-9.

Localité : Angevillers (près Thionville), fort rare. 
Natica Zelisia, d'Orb.

D’Orbigny, Paléont. franc., p. 195, pl. 290, tig. 7-8.

Le moule se rapporte exactement aux figures données par d'Orbigny, et nous y avons constaté la présence d'un ombilic, caractère qui n’est pas mentionné dans la description de d'Orbigny.

Localité : Longwy (glacis), fort rare.

Nerita gea, d'Orb.

D’Orbigny, Paléont. franẹ., p. 232, pl. 302, tig. b’-7.

Localité: (iravelotte (calcaire jaune de Jaumont), assez commun.

Nerita pulli, Roem.

Homer, Ool., Geb., p. 153, pl. 9, lig. 30.

b'Orbigny, Paléont. franc., p. 236, pl. 303, fig. 4-6.

Cette petite coquille se rapporte exactement a la deseription et aux figures données par d'Orbigny, et nous la considérons comme identique, bien qu'elle soit indiquée pour le Corallien.

Localité : les Clapes, assez rare (Collection de Il. de Lambertye).

Patiodes sobosa, Morr. et Lye.

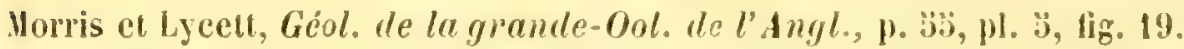

Notre échantillon se rapporte exactement à ha figure el à la description données par ces auteurs, bien qu'il présente une tailledlouble que la coquille figurée; ne possédant qu'un moule, nous ne saurions discuter sur l'exactitude de la détermination établie; mais, d'après la forme anguleuse de l'ouverture et la disposition du labre, nous pensons que cette espece trouverait mieux sa place avec les Troques.

Localité : Longwy (glacis), fort rare.

Trocius Acasta, d'Orb.

D'Orbigny, Paléont. frane., 1. 11, p. 274, pl. 312, lig. 13-13̈, Bathonien.

Localite : Ies Clapes, fort rare (Collection de. . de Lambertye).

Tnoches Acret, d'Orb.

D'Orbigny, Paliont. frane, 1. 11, 1. 27k, pl. 313, fig. 1-4.

D'Orbigny indique cette espèce pour le Bajocien, el, bien que nos échantillons s'y rapportent, ils possèdent en plus deux fines côtes sur le derniel tour.

Localité : les Clapes, assez rare (Collection de II. de Lambertye). 
Troches inornatus, Mu. sp.

Monadonta lavigata, Munster in Goldfuss, Petref.Germ., 1. III, p. 101, pl. 195, fig. 5. Trochus baldus, D'Orbigny, Prodrome, t. I, p. $33 \mathrm{u}^{\circ}, \mathrm{n}^{\circ} 103$.

Dimensions : hauteur, 8 mill.; largeur, 9 ; longueur relative du dernier tour, 400 0 0 . Cette espèce se rapporte exactement aux dessins et à la deseription données par foldfuss pour une coquille de l'Oolithe inféricure; d'Orbigny a établi dans son Prodrome un Trochus baldus, qu'il rend synonyme du Monodonta lcevigata, et qu'il place dans l'Oxfordien, malgré les indications contraires de Goldfuss; ne le connaissant pas dans la faune francaise, il ne l'a pas reproduit dans sa Paléontologic.

Localités : Ies Clapes, très-commun; Gravelotte, assez rare.

Trocius Zenobius, d'orb.

Dorbigny, Paleont. franc., t. II, p. 289, pl. 317, lig. 9-12, Bathonien.

Localité : les Clapes, fort rare (Collection de M. Colliez):

Trochus biarmatus, Mu.

Munster in Goldfuss, Petref. Germ., t. HI, p. 333 , pl. 180, fig. 2.

D'Orbigny, Paliont. frane., t. Il, p. 271, pl. 312, fig. 1-4.

Celte espèce, de 10 millim. de hauteur, a son dernier tour un peu renflé et se rapporte mieux à la figure donnće par Goldfuss qu'à celle de d'Orbigny ; celle-ci, formée comme les autres d'une spire à 6 tours, a 14 millim. de hauteur et montre un cône plus régulier.

Localité : les Clapes, assez commun.

Trochus angulatus, Mu.

Munster in Goldfuss, Petref. Germ., t. III, p. 56, pl. 180, fig. 7.

Turbo angulatus, d'Orbigny, Prodrome, t. I, p. 266, n 106 (Bajocien).

Nous n'avons pu saisir les motifs qui ont porté d'Orbigny à changer la classitication de Nunster, surtout quand nous voyons nos échantillons concorder avec les figures et la description établies par d'Orbigny pour le Trochus Mosa du Corallien (Paléont. franc., t. II, p. 299, pl. 320, fig. "ै-8); celui-ci ne diffère que par le labre un peu moins anguleux et par les stries longitudinales du dernier tour, ornement que peut-être la fossilisation n'a pas conservé dans les nôtres.

Localités : les Clapes, fort commun, Gravelotte (marnes), fort rare. 


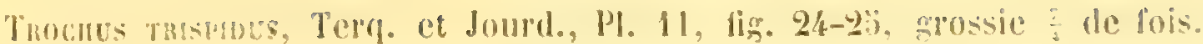

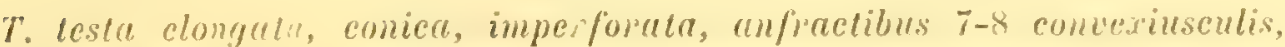
longitudinaliter costis tribus tuberculosis, una posteriore manstione, transver*is lamellis ad nodos decurrentibus ornatis, basi convexa, "̈-T cingulis nuberrulosis muxita, unertura ovali, columella spissa, impressa.

Dimensions : longneur 17 mill.; largeur. II mill, ; rapport dlu dernier tour 300 06. Coquille allongée, eonique, non ombiliquée, formée de $7-8$ tonrs, lígìrement convexes, ormés longitudinalement de trois côtes fubereuleuses, la postéricure beatucotip plus finc, et de lamelles nombreuses élevées, décurrentes et rejoignant les tubereules; ourerture ovale, botd columellaire cjais el muni d'un sillon longitudinal; base conrexe, ornée de cincl cotes lubereulenses en ceinture al de deux autres plus courtes pres de l'ourerture; sutures larges at profondes, marquées d'un cordon de petits nœuds.

Localité : les Clapies; assez commun.

Observation. - Dorbigny, dans la Paléontolagie francuise, al figuré, sous le nom de Purparimu, phusieurs fossiles, dont il n'a fait antene mention dans le texic.

II. Eudes-Deshongehamps, dans ses obserrations concernat quelques uastéropodes fossiles (1), a ctabli le senre Eucyche, appelé à eomprendre des fossiles rangés jusqu'ators dans les Tubo, les Trochus et les Purpurinu.

Ce nouveau genre a pour caractère la ténuité du test el principalement les ormements, comme son nom l'indique d'ailleurs: 1"un test très-minee; 2" des whis transversaux; '30 des plis longitudinaux souvent ondulés et bifuryués.

liespèce que nous figurons, bien que munie de semblables ornements, n'a pu itre rangée daus ce genene par ces motifs:

fo Le labre a la sérité est minee, mais le bord columellaire est fortement cneroûté et marqué d'un sillon qui indique que la coquille éhat munie dum vperenle épais : to la coupe du fossile montre un test épais sur ionte sa hauteur ; 30 les columelles sont placées comme dans les Troques: la 1 columelle interne est perpendiculaire it la base, la gave est en arrière, ì droite de la précédente el oblique à ganche; il en est de même de la $3^{*}$. M. Deslongehamps ajoute que ces coquilles, en rason de la ténuité de leur test, deraient êlre munies d'un opereulé corné, attendu que "si ces coquilles avaient été pourvues d'un opperrule calcaire, on aurait ecrtanement retrouvé eet opereule, qui aurail dit se tonserver en même temps que le test lui-même de la coquille; et jamais, dans les terrins furassiques anciens, nous navons trouvé d'operenles calcaires.

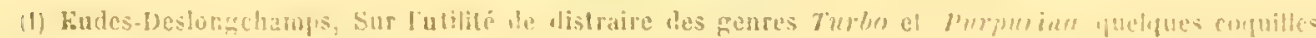

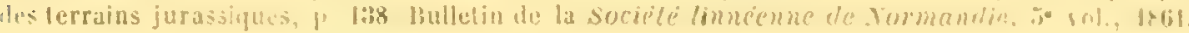

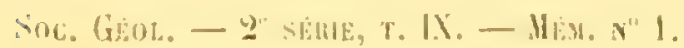


Nous ne croyons pas que feelte observation soit parfaitement juste : $1^{\circ}$ nous voyons, dans certains genres (les Natices, par exemple) les espèces avoir les unes un opyercule corné et les autres un opercule calcaire; $2^{\circ}$ dans certaines couches imperméables de Fontoy, nos recherches microscopiques nous on! donné de ecs opereules calcaires; 3 "la texture des opercules est différente de celle du test des coquilles, ce qui peut être démontré très-facilement; en mettant cnsemble dans de l'eau acidulée un opereule et une corquille, l'un sera dissous, quand l'autre sera à peine attaquée.

Trochus unteatus, Terq. el Jourd., Pl. II, fig. 26-27; grossic $\frac{\text { gr }}{2}$ de fois.

$T$. lesta conica, imperforata, anfractibus 6 planis, longitudinaliter octocostatis, costa anticu minima, simplici, aliis tuberculatis, velut texturatis, ultimo anfrache supra multicingillato, externe anguloso, apertura biangulata, columella unidentata, basi subplana, multicingillata, subearinata.

Dimensions : longueur 9 mill. ; largeur 7 mill. 5 ; rapport du dernier tour 50010. Coquille conique, un peu plus haute que large, non ombiliquéc, forméc de (i) tours plans, ornés de 8 côtes longitudinales, dont la postérieure et la $3^{\text {me }}$ antéricure plus grosses, et les deux antérieures très-fines, l'antérieure lisse, les intres tuberculeuses, comme texturées; base à peine convexe, ornée de fines stries concentriques, carénée sur son pourtour; ouverture bianguleuse extérieurement, calleuse sur le bord columellaire, munie d'un sillon longitudinal et d'une grosse dent.

Par sa forme conique et ses tours plans, cette espèce présente beaucoup de rapports avec le Trochus brutus, d'0rb. (Paléont. franę., 1.11, p. 383, pl. 315, f. 13-16. Bathonien); elle en diffère par te nombre de ses coites tuberculeuses ot frar les ornements de la base.

Localité : les Clapes; assez rare.

(Coll. de M. de Lambertye et de M. Colliez.)

Oxestus burtovensis, Lyc. I']. IV, lig. 11, 12, I3, grossi 2[3 de fois.

SIorris et Lycètt, Suppl. des Moll. de la yrande Oolithe de l'Angl., p. 103, pl. 45, lig. 7 et 7 a (du Forest-marble).

Notre espèce se rapporte exactement aux figures et à la description domnées par ces auteurs, sauf le large ombilic qui est indiqué et que nos échantillons ne présentent pas; on ne saurait le voir dans la large dépression qui occupe le centre et qui est couverte par une callosité.

Cette espèce diffère du Trochus heliacus, d'Orb. du toarcien (Paliont: frane, p. 269, pl. 311, f. 8-10) par sa base striée el ses tours moins obliques, bordés 


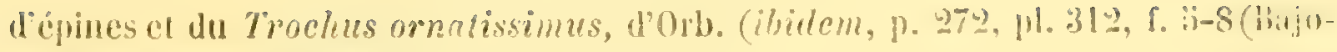
cien) par sa base concave.

Localité : les Clapes; fort rare.

(Collections de Hus. Colliez et de Lamberlye.)

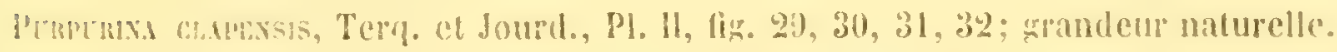

P. testa clongata, conica, imperforata, spira turriculalu, anfraclibus quinis, angulatis, longitulinatiter striis numerosis, regularibus at transversim 16 costis obtusis in dimidiu ultimo anfractu evanesrentibus nunatis, in postica purte nodulosis, strits carentibus, upertura ovali, antice patula, postice angustuk, labin tenue, columetla spissu, integre, arenate.

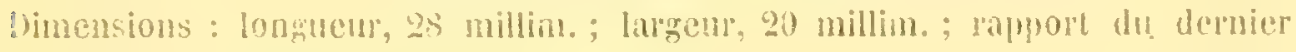
lour, 当 ol 0 .

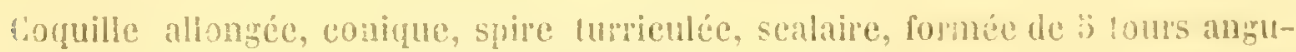
leux, ornés de nombreuses stries lougitudinales, rérnibeses de de 16 tubercules se prolongeant jusquu à la moilié du dernier tour: méplats oruís du prolongement des cotes lubereuleuses et privés de stries; ourepture ovale, élatée en avant et rétrécie en arriere; labre minee, tranchani, hord coltumellaire épais, arqué, base non ombiliquée, ornée de stries conecntriques.

Nous ne sarons si celte espece peut étre rapportée d̀ l'une de celles qui se trourent indiquées dais le Prodrame de d'orbigny $(t .1$, p. 270) et dont la description est trè-incomplète. H'me part, elles ne se trouvent pas daus sa Paléonrologie frunctise; dunc autre part, celles qui y sont figures ne sont pas derites dans le texte.

Localite: : les Clapes; assez commun.

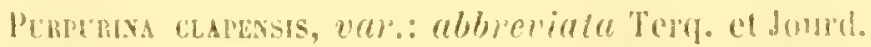

P'. II, lig. 28 el lig. 3\%. Lat figure $2 x$ grossic 2 fols.

Cette espece a les mèmes ornements que la précédente el en diflère par unc spire beaucoup plus cotirte, et dont le rapport entre l'ourerture ef lat longuer. tolale est de 75010 .

V. Piketty nous a communiqué une l'urpurine du grès supraliasique (montagne Pelée, prés de Thionville) qui présente tous les caractères de celle cispece el qui ne peut en être distinguée.

Loeabite : les Clanes; fort rare. (Coll. de M. de Lambertye.) 
Straparoles colosates, Terq. el Jourd., P. 1ll, lig. 4, 5, 6, grossie 2 fois.

S. testa compressa, spira abbreviata, anfractibus quinis, leniter scalaribus, planulatis, angulo 12 nodis elatis munito, latere prululum arcuato. basi angustissim?, unbilico mayno illue profur ulo, angulo octo nodis elatis instructo, apertura laterali, quadrilatera, angulis obtusis.

Dimensions : Jargeur, 9 mill.; hauteur, ij mill.

Coquille beaueouj) plus large (que haute, épaisse, spire déprimée, formée de 5 tours devenant successivement plus scalaires, les deux derniers tours onés de stries coneentriłues, obsolètes et munis sur l'angle de 12 nœuds élevés; pourtour légèrement convexe, base trés-étroite, munie d'un large ombilic, laissant it décourert les sutures internes, el bordé de 8 protubérences dentiformes, se reproduisant pres de la suture, mais moins grosses.

Cette espèce, rui a quelques rapports avec le $S$. allus, d'Orb. (Poléont. frane., 1. II, p. 314, pl. 332, fir. "3-8) par la forme de l'ouverture et par l'ensemble des ornements, en difière par la disposition sealaire des tours et par un plus grand nombre de protubérances.

Localite: : Ies Clapes; fort rare.

Solarium fothosum, Terd. et Jourd., PI. III, fig. 1-2-3; grossie 2 fois.

S. Lesta compressa, spirce abbreviata, anfractibus sex subscalaribus, planis, vel paululum cxcuvatis, engulo 14 nodis clatis et all suturam $22-24$ minoribus, idque 4 cingulis longitudinalibus ornatis, latere solundato, basi convexiuseulu, multicingulate, umbilico infundibuliformi, nothlis elongatis circumdul", apertura orbieulari.

Dimensions: largenr 7 mill. ì 9 mill. हैं ; hautcur \& mill. il

Coquille tris-déprimée, spire courte, beancoup pins large que haute, formée de (i tours très-légèrement scalarres, faiblement convexes en arrière, un peu exearés dans le milieu of arrondis sur le bord, ornés extérieurement de 14 gros nouds et de 22-24 plus petits près le la suture; pourtour arrondi, base légèrement convexe munie d'un ombilie étroit, jrofond, très-évasé et orné de nodosités allongées. traversees par de nombreuses stries eoneentiques, ouverture orbiculaire.

Cette conuille se distingue par ses tours un peu sealaires qui laissent à déconver les ornements de chaque tour, et par ses stries eoncentriques qui donnent à l'ensemble une grande élézance.

Localité : les Clapes; assez commun. 


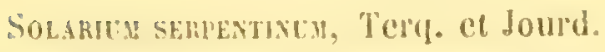

P'. III, fig. $7,8,9$; grossie 2 fois.

S. lestu nucleate, spira abbreviala, anfractibus 6-7 conjunctis, suturis vin ronspicuis, interne nodulis numerosis ad norlos externos decurrentibus, cingillis concentricis ornalis; latere rotundato, basi leniler impressa, cingillatu, clathratn, umbilien luto, canuliculato, infundibuliformi, nodulis elongutis instructo, apertura orbiculuri.

Bimensions : largeur 14 mili, ; hanteur 7 mill.

Coquille hémisphérque, deux fois plus large que haute, spire dépriméc, forméc de (i-z tours, remontant un peu sur le tour préédent, à sutures a peine indiquées. le dernicr lour un peu sealaire près de l'ouverture; tours ornés près de la suture d'une rangée de petits nouds s'allongeant sur le méplat pour se joindre à des nourls moins nombreux et plus gros du pourtour; còté artondi, bașe un peu dielive dans to milieu, ornée de plis d'autant plus nombreux et hus allongés quils approchent de louverure; ombilie large muni d'une dépression circulaire et recerant le prolongement des plis de ha base; ouverture onbiculare; toute ba surface converte de stries concentrigues déterminant des croisillons réruliers.

Localité : les Clapes; fort rare.

Tumo buvigieas, dorto.

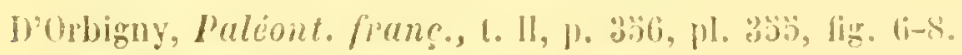

Localité : Longwy (glacis); fort rare.

Tunbo Ire.YTori, Goldi.

fioldiuss. Petref. Germ. t. :, 1, 9!, pl. 194. f. \$.

Localite : les Clapes; fort rare. (Collection de It. de Lamberlye.

Themo scancitts: Heb, et best.

Hébert el Lugène Ludes Deslongehamps, Vémoire sur leg fossiles de drontrenil - Belluy. p. 209, pl. 2, f. 10, a, b, e. Bubledin de la Soriété limnéenne de Normandie, le vol, 1867.

Cefte espèce du callovien est identique a nos fossiles des Clapes, d’abord par la description el les dessins, mis par la eomparaison des fossiles entre eux; les ornements sont les memes, et on y remarque le earactere principal: le demice tom est largement séparé de la spire, of la derniere sulure est très-profonde, dispositions analogues à celles (que possedent eertaines l)anphinules.

localite : les Clapes; assez commun. 
Terno puchelus, Terq. et Jourd. (1)

(Pl. 11!, fig. 10, 11, 12. Grossie 12 fois.)

T. testa minima, dopressa, spipa brevi, anfractibus is scalaribus, duobus primis lavigatis, mo transversim stricte striato, carinato, ultimo interue convexiusculo, noluloso, externe striato, circiler abciso, striato, carina utrinque limitato, basi convexa, cinfulu circumelta, strisque nodulosis radiantibus ornata, umbilico lato, npertura zolundata.

Dimensions : hauteur, 1 millim. $" 3$; diamètre, 2 millim.

Corquile dépriméc, phus large que thaute, à spire courte, formée de 4 tours scalaires, les deux premiers lisses? tet arrondis, te troisième légèrement renllé près de la suture, orué de nombreuses êt fines stries rayonnantes el linité par une carche; le dernier tour orné, sur sa parie convexe, de 18 à 20 lubercules alIongés, se terminant par trois ou quatre stries rayonnantes, pourtour limité des deux côtés par me carène ćlevée, tronųué verticalenent et ormé de fines strícs Iroitos ef trùs-serrées ; base convexe, "munie extéricurement d'un fort sillon et ornée de fines côtes rayonnantes, se continuant dans un ombilic larme, infundibuliforme, ouverture ronde, bord columellaire tris érasé.

Localite : les Clanes; fort rare.

\section{Tacosonom.}

Pour mémoire, nous indiguerors un moule indéternimable.

Localité : lonizwy (glacis).

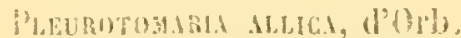

DOrbigny, Puléont. frume., t. 11, 1). 490, 11. 390, (Bajocien).

Tos échantillons diflerent un peu de la ligure de d'orbigny; is ont les tours Hus rentrants près de la suture, et la base est enticrement plane.

Localité : les clajes; fort rare. (Coll. (ic H. le ly: follicz.)

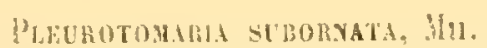

Nunster in Goldfuss, Petref. Germ., t. 111, p. 74, pl. 186, lig. :3.

Localité : Amanvillers (marnes sableuses); assez rare.

Puevrotoman mutabilis, Des!.

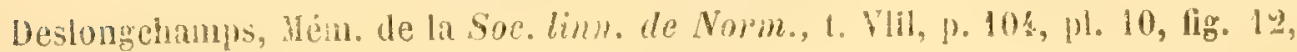
11. 11, fi $\div$. 1…

Deslongehamps fail observer (1). 103); (que celle espice est, sous plus d'un a apport, l'une des plus importantes du genre; e'est une des eoquilles caractéris-

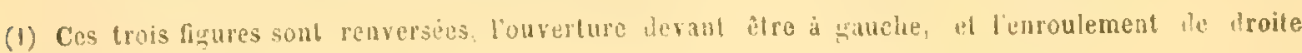
a gatche. 
tigues de l'oolihe ferrugincuse, puisqu'elle ne se rencontre ni an-dessus, ni andessous; la plupart des variétés étant fort communes, elle devient dun emploi commode pour reconnaitre l'oolithe ferrugineuse, dans le cas où les caractères minéralogifues de cete sous-formation ou ceux de superposition sont pen évidents. o

L'espece typique et deux de ses variétés se trourent dans plusicurs localités du Bathonien inférieur.

bocalité : Longwy 'glacis); assez commun (Collection de N. le docteur Colliez).

P'Lelrotohana meralils, dav. palula, Desl.

Deslongehamps, L. C., p. 111, pl. 10, fig. 12.

Localite : les Clapes; fort rare (Collection de W. le docteur Colliez).

Plevnotomana mitabis, ear. cireumsuleala, Desl.

Deslongchamps, L. C., p. 112, pl.11, tigr. 2.

Con Pleurotomaria circumsulcala, d'Orb., Paléont. frane, [1. 470, 11.381 , fige. (i-10.

Nos échantillons se rapportent exactement ì l'espece figurée par Deslongrchamps; les tours sont évidés près de la suturé, rendant ainsi le eordon des neends d'autant plus saillant. Dans les figures données par. dorbigny, les tours sont légèrement convexes et la coquille est régulièrement conique.

Localité : les Clapes; fort rare (Collection de M. le doeteur Colliez).

\section{Plecrotomatia.}

Yous mentionnerons, comme indéterminables, un moule plus que pugillaire, qui 11'a conservé aucune trace de test, et un fragment de coquille qui a appartenu a une espèce conique de grande taille; sa surface est ornce de nombreuses coifes en ceinture et d'une large bandelette placée près du bord inférieur.

Localités : les Clapes, Gravelotte (marnes); fort rare.

Plevrotomaria istermedia, Terq. el Jourd.

l'l. III, fig. 13-14, grandeur maturelle.

P. Lesta discoidea, spira depressa, circiter obluse carinata; apice aculo; an/ructibus 6 , plano-convexiusculis, primis stricte clathratis, aliis cingulatis, fascia sinus prominula, haud mocul margine sita, cingulata, separata; ultimo anfractu expanso; basi convexiuscula, multicingulala, anguste umbiticata; apertura iregulariter tranguluri, labro columellari incrassato.

Dimensions : hautenr 14 mill.; diametre 36 mill.

Coquille discuide, spire légèrement saillante, à sommet aigu, munic d'une

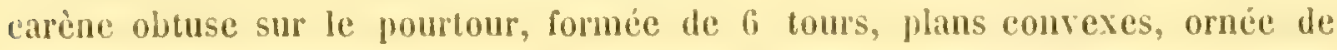
croisillons réguliers el serrés sur les premiers tours et sur le dernier de simples plis en ceinture; bandelette de l'entaille tres-saillante, à peu de distance du bord 
externe, dont clle est séparée par un sinus marué de deux stries; base subeonvere, ornée de nombreuses stries concentriques, plus serrées près des bords externe et interne „qu'an milien, munie d'un ombilic étroit, profond, légèremen rétréci par une mince callosité; onverture irrégulièrement triangulaire, columelle rerticale, épaisse.

Cette espece est intermédiaire entre le P. discus, Dest. (Deslongchamps, L. C., 1).98, pl. 16, fig. 3 (arviles de Dires), el le P. granulata, Goldf. (Deslongchamps, L. C., P. 98, pl. 16, fig. 4-\$), ou peut-être une variété de l'un ou de lautre, en tenam compte des olserrations qui suivent les descriptions de Deslongchamps.

Toutefois il nons a semblé convenable de représenter cette coquille, espèce on variété, pour indigner la forme qui se présente dans le Bathonien.

Localité: Fontoy; fort rare (Coll. de M. Piketty).

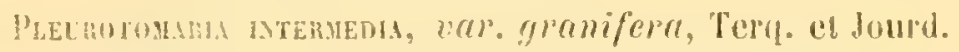

11. 11!, 4ig. 1\%, 16, 17, grandeur naturelle.

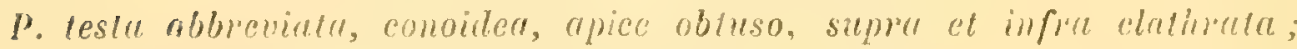
aperlura ovali, basi conivexinscula, unguste umbilicata.

Coquille courte, eonique, ohtuse au sommot, converte de croisillons sur ses denx surfaces, ouverture orale, base légèrement convexe, ombilie étroit.

Loealitis: Fontoy, les Clapes; fort rare (Coll. de N. Colliez).

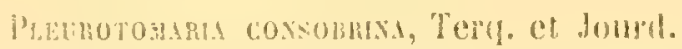

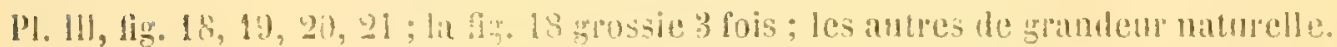

P. testu trochiformi, comica, apice obtusiusculo, anfractibus planis, postice wlicis mumerosis, palientibus omalis, fasciu simes promincule, angusta, in terlia anteriore parte sita, ullimo anfiactu ad basim obtuse anguluto, basi plana, au umbilicum plicatula, plivis radiamibus, obsoletis, mox evanescenlibus; umbilico latiusculo, parietibus plicato; apertura subquadrata.

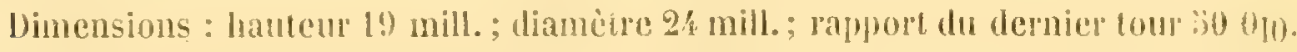

Corpuille trochiforme, conique, à sommet obtus, spire courte, forméc de tours planes, ornés en arriere de nombreur plis rayounants, sutures à peine risibles; entaille très-ćtroite, bandelette saillinte, placéc an tiers antérieur du tom, la: lemier angle obtus sur le pourtour; base plane, ornce de plis rayomant de l'ombilie, mais disparaissant bientôt; ombilie assez grand, strié sur ses farois, litssant apercevoir les tours internes, ouverture subyuadrangulaire, il lives minees.

Celle espèce se rapporle exactencut à la partie sugérienre du $\%$. Deshuyesii var. polyptica, Dest. Du lias supericur (Hém. de lu Soc. lim. de Nom., 1. IIII, 
p. \$31, pl. 9, fig. 6) et à la partie inférieure du P. Agathis, Desl. (Deslongchamps. L. C., , . $29, \mathrm{pl} .13$, fig. 8 ), de l'oolithe ferrugineuse; de la sorte nous avons di combiner la description de ces deux especes pour en constituer une troisième.

Loealité : les Clapes; fort rare.

Pueqrotomara Coldezi, Terq. et Jourd. P. IV, fig. 4, 5, 6.

P. testa turbinata, spira oblusa, scalariformi, apice obtusa, anfractibus sex convexiusculis, subtilissime striatis, confertim cingillatis, clathralis, ultimo anfraclu circiter obtuse carinato ; fascia sinus in medio anfractu sita, tricingillata; basi plana, anguste porforatr, multicingulata, rpertura rhomboidali.

Dimensions: Hauteur 16 mill.; diamètre 19 mill. ; rapport du dernier tour 40010 . Coquille courte, turbinće, ì sommet oblus, spirc légèrement scalaire, formée de b tours convexes, ornés de plis rayonnants, très-serrés, et de fines côtes longitudimales; bandelette de l'entaille grande, placée au milieu du tour et ornéc de 3 côtes très-fines; base plane, munie d'un étroil ombilic et courerte de croísillons très-serrés; ouverture rhomb idale.

Cette espece, par ses tours arrondis et par ses ornements, se rapproche du P. djax, llorb. (Paléont. Franc., P. 484, M. 388, fig. 1-3, Bajocien), et elle s'en éloigne par sa large bandelette et par sa base aplatie.

Localité : les Clapes; fort rare (Coll. de H. te Dr Colliez).

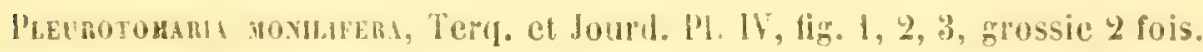

P. Lesta compressu, discoidea, spira planulutu, apice oblusa, anfractibus 4 planis, costis numerosis, areuatis, radiantibus ornata, ultimo producto, carina granulos't cireumdato: fascia sinus angusta, prominula, haud procul margine silu, sinu bicingulato, separato, basi subconvexa, margine depressa, radiatim. striete costulata, umbilico in/undibuliformi minuta, apertura subquadrangulari, columelta brovi, spissa.

Dimensions: Ilauteur 6 mill. : diamcitre 18 mill.

Coquille aplatie, à sommet oblus, spire discoüdale, formée de la tours plans, ornés de nombreuses côtes rayonamies, arquées; dernier tour bordé d'une carène ornée d'une séric de petites perles; bandelette étroite élevée, placéc près du bord externe, dont elle est séparéc par un sillon et ornée de deux stries longitudinales; base légèrement convexe, déprimée sur le bord, couverte de larges plis rayonnants; ombilic étroit, en forme d'entonnoir, laissant voir les tours précédents; ouverture subquadrangulaire, columelle courte et épaisse.

Cette espéce présente des rapports de forme avec le $P$. montreuilensis, lléb. et Desl. (Bull. de la Soc.limn. de Form., Fo volume 18:59-60. llébert et Destongrchamps, fossiles de Ilontrenil. - Lellay, p. 220, $\mu .3$, fig. 3.); clle en diflère

Soc. GBot. - SEALi, T. IX. - MrH. X" 1. 
par les tours de la spire beaucoup flus aplatis et par labsence de stries concentriques. Peut-ètre n'en avons-nous qu'une variété?

Localité : Ies Clapes; fort rare.

Alahia livigata, Jort. el Lye.

Morris el Lyeett. Foss. de la grande-oolithe de l'Angl., ire part., p. 17, pl. 3, fig. 3 ct 3 a ; Piette, Paléont. franc., p. 69, pl. 7 , fis. 4-10, pl.10, fig. 7-9 et pl. 12, fig. 6-9.

Localité : Gravelotte (marnes); fort rare.

Alama bicakisata, Mu. Sp.

Rostellariabicurinulu. hunster in Gohlfuss. Pebref.Germ., T. 11, p. 16, pl. 170, fig. 1 .

Localité : (ravelolte (marnes); fort rare.

Alafia multistriata, Piet.

Pielte, Bull. de la soe. Géol. di: France, T. Xill, p. 93, pl, 3, fig. 1-3. Piette, Paléont. fiane., pl. 4, fig. 4-5, pl.6, fig. 8-10.

Localité : les Clapes; issez rare.

Alaria gothica, Piet., H. 1V, lig. 16, 17, grossie 3al2 fois.

Pielte, Bull. de la Soe. Géol. de France, T. XII, p. 9\%̈, pl. 3, fig. \& et S. Piette, Paléont. frane., p. 33, pl.8, fig. 1-3, pl. 12, fig. 13-17.

Pour cette espèce nous possédons la coquille presque complète, munie de son aile; elle est rhomboïdale à angles arrondis.

Localité : les Clapes; assez commun, fort rare entière. (Coll. de N. de Lambertye.

Alaria Hames, Desl. Sp.

Roslellaria hamus. Eudes Deslongehamps, Mém. de la Sac. linn. de Norm., T. 1I, p. 173; pl. 9, lig. อ̈3-36. Plerocera hamus, d'0rb. d'orbigny, Prodrome, T. 1, p. 270 (non mentionné dans la Paléont. frane. ). Alaria hamus, E. Desl. Piette, Suppl. de la Paléont. franc., p. 39, pl. 3, fig. 10, pl. है, fig. 1 à 1 , ji. 6 , tiv. 18 et is.

Localité : les Clapes; asse\% rare.

Alaria trafidi, Bean, Phil. Sp.

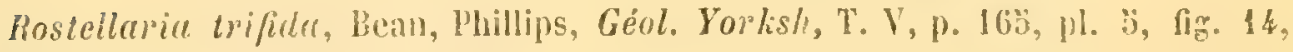
(Oxford-Clay), Rostelletrin trifida, Lud. Deslongchamps, Jém. de la Soc. linn. de Norm., T. Wh, 1. 171, pl. 9, fig. 27 ì 31. Plerocera trifida, d'orbigny, Prodrome, T. 1, 1. 357, n' 150. Alaria Lorieri, d'Orib. Pietle, Suppl. de ta 
paléont. frane.., p. 32, pl. 2, fig. 12-14, pl. 3, fig. 11-14, pl. 4, fig. 1-3, pl. 1, fig. $2-7$.

Deslongehamps fait observer qu'il a trouvé cette espèce, avec des tailles différentes, dans le lias supérieur, le calcaire ferrugineux, la grande-oolithe, Ji picre blanche, l'argile de Dives et celle de Hontleur,

Si cette observation est confirmée et s'il reste démontré que cette espèce, passant a travers tous ces terrains, a bien conservé tous ses earactères typiques de forme ct d'ornement, le fait serait fort remarquable et même unique, attendu qu'on ne connait encore aucun fossile du lias, remontant ainsi à travers les terrains oolithiques pour se produire identique jusque dans l'oxfordien.

$\therefore$ Localite : les Clapes ; fort rare.

Alaria clathatra, Terg. et Jourd., PI. IV, fig. 7-8, grossie :je fois.

A. testa clongata, conica, spira turriculata, anfractibus 9 in medio excavalis, utringue tricingulutis, angulo subncuto, lineis transversalibus, clathratis ornatis, suluris vix perspicuis, basi rotundatu, quatuor costis sparsis et ad columellam quatuor aliis minoribus strictis munitu.

Dimensions: longueur 13 mill.; largeur 6 mill. ; rapport du dernier tour 83010 . Coquille allongée, conique, spire turriculée, formée de 9 tours excarés dans le milieu, subaigus sur l'angle, ornés de chaque côté de trois lines côtes en ceinture et de nombreuses stries transversales, formant des croisillons; sutures a peine visibles; base arrondie, ornce extéricurement de quatre grosses côtes espacées et près de la columelle de quatre autres plus fines el serrées.

Cette espece se distingue de 1'A. mullistriata par la forme des lours phtus excavés et par l'angle plus saillant et plus aigu; les côtes longitudinales sont moins nombreuses et moins régulièrement espacées.

Localité : les Clapes; fort rare.

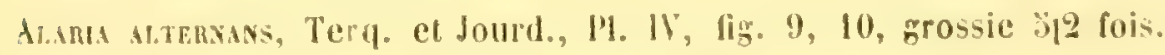

A. testu elongata, turriculata, spira fusiformi, anfractibus y planis, regularibus, nodis elatis, in anfructibus alternantibus et longitudinaliter is costulis ornatis, ultimo antice producto, acuminato, suturis profundis.

Dimensions: longueur 12 mill.; largeve 3 mill.; rapport du dernicr tour 60010 .

Coquille allongée, turriculée, spire fusiforme, formée de 9 tours plans, réguliers ornés de gros nouds alternant avec ceux du tour précédent et de "; fines côtes longitudinales, dernier tour terminé par un canal allongé; sutures profondes, base munie de stries concentriques.

Cette coquille, qui ne parât pas avoir atequis tout son déreloppement et dont le 
labre est cassé, ne présente pas les caractères génériques propres à l'oúverture des Alaria; son canal antérieur porterait plutôt à la classer parmi les Fuscaux.

L'échantillon étant unique et nous ayant élé comtiuniqué, il n’a pu être travaillé pour la recherche des columelles internes, qui auraient pu donner avec quelque certitude les caractères du genre auquel il faudrait définitivement le rapporter.

Localité : les Clapes ; fort rare (Coll. de I. de Lambertye).

Cenithum gexeyalense, Terq. et Jourd, P. IV, fig. 14, grossie 7 fois.

C. testa minima, conica, spira elongata, anfractibus brotundatis, costis elatis, obtusis, interstitiis minoribus ornatis, suturis profundis, basi rotundata, lavigata, apertura semi-lunari; breve canaliculata.

Dimensions : Iongueur 2,7 mill.; largeur 1, 4; rapport du dernier tour 60010 .

Coquille trìs-petite, conique, à spire allongée, formée de "z tours arrondis, ormés de cótes élevées, obtuses, plus étroites que les intervalles, sutures profondes, base arrondic et lisse, ouverture semi-lunaire, canal très-court.

Localité : Gravelotte, calcaire de Jaumont; assez commun.

Ceritimbs genicilatum, Terq. el Jourd., PI. IV, fig. 13, grossie 7 fois.

C. testa minima, conica, spira producta, anfractibus 6, quorum quatuor primis conjunctis, planis, costulatis, duobus ultimis goniculatis, costulisplicatis.

Dimensions: Iongeur 4,3 mill.; largeur $1,3 \mathrm{~b}$ mill.; rapport du dernier tour 250 0 0 .

Coquille très-petite, conique, à spire allongée et formée de 6 tours, les quatre premiers plans, ornés de còtes droites, les deux derniers genouillés aux $2 \mid 3$ de leur hauteur et mmis de côtes angulenses; ouverture ovale, canal antérieur très-court.

Le caleaire jaune de Gratelotte, identique a celui de Jaumont, ne renferme que des coquilles microscopiques ou des débris complètement brisés et indéter minables.

Ce Corithium avec le précédent est accompagné de quelques autres fossiles de même taille, Turbo et Trochus à l'état embryonnaire, Nerita gea, Asturte pisolina, Ostrea acuminalu.

Loealité : Crarelotte, calcaire de daumont; assez commun.

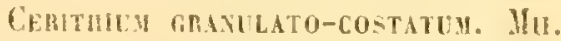

Munster in Golduss, Pelref. Gemm. T. 111, 1. 32, pl. 173, fig. 10.

Fous réunissons à cette espice quelques variétés qui constatent que les steries

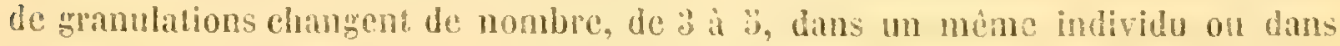
des individus differents; les granuhations elles-mèmes changent de fornte ou se groupent de façons difrirentes, sans que ees modifieations paraissent altérer le 
type; cependant une des rariétés, à bangées le tubereules, se présente avee une spire phus courte et plus renflée et la base plus arrondie, earactères qui donnen i la coquille une forme plus conique.

M.M. Ilébert et Eug. Eudes-Deshongchamps ont public (1) les fossiles de Montreuil-Bellay (Oxfordien inféricur) et ont représenté plusieurs Cérithes pami lesquels se trouse le Cerithium granulato-costalum, pl. VIl, fig. 1, illentique à

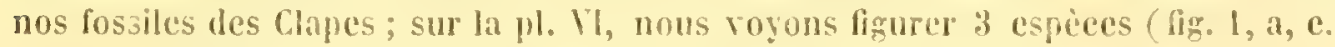
C. torlile, II. D.; fig. 2, a, b. C. Lorien, H. D; fig. I, a, b, c, C. fusiforme, II. D.) qui se rapportent à 3 sortes de nos fossiles que nous n'avons considérées que comme des variétés du-type, en raison des passages de formes et d'ormements que nous avions sous les yeux.

Par les divers envois qui nous ont été fuits, il nous a été permis de comparer tes fossiles de hontreuil-Bellay aux nôtres et de nous convainere do leur parfaite identité.

Localité : les Clapes; très-eommun.

)ENTMIUM XTEYS, SOW.

Sowerby, Jin. Conch., p. 108, pl. 70, fig. 12.

Xous rapportons à celte espece une cocquille qui ne nous a présenté aucune trace d'ormements.

Localite : les Clapes; assez commun, mais toujour's fragmentaire.

$$
\text { Cititol. }
$$

Nous arous trouré une pièce entière et quelques débris dans une conche de Fontoy et dans les mèmes conditions que celles que nous avons observées pour le lias: il fatut que les marnes soient exeeptionnellement imprégnées de sulfure de fer pour que cette coquilte ne soit pas détruite par les courants acidules.

Le bathonien inférieur donne ainsi une nouvelle station pour ce gente, dont lat présence n'a encore été signalée que dans le lias moven comme intermédiaire entre le terrain carbonifere ef le tertaire.

La piece d'Oscabrion est une médiane, tris-petite (hauteur el dianedre 2 mill.), ornée en arant de stries vertieales granuleuses of en arrière de stries obliques: la protubérance d'atlache est très-saillante.

Localité : Fontoy, \&" eouche; fort rare.

$$
\text { EMaghuna schars, sow. }
$$

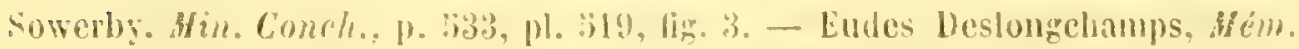
de la Soe. lim. de Norm., 1. 1H, p. 12:5, pl. 7, lig. 30-32.

Xous rapportons a celte espece unc counille tres-fragile que nous arons trouréo altachée sur une hime; le coté est ormé de grosses côtes altermant atee

(1) Bulletin de la socicle linnène de lormandie, 5. 101. 1861. 
de pettes, et dont les trois premieres séries sont munies sur l'angle de protubérances épineuses: les stries iransverses sont très-étroites, régulières et déterminem dans les intervalles des mailles carrées, profondes et d'une grande régularité.

¿ocalite les liapes ; fork rare.

\section{['atella Tessos, Eud. Desl.}

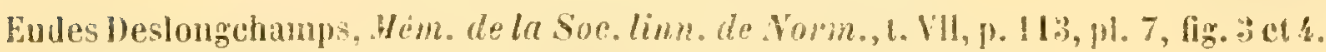
Lotalité : Gravelotte (marnes); fort rare.

\section{ICEPIIALES}

Gastrochemi meosmata, Des.

E. Deslongchamps, Hem. de la Soc linn. de Norm., t. VI, p. 226, pl. 9, fig. 19 à 29. Nous ne possédons de celte equille que la partie postérieure, qui montre d'une inanière forl nette les deux carènes, la ventrate plus saillante que la dorsale.

luocalité : les Clapes, dans un Isustrea ; fort rare.

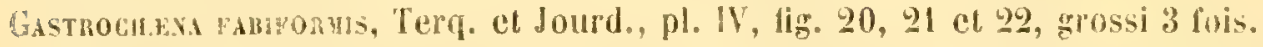

- Ǵ. testa elongata, fubiformi, transversim rotundata, stricte concentricis plicis undulosis orantu, antice acuminala, apertura cordiformi, postire strecuata, hiantula.

Dimensions: longucu 12 mill.; largenr 7 mill.; longueur buceale 2 mill.; longueur anale $10 \mathrm{mill}$.

Coquille allongée, fabiforine, arrondie transversalement, mune d'une échanerure cordiforme en avant et d'un fabble baillentent en arrière; bord cardinal antérieur droit et relevé, crochets trés-petits et arrondis; test très-mince ct fragile, orné de stries très-fines régulières et repliées suivant les contours de l'excavation antérieure.

Cette espèce diffère du G. subtrigona, Desl., Jém. de la Soc. linn. de Norm., t. VI, p. 223 , pl. 9, fig. 12, 14, 16) ei du G. lacryma, Desl. (ibidem p. 223. 14. 9, fir. 9, 10, 17), par son excavation beaucour moins profonde et par sou côté rentral presque vertical.

Localité : les Clapes; dans un Istestrea; assco comnun, mais fort rare entier.

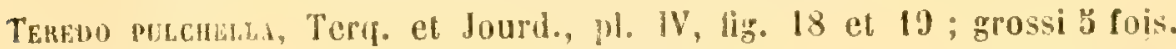

T. testu minimu, subglobosu, tenue, fragili, antice striis concentricis, subtifissimis, regularibus ormata, postice sulco plicisque obliquis instructo, antice

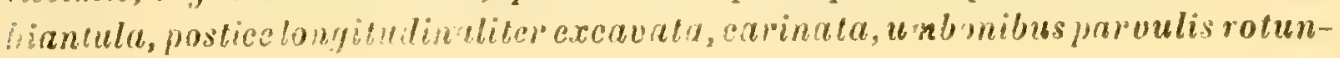
dertis.

bimensions : longueur et diametre so mill.

Coquille très-petite, subsphérique, à test mince et fragile, ornée cn avant de

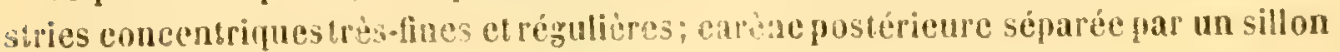


vertical et ornée de gios plis faisant un angle aigu avec les stries antéricures; munie postérieurement d'une excavation en forme de fente, baillante en avant, élargie dans le milicu et bordée par une mince arde, crochets tris-petits, arrondis et replicis en dedans.

Nayant pas troure de loge avee prolongement pour aueun des trois fehantillons que nous possedons, nous avons cependint the rapporter ce fossite au genre tareq antant par sa forme que par la disposition de ses ornements.

Localité : les Clapes, dans un lsqst!ece, arec les atres coquilles perforantes; fort rare.

\section{Gramc Phomaboma (1), Sow.}

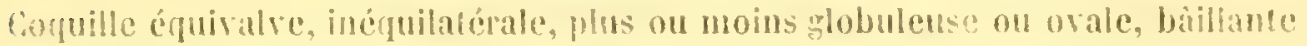
en arriere; test tres-lanelleux, orne de cotes rayonnantes, qui se reprodusent su

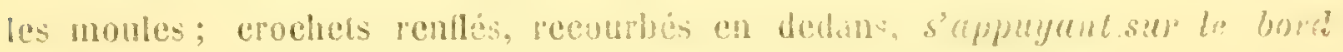

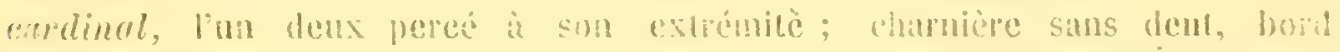

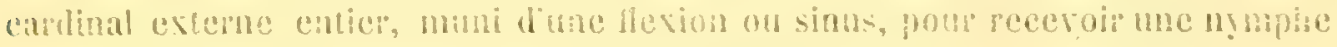
chaisse el courte; hond cadinal intene renthe arrondi et sintieux; corselet en

(1) H. Agassiz, dans son mémoire sur les Mraires fossiles (Agassiz, Études critiques sur les. Uhtlusques fossiles; Monographie des IIyes. Neuchattel, 1812-40.) a créé un grand nombre de genres fondés uniquement sur les caractères fournis par des inoules.

DOOrbigny (Prodrome) n'a maintenu aucun de ces genres, a négligé de mentionner les uns et a diversement classé les autres; ainsi les Pleuromyes et les Myopsis rentrent dans le genre Panopée, et les Gresslyes arec les Céromyes dans le genre Lyonsia.

1. Deshayes a examiné la nombreuse série de fossiles, qui, ne sétant présentée, jusquà cet jour, qu'ì l'état de moules, se montre avec les caractères suirants: " privée de dénts à la charnière, douée d'un sinus palléal et d'un biillement postéricur (Deshayes, Traité élémentaire de Coachyliologie, art. I'holadomye. T. I, p. 1/47). En conséquence il a reporté les genres d'Agassiz aux Pholadomyes, sauf les Gresslyes et les Céromyes qui sont maintenus quoique réunis dans un seul genre, et se trouvent compris dans la famille des Glycimérides.

Vhais dès l'instant qu'on peut étudier ces fossiles munis de leur test, on trouve que cerlaines dirisions étallies par Agassiz, bien qu'il nent lui-mème que des moules à sa disposition, acruièrent une valeur rielle. Alors aussi on est conduit à reconnaitre que, dans celte classifica fion, Agassiz a fait preure d'une sorte de préscience, qui lui a servi de guide. Ln elfet quelques-uns de ses genres, pour acqquérir une place stable et définitive dans la nomenclature, ne demandaient que la démonstration rationnelle des caractères génériques, que l'étude de la coquille pouvait seule fournir.

Cette étude est venue démontrer qu aucun des systèmes de classification, présentés jusqu’à ce jour par les différents auteurs qui ont eu à mentionner ces fossiles, ne peut leur ètre appliqué (1).

Nous pensons que l'opinion de $\mathbf{M}$. Deshayes, pour itre rendue parfaitement juste, demande à receroir l'adjonction de ces mots : munie d'un ligument simple; car il devient évident qque, dès

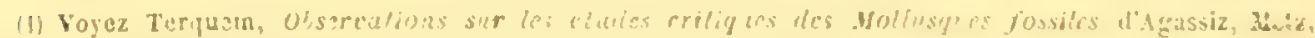

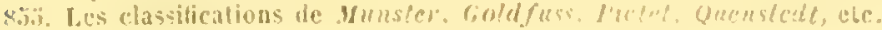


gouthiere, le bord d'une valve ne chevauchant pas sur celui de la valve opposée; impressions musculaires arrondies, sinus palléal formé de deux lignes uroiles, déterminant un angle peu ouvert et une très-courte languette.

Nous devons faire remarquer que pour les Pholadonyes et les llomomyes on trouve constamment les fossiles avec les deux valves parfaitement en place, bien que la charnière soit privée de dents el que les valves ne soient que juxtaposées; cette circonstance est due à ce que l'extrémité d'un des crochets est largement excavée pour recevoir profondément l'extrémité du crochet opposé.

Proladomy Murchisox, Sow., P. V, fig. 1.

Sowerby, Nineral Conchology, 1. 562, pl. 297, dig. 4. Agassiz, Monographie des Pholadomyes, p. 79, pl. 4 e, lig. :̈-7. Goldfuss, Petrefacta Germanire, 1. 11,

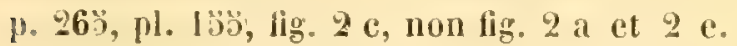

Ceite espèce, très-abondante et très-répandue, se montre partout identique el présente peu de modifications dans sa forme; clle est bien ylus rarement écrasée d'anière en avant que les autres espèces.

Partout à l'état de moule, cette espèce nous a donné, exceptionnellement pour les Clapes, deux échantillons munis de leur test, dont un a pu être fendu longitudinalement el nous a domné les dispositions de la charnière; ces cireonstances nous permettent d'ajonter à la diagnose du genre queífues caractères particuliers, que nous ne trouvons mentionnés par aucun auieur. Test généralement spathique, épais, foliacé, formé de conches superposces (nous en avons pu compter jusqu'a (6), s'exfoliant sans que ia surface perde rien de sa netleté. Ornements formés de còtes rayonnantes obtuses, noduleuses, traversées par des plis d'acroissement peu profonds. Crochets très-repliés en dedans, l'un ou parfois tous les deux pereús à leur extrémité. Bcillement postéricur, en fente verticale.

que des coquilles possident a la fois des nymphes et des cuillerons, elles doivent sortir de la famille des Glycimérides et quitter le voisinage des Pholadomyes, pour entrer dans celle de Osteademes et se rapprocher des Thracies.

Il résulte de l'ensemble de nos observations que le genre Goniomya vient seul se confondre avec les Pholadomyes; le genre IIomomya, tout en restant dans la famille des Glycimérides, est maintenu et se trouve rangé après les Pholadomves; le genre Hlatymya et une partie du genre Arcomya sont id véritables Psammobia; les genres Arcomya, Pleuromya (qui comprend les Myopsis) et. Gres lya (auquel se joint le geare Ceromya) rentrent daus la famille des Ostéodèsmes.

Daprès cet exposé, ii est à regretter que M. Agassiz ayant consiléré tous ces fossiles comme appartenant ì une seule famille, ait appliqué à ses genres des noms qu'il a fait dériver du genre Mya; il a ainsi consacré des erreurs, en rapprochant d'ume type unique, des fossile, dont plusieurs séries s'éloignent complètement. 
Corselet peu sensible et indiqué par un simple pli du bord cardinal, ordinairement plus prononcé el plus large sur la valve droite que sur la gauche; d'où il semble résulter que lime des valves, par suite du bâillement ventral, glisse sur la partic postéricure de l'autre.

Wymphe épaisse, courte, arrondie du côté interne, relevée du côté externe. oì elle détermine un canal étroit, dans lequel entre le ligament.

Charnière sans dent, ì bord eardinal entier, présentant sous les crochets un pli ou concavité, dans lapuelle s'insére la nymphe.

Impression palléale occupant un peu moins que la moitié de la longueur de la coquille et formée de trois parties distinctes: la supérieure horizontale, la moyonne régulierement arquée, l'inférieure très-oblique, ne formant pas de languette aree l'impression du bord.

Impressions musculaires superficielles.

Bäillemeut postérieur, en fente verticale.

Localités: les Chapres, firavelutte (mames), Longw! (glacis), fontoy, llayange, Gorze (eimetiere), la còte de Jay (vallée de Mance); partout abondant.

Pllolaboura texmbata, Terq. et Jourd., pl. Y, fig. $7,8,9,10$.

j. nucleo obovuto, supre abinflato, circitrir et postice compresso, umbonibus inflexis, subanticis, ore cardinali recto, costis radiantibus 6 , prima rugosa, rotundaln, duabns subacutis, anguslis, aliis vix conspicuis, costulisque concentricis, subregularibus, strictis, velui texturatis exornata.

Dimensions : longueur 70 millim.; hauteur 60 millim. ; épaisseur 33 millim. ; longueur buccale s’3 millim.; longueur anale :3is millim.

Houle obovale, renllé pres des crochets, comprimé sur tout son pourtour, crochets intléchis, subantéricurs; bord cardinal droil ; orné de 6 côtes rayonnantes, la première élevéc, arrondie, rugueuse, les deux suirantes très-étroites, subaiguës, les autres à peine visibles; convert, sur toute sa surface, de fines cỏtes concentriques régulicres, parfois onduleuses, qui donnent au fossile un aspect comme texturé.

Un échantillon a montré sur sa partie postérieure des côtes très-fines, presque horizontales, déterminant des croisillons aree les cütes concentriques.

Yous ne croyons pas que l'état comprimé des moules soit le résultat de da fossilisation, altendu gue tous nus bchantilons présentent la mème disposition.

Cette espece, par la disposition de ses ornements, se distingue de toutes les nutres.

Localiés: Gravelolle, (iorze (cimelièe); asse\% commun.

SOC. GEOL. - SELE, T. II. - VIYY. I. 
Pholadomya 'lietenit, Ag.

Agassiz. Éludes crit. sur les Holl. foss, Hon. des Phol., p. 34, pl. 3, fig. 13-13.

Cette espèce se distingue par une alternance de petites et de grandes côtes sur la partic antérieure; elle se trouve abondamment dans le calcaire ferrugineux avec une très-grande taille; dans le bathonien inférieur, elle est très-petite et fort rare.

Localité : les Clapes; fort rare.

Pholanomy costellata, Ag.

Igassiz. L. C., 1). 55, pl. 3, líg. 1-3.

Localités: Gorze (le cimetière), Longwy (glacis); fort rare.

Pholadomy Nyphacea, Ag.

Agassiz. L. C., p. 71, pl. है a, tig. 1-ว.

Localite : Longwy (glacis); fort rare.

Pholadomy beccardiug, A:.

Agassiz. L. C., p. 77, pl. "5, fig. 3-7 et. pl. 5 a , fig. 8 .

Localités: Gorze (cimetière), Longwy (glacis), Hayange (les hauteurs près de Ranguevaux); partout fort rare.

Pholadomya oveless, Ag.

Agassiz. L. C., p. 119, pl. 3, fig. 7-9,

et pl. 3-6, fig. 1-6.

Localité : les Clapes; assez rare.

Pholadomya proboscidea, Ag. Sp.

Goniomya (1) proboscidea, Ag. Agassiz. Études crit. sur les Hyaires, p. 17.

pl. 1, fig. 6-7; pl. 1 c, fig. 1-9.

Cette espèce est assez abondante dans le Bajocien inférieur et le supérieur:

Localités : Gravelotte (marnes), les Clapes; fort rare.

Pholadomya V.-Scripta, Sow. Sp.

Yya V.-Scripta, Sow. Sowerby, Mineral Conchology, p. 273, pl. 224, lig. 2-\$3.

Celte espèce se trouve assez fréquemment dans le Bajocien inférieur et mumie de son test, qui se montre orné de très-fines côtes rayonnantes granuleuses.

Localité : les Clapes; fort rare. (Collection de M. de Lambertye).

(1) La diayose du genre Coniomya, Ag. (Lysianassa, Mu. in Goldfuss, 1. 11, p. 261), fondée sur un see! caractère, do cöles en chevrons, nous parait loin dêtre bien assise. Le bord cardinal, que nous arow* pu complétcrent dégager, est droit, presque linéaire, entier sur toute sa longueur et moatre aingi lea caractères que nous arons reconnus aux pholadomyes. 
Gente llomomy, dgassi\%. I'l. $V$, lig. 11 et 12, grandeur naturelle.

Yous ne reproduirons pas la discussion qui concerne ce genre et qui est longuement exposée dans nos observations sur les études eritiques de H. Agassiz; nous nous attacherons à préciser les caractères de la coquille et de sa charnière.

Coquille équivalve, allongée, très-inéquilatérale, très-courte en avant, bâillante en arrière; parfois ornée de côtes rayonnantes superficielles dans le jeune âge; à lest foliacé, simplement marqué de plis d'accroissement; crochets rentlés, recourbés en dedans, ne s'appuyant pas sur le bord eardinal, l'un des deux peré ì son extrémité; charnière sans dent; bord cardinal très-mince, foliacé, Ie bord antérieur se continuant sous les crochets sans inflexion jusqu'au point de l'inserlion de la nymphe et où se produit une étroite échancrure, élargie en dedans; bord cardinal interne rentlé, arrondi et sinueux; nymphes courtes, subtriangulaires, aiguës près des crochets, phtis larges en arrière et séparées du bord cardinal par un profond sillon, ligament count, épais, résistant.

Sous mettrons en regard les caracteres principaux qui font distinguer les llomomyes des Pholadomyes:

Pholimines.

Bord cardinal entier, renllé en forme de boudin.

Nymphes placées dians une dépression, entièrement soudées au borl cardinal.

Crochets appuyés sur le bord cardinal.

Côtes plus ou moins grosses, layonnantes et à tous les âges.

\section{HoHomyes.}

Burd cardinal incisé sous les crochets, en lame très-mince.

Vymplies placées sur lo bord cardinal, détachées par un sillon en arriere.

Cirochets non appuyés sur le bord carlinal.

Còtes rayonnantes superticielles et dans le jeune âge seulement.

Honomit gibessa, Sow. Si).

Mactra gibbosa, Sowerby. Min. Conch., p. 70, pl. 42; Ilomomya gibbosu, Agassiz. Mon. des Hyaires, p. 160, pl. 18. Pholadomyn gibbosa, d'0rbigny, Prodrome, t. 1, p. $274, \mathrm{n}^{\circ} 233$ (1).

Cette espèce a 6 ou 7 cóles rayonnantes très-fines dans le jeune âge ; elles a'atteignent pas te milieu de la eoquille dans ladulte.

Localités: les Clapes, Gravelotte; abondant.

Homorya Vezelim, Laj.

Celte espèce se distingue du $1 /$. gibbosa, par le bord supérieur el l'inférieur sensiblement parallèles; elle est privée de côtes dans le jeune tૈge.

Localités: Gravelotte (marnes), Longwy (glacis), Fontoy, Gorze, Conflans, ete.; partout assez abondant.

(1) Par une faute typographique les numéros 233 a 238 magquent dans le brodrome, a la page 274

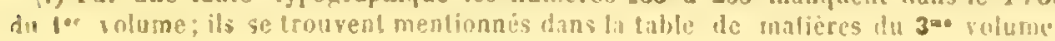


Gexhe Psamoda, Lamarck.

Nous avons pour.l'étude de ce genre tous les éléments nécessaires, et même en abondance, des coquilles parfaitement conservées, des valves isolées et vides, ainsi que des moules complets, qui se rapportent d'une manière absolue à ceux que nous avons retirés du Psammobia vespertina.

La coquille, vue extérieurement, présente exactement le faciès d'une I'sammobie; bâillement linéare aux deux extrémitẻs, faible carène postéricure, pas de corselet, crochets presque eflacés, ligament élevé, dépassant les crochets; la vue intérieure montre une impression palléale et des impressions museulaires, identiques à celles des Psammobies, une charnière sans dents ou parfois munie d'une dent cardinale rudimentaire, sur chaque valve. N. Agassiz, n'ayant à sa disposition que des moules mal conservés, a classé ces fossiles en partie dans les Mactromya (1) el en partic dans le genre Platymya, el n'a pu donner pour ce genre des caractères neltement défnis; mais en étudiant les deseriptions des especes ainsi que les figures, on parvient très-facilement à elasser les fossiles, surtout quand on a des coquilles vivantes conme moyen de comparaison.

Psamobla axgesti, Terọ. 'et Johrd. PI. Vhil, ligg. 9 et 10.

P. testa tenui, elongrela, ovali, compressa, angusta, utrinque hiante, infro et supra lenitsr arcuatn, antice dimidiata, postice subspatulata, curina plnnulata minuta, plicis angustis, regularibus ornata, umbanibus parvis, vix conspicuis.

Dimensions : longueur $35-54-4$ millim.; hauteur $24-23-20$ millim.; ćpaisseur 14-13-12 millim.; longuear buccale $27-27-21$ millim.; longueur anale $38-35-29$ millim.

Coquille mince, allongée, ovale, étrolte, très-comprimée, bâillante à ses deux extrémités, ornéc de plis concentriques, réguliers et serrés, erochets très-petits, à peine visibles, doncement arquée en dessus et en dessous, un peu rétrécic en avant, élargic en arrière et munie d'une carche peu saillante, atteignant l'angle postéro-inférieur.

Localité : les Clapes; assez commun.

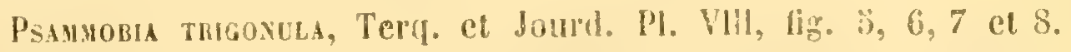

P. Iestr ovali, trigonula, compressa, anlice et postice allenuatr, plicis concentricis, regularibus ornatn; umbonibus mominulis, brevibus, utrinque hiante, infret arculata, supra angulata, anguste carinuta.

Dimensions : longueur 31 - 43 millim.; hauteur $30-24$ millim. ; ćpaisseur 17 - 14 millim. ; longueur buccale 31 - 29 millim. ; longucur anale $33-32$ mill.

Coquille ovale, faiblement trigone, dépriméc sur les côtés, anguleuse el rentlée au côté cardinal, rétrécic en avant et en arriere par la courbure da bord inférieur,

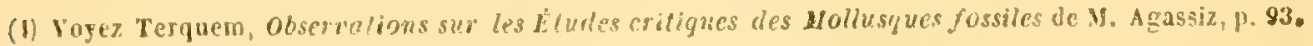


munie diunc carenc saillante pres des crochets, presque eflacéc sur les flancs, ornée de plis concentriques, très-fins, serrés et rézuliers; crochels saillants, courts.

illoule i impressions musculaires et palléale saillantes, lantérieure diviséc en deux parties incigales, la postéricure arrondic dans le bas, anguleuse thus le hant et se conlinuant jusque pres des crochets.

Les moules fig. 7 et 8 de cette espece se rapportent exactement it cents que nous avons retires du l'semmobia vespertina, satuf que les reliefs sont rut rosu plus saillants dans ha coquille vivante.

Loenliti: les Clapes; assez commun.

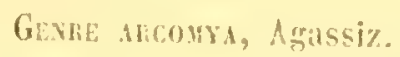

Cormille transverse, équivalve, inéquilatérale, à test ceaillcas, se déăbubint facilement, ornée de plis concentrignes daceroisserient et de lines rotes rayonnantes, gramuleuses, épilermiques ; erockets subantérieurs, airus, fortcment ćchancrés en arrière, repliés en dedans, non perforés à lem extrémitú; pante postéricure bàllante, munie d'un eolselet, linilée par une arète vire ct d'ute carène latcrale; borl coplinal sans dent, muni sous les crochets d'une extavalion verticale, triangulaire, en forme de cuillerun, posterieurement renflé, arrondi à l'intérienr et formant une légère sinuosité pour l'insertion de la nymphe; mymples linéaires, allongées, sćparées du borll cardinal par un sillon; ligrment allongé, gros, s'étendant sous les erochets el s’inserant dans les cuillerons; impressions musculaires, l'antéricure piriforme, un peu approfondie, la postéricure presque

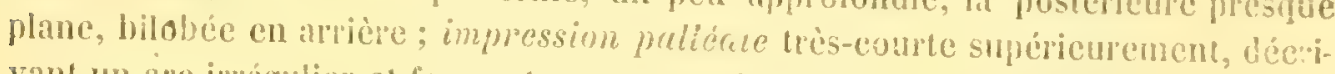
vant un are irrégulier et formant une courte languetle arec le bord infériens.

Nous avons sous les yeux l'dreomya celceiformis, Ag., de Noutio." (Calrados), ef la charniere indique que ce fossile est un llonomya, malgré la presence d"me carene, qui d'ailleurs se troure parfois sur les fossiles de ee dernieg fonre, ce caractère ne pouvant ètre considéré comme générique.

L'évidenent postéricur et caractéristique des crochets, qui vient se confondre avec le corselet, donne lieu sur les motiles à une forte dépression qui est d'autant plus profonde que géncialement le test est plus épais en cel endroit; il en est résullé que, jugés d'après leur forme, beaucoup de ees moules ont été rapuortés aus senre Anatine, bien qu'on n'y remarque pas l'incision caracteristique ies

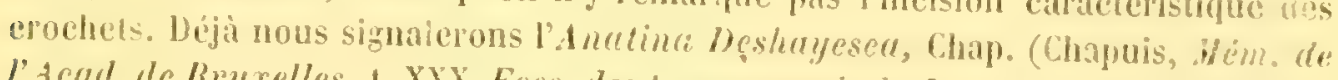

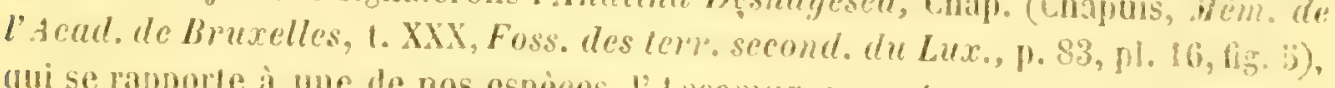
gui se rapporte ì une de nos especes, l'ilicomya cornutu.

Arcoms arguass, Terip. et Jourd.

1\%. 11, fig. a et 10, grandeur naturelle.

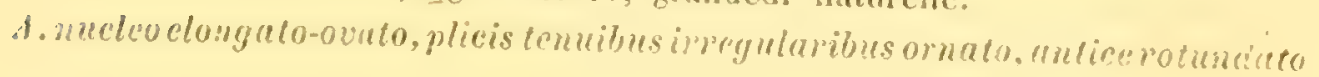


infra regulariter areuato, postice arcuato, hiante, supra utrinque declivo, umbonibus parvis, acutis, inflexis, submedianis.

Dimensions: Iongueur 72 millim.; hauteur 42 millim.; épaisseur 27 millim.; longueur buccale 44 millim. ; longueur anale $\& 8$ millim.

Houle ovale-allongé, muni de plis concentriques très-fins et irréguliers, comprimé sur les cótés, arrondi en avant, régulièrenent arqué inférieurement, arrondi et très-bâillant postérieurement, déclice à droite et à gauche en dessus, corselet très-ítroit, carène latérale, peu sensible, crochets aigus, inlléchis, submédians.

Lnealité : Thiancourt ; asse? rave.

Arcony spatuhati, Tera. el Jourd.

[']. Vl, fig. : et 6 grandeur naturelle.

A. nucleo plicis concentricis regularibus munito, compresso, untice clongato, angusto, infira subrecto, postice producto, spatulato, hiante, supra antice declivo, postice recto, area cardinali minima, curina Irevali angusta, umbonibus acuminatis, in/lexis.

Dimensions : longueur \$8-38 millim.; hanteur 28-97 millim, ; épaisseur 20-18 millim.; longueur buecale $27-23$ millim. ; longueur anale $\$ 1-38$ millim.

Moule orné de gros plis concentriques, réguliers, eomprimé latéralement, allongé et étroit en avant, sensiblement droit inférieurement, allongé, spatulé et bâllant en arrière; botd cartinal très-déclive en avant, droit en arrière; corselet très-étroit, carène latérate n’atteignant pas le tiers de la hauteur du còté postérieur; crochets aigus, inlléchis; impression muscuhire antérieure piriforme, la postéricure ovale; impression palláale d'abord horizontale, puis régulièrement arquéc pour former une longue languelte arec l'impression inféricure.

Cette espèce se rapproche de l'1. cornuta, par la compression générale de la coquille; elle en diffère par sa partic postéricure bencoup plus large ane l'anterieure, disposition qui est inverse dans l'. cormute.

Localité : Longwy (glacis) ; fort rare.

Arconys corveta, Tereq. et Jourd.

P. Vil, lig. 1, 2, 3 et \&, grandeur naturelle.

A. Lesta ovato-elongata, plicis concentricis irregularibus et costulis tenuissimis rudiantibus, nodulosis ophata, lateribus campressu, antice elongata, rotunda, infra leniter arcuata, postice atlenunte, productu, hiante, sup a antice obliqua, postice recta, umbonibus parvis inflexis, area cardinali angusta, obtuse limitala, carina postica vix perspicua.

Dimensions: longueur 63-60 millin.; hateur $38-30$ millim.; épaisseur 2220 millim. ; longueur buecale $32-32$ millim.; longueur anale $42-43$ millim.

Coquille ovale-allongée, à test mince et fragile, ornée de plis concentriques irréguliers et de très-fines côtes noduleuses rayonnantes, comprimce sur les 
cótés, allongée ef arrondie en arant, légèrement arquée dans le bas, très-allongée, rólrécic et bâillante cn arrière; bord cardinal déclive en avant, droit en arrière, inuni d'un corselet étroit, très-aigu près des crochets, limité par une arète obtuse. carène litlérale peu marquée, étroite; crochets très-étroits, aigus, repliés en dedans. ligament allongé, élevé.

Localites: les Clapes, Thiaucourt; assez abondant, mais généralement mat conserve.

ARCOMY INFLATA, Terg. ef Jourd.

II. V, fig. 7 et 8 , grandeur naturelle.

A. lestu ovato-elongata, lamellosa, plicis angustis, irregularibas ormata, lateribus inflata, antice brevi, rolundata, infra oblique arcuata, postice producta, attemuata, hiane, supra recta, area cardinali angusta, obtuse limilata, carina laterali vix perspicua, umbonibus inflatis, depressis.

Dimensions : longueur 73 millim.; hauteur 44 millin. ; épaisseur 36 millim. : longueur buccale 38 millim.; longueur anale 57 millım.

Coquille ovale-allongée, à test lamelleux, munie de plis étroils et irréguliers, renllée sur les côtés, courte et arrondie en avant, obliquement arquée dans le bas, allongée et bâillante en arrière, droite sur le bord cardinal ; corselet étroit, limité par une arête obtuse, carène latérale large, triangulaire, neu sensible, crochets renflés, déprimés et repliés en dedans.

Localité : les Clapes; fort rare.

Arcomy clapensis, Terq. et Jourd.

H. VI, fig. 1, 2, 3 et 4, grandeur naturelle.

A. tesin ovata-elongata, plicis concentricis et costulis nodulosis, radiuntibus, reyularibus exornata, antice brevi, compressa, rotundata, inferne leniter. arcuata, postice producta hiante, superne recta, area cardinali carina acuss limitata, latcribus carina lata, obtusa, ab umbonibus decurrente et mor evanescente instructu, umbonibus angustis.

Dimensions : longueur 80 millim.; hauteur fä millim.; épaisseur 33 millim : longueu buceale 3 m millim. ; longueur anale 68 millim.

Corquille ovale-allongce, à test Jamelleux, munie de larges plis peu saillants e! converte de lines côtes noduleuses, rayonnantes, régulières; courte, comprimée et arrondie en avant, allongée et légérement bìillante en arrière, arquée dans le bas. renflée dans le milieu et suivant la carène; bord eardinal droit, muni d'un corselet plat et limité par une arcte aiguë; carche laterale large, sallante prés des crochets. dirigée vers l'angle inféro-postérieur et peu sensible sur les côtés ; crochets subantérieurs, aigus, étroits, léğèrement déprimés; nymphes étroites, très-allongées. siparces par un sillon; ligament long et arrondi. 
Cetie espece differe de l'd. infaln, par ses crochets plus anterieurs, son corselet mieux limité, par sa carène plus prononcéc et par son prolongement postérieur çui donne à l'ensemble une forme trapézoïdale.

hocalité : les Clapes ; fort rare.

Arconya ingensa, Terq. et Jourd.

Pl. VI, fig. 11 et 12, grandeur naturelle.

A. nucles ovato-slongato, in medio et supra tumido, circiler compresso, qutice pretongo, rotundato, infro areualo, postice abbeviato, attenunto, posirato, area breci, carina vix perspicur.

Dimensions : longueur bै3 millim.; hauteur 31 millin.; épaisscur 21 millim.; fonguetir buceale 37 millim.; longueur anale 27 millim.

Moule ovale-allongé, renflé dans le milieu et près des eroehets, comprimé sur 1e pourtour, còté antérieur plus lonğ que le postérieur, côlé inféricur arqué, côté postérieur très-étroit, en forme de rostre; corselet court et étroit, carène à peine visible, cochets petits, aigus, repliés en dedans.

Eocalite: Wohécourt (Meuse) ; fort rare. (Collection de M. Collie\%).

$$
\begin{aligned}
& \text { Gexres Previony at Hyopse, Azassiz. }
\end{aligned}
$$

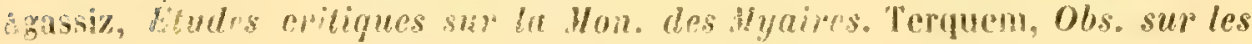

$$
\begin{aligned}
& \text { Étules erit. d'dgassi\%, p. b. }
\end{aligned}
$$

Toutes les coquilles classies da:s ces deux genres, presemant exactement les

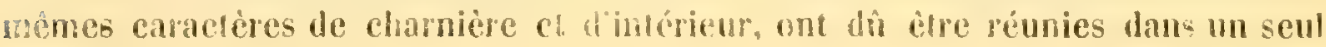
genre.

Coquille équivalve, inéquilatérale ou subęuilatérale, baillante postérieurement ésur le côté ventral, à surface inunic de plis concentriques et ornée de fines côtes granuleuses, rayonnantes, épidermiques; bord cardinal droit ou arqué, poslérieurement la valve droite recouviant la gauche munie d'un biseau; charnicre sans dent, sous les erochets une expansion dentiforme, en gouttière, celle de lit vaive droite reposant sur celle de gauche el suivie par une ouverture triangulaire, formant cuilleron; nymphes fortes et saillanles; impression musculaire antérieure ovale, la postérieure piriforme, impression palléale, occupant la moitić de la fongueur de la conuille, parallèle dains le haut et le bas, régulièrement arquéc dans le milieu et déterminant une languette plus on moins longue, selon te développement plus ou moins grand de la partie postérieure.

Woulc muni d'une depression sous les erochets, celle de la valve gauche deux fois plus grande que celle de la vale droite. 
Plewroma drassi, Al. Brong., sp.

P. 1II, fig. $3,6,7,8$ et 9, trandeur naturelle.

Lutraria jurassi, 11. Brongniart, Ann. eles mines, 1. N, p. biv, pl. 7, lig. 4. Lutraria jurassi, Al. Brongniart, Goldfuss, Petref. Germ., 1. 11, 1. 2:3, pl. 132,

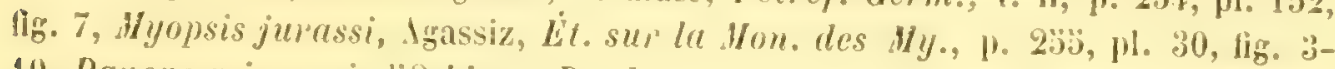
10. Panopara jurelssi, d'Orbigny, Prodrome, 1. 1, 11. 273, n' 209, Myacites jurassi, Quenstedt, Jura, [1. 4.19, jl.61, fig. 13.

La description de celle espèce se trouve bien exposéc par hgassiz el Goldfuss: ce dernier l'indique pour l'oolithe supéricure du Hanore et de Caten.

Localités: les Clapes; trés-abondant; Thiacourl; assez rare.

Pleonomy triendiss, Terq. et Jourd.

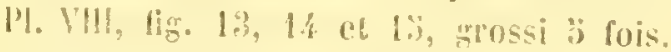

P. testa minima, oblipue ovalu, circiter compressu, in medio gibbosula

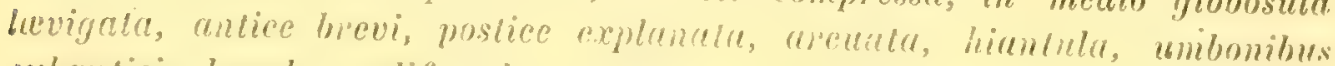
subanticis, lumula cordiformi.

Dimensions : longueur \& mill. ; hateur 2 mill.; chaisseu 1,:3 mill.; longueur buccale 1, 3 mill. ; longueur amale 2,3 mill.

Coquille très-netite, obliquement ovale, comprince sur le pourtour, renflée dans le milicu. lisse, courle en arant, élalèce arrière ct munic d'un bâillement linéaire; crochets subantérienrs. renllés, lunule corliforme.

bien que nous ayons trouré cette espèce associéc aux Lithodomes et aux Saxicaves, nous sommes dans le donte si sa posilion est normale, ou si elle est le résultat d'un accident; d'une pait, nous possédons des Arches perforantes et d'une autre part nous avons, comme nous l'avons dit dans lintroduction, des Limes et des Peignes qui, ayant, ì l'ćlat embryonnaire, été amenés dans des loges vides, ont dù, en giandissant, se déformer, suivant le ereux qui les emprisonnair.

Localité : les Clapes, dans un Synustrea: forl rare.

P'Leuromya chidita, Terel. el Jourd.

1'. 1I!, fig. 10,11 el 12, grandeur naturelle.

1. testa clongatu, amsusta, antieo brovi, areuala, postice producta, hiantula, spantata, carinatu, carina plana, triangulari, infra longe arcuata, sinuata, supra utrinque leniter obliquatu, umbonibus inflatis, infexis, in lertia antica parte silis, area cardinali angusta.

Dimensions : longueur 49-31 millim.; hantenr 20-13 millim.; épaisseur 1:i-9 millim.; longueur buceale $22-13$ millim.; longueur anale $32-22$ millim.

Coquille allongée, étroite, deux fois et demi plus longue que haute, courte ef arquéc en avant, allongée, spatulée, earénéc, i carène plane, triangulaire el

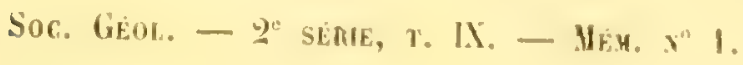


munic d'un bảillement linéaire en arrière, faiblement arquée et sinueuse inférieurement; bord cardinal plus déchive en avant qu'en arrière, crochets renflés, repliẻs en dedans, corselet étroit.

Localité : les Clapes; fort rare (Coll. de H. de Lambertye).

Pleuromis maginata, Ag. Sp.

Myopsis marginata, Agassiz, Hon. des Myaires, p. 237, pl. 30, fig. 1-2.

tgassiz fail observer que " celle espèce a les mèmes dimensions et la même physionomie que le 1 . jurassi; mais elle est plus allongéc, le bord inférieur est plus droit; les erochets sont aussi d'ordinaire plus prépondérants et les flanes plus aplatis."

Un de nos échantillons n'a pas de sillon ventral, un autre est très-court et senflé.

Loealités : les Clapes; assez commun; Gravelotte (marnes); fort rare.

\section{Pleuromya Onalise, Chap.}

P. VII, fig. 19, 20 et 21 , grandeur naturelle.

Chapuis, Mém. de l'Ac. de Brux., t. XXXII, Foss. des lerr. second. du Lux., 11. 67. 11. 13, fig. 4.

Cette espèce est munie du sinus ventral du $P$. decurtata, Ag., et présente l'élévation du $P$. jurassi, mais elle est plus comprimée et proportionnellemen plus courte.

Localité : Ies Clapes; fort rare (Coll. de H. de Lambertye).

Peevaomisa flovgita, Mu. Sp.

Lutraria elongata, Hunster in Goldfuss, Petref. Germ.; t. 1!, p. 258, pl. 153, fig. 4, a, b. Pleuromya elongata, Agassiz, Mon. lles Jyaires, p. 244, pl. 27. fig. 33-8. Panopea subelongata, l'Orbigny, Prodrome, 1. I, p. 272, n² 208.

Cette espèce possède un test foliacé, orné de plis concentriques, très-irrréguliers, qui se reproduisent sur les moules; elle fournit plusieurs variétés à formes plus on moins rentlées. Les erochets sont placés an tiers antérieur et le bord cardinat présente une double déclivité plus forte en avant qu'en arrière.

Localités: les Clapes, Gravelotte, (marnes jaunes et bleues); Thiaucourt, Fontoy, Longwy (glacis); partout très-abondant.

\section{P'echoms iocita, Phil. Sp.}

- Mya requala, Mhillips, Cieol. York., pl. 11, tig. 12, Myaciles requatus, Phill. Sp. Morris et bycelt, Moll. de la grande oolithe de l'dngl., p. 139, pl. 12, lig. 5. Panoper aequata, dophigny, Prodiome, t. 1, 1. 2273, n" 217.

Inealité : Cirarchote (marnes); fort rare. 
P'cectrohys ovals, Hu. Sp.

Lutraria ovalis, Hunster in Goldfuss, Petref. Germ., 1. 1I, P. 257, pl. 15:3. fig. 1, a, b. Pleuromya nenlis, Agassiz, Mon. des Myoires, 1. 234, Panopaa subovalis, dorbigny, Prodrome, 1. 1, 1. 273, $1^{0} 220$.

Localités : Ies Clapes, Longwy (galacis); fort rare.

Mreqroms textistha, He. Sp.

Latraria temialra, Wunster in Goldfuss, Petref. Germ., 1. 11, 1). 237, ph. 133,

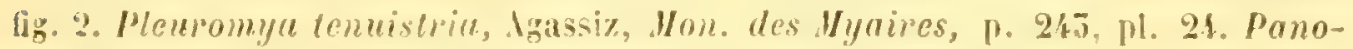
pea tenuistria, d'0rbigny, Prodrome, 1. 1, p. 273, 11 212.

Goldfuss fait observer que les caractires de l'espice sont tirés des lines stries nodulenses rayoumantes; Agassiz les mend daus les rides concentriques, plus fines ot plus serrées que daus les autres especes.

localites: Gorze (cimetièn); assez rare; Thiaucourt; très-commun.

\section{Phethomy decumati, Phill. Sp.}

Amphidesma decuratum, Mhill. Feal. York., pl. 7, fig. 11. Lutraria decurtata,

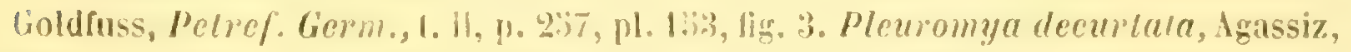

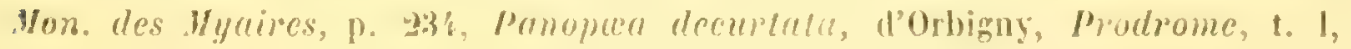
p. $273,113296$.

(ioldfuss figure une valte droite et fait mention 'p. wis) d"une expansion denliforme horizontale (1); mais ne possédant pas la ralse opposéc il n'a pu en apprécier la valeur, ni indifuer te caractère exeeptionnel du genre.

Localité : Gravelotte (matues); très-commun.

l'bechousa giobati, Terqg. et dourd.

14. K1, tig. 16, 17 et 18, ermentem naturelte.

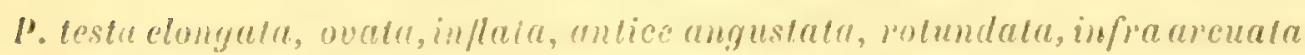
non sinuata, supre anlice obliquure, postice recta, umbonibus subanticis, inflatis, incurvis.

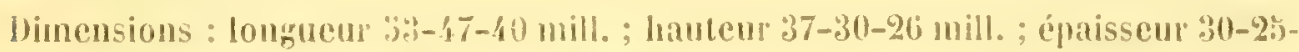
20 mill.; longueur buceale $: 30-27-29$ - mill.; longueur anale $48-38-33$ millim.

Coquille allongée, ovale, renflée, un nen rétrécic et arrondie en avant, arquéc et sans sinus inférieurement, arrondie et munic d'un bàillement linéaire en arrice; bord carlinal declive en avant, droit en arrière: crochets, renfles,

(1) Bei einer Schale, ilercn Schlossranl entblust werde, macht sich ein Regelformiger, senkrechler Eidnruch, und eine Leiste zur Anlagerung eines ieussern Bandes bemerklich. Es verrueth also dieses Schlossrudiment

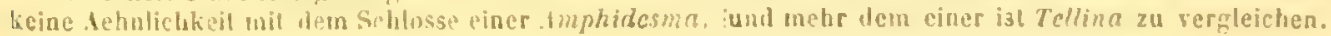


repliés en dedans, situés au quart antérieur; expansion dentiforme de la valve żauche allongée et oblique en arrière; celle de la valve droite très-courte.

Cette espèce, par sa forme ovoïde et renflée, sans sinus ventral, se distingue de poutes les autres.

Localité : les Clapes; très-commun.

Pleunomy gricilis, Terrf. et Jourd.

M. VII, fig. 13, 14 et 13 , grandeur naturelle.

P. testu elongata, angusta, gracili, antice rotundata, infra antice sinuata, in medio areucta, deinde attemuta; postice producta, subcarinata, hiantula, supra subrecta; umbonibus parvulis, inflexis, in tertia antica parte sitis.

Dimensions : longueur 30-41 millim. ; hauteur 23-21 millim. ; épaisseur 19-14 millim.; longueur buceale 23-22 millim.; longueur anale 33 -30 millim.

Coquille allongée, étroite, grèle, arrondie en avant, sinueuse puis arquée ę relevée inférieurement, allongéc, subcarénéc et munic d'un bâillement linéaire en arrière, presque droite en dessus; crochets pelits, déprimés, repliés en dedans, placés au tiers antérieurs.

Les moules se distingucut par leur partie postéricure spalulée; les impressions musculaires forment une légère saillie, toutes deux sont aiguës à leur partie supérieure, l'impression palléale est marquée par un léger sillon.

Cette espece, par la forme générale de la coquille, se rapproche du $P$. elonguta et s'en éloigne par une moindre élévation, par son bord cardinal droit, au lieu d'être déclive des deux côtés, et par sa parlie postéricure disposée en rostre.

Localités: Ies Clapes, Gorze (cimctière); assez commun; Gravelotte; fort rare.

$$
\begin{aligned}
& \text { Gevre Gressiya et Cenomya, Ag. } \\
& \text { Igassiz, Mon. des Myaires, p. 202, et pl. } 25 .
\end{aligned}
$$

Coquille très-inéquilatérale, inéquivalve, la valve droite plus élevée que la gauche et la recouvrant sur toute la partie supéro-postérieure, suborbiculaire ou transverse; crochets antérieurs inégaux; charnière sans dent, asymétrique; sur la valve droite et sous le erochet une callosité termince par un cuilleron; contre le bord cardinal et en dedans une longue nerrure qui sert de nymphe; sur la valve gauche et sous le erochet, un cuilleron plat; sur le bord cardinal une gouttière dont le bord élevé sert de nymphe ; ligament caché par l'expansion de la valve droite taillée en biseau en dedans; bord de la valve gauche en biseau en dessus; impressions musculaires marginales, sémilunaires ou subaiguës dans le haut, sinus palléal formé par un anšle à sommet arrondi.

Houle marqué d'un sillon qui confourne te erochet droit et résultant de la saille de la nymplic. 
Ces caractères, étant en tous points communs aux deux genres, permeltent de les réunir en un seul et de ne conserver que le genre Gresslyre (1). Nous ne connaissons que deux caracteres, quoique super/iciels, qui puissent serrir à distinguer les Céromves des Gresslyes; dans ceux-ci, la coquille est ovalaire et les erochets ne dé sent pas le côté antérieur; dans ceux-là, la coquille est complétement transverse el les crochets dépassent le coté antérieur.

$$
\begin{aligned}
& \text { Groseli ericis, Ig. } \\
& \text { Srassiz. Mon. Ules Myares, p. 214, pl. 14. fir. 1-9. }
\end{aligned}
$$

Localités: Gravelotte, Longry ; assez rare.

$$
\text { Gresstid zovith, in. }
$$

Agassiz. Mon. des Hyaires, p. 214, pl. $12 \mathrm{~b}, \mathrm{lig}, 1-3$. Localités : les Clapes, Grayclotte, Longwy; fort rare.

$$
\text { Ginessly. troxcata, Iro. }
$$

Irassiz, Mou. des Myctioes, p. 21:3, 11. 12 b., fig. k-6.

Loealités: les Clapes, Longwy, Grarelotte; partout très-abondant.

GRESSLI LUTLLATA, Ag.

Agassiz. Mon. des Myarires, p. 208, pl. 13, fig. \& à 10, pl. 13 a, fig. 1-1

et pl. 1\% 1), fig. 7-9.

Localités : les Clapes, très-abondant; (iravelotte, fort rare.

Gresslya latior, dg.

Igassiz. Mon. Iles Hyaires, 1. 210, pl. 1:3 b, lig. 10-12.

Localitćs : les Clapes, Gravelotle; fort rare.

Giressigi hostrata, Ig.

Igassiz. Von. des Hyaires, p. 211, 11. 12 b, lig. 7-9.

Xous sommes dans le doute si les moules que nous rapportons à cettr espèce se trourent exactement classés; ils semblent établir des passages au G. truneala.

Loealité : Longwy (les glacis); asse\% bare.

Biesstya coscritnica, Ino

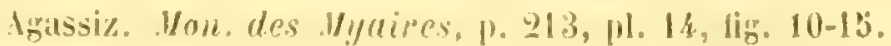

Localites: Gravelotte; assez commun; Longwy; assez rare.

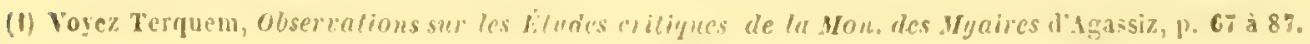




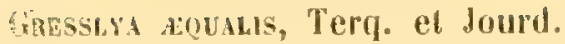

Pl. UIII, fig. 1 et 2 ; grandeur 1/1.

G. nucleo orbientari, subequilaterali, antice quam pastice majore, circiter compresso, in medio tumidn, umbonibus submodianis, inflatis, inflexis, concortis.

Dimensions : longueur 31 millim.; largen. 28 nillim.; ipaisseur 18 millim.; longueur buccale 22 millim.; longuear anale 23 millim.

Moule orbicuhire, mesque aussi haut que large, subéquilatéral, le côté antérieur un pes plts large que le postérieur, orné de côtes concentriques régulières; compriné sur tout le pourtour, renllé dans le milieu; crochets submédians, renflés, repliés en dedans et contournés, te crochet droit marqué du silion caracteristique.

Celle espece, foiqu'ì létat de moule, nows a part très-intéressante, en ee qu'elle semble établir e bassage de la forme allongée ct comprimée des Gresslyes à la forme branserse plus arrondie et plus renllée des Céromyes.

Localite : Thiancusert; fort rare.

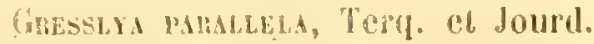

13. VIII, fig. ; et 4, gramdeur $1 / 1$.

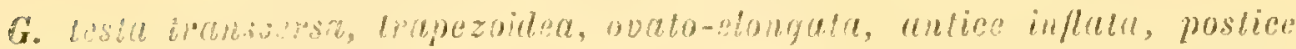

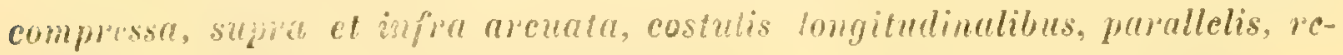
gularibus, conferlis ornate, umbonibus anlicis, tumidis, prominentibns, involutis, dextro quen sinistro ampliore.

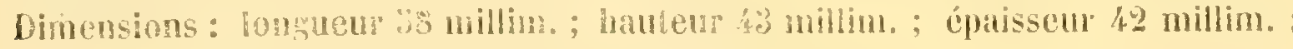
Iongueur buecale 21 nillim. ; longnem! anale 88 millim.

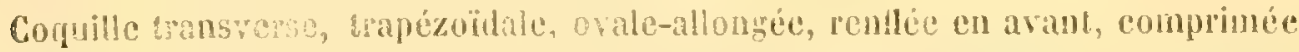
en arière, arguée bons le haut ef te bas, ornée de côtes longitudinales, régulières, parallèles et serrées; crochets anterieurs, rentés, saillans, curoulés, le crochel droil plus gros que celui de gauche.

Cette espèce, gử appartient à la fivision des Céromyes à forme transversate, est la première do genre qui apparait à ce niveau, toutes les autres appartiennent à l'oolithe moyenne el à la supérieure.

Localité : Wohécant (alleuse); fort rare (Coll. de I. Colliez).

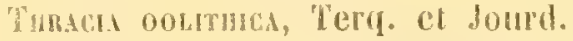

4. V111, fig. 11 et 12, grandeur 111.

T. test subtrigona, depresst, incogulaterali, concentrice multiplicatu, antice rolundata, infra areusta, postice compressa, expansa, supra angulatu, umbonibus submeaianis, carince antica vix conspicna.

Dimensions : longueur 41-34 millim.; largeur 39-26 millim.; épaisseur 16-1:3 millim. ; longueur buccale 31 -28 millim; longucur alale $28-24$ millim. 
Coquille subtrigone, comprimée, inéquivalve, inéquilaterale, ornée de plis concentriques, irréguliers, arrondic en avant, dépriméc en arrièce, irrégulièrement anguleuse sur to bord cardinal; crochets submedians, carene anterieure peu saillante.

Vous possédous deux cehanthlons, l'un avee son test of so charnière. l'autre à l'état de moule, et auenn ne se rapporte aux especes figures par lgassiz et qu'il a réunies dans son genre Cercomya (1).

Localités: Lonrwy, (olacis), Amanvillers; fort rare.

Sixicili nostmita, Tery. el Joird.

P. VIll, fig. 23, 24 et 25, grossi 3 fois.

s. besta minima, ovata, plicis irregularibus ornua, antice brevi, molindala, infra liarcuata, in inclio sinualu; postice moducta, hiantuln, compressa, roslrata, supra recta, lunula magna, cordiformi, carma grosica spinifera, ab nmbonibus derumente, umbonibus parvulis, inflatis, depressis, inflexis.

Dimensions : longueur 7 milin.; Lauteur 5 millim, ; cpaisseur 2,3 millim.; longneur buecale $2, ;$ millim. ; longuen anale 6 millin.

Coquille très-petite, ovale-allongée, ornéc de plis coneentriques irréguliers, côté antéricur arrondi et muni d'une lunule profonde, cordiforme, côté inférieur formé de deux ares séparés par un profond sinus, côté postéricur allongé, parfois relevé, comprimé, muni d'un bàillement linéaire eq d'une large carène transversalcment costellée el limitće par wne arète aiguë, ornée d'épines; bord cardinal droit ; crochets petits, renthés, déprimés et intléchis

Un échantillon a sa partie postérieure en forme de rostre relevé, dépassant ie bord eardinal. Celte espèce, avec les suivantes, se trouve dans des polypiers avec les fiastrochones, les Lithodomes et les Irehes; les logros sont renbilies d'une marne jaune, assez tendre, qui enveloppe les fossiles.

Loealité : les Clapes, dans les Istseren Conybeari et seriobis; assez lare.

Saxicala biseolati, Terf. el fourd.

P. VIII, fig. 26, 27, 28 et 29, grossi 2 tois.

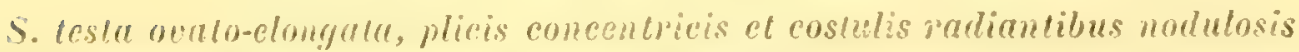
ornata, antice brevi, arcualu, late lunulata, infra recta, in medio subsinunta impressu, postice areuatu, compressa, hiantula, acule carinata, supra abcuata, nimbonibus parvis, inflexis, cardine indentato.

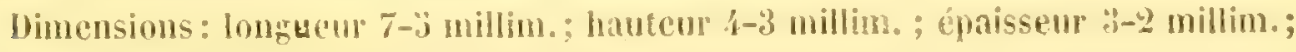
Iongueur buceale $3-2,3$ millin.; Iongueur anale $6-4$ millim.

Coquille ovale-allongée, ornéc de plis concentriques, irréguliers et de lines côtes rayonnantes gramuleuses, còté antérieur court, rentí, muni dune large

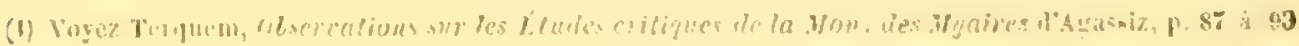


lunule peu profonde; cóté inférieur droit, légèrement sinueux dans le milieu, Ilanes déprimés, côté postérieur comprimé, ar̃qué, faiblement båillant, inuni d'une carène limitée nar une arête aiguë, còté supérieur arqué; crochets subantérieurs, petits, infléchis; charnière sans dent.

Cette espèce se distingue par son côté antérieur très-court et par la dépression des flancs, qui rend le còté antérieur' el la carène d'autant plus saillants.

La coquille est ornée de fines côtes granuleuses, comme les Areomyes, les Pleuromyes et les Gresslyes, ornements qu'on retrouve d'ailleurs sur les Panopées fossiles de Dax.

11 resterait à rechercher si ces ornements sont simplement propres à l'espèce ou s'ils appartiennent au genre et s'ils se trouvent de même sur les coquilles vivantes; toutefois on les voil très-distinctement sur 4 de nos échantillons, mais nous les avons vainement cherchés sur les autres especes fossiles dont les coquilles sont non moins bien conservées, ainsi que sur quelques espèces vivantes.

Localités : les Chapes, arec les précédentes; assez commun.

Saxicaya globosa, Terg. el Jourd.

1\%. VIII, fig. 18 et 19 , grossi 2 fois 112 .

S. testa nuala, globosa, pliris imegularibus, concentricis ornata, infra antice et postice areuta, lateribus inflata, supra antice magis quam postice obliquata; hmule vix perspicun, rerina postice obtusa, umbonibus parvis, obiusis cardine indentatn.

bimensions: longneur 8,3 millin.; largeur li millim.; épaisseur 4 millim.; longnen buccale $4, ; 3$ millin. ; longucur anale $6, \ddot{6}$ millim.

Coquille ovale, globuleuse, oruée de plis concentriques irréguliers, arrondie en arrière et en avant, régulièrement arquéc dans toute la partie inférieure, renflé dans le rnilieu; munie d'une lunule à peine visible et d'une earène limitée par une arête obtuse; crechets netits, obtus; charniere sans lent.

localité : les Clapes, aree les préécilentes; lort rare.

Saxicava ovifohis, Tery. el Jourd.

PI. VIll, fig. 16 et 17 , grossi \& fois.

S. testa oviformi, lavigata, nitida, antice abbreviata, inflata, lunulu minima, infra et supra leniter arcuata, postice rolundatu, angustissime carinata, umbonibus tumidis, inflexis, anticis.

Dimensions : longueur bु, 5 millim.; hauteur ä millim.; épaisseur 3 millim. ; longueur buccale $2,: 3$ millim.; longueur anale 3,3 millim.

Coquille oviforme, lisse et brillante, droite et très-courte en arant, arquée dans le haut et le bas, munie d'une lunule très-étroite et d'une carène superficielle; crochets reụflés, infléchis, antẻrieurs. 
Cetté espece diflère du.S. globosa par ses crochets antérieurs, par sa lunule étroite et par sa carène à peine indiguée.

Localité : les Clapes, avee les précédents; fort rare.

Saxicari ovils, Terg. et Jourd.

PI. VIII, fig. 20, 21 et 29, grossi 2 fois et $1 / 2$.

S. testa ovali, leviguta, antice arcuuta, compressa, parve lumulata, postice producta, leniter carinata, infra recta, in medio impressn, supra angulata, utrinque obliquata, umbonibus tumidis, inflexis, antemedianis.

Dimensions: longueur 8 millim, hauteur ä millim.; épaisseur à millim。; longueur buccale \& millim.; longucur anale 6 milim.

Coquille ovale, lisse, comprimée dans son ensemble, arrondie en avant, allongée en arrière, droite dans le bas et légèrement sinueuse dans te milieu, renflée dans le haut, à bord cardinal oblique des deux côtés, lunule petite, earène à peine indiquée; crochets rentlés, infléchis, placés at tiers antérieur.

Cétte espèce, par sa forme compriméc et sa faible carène, s'éloigne de toutes celles que nous venons de décrire; elle se rapproche du S. arenicola, Terq. (Paléont. de Ilettange, p. 69, pl. 18, fig. 7.) par la forme générale de la coquille, ét s'en éloigne par une disposition trigone et par un moindre prolongement postérieur.

Localité : les Clapes, avec les précédents; fort rare.

Isodoxta Buviguer, Terq. el Jourd.

P. VIII, fig. 30, 31 et 32 , grandeur naturelle.

\%. testa subaruilaterati, svata, inflate, rotundata, costulis concentricis, tenerrimis, strictis ornate, antice rolundala, infra leniter arcuata, postice subangulata, late carinata, carina oblique transversim plicata, costula acuta lin itata, umbonibus parvis, obtusis, inflexis, medianis.

Dimensions : longueur 17 mill. ; hauteur 10 millim. ; épaisseur 12 millim.

Coquille subéquilatérale, ovale, renllée, arrondic, ornéc de très-fines cótes concentriques, régulières, très-serrées; arrondic en arant, légèrement arquée dans le bas, angulcuse en arière, arquée au bord carlinal; munic d'une large carine ornée de plis transverses et obliques et limitée par une arète aiguë; crochets petits, obtus, repliés en dedans, médians; impressions museulaires profondes, impression palléale formce d'un are régulier et n'oceupant que le tiers de la conuille.

Cette espèce diffère de 1\%. Deshayesea, Bus. (Buvignier, Allas de la Géol. de la Mcuse, p. 11, pl. 10, firg. 30 à 3,3$)$ par ses ornements et par sa carène aiguë; de 1'I. Engelhardti, Terq. (Terquem, Pateont. dugrès de Iletlange, p. 73, pl. 18, lig. 14) par les mènes caractères el par sa forme rentlée el arondie.

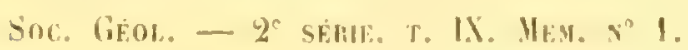


Morris et Lycelt, adoptant te genre Sowerbya, créé par d'Orbigny pour une coquille oxfordienne à laquelle il attribue un cuilleron au lieu de nymphes, ont publié un I. triangularis (Mor. et Lyc., Paléont. de la Gr.ool. de l'Angl. Supplément, p. 66, pl. 33̆, fig. 3.) qui se rapproche de l'I. Buvignieri par ses ornements et s'en éloigne par des crochets plus élevés, qui donnent une forme triangulaire d̀ la coquille; $1 \%$. Woodwardi Ilor. el Lye., (ibidem, p. 67, pl. 40, fig. 27 ) est aplati et se rapproche ainsi de l'I. Engelhardli de lleltange.

Localité : les Clapes; assez commun.

Opis similis, Sow. Spp.

Cardita similis, Sowerby, Min. Conch. p. 280, 11. 232, fig. 3, Opis similis, Morris et Lyectt, Moll. de la Gr.ool. de l'Angl. 1. 81, pl. 6, tig. 4.

Localité : les Clapes; assez commun.

Opis pUlchella, d'Orb.

Cardila lunulala, Sowerby, Min. Conch., p. 279, pl. 232, fig. 3, non fig. 1, 2, 4, 3. Opis pulchella l'Orbigny, Prodrome, t. I, p. 307, n 193. Opis pulchella, Morris et Lycett, Suppl. L. C., p. 61.

b'Orbigny a indiqué cette espèce dans le Prodrome sous cette courte description: espèce voisine de l'O. lumulata; mais bien plus courte et moins oblique, presque carrée, ornée de côtes concentriques.

Morris et lycett ont, dans leur supplément, rectifié leur première détermination ( L. C., P. 80, pl. 6, fig. 3.) at admis l'espèce de d'Orbigny.

D'Orbigny phace l'O. lumulata dans le Bajocien et le Pulchella dans le Bathonien. Localité : les Clapes; assez rare.

Opis rusrica? d'Orb., pl. IX, fig. 1, 2 et 3̈.

D'0rbigny, Prodrome, t. 1, p. 308, n" 196, Bathonien. "Espèce plus ląge que haute, renflée, plane en dessus et en dessous, et comme évidée sans excavation lunulatie."

o. testa crassa, inflata, trigona, transversa, costis regularibus, elatis, Iransversalibus, interstitio amplioribus ornate, antice fornicata, angulo rotundato, infira recta, postice truncata, angulo acuto, carina lata, plana, umbonibus magnis, anticis, elatis, involutis, lunula nulla, margine interno erasse dentato.

Dimensions : largeur 16 millim.; hauleur 14 millim. ; épaisseur 2 f millim.

Cocquille à test épais, rentlée, plus large que haute, trigone, transverse, ornée de côtes transversales, élevées, régulières, Hus larges que lés intervalles; verticaic et excavée en avant, à angle arrondi, droitedans le bas, tronquée en arrière, à angle aigu; carène large et plane ; crochets épais, élevés, enroulés en dedans, Junule non marequée; bord intérieur mun d'une séric de grosses dents. 
Cette espèce se distingue de l'o. similis par sa partie inféricure horizontale.

Nous sommes dans le doute si notre espèce se rapporte exactement à celles que d'Orbigny indique avec ce earactere, dont nous ne saisissons pas la valeur: - plane en dessus et en dessous o; nous asons eru en conséquence devoir en donner une description complète.

Locatité : les Clapes; assez rare.

Astaite Derressa, MU.

Munster in Goldfuss. Pelref. Germ. 1. 11, p. 192, pl. 134, tig. 14, a, b. c.

Cette espèce parait asse $\%$ variable dans sa forme; un échantillon se rapporte exactement à la figure de Goldfuss; un autre est déprimé à sa partie antérieure; un troisiène est proportioncllement beaucoup moins élevé el possêde plus de longueur.

Localité : les Clapes; asse\% rare.

$$
\begin{aligned}
& \text { Astarte elegals, sow. } \\
& \text { sowerby, Jin. Conch., p. } 83, \text {, pl. 137, fig. } 3 .
\end{aligned}
$$

Celle espèce se présente également dans l'assise sous-jacente au fuller's, dans le calcaire ferrugineux et dans le calcaire a polypicers; mais c'est aux clapes qu'on la trouve avee sas ornements les mieux caraclérisés.

Localité : les Clapes; assez commun.

Astarte misisa, Phil.

Phillips, Gícol. du York, pl. 9, fig. 23.

Localité : (irarelotte; très-abondant dans le calcaire de Jaumont, fort rare dans les marnes bleves.

Astarte detrita, Golde.

Golduss, Petref. Germ., 1. 11, P. 191, p1. 134, lig. 13.

Celte espèce, plus rentléc et plus épaisse que l'A. elegens, s'en distingue par ses côtes simples, trois fois plus étroites que les intervalles; ceux-ci sont lisses.

Localité : les Clapes; asse\% commun.

Istakte struto-costata, Vu.

Munster in Goldfuss. Petref. Germ., t. II, p. 192, pi. 134, fig. 18.

Localite : (iravelotte, (marnes); fort rare.

Istirte momath? Walt.

Walton, U. S. S. Horris et Lycell, Suppl. des Hol. de la Gr. ool. de l'Angl., p. $77, \mathrm{pl} .40$, fir. 34 .

localité : les Clapes; fort rare. 


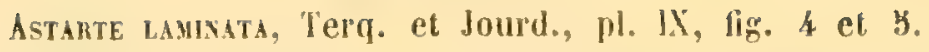

A. testa transversa, ovata, maxime compressa, velut laminata, subplana, circiter depressa, costulis obtusis, concentricis, regularibus, arcuatis ornata, interstitiis conformibus striatis, umbonibus parvis, planis, subacutis, antemedianis, lunula profunda, angusta, area ligamentilonga, excavata, margine interno indentato.

Dimensions : largeur 50 millim.; hauteur 34 millim.; épaisseur 9 millim.; longueur buccale 23 . ; longueur anale 40 millim.

Coquille transverse, ovale, très-comprimée, plane, déprimée sur le pourtour, ornée de côtes obtuses, concentriques, régulières, les intervalles égaux et couverts de stries; crochets antemédians, petits, aigus, lunule profonde et étroile, fossette ligamentaire allongée et profonde, bord interne lisse.

Localité: les Clapes; fort rare (Col. de H. Colliez;.

Astarte carinata, Terq. et Jourd.; pl. IX, tig. 6, 7 et 8.

A. testr subtrigona, tenerrime, concentrice costulata, antice et postice rotundata, antico magis quam postico angulo majore, infra recta, leniter carinata, supra utrinque decliva, in medio inflata, circiter compressa, lunula parva, cordiformi, area cardinuli angusta, lanceolata, umbonibus medianis, parvis, rectis, margine iniemo circiter dentato.

Dimensions: longueur 18 millim.; hauteur 13 millim.; épaisseur 9 millim.; longueur buccale $\{1$ millim.; longueur anale 16 millim.

Coquille subtrigone, ornée de fines côtes régulières, concentriques, plus serrées près des crochets ugu'urférieurement, arrondic en arrière et en avant, angle postéricur plus petit que l'antérieur, droite dans le bas; munie d'une faible carène, renflée dans le milieu, comprimée sur le pourtour, lunule cordiforme, petite, corselet élroit, lancéolé, crochets médians, rentlés, droits, bord interne muni de dents sur le pourtour.

Celte espèce diffère des autres par ses ornements très-fins et réguliers, par son renflement dans le milieu et par sa carène.

Localité : les Clapes; assez commun.

Astarte inequicostata, Terq. et Jourd. pl. IX, fig 9 et 10.

A. testa subtrigonula, inflata, lumuli angusta, lanceolata munita, costulis slrictis, regularibus, mox crescentibus ormuta, circiter compressa, supra tumidu, antice brevi, postice producta, angusit, infra leniter arcuata, antico quain postico angula ampliore, umbonibus subunticis, parvis, obusis, margine interno dinticulato.

bincnsions: longueur 21 millim, ; hautcur 16 millim. ; épaisseur y millim.; longueur buceale 13 millim. ; longueur anale 17 millim. 
Conulle subfrigone, rentice pres des crochets, compriméc sur fe pourlour, munie d'une lunule étroite, lancéolec, ornée tres-fines cotcs, scréces, régulieres, devenant plus grosses et plus espacées avecl'aceroissement de ha coquille; courte en arant, doucement arquéc dans le bas, allongée, trés-dédive el alténuće en arrièce, angle antérieur plus grand que le posterieur, crochels subantéreurs, petils, obtus, bord interne dentieulé.

Localile : les Clapes; assez mare.

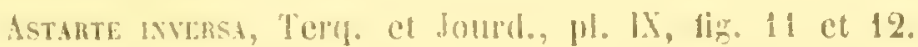

A. Lesta inegulariter subirigona, compressu, planulata, lunula cordiformi instructa, costis concentricis, areualis aut angulatis, obusis, conformibus ornata, antice infra arcuata, supra sinuula, postice obliqualu, potunduta, infrareclu, in medio subimpressa, umbonibus subanticis, parvis, subacuminatis, margine interno denticulalo.

Dimensions: longueur 23 millim.; hauteur 19 millim.; épaisseur 9 millim.; longueur buceale 14 millim.; longueur anale 22 millim.

Coquille irrégulièrement trigone, compriméc, aplatie sur loute sá surface, munie d'une lunule cordiforme et d'un eorselet étroit; ornée de côtes concentriques, obtuses, arquées dans le haut, anguleuses dans le bas; droite inférieurement et légèrement sinueuse dans le nulicu, à angles arrondis, le postérieur plus large que l'antérieur, exeavée cn avant, oblique en arrière; crochets subantérieurs petits, subaigus ; bord intérieur limement denticulé.

Cette espece a la forne compriméc de l'A. exilis el en dillère par ses crochets qui ne sont pas médians; elle se rapproche ansi de l' inceruistriata el s'en éloigne par son aplatissement, par ses ornements et par son angle postérieur plus fort que l'antéricur.

Localité : les Clapes; fort rare.

Astarte pisolina, Telu. el Jourd., pl. IX, fig. 13 et 14.

A. testa suborbiculari, gibbosu, circiter altenuata, costulis cancentricis, conformibus ornalu, umbonibus medianis, lunula parva, cortiformi.

Dimensions : longueur et hauteur 9 millin.

Coquille suborbiculaire, rentléc, alténuée sur son pourtour, ornće de còtes con. centriques, égales aux intervalles; crochets médians, lunule petite, peu profonde, cordiforme.

Localice : Thiateourt; assez rare, surtout arec le test.

Istakte Exh.IS, Tery. el Jourd., pl. IX, fig. $1 \%$, 16 el 17.

A. lesta subtrigona, compressa, planulata, angusle lumulata, costis concentricis, obtusis, interstitio majoribus ornata, margine cardinali utrinque declivo, infra recla, angulis oblusis, umbonibus meciianis, purvis, subaculis, margine incerno subtilissime dentalo. 
Dimensions: longueur 14,j-12 millim; hauteu 1\$,3-8 millim.; épaisseur 8-ì millim.

Coquille subtrigone, tres-comprimce, aplatie sur toute sa surface, ornéc de côtes concentriques, obtuses, plus larges que les intervalles, bord cardinal déclive des deux côtés, bord inférienr droit, à angles arondis; crochets médians, petits, subaigus, lunule courte, élroite, lancéoléc, bord intérienr finement denticulé sur tout son pourtori:.

Localité : les Clapes; Irès-commun.

Istarte grata, Terq. el Jourd., H. IX, fig. 18, 19 el 20.

A. tesla orbiculari, inflala, subcarinatr, lunula cordiformi instructr, costis spissis, concentricis, trapeziformibus, duabus minoribus in interstitis ornata. umbonibus inflatis, medianis, margine interno dentato.

Dimensions : longueur 6,5 millim.; hauten 6 millim.; épaisseur 5,5 millim.

Coquille orbiculaire, rentlée, subcarénée, munic d'unc lunule cordiforme, profonde, ornée de còtes clevées, trapéziformes, concentriques, obtuses, et de deux plus petiles dans les intervalles; còté postéricur tronqué dans le bas suisant la largeur de la carêne; crochets renllés, méllans; bord intérieur muni de grosses dents.

Lucalité : les Clapes; tres-commun.

Astarti raucicosta, Terig. el Jourd., pl. IX, fig. 21.

A. testa premula, ovato-quadiungulare, liansversa, compressa, bे-6 costis elulis, sparsis, angulosis ormatr, umbonibus subanticis, lumula pavvulu, vix perspicua, margine interno postice erasse dentonlo.

Dimensions: longueur 10 millim.; hateur 6 millim.; épaisseur 4 millim.

Coquille petite, ovale-quadrangulaife, transverse, comprimée, ornée de ỹ ou 6 côtes élevées, espacées, anguleuses, à intervalles lisses, munie d'un large corselet el dune lunule a peine visible; crochets subantérieurs, bord interne garni postélicurement de puelques grosses dents.

Cette espice se rapproche de I'A. interlineala, Lye. sp. (Horris et Lycelt, Mol. de la gr. ool. de l'Angl., p. 87, pl. 9, fir. 14 et lij) par sa forme générale; elle s'en distingue par sa partie postérieure qui n'est pás obliquement fronquée et par les intervalles des côtes qui sont lisses

Localisé: les Clapes; forl rare. (Col. de II. de Lambertye et de M. Colliez.j)

Astarte paperats, Terq. et Jourd., pl. IX, lig. 22 el 23.

A. Lcsla suborbiculari, circiler compressa, in medio inflata, costis arcuatis, conformibus ornata, supra utrinque decliva, antice, infro el postice regulariler arcuata, umbonibus parvis, acutis, medianis, lumula elongata, cordiformi, margine interno dentato. 


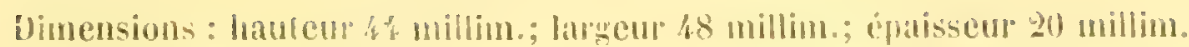

Coquille suborbiculaide, compriméc sur le pourtour, renflée dans le milieu, ormé de coltes concentriques, regulières, érales aux infervalles; déclive des deux

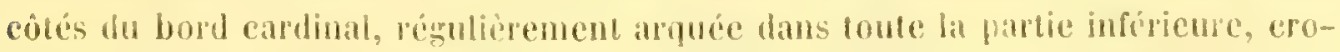
chets médians, pelits, atgus, lumule allongéc, condiforme, corselet étroit et profond, bord interne denticule.

Localités : entre Contlans et lianville, les Clapes; fort late.

(Coll. de il. de Laimbertye.)

Astante ciaressis, Terel. el Jomm, pl. IX, lig. $2 / 4$ el $2 \%$.

A. testa ounta, transversa, in medio el ad umbones inflata, circiler depressa, plicis irregularibus ornatu, lumuln parvula, vix impressa, umbonibus anticis, margine interno indentalo.

Dimensions: Iongueur 39 millim.; hateur 27,5 millim., épaisseur 20,3 mill.

Coquille régulièrment ovale, transrerse, renflée dans le milieu et près des crochets, comprinéc sur le pourtour, ornée de plis concentri(jues irréguliers, lunule très-petite, à peine indiquéc, croelets antérieurs, petits, repliés en dedans, bord intérieur lisse.

Celte espece, par sa disposition transversale, se rapproche de l'A. modiolaris Desh., et s'en éloigne par sa forme régulièremput orale et par son renflement antérieur.

Localités: les Clapes; fort rare.

Cypricardi vitidula, Terq. el Jourd., pl. IX, ligo. 26 el $2 \overline{7}$.

C. lesta ovato-orbiculari, tumida, levigata, striis concentricis, irregularibus, obsoletis ornatu, in medio el al umbones influta, circiter depressa, umbonibus purvis, subanticis, inflexis.

Dimensions: longurur 39 mill.; hateur 34,5 mill.; épaisseur 23 mill.; longueur buceate 2. mill.; longueur anale 3 \& mill.

Coquille ovale, orbiculare, rentlée, lisse et munic de stries et de plis concentriques, obsolètes, irréguliers, renllée dans le milieu et près des crochets, dépriméc sur lout le pourtour; crochets pelits, arrondis, subantérieurs, repliés en dedans ef en arant, lumule et corselet peu sensibles.

Localité : les Clapes; fort rare.

Cymorarda componms, Desh., Deshayes, $T$. élem. de Conch., p. 16, pl. 24, fig. 12-13.

localité Gorze; furl rare.

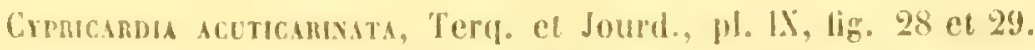

C. testa subtriyonu, transversa, costutis tenerrimis, concentricis, interstitio cquatibus ornata, antico cot infra uscuntu, postice mumenta, subacule angulata, 
area lata, cordiformi, supra concava, infra plana, carina externa acula, interna vix perspicara, plicis obliquis ornata; umbonibus antemedianis, compressis, inflexis, contortis.

Dimensions : longueur 40 millim.; hauteur 30 millim.; épaisseur 23,3 millim.; longueur buccale 24 millim.; longueur anale 33 millim.

Coquille subtrigone, transverse, inéquilatérale, ornée de côtes très-fines, concentriques, égales anx intervalles, avec quelques gros plis d'accroissement trèsespacés; côté antérieu arronidi, côté inférieur arqué, cơté postérieur tronqué obliquement, à angle aiğu, muni d'une large carêne, concave dans le haut, plane dans le bas, limitée par une arête aiguë, carène interne à peine indiquée et ornée de plis obliques, très-fins; bord carlinal arqué, ligament très-court et saillant; crochets antemédians, renflés, comprimés en dessus et contournés en dedans; lunule peu sensible, non circonscrite.

Cette espèce diffère cu $C$. cordiformis, Desh. par l'arête de la carène très-aiguë, surtout près lies crochets, par son côté infériem arqué et par ses ornements.

Localité : les Clapes; fort rare.

Cyprictrdia gregama, Quenst. sp., pl. 1X, fig. 30, 31 et 32.

Isocirdu musa, Sow. Quenstedt, Jur p. 389, pl. LIII, fig. ?2.

C. Lesta minima, subquadrangulari, levigata, jostice truncall, carinata, antice rolundata, supra cxcuvala, umbonibus antemedianis, inflatis, antrorsum incurvis, lunula cordiformi.

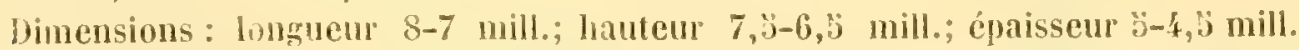

Coquille très-petite, stbquadrangulaire, lisse, tronquée el carénéc en arrière, arrondie en avant et excavée dans le haut; crochets antemédians, rentlés, recourbés en avant, lunule cordiforme, peu profonde.

Quenstedt, ayant confondu cette espèce avee l'\% minima, Sow., n'en donne pas de descriplion; elle en diffère par ses erocheis beaucoup moins élevés et une lumule moins profonde; sa tailfe est at moins quatre fois plus petite, d'apres les dessins de sonwerby et les échantillons que nous possédons de l'I. minima, dont plusieurs sont même plus grands que l'\% tenera, sow.

Cette espèce, généralement fort rare, se trouve parfois réunie en quantité innombrable el se présente dans des conditions semblables dans le Wurtemberg.

Locatité : entre la Sauvage et Differdange, zone du calcaire de Jaumont. (Communiqué par H. de Promorel.)

Cimmita pucicosta, Terq. el Jourd., pl. X, lig. 2 b et 26.

C. Cesta puranla, transversa, ovall, in medio oblique gibbosa, antice et postice compressu, plicis remotis, gradalis ornala, umbonibus inflatis, contiguis, anticis, margine interno denticulato.

Dimensions d'un moule: longueur 1:, in millim.; Lauteur 10 millim.; épaisseur 10 millim. 


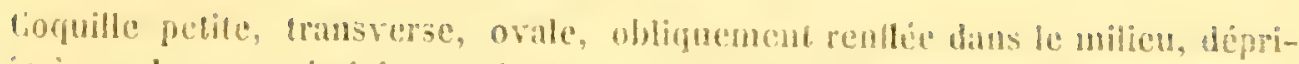
mée à ses deux extrémités; ornée de 1.04 a gros plis concentriques el scalaires; erochets anterieurs, rentles et contigus; bord interne denticuk.

Celle espèce apparticnt à la section des Hippopodiun.

Localité : Longwy (les oflacis); assez rare.

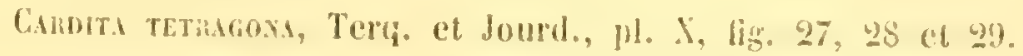

C. lesta ovalo-oblonge, irregulariter tetragonu, costulis tencrimis, concentricis, interstiti minoribus ornata, antice el infre rergulmiter arcula, postice

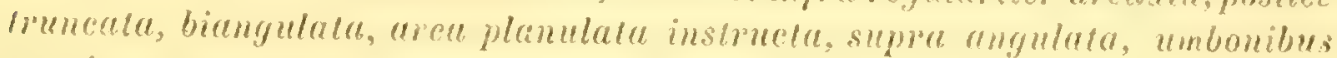
purvis, antemedionis, contignis, lumula angusto, lunceolatu, crelline bidentato. maryine interno cireiler dentulo.

Bimensions: longueur 16-19 millim.; hanteur 12,3-1; millim.; épaisseur 1) $-10,3$ millim.; longueur buecale 9-11 millim.; longueur anale $13, ;-16$ millim.

Coquille orale-oblongue, irrégulièrement lütragone, ornće de côtes très-fines, concentriques, plus petites que les intervalles et interrompues par quelques gros plis d'aceroissement; arquée régulièrememt en arant et dans le bas, tronguée à angle oblus en arrièe et munie d'un corselet déprimé, anguleuse dans le haut et plus oblique en avant qu'en arrière; crochets petits, antemédians, contigus, lunule ćtroite, lancéolée, charnière forméc de deux dents cardinales, bord interne muni de dents sur tout son plourtour.

Loealice : les Clapes: fort rare.

Gexre Lecin, Lamareh.

Le gisement des clapes mésente des especes très-rariées, depuis la forme lenticulaire la phus comprimée jusqu’i la forme renflée, presque spherique; les crochets stivent la mème progression et deviement très-saillants et contournés comme dans les Cypricardes, bien que les efurnières présentent pour toutes les cspeces constamment les mêmes caractères et la mème constilution. Possédant pou chacune de ces especes la chamière farfatement bien conservéc ou mème des coquilles vides, nous voyons que, pour toutes, clle se compose sur la valre droite, d'une dent cardinale oblique, d'une deni allongée latérale antérieure, e! b'une autre posterieure allongée; sur la valre gauche de deux dents cardinales jućgales, d'ure dent postérieure tris-allongée, el d'une autre lamelleuse allongéce antérieure; la lunule est généralement très-étroite, lancéolée et le corselet peu apparent.

lne espece est sans dent et se mondre ideutigue it celle qui est si abondante i) bayeux.

Lucira peregara, Terd. el Jourd., Pl. $\mathrm{X}$, fig. 1 el $\$$.

L. lesta tumida, inayuilaterali, antice quem postice majore, plicis lemellosis

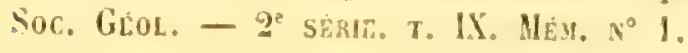


irregulavibus ornate, infra ct antice subcompresse, umbonibus infatis, inflexis, parmper postmedianis, lunula non perspicua, apere comdinali clongata, angusta, curdine edentulo.

bincnsions : longueur 38 milim.; hateur 82 millim. ; éprisseur 26 nillim.; longueur buceale 32 millim.; longueur anale 28 millim.

Coquilie inéquilatérale, le côté antéricur un pen plus strand que le postéricur, rentlée, stobuleuse en dessus, légèrement dépriméc dans le bas et en avant, ornéc te plis lamelleux, irréguliers; crochets renllés, repliés en dedans, un peu postmidians; lunule non marapee, corselet allongé, étroit; charnière suns tents, sous les crochets une petite exearation limite pap une nervure transversale.

Presque tougours me des valves a glissé et vient cheraueheg sur lautre. disposition analogue i celles des Corbules, mais le caractere constamment éfuivalre de la corailie empeche toute confusion.

La pelite excavation yui se remargie sur te bord eardinal interne de chaque valve pernet d'admette que le ligament dépasce l'extromite des nymphes et s'introduit dans l'intérieur de la corquille, ainsi que cela a lieu pour beancoup d'autres genres.

Une coquilie tres-abondante ib bayx et que nous ne trousons pas mentionné dans le prodrome, semble se rapporter is cethe espice el n'en differe que par sa taille environ deux fois plus pehte.

Localité: les Clapes; très-commun.

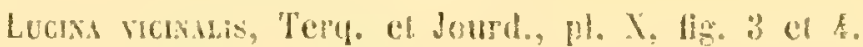

Vexchtes alderse, guenstedt, Jura, p. 360, p1. 48, 1. 26.

L. Iesta crassu, oveta, convexa, plicis irreguluribus ornatu, antice compressa, stanuluta, postice subinflate, umbonibus purvis, antice inflexis, antemedianis, Lunula parva, impressa.

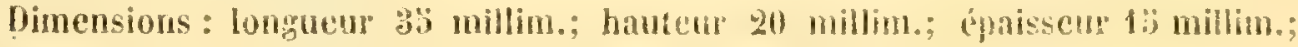
longueur buceale 20 millim.; longueur anale 20 millim.

Coquille epaisse, ovale, convexe, transverse, orné de plis irréruliers, comprinée et conme étalée en avant, renflée et unnie d'un corselet pes sensible en arrière; crochets petits, infléchis en avant, antemédians, lunule iris-petite, non cireonserite.

Cetle espèce diffère des $L$. ovalis et Lo erieina nar sa forme renthe et par son crochet antemedian.

Localite: les Chapes; fort rare.

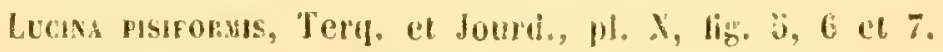

L. testa obovata, pisiformi, subtilissime et concentrice striatu, postice subimpressa, umbonibus medianis, antrorsum infiexis, hunla orbiculari.

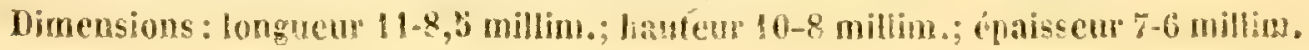


Connilic obovale, fisiforme, orme de stries concentrignes ires-lines el tres-

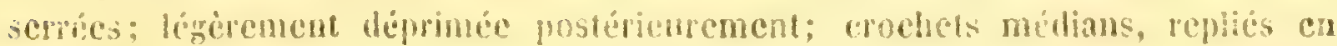
aram, lunule orbiculaire.

l.ocalilé : les Clapes; asse\% rare.

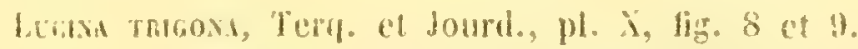

L. tosta lriangulari, angulis oblusis, cuncentrice el sublitissime striata, unbonibus medianis, antrorsum inflexis, antice excevatis, lunulu cordiformi.

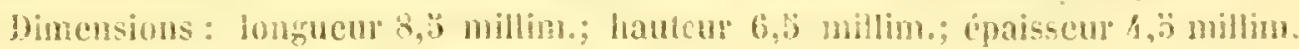

Conuille triangulaire, it angies arondis, ornce de stries extremenent fines et serres et de plis irréruliers, crochets médians rentles, intledis en arant, un peu excavés sur la parte antericure, lunule cordilorme.

Celte espece gédique dans Vinterienr, est la seule dont nous ne possédions

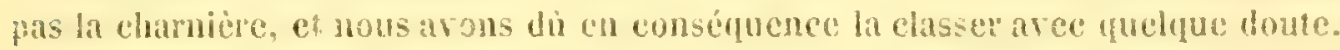

focenlite : les tilipes; fort rare.

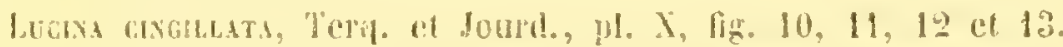

1. testa suborbiculari, sublransvera, circiler compressu, concontrice stricle strulu, latere curdinali uringue obliqua, umbonibus uncmediunis, inflatis, prominentibus, antrorsum inflexis, lunule non porspicus.

Dinensions: longuenr 19-17, mill.; hateur 18-16 mill.; ćpissem 133-11 mil.

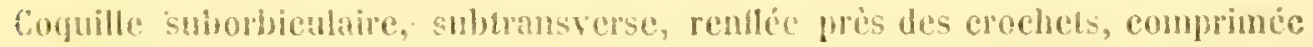
sur le pourtour; ornce de stries très-lines el screées, erochels antemédians, apnllés, élerés et replićs en avant; lunule non matquée, bord cardinal oblique des deux cotés, un peu zlus élaté eu arrièce qu'cn avant.

lne varété est plus orbiculare, a ses crochets nédians et une moindre obliquité dans la partie antérieure du bord eardimal.

Localité: les Clapes; très-commm.

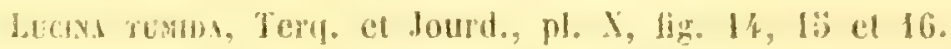

1. Icsta oveto-orbiculari. tumidn, stris concentricis stricte et plicis irregu-

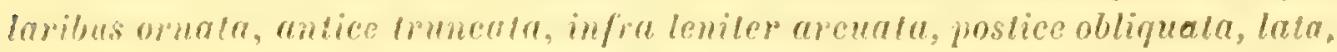
umbomibus infletis, prominentibns, inflexis, antemedianis, lumula non perspirnen.

Dimensions : longueur 20 millim.; hateur 20 millm.; épaisseur 16 millim.

Coquille ovale-orbiculaire, très-renfe, orné de stries concentriques serrées ct de plis irréguliers; légèrement arquée dans le bas, oblique et élargie en arrière, tronquée en avant; eroehets rennes, saillants, replićs en dedans, lunule non indiquée.

Cette espèce, par sa forne irès-ienllée el suborbiculaire, par ses croehels élevés, se rapprocite des Isocades et s'en distingue par les dispositions de la charniere.

Loealité : les Clapes; assez rare. 
Lucisa misconesa, Terq. et Jourd., pl. X, fig. 17 of 18.

L. testa discoiden, compressu, semilunari, lavigata, umbanibus mimimin, medianis, lumula angustissima, lanceolata.

Dimensions : longueur 23 milim.; hauteur 21 millim.; ćpaisseur 8,3 millim.

Coquille comprimée dans son pourtour, semilunaire, discoïde, lisse; crochets très-petits, médians, lunule très-ćtroite, lincéolée, corselet nul, nymphes atreignant le sommet des erochets.

Localité : les Clapes; fort rare.

Lecixs ericix, Terq. ef Jourd., pl. X, fig. 19 et 20.

L. lesla compressa, arbirulasi, striis confertis, irregularibus ornatu, antice sinuata, umbonibus parvis, antemedianis, lunula minima, area plana, vix perspicule.

Dimensions: Iongueu $17,3-16,5-17$ millim.; hateur $17-14,3-16$ millin.; épaisseur 9-7-9 millim.

Coquille comprimée, orbiculaire, ornée de stries irrégulières et serrées; sinueuse près des crochets, comme étalée en arrière et munic d'un corselet plat peu sensible, crochets un peu antemédians, petits, infléchis, lunule très-p̧etire.

Localité : les Clapes; très-commun.

lucixs squanosa, Terr. el dourd, pl. X, lizo. 21 et 22.

L. Iesta compressa, orbiculari, plicis squmosis, concentricis, ivregularibus ornata, umbonibus parvis, medianis, antice inflexis, lunula parva, profunda, cordiformi.

Dimensions : longueur 22 millim. ; largeur 20 millim. ; ćpaisseur 11 millim.

Coquille comprimée, orbiculaire, ornéc de plis concentriques, écailleux, irriguliers; crochets petits, médians, intlechis en avant; lunule petite, profonde. cordiforme.

Cette espece se distingue par sa forme orbiculaire et ses plis écailleux.

Localité: les Clapes; fort rare.

Lecixs ovalis, Terig. el dourd., pl. X, fig. 23 el 24.

L. testa tenue, fragili, compressa, ovata, subaguilaterali, levigata, mitida, idque plicis obscuris instructa, umbonibus parvis, antemedianis, lunula atgustissima, lanceolata, area magna, planulata.

Dimensions : longueur 46-38 millim.; hauteur 3\%-29 millim.; épaissenr 113 milt.; longueur buccale $29-23$ millim.; longueur anale $330-29,5$ millim.

Coquille très-mince, fragile, comprimée, ovale, subéquilatérale, lisse, brillante; ornée de plis espacés ef peu saillants, corselet large, plan, lunule très-étroite. lancéolé, crochets petits, antemédians.

Localité : les Clapes; assez commun. 


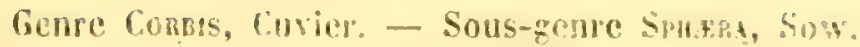

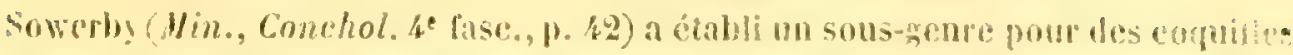
qui, par len charnière, sont identiques aux Corbis et qui s'en éloignent par les".

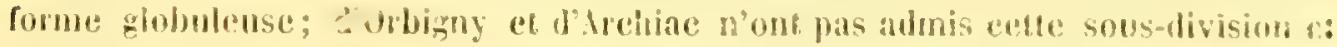

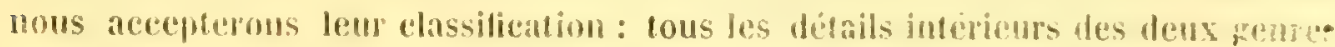
élant identigues, la forme ne sammit constituer un earactère suffisant pour at creation d'un werre, et d’antant plus que nous possedons une coquille qui n'est pas globulease, et qui se rapproche, quani an peste, d'un fossile qui próschle is Posme des Spherese.

l.es Carditu problematien Bur. (Buvignier, Stutistigue prnliontolonigue in

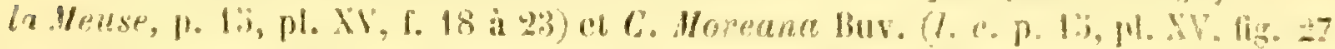

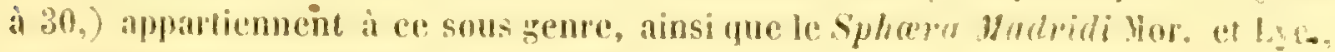

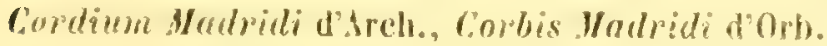

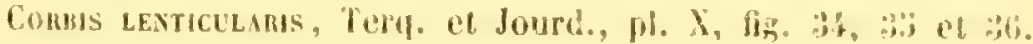

6. Lesta parvu, compressa, lenticulari, plicis irreguluribus, conrentries ornata, infia leniter arcunti, depressu, postice subtrmerta, area pana

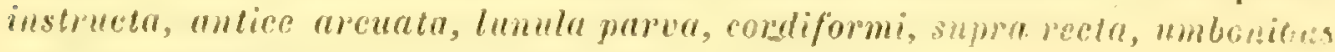
parvis, antemedianis, margine interno denticulate.

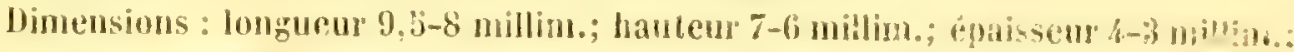
long!nenr bur : i-4, '5 millim.; longueur anale 9-7 millim.

Coquille coute, compriméc, lenticulaire, ornes de ylis roncentrignes incentlieps, légèement arquce ol comprimée dans le bas, trongué el numie d'ma carenc plane en arriere, arondic et munic d'une petite lunule cordiforme oss ayant, droite en dessus; crochels petits antemedians, charniére munie de petids

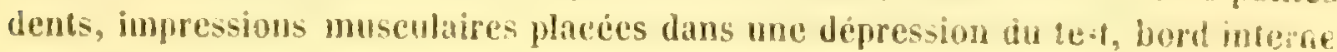
denticule.

Gette espece se distinguc du $C$. oviformis, par sa forme tris-comprimée, pa" ca carene micux marquéc et par les détails intérieurs beauceup moins saillants.

Localité : les Chapes; assez commun.

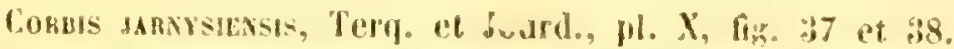

C. testa ovata, subcompressa, supra tenuibus strits concentricis, postis plicis sculariformibus ornata, infra areuata, anticn et postice rolundus. ilque carina obsolela instructa, umbonibus parvis, antemelinnis, lurisu parea, cordiformi, margine interno denticulato.

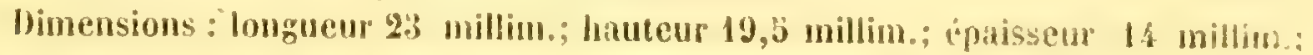
longueur buceale 18 millim, longueur anale 20 millim.

Coquilte ovale, sucomprimce, orné dans le haut de stries concentrijues, ticserrés, dans le bas le plis scalariformes; arquéc dans be bas, arrombic eni avar ef en arricite, munie dune carene peu marquée, crochets petits, antembiars: Immule pefice, cordiforme, bord interienr denticulé. 
Cetce espece, par sa forme eomprimée, se rapprocise du $C$. lenticularis, el m dinère par ses ornements.

Localites : enure Jarny et Contlans; fort rare.

Conts ovwonts, Terq. et Jourd., $11 . x$, fig.

C. lesta parva, cressa, ovelo-ylobulosa, plicis iregularibus, scalariarmi-

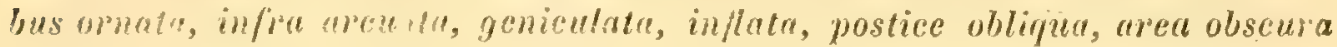
instrueti, mintice areutu, anguste lusulata, supra ungulata, umbonibus porvis, andemedimis, margine interno denticulato.

bimensions: longueu $7-3$ millin.; hateur 10 millim.; ćpaisseur 7 millin.; longueur tuecate 7 millin.; longusar anale 8,5 millin.

Coquille épaisse, courte, olobuletse, ovale, ornéc de plis corcentriques irriguliers, scalariformes; légèrement arquéc, rentlée el genouillée dans le bas, obligue et munie d"un corselet à peine indiqué en arrière, arrondie avec une lumble tres étroi!e en avan! crochets antemédians, petits, impressions museulaires trèssaillantes et portées sur un renforcenent du test, bord intérieur denticué.

Cette cspece se fut remarquer par la disposition genonillée de la partie inférieure, caractere qui se maintient dans tous nos cehantillons.

Le test est trés-épais el tous les détails intérieurs sont très-saillants; la charnière e les impressions musculaires surtout présentent des sallies, fu'on ne reneontre que fort rarement, meme dans les coruilles de gramle dimension.

Localite: les Chapes; tres-commun.

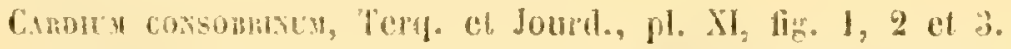

C. teste orbiculari, convexa, juenile costulis regularibus, adulle plicis confertis omata, antice sulco angusto levirer impressu, postice costis ratiantibus sulcatis mimula, unbonibus crassis, medianis, contiguis.

Dimensions: longuour: 31 millin.; hauteur 30 millim, épasseur g2 millm.

Coquille orbiculaire, "rentice, ornée dans le jeune àge de fines côtes concentriques, et dans l'adulte de plis très-serrés, munie en arant d'un étroit sillon, peu sersible, et en arrière d'un corselet orm de grosses côtes tayomantes; crochets métlians, rentlés, contigus.

Cette espece a la forme du L. hillanum, Sow. (Sow erby, Min. Conch., p. 1, pl. 14,

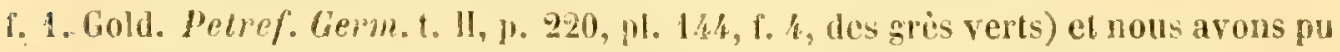
en prendre la deseription latine presque cu son cntier; elle en diffère par son corselet plus étroit el par ses omements qui ue se mantiennent pas identiques a tous les âges de la coquille.

Localite: les Chapes; fort are.

Combur cogsares, phil.

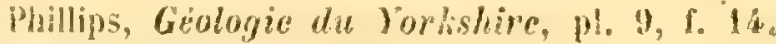

localite : hongwy (glacis); assez commun. 


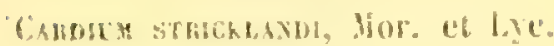

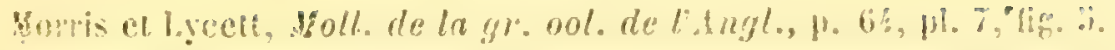

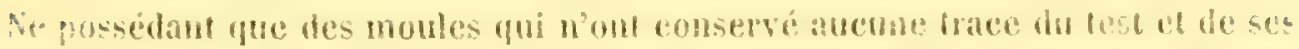

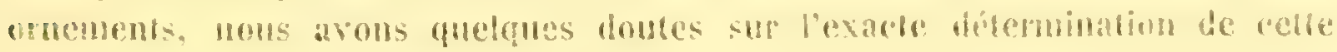
espece.

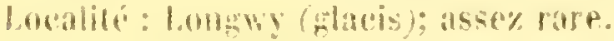

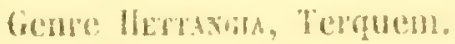

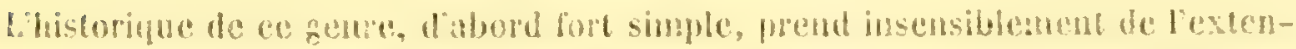

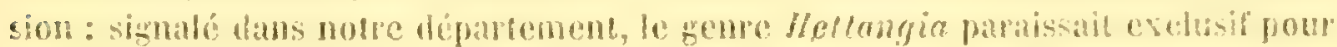

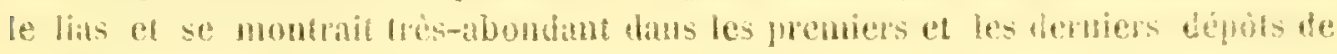
cello: formation.

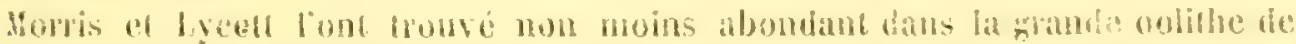

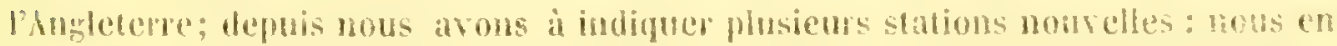

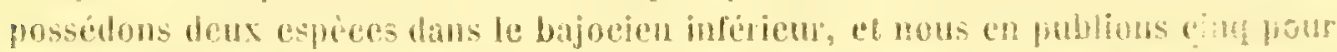

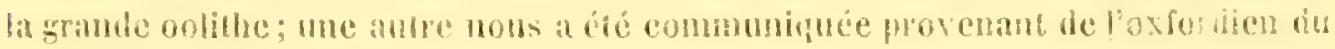
(aip) de la lleve.

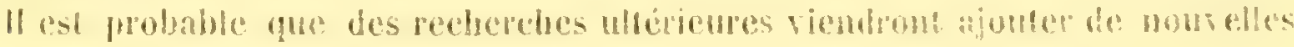
donness sub liextension de ce genre.

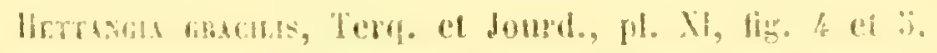

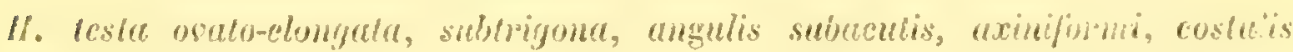

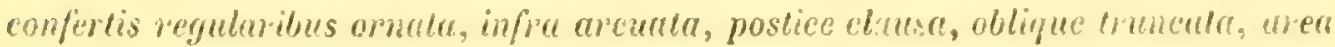
plana, plicis oblaquis ornata, acule carinatu, antice subpraducta, compresin, pumbonibus postmedianis.

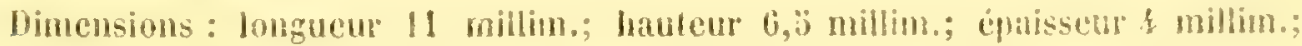

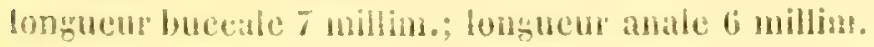

Conuille osale-sllongéc, subtrigone, à angles inféricurs subaigus, osnéc de fincs côtes trés-serrées et régulibres, arquée dans le bas, oblique, mume d’u corscię

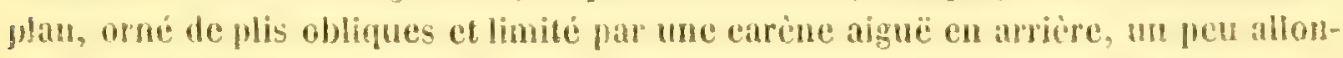
gée ei compriméc en itrant; crochets petits, repliés en dślans, postmédians.

Localite: les Clapes; fort rare.

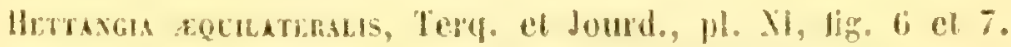

H. testu ovalo-oblonga, aquiluterati, infra subarcuata, postire clausa, obliqua, area angusta, carina obtusu, untie depressa, angulo rolundato, umbonibus previs srieclianis.

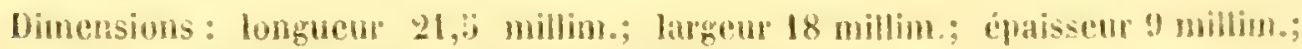
longueur buecale Is millim, lon:zeur anale 19 millim.

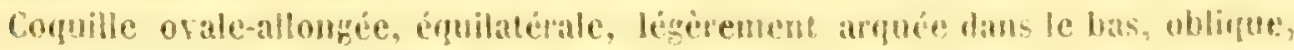




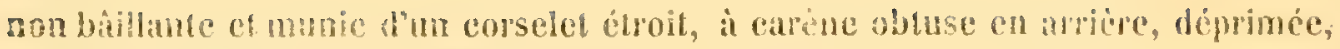
ângle arrondi en avan, eroẹcts pelits, médians.

Relle espèce, assez mal conservée quant à sa suface externe, présente l'interne avec une netleti remarjuable, et démontre que limpression palléale ne posside pas de sinus, comme l'indiquent Quenstedt et l'ielet.

sorealite: les chares: fort rare.

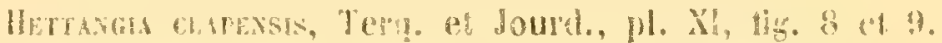

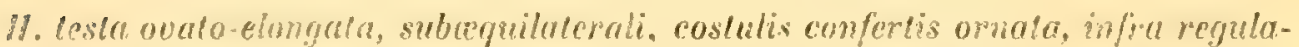
viem arculue, postice clausu, obliqun, aren angustu, angululc, plicis minuta, caring obluse, limbatr, antice allemata, angulo rotundato, umbonibus parvis sulsmedionis.

Dimensions : longueur 32 millim.; hauteur 18 millim, épatiscur 10 millim.; Lomanur buecale 18,3 millim, Iongueur anale 20 millim.

Connille orab-allongee, subéquilatérale, ornée de fines còles concentiques, ségnlièrement arqqué dans le bas, obiche en arrère, mune d"un corselet angu-

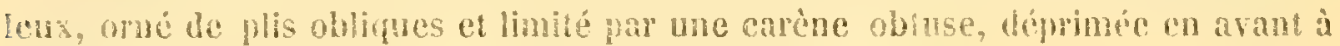
angle arrondi, crochets pelits un pers antemédians.

folte espoce se distinge par un caractere jue ne posselde ancme aure: la rantic postéreure est un peu jlus grande que l'antérieure.

Localite : les Clapes; assez commun.

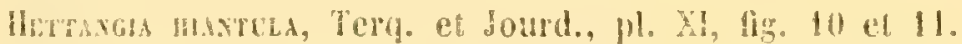

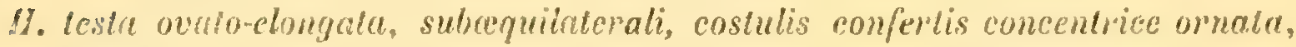

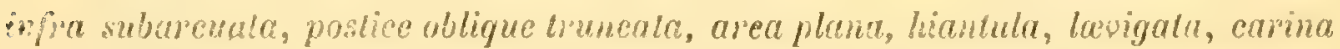
cbscura, anice producta, angalo rotundalo. mergine cardinali vecto, umbonibus provis, medianis

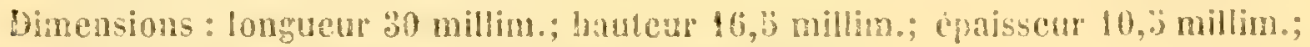
Longueur bucale 18 millim.; longueur anale 18 millin.

Coquille ovale-allongée, subéquilatérale, ornéc de fines côtes très-serrées, fres-légèrenent arquéc dans le bas, oblique ef tronguée en arrière, munie d'un corselet large, ylat é lisse, limité par une carène peu sensible, doté d'un bâillement liuéaire, déprimée el arrondie en avant; bord cardinal droit, angle postérieur ués-marqué, crochets petits, médians.

Celle espèce est la seule du systène oolihique g̨ui se montre mumic dua Lithement postéricur.

hosalité : les Clapes; fort rare.

lietrangla subangulars, Terq. el Jomrd. pl. Xl, lig. 12.

11. Icsia ovato-oblonga, subtrigona, angulis rotundatis, costulis confertis reguLariter ornate; infra lemiler areuala, postice clausa, truncala, brevi, aren plana. 
lavigata, carena oblusa, antice producta, altenunta, umbonibus purvis, inflexis, in terlia postica parte sitis.

Dimensions: longueur 27 mill.; hauteur 18 mill.; épaisseur '9 millim.; longueur buceale 20 millim.; longueur anale 16.5 millim.

Coquille ovale-allongée, subtrigone, à angles inférieurs arrondis, ornée de fines côtes concentriques, très-serrées, légèrement arquée dans le bas, tronquée, courte, munie d'un eorselet lisse et limité par une carène obtuse en arrière, allongée et retrécie en avant; crochets petits, infléchis et placés aux deux tiers anterieurs.

Cette espèce diflère de 1'Il. gracilis par ses angles obtus, par la position des crochets et par son corselet presque antérieur.

Localité : les Ciapes; fort lare.

Isocardia gibbosa, Mtu.

Nunster in Goldfuss, Petref. Germ., t. 1I, p. 209, pl. 140, 19. 10.

Cette coquille, aussi épaisse que haute, est ornéc de côtes verticales régulières et très-scrées et de stries transversales très-fines, qui cotwrent toute la surface de croisillons.

Localite: Ies Clapes; assez rare.

Isocanda siNiMa, Sow.

Sowerby, Min. Conch., n. 334, pl. 29:3, tig. 1-3̈.

Localité : Gravelotte (marnes); assez commun.

Isocardia texera, Sow.

Sowerby, Min. Conch., p. 334, pl, 295, fig. 4-6.

Localité: Gravelotte (marnes); assez commun.

Isocardia rostrata, Sow.

Sowerby, Min. Conch., p. 334, pl. 293 , fig. $7-8$.

Localité : Gravelotte; assez rare.

Isocardia clapersis, Terig. et Jouäd., pl. Xl, fig. 13 et 14.

1. testa ovata, tumida, subaquilaterali, cortliformi, striis conferlis, regularibus ornata, infra compressa, circiter arcuata, postice area ncute prominente, sinu limitata, oblique plicata munila, umbonibus rotundatis, inflexis, medianis, margine interno lovigato, lunula parva.

Dimensions : longueur 22, 3 millim.; hauteur 23 millim.; épaisscur 17 millim.

Coquille ovale, renflée dans le haut, très-comprimée et arrondie sur son pourtour, subéquilatérale, ornée de fines stries concentriques très-serrées,

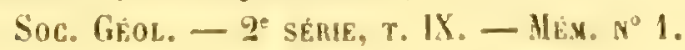


corselet cordiforme, bombé, limité par un sillon, orné de plis obliques et occupant toute la hauteur de la coquille; crochets petits, renflés, repliés en dedans, lunule petite, non circonscrite, bord interne lisse.

Cette espèce diffère tour et par la longueur du corselet.

Localité : les Clapes; fort rare.

Isocardia bullata, Terq. et Jourd., pl. XI, fig. 1 b̈ et 16.

1. tesla ovata, tumida, subaquilaterali, plicis concentricis irregularibus ornata, circiter rotundata, area acute prominente, cordiformi, dimidiam lateris partem oblegente, sinu limitata, umbonibus medianis, rotundatis, antrorsum inflexis, lunula parva, orbiculari, margine interno lavigato.

Dimensions : longueur 22 millim.; hauteur 28 millim.; épaisseur 20 millin.

Coquille ovale, renilée, subćquilatérale, arrondie sur son pourtour et légèrement comprimée sur le bord, ornée de stries concentriques très-fines, trèsserrées dans le haut, et très-espacées dans le bas; corselet cordiforme, saillant, limité par un sillon et occupant environ la moitié de la hauteur de la coquille; crochets élevés, renflés, repliés en dedans et en avant; lunule petile, peu profonde, arrondie; bord interne lisse.

Localité : les Clapes; fort rare.

Nugula Nodiferi, Terq. et Jourd., pl. XI, fig. 17 et 18.

$N$. testa ovata, inflata, rotundata, transversa, striis obscuris plicisque ornata, infra irregulariler arcuata, idque sensim antice sinuata, postice producta, area 6 secessis nodulis munitu, umbonibus antemedianis, hnula paroa.

Dimensions : longueur 19 millim.; hauteur 12 millin.; épaisseur 9 millim.

Coquille ovale, renflée, transverse, arrondie, ornée de stries concentriques obsolètes et de quelques plis plus marqués, irrégulièrement arquée et un peu sinueuse inférieurement, allongée postéricurement en forme de coin, munie d'un corselet peu prononcé ct orné de 6 petits nouds espacés, décurrents du sommet des croehets; crochets petits, antemédians; lunule peu sensible.

Localité : entre Jarny el Conflans; assez rare.

Nucula digora, Terq. et Jourd., pl. XI, fig. 21 et 22.

$N$. testa minima, compressa, lcevigata, antice truncata, digona, postice spatu. lata, umbonibus anticis.

Dimensions : longueur 2,3 millim.; hauteur $1,3 \mathrm{millim}$.

Coquille très-petite, comprimée, lisse, tronquée et digone en avant, étaléc et arrondie en arrière, crochets antéricurs.

Localite : Fontoy, $6^{\circ}$ couche ; fort rare. 
Necula lateralis, Terq. el Jourd., pl. XI, lig. 19 et 90.

N. testa ovata, circiter compressa, lavigata, infra arcuata, postice productu, antice breve, lunula cordiformi, clongata, umbonibus parvis, sibanlicis.

Dimensions : longueur 10-3 millim.; hauteur 7-4 millim.; épaisseur 4,30 -3 mill.; longueur buceale $3-2$ millim.; longueur anale $8-4$ millim.

Coquille ovale, comprimée sur son pourtour, lisse et brillante, arquée inférielrement, allongée en arrière, courte en avant; lunule cordiforne, occupant toute la surface antéricure, crochęs petits, subantéricurs.

Localité : Ies Clapes; assez commun.

Nuculs equllateralis, Terq. et Jourd., pI. XI, fig. 25, 24 et 25.

$N$. testa ovata, compressa, lavigata, aquiluterali, infra leniter arcuata, supra angulosa, umbonibus minimis, medianis.

Dimensions: longueur 9-8,5-8 millim.; hauteur 6-6, $6-6$ millim.; épaisseur \&-1-4 millin.

Coquille ovale, comprimée, lisse, équilatérale, doucement arquée dans le bas, anguleuse en dessus; crochets très-petits, médians.

Deux petites nervures partent du sommet des crochets en arriere et en avant; elles simulent un corselet et une lunule lancéolés, exactement de mème forme et de mème dimension, de la sorte il est impossible de distinguer la partic antéricure de la postérieure.

Localité : les Clapes; issez commun.

Nuculas vexusta, Terq. et Jourd., pl. XI, fig. 26, 27 et 28.

$N$. testa ovata, transversu, inflata, rotundala, lavigata, antice brevi, vecta, infra oblique arcuata, postice altenuata, supra recta, umbonibus anticis, inflatis, inflexis, lunula magna, cordiformi, area angusta, clongata.

Dimensions : longueu 18 ö millim.; hauteur 10 millim.; épaisseur 9 millim.; longueur buceale 8 millim.; longueur anale 12 millim.

Coquille ovale, transverse, renthé, arrondie, lisse, tris-courte, verticale en avant, obliquement arquée dans le bas, rétrécie en arrière, droite en dessus; munie d'une lunule large, cordiforme, occupant toute la face antéricure, et d'un corselet étroit et allonge; crochets renflés, repliés en dedans, chamière formée de dents sériales nombreuses ef très-fines.

Cette espèce se distingue du $N$. nucleus, Desl. (Deslongchamps, Hém. de la Soc. linn. de Norm., t. VI, 1838. Coquilles de la piorre blanche de Cacn, p. 71, pl. 1, ligo. 8), par une forme plus élargie et moins rentlée, et surtout par les dimensions de la lunule.

Localité : les Clapes; très-commun. 
Leda lacryma, Sow. sp.

Nucula lachyia, Sowerby, Min. Conch., p. 492, pl. 476, fig. 4-6.

Cette espèce est lisse et diffère un peu de celle de Sowerby par sa carène profonde et très-prononcée.

Localité : les Clapes; assez commun.

Genre Trigonis, Bruguière.

Dans son traité élémentaire de Conchyliologie, H. Deshayes fail remarquer combien la constitution des coquilles vivantes diffère de celle des coquilles fossiles.

Les premières ont leurs côtes rayonnantes et écailleuses, leur bord intéricur dentelé, le corseleı peu marqué; leur ensemble externe présente toute l'apparence d'un Cardium.

Dans les secondes, les côtes sont plus ou moins transversales et en aucun cas rayonnantes; le bord interne est lisse ou marqué de quelques plis en forme de zrouttières; le corselet, toujours apparent, est le plus souvent divisé en trois parlies, séparées par des carènes élevées.

M. Deshayes ajoute : "Dans les espèces vivantes nous n'apercevons aucune trace de l'épiderme velu et caduc que l'on observe fréquemment dans les autres familles des lamellibranches.

Ce qu'un observateur aussi profond n'a pu reconnaitre sur les coquilles vivantes, on devait avoir peu de chances de le trouver sur des coquilles fossiles, et cependant nous eroyons l'avoir rencontré, quoique indirectement, sur plnsieurs de nos espèces.

Nous attribuons à cet épiderme, que la fossilisation n'a pu conserver, la production sur la surface du T. lineolata d'un réseau très-serré el régulier, formé de petites sphères disposées suivant des lignes qui se coupent à angle droit; il en est de mème pour le $T$. costellata.

Sur le $T$. clapensis, ce réseau est moins régulier et formé par des lignes qui se croisent sous un angle très-aigu.

Ce réseau couvre toute la surface de la coquille, y compris le corselet et contourne les côtes.

Trigonis producta, Terq. et Jourd., pl. Xl, liğ. 29 et 30.

T. testa trapezoidea, compressa, retrorsum producta, aliformi, antice et infra arcuala, supra angulata, latere costis nodosis arcuatis ornata, non lunulata, area tripartita, duabus exlornis carina nodulosa, idque a nodis duabus vel tribus costulis obliquis instruetis, area internu lavigata, umbonibus subanticis.

Dimensions : longueur 26 millim.; largeur 18 millim.; épaisseur 8,5 millim. 
Cuquille trapézoìdale, comprimée, allongée en forme d'aile, arquée en avant, arrondie, puis sinueuse dans le bas, tronquée en arriere, très-oblique en dessus; ornée de nodules en séries arquées et régulières; corselet formé de trois earènes, les deux externes séparées par une nervure tuberculeuse, moins élevée que la nervure externe et l'interne; de chaque nervure patent deux ou trois cótes transversales; carène interne lisse; crochets subantérieurs, repliés en arrière.

Cette espece se rapproche par sa forme des $T$. aliformis et caudau, et s'en distingue par les ornements des cótés et surtout bar ceux les carènes.

Localité : les Clapes; fort pure.

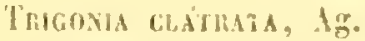

Agassiz, Uim. su' les Trigonies.

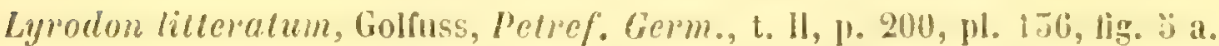

Gỏdluss a réuni sons ce nom plusients esprees que hgassiz a cru dev ir séparer.

Localité : les Clapes; fort rare.

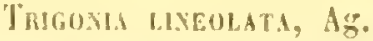

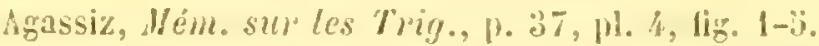

Cetle espece, dans un éta de conservation admirable, présente bien le caractère spécifque que lui attribue Agassi\%: le corselet est lage et les carènes sont uniformément réticulées; cette partie de la corpuille étant d'une grande nelteté. nous a paru mériter d'ête lessince à noureau, d'autant plus que nous avons à représenter le réseau épidernique.

Localite: les Clapes; commun.

TrigoNiA COSTATA, Lmk.

Lamarck, Hist. nut., Vi, p. 64. Enc. mél., pl. 28:8, fig. 2.

Agassiz, Hém. sur les Trig.

Sowerby, pour le $T$. elongata, fail observer avec raison que dans ce genre, les coquilles sont très-variables et qu'on peut considérer cette espèce comme me variété duT. costata.

Agassiz (Hém. sur les Triy., p. 46), pour le T. cardissa, dont les ćchantillons lui ont été communiqués du Musće de Bâle, sans indication de terrain, pense qu'ils provionnent du grès vert, ì en juger d'après leur apparence et leur bel état de conservation.

"Il ne faut pas confondre, ajoute Agrasiz, ecte espece avec le T. elongata, Sow., qui se trouve dans les terrains jurassiques de l'Angleterre; cependant if serait possible que la figure 3 de Sowerby (les $n^{\circ} 1$ et 2 des grès verts des environs de Weymouth) que cet auteur dit provenir de France, fût un T. curdissa. ๖

Les Trigonies que nous possédons, bien que provenant d'un seul et même 
terrain, varient beaucoup, quant à leur coloration, selon la constitution de la roche qui les renferme : pétrifiées par du calcaire spathique, rouge-jaunâtre aux Clapes, elles sont grises à Gorze et à Amanvillers, et d'un noir-bleuâtre dans les marnes de Fontoy, de Giavclotte et de Longwy.

Quant à leur forme résultant de leurs dimensions, nous donnons pour 4 échantillons les rapports de la largeur du corselet arec celle de la partie costellée, prise à l'extrémité de la carene interne, et nous démontrons ainsi que le T. cardissı arrive au costata en passant par l'elongata, les ornements restant à très-peu près identiques, pour le nombre et la disposition des côtes et la forme du corselet.

Largeur du corselet. . . . . . . . . 17-17-20-20-13 (1)

Laryeur de la partic costellée. . . . . 21-27-32-34-15

Hauteur de la coquille. . . . . . . 42-45-5:3-57-43

Localités : Longwy (glacis), très-commun; les Clapes: Fontoy, Grarelolte (marnes); fort rare.

Trigona clapexsts, Terq. et Jourd., pl. Xl, lig. 31,92 et 33.

T. testa trapezoidea, antice arcunta, infra rotundata, postice truneata, supre obliquata, juvenile costis arcuatis, tuberculosis, alulte luberculis interne obliquis, externe transversalibus, in medio irregulavibus ornata; "rea tripartita, suprn costata, postice plicis irregularibus muitu, carina interna parcinodulosa, alteri paululum nodulost, sinu nolatu, tortia nodosa; umbonibus parvis, anticis, inflexis, margine interno duobus latis sulcis instructo.

Dimensions: longueur 38-31, $3-19$ millim, hauteur 29-29,5-13 millim.; epaisseur $20-1 \%-10$ millim.

Coquille trapézoïlale, arquée en avant et munie d'use lumule peu apparente, arrondic dans le bas, tronq̨uée en arrière; dans le jenne âge, ornée de tubereules cn ares réguliers; dans l'adulte et en avant, de tubercules en séries horizontales, en arrière, de séries obliques, el dans le milieu, de tubercules disposés irrégulièrement; corselet divisé en trois parties ormées de côtes transversales dans le haut et de plis sur le reste de leur surface; carène interne marquée de nouds très-espacés; carène moyenne de tubereules très-fins et suivie d'un sillon; carène externe de gros tubercules saillants; crochets subantérieurs, petits, repliés en dedans; bord intérieur muni de deux larges et inégales gouttières.

Comme nous l'avons dit plus haut, cclle espece porte sur sa surfice un tissu à mailles régulières et très-serrées; il est formé par la rencontre de lignes obliques, tandis que dans le $T$. lineolata, ces lignes se coupent suivant un angle droit.

Cette espèce, par la disposition générale de ses ornements, tant des côtés que du corselet, se rapproche du T. litterala Ag. du grès supraliasique; elle s'en distingue par ses côtes transversales qui sont noduleuses et espacées; tandis que (1) $\boldsymbol{T}$. elongala, Sow. $\mathbf{n}^{*} \mathbf{g}$ d'après la figure. $T$. cardisga, Ag. d'aprés la figure. 
dans le T. litterata, elles sont lisses, trés-serrées et commencent à se produire dis le jeune âge.

Localité: les Clapes; très-commun.

l'migona detrita, Terq. el Jourd., pl. Xll, fig. 1 et 2.

T. testutrupczoidea, compressa, antice arcuatu, postice eiongatu, attenuats velut detritu, juvenile lateribus et curinc costis apeuatis mox anyulatis, adulte plicis ornala, xrea magna, tripartitn, enrina interna levigate, duabas externis sulco sepuratis, supra costalis, inferne plicatis, umbonibus anticis, retrorsum inflexis, lunula levigata, elongata.

bimensions: longueur 78 millim.; hauteur 71 millim.; cpaisseur 34 millim.

Coquille trapézoïdale, comprincée, arquéc en avant, allongée et déprimée en arrière, à surface comme usée, onnée dans le jeune âge de còtes al jućes près des erochets, puis anguleuses, et de côtes obliques sur le corselet, ornemonts disparaissant bientôt et remplacés par des plis larges et irréculiers, repliés s les deux carènes externes; carène interne lisse, les deux externes séparées par un sillon, lunule ćtroite, allongée et lisse, crochets antérieurs et repliés en arrière.

L'extrémité des còtes reste visible le long du bord antérieur, et contre le corselet on apereoit de gros tubercules verticaux qui vont rejoindre les plis concen-
triques.

Près des erochets les trois carenes sont nettement séparées, postérieurencus elles se corrfondent.

Localité: les Clapes; fort lare.

Trigomia scifbergexsis, Lyc., pl. Xil, figo. 3 el 4 .

Norris et lycett, Suppl. des Nol. de la gr.ool. de l.Angl., p. 48, pl. 37, fig. 1.

Cette especee, une des plus grandes du grenre, est ornée de gros tubercules près du corselet, et de coites obliques, noduleuses, arques et irrergulieres en arant; caraclères qui servent à la distinguer du $T$. signula, du bajocien inférieur, dont les côtes antéricures sont droites et régulières.

Notre échantillon est incomplet el cassé à son extrémité postérieure; il posséde ces dimensions: hauteur 118 millim.; largeur 108 millim.

Horris et Lyeett indique cette espece dans le Cornbrash du Yorkshire, où elle est assez commune.

Localité: Wohécourt (Yeuse); fort rare (Collection de II. Colliez.).

IsoAica, Munster.

Nous ne possédons de ee genre qu'un moule indéterminable et nous ne le mentionnons que pour compléter les séries; nous sirnalerons la présence de plusieurs 
espèces dans le bajocicn supériour (le calcaire à polypiers) de la Hoselle, et la plupart des fossiles se montrent munis de leur test.

Localité : Longwy (glacis); fort rare.

Cucullées et Mrches.

Il est reconnu que l'organisation de l'animal est la même pour les Cucullées que pour les Arehes, et que la charnière seule differe dans ces deux sortes de coquilles; mais comme les premières sont de beaucoup plus abondantes que les sceondes dans les formations anciennes jusque dans le terrain crélacé, tandis qque l'inverse a lieu pour les terrains tertiaires et l'époque actuelle, nous avons cru devoir mainlenir ces deux divisions; l'une caractérisée par des dents sériales et verlicales, l'autre par des dents plus ou moins allongées et horizontales.

Dans les polypiers des Clapes, l'Isastrea serizhis, le Thamnastrea Defranciana et surtout dans l'Isastrea Conybeari, nous arons trouvé des Arches el des Cucullées placées dans des loges, comme les Gastrochènes, les Saxicaves et Ies Lilhodomes, et exactement de même forme et dans les mêmes condilions que celles qu'on observe dans les Polypiers des rérions équatoriales.

II est à remarquer que ces coquilles, qui habitent l'intéricur des roches, présentent parfois sur les flanes une dépression comme les espèces qui ne se creusent pas de loges et qui sont munies d'un byssus; il arrive même que, dans certaines espèces, cette dépression est plus forte et plus accusée dans les premières que dins les secondes.

Les espèces perforantes ont génératement les erochets rapprochés el l'airc ligamentaire très-étroite el linéaire.

Dans les Cucullées, les longues dents sont linement striées verticalement; ce caractère se présente identique dans les coquilles vivantes comme dans les fossiles.

Contrairement à ce qu'on observe habituellement dans la dispersion des espèces des genres Cueullées et Arches, Morris et Lycett ont reconnu dans la grande oolithe de l'Angleterre 10 Arches et 3 Cucullées; nous avons trouvé dans l'oolithe inférieure de la Noselle 6 Cucullées et 4 Arches.

Arca pectunculotdes, Terq. el Jourd., pl. Xll, lig. 3 et 6.

A. testa fragili, subr'lomboidali, clonguta, compressa, costis radiantibus crebris et transversalibus minutis ornatn, antice rotundatu, infra et sumra recta, postice compressa, producta, anguste chimata, umbonibus parvis, subanticis, approximatis, area ligamenti lineari, cardine dentibus in medio parvis, utrinque crescentibus.

Dimensions : longueur 20,3 millim.; hateur 11 millim.; épaisseur 8 millim.; Iongueur buceale 10 millim.; longueur anale 16 millim.

Coquille très-mince et fragile, subrhomboïlale, allongée, comprimée dans son 
ensemble, ornée de cótes rayonnantes serrées et de stries transversales trísespacées, arrondie en avant, droite dans le haut et le bas, très-comprimée, allongée en arrière et munie d'un corselet étroit à earène peu sensible; crochets petits, subantérieurs, comprimés et rapprochés, champ ligamentaire linéaire: charnière formée de dents sériales très-petiles, s'agrandissant vers les extrémités et disposées comme dans les Pétoncles.

Localité : les Clapes; fort rare.

Aнса sтriat0-pexctata, Terq. el Jourd., pl. Xll, fig. $9,10,11$ et 12.

A. lesta parvula, lrapezoidea, ratundata, corallivora, striis concentricis punctatis ornata, antice aliformi, aliquot costulis radiantibus instructa, infra leniter areuata, in medio compressa, postice truncalt, area magna, curine acuta limilatu, costulis obliquis ornatn, umbonibus antemedianis, inflexis, arca ligamenti anguste rhomboidali.

Dimensions : longueur 10 millim.; Lauteur 7 ıillim.; épaisseur 3 millin.; longueur bucealc 6 millim.; longueur anale 8 millim.

Coquille petite, trapézoïdale, arrondie, ornée de stries coneentriques ponctuées, arrondie, comme ailée et douée de g̨uelques côtes rayonnantes en avant, légèrement arquée dans le bas ef un peu sinueuse dans le milieu, tronquéc en arrière; corselet large, limité par une carène aiguë ct orné de fines cótes ibliques; сrochets antemédians, comprimés, champ du ligament étroit, rhomboüdal, charnière munic de dents sériales.

Nous possédons des coquilles virantes, provenant de la Guadeloupe, qui se temaient dans des polypiers et qui se distinguent trè-dificiement des fossiles; Ia carene est munie de longues épines liexibles.

Localité: les Clapes, dans l'Isastrea serialis; assez rare.

Ancia ixtusplicata, Terl. et Jourd., pl. Xll, fig. 7 el 8.

A. testu parvula, ovato-oblonga, corallivora, costulis radiantibus obtusis et strits transversalibus clathratis ornata, margine cardinali ct inferno paralbelt, antice et postice rotundala, area lata, quinque costata, carina clata, nodifera, umbonibus parvis, antemedianis, inflexis, area ligamentilincari, snurgine interno utrinque plieuto.

Dimensions : Ionğ 12 millim.; hautcur 6,3 millim.; diamctre 5 millim.

Coquille petite, ovale-allongée, ornéc de nombreuses cỏes rayomantes, obtuses, phus grosses en arriere et en avant que dans le milieu de la coquilfe, à intervalles très-étroits, et de stries transversales; bords supérieur et inférieur

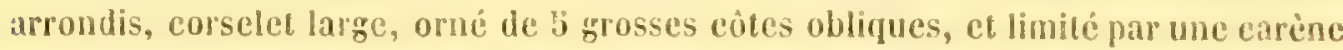
très-élevée, munie de gros lubereules; crochets petits, déprimés, antemédians, aire ligamentaire linéaire, bord interne plissé à ses deux extrémités.

Loealité: Ies Clanes ; fort rare.

SOC. GEOL. - Q SERE. T. IX. NEM. N 1 . 
Arca pulcha, Sow.

Sowerby, Afin. Conch., p. 488, pl. 473, fig. 3.

Cette espece est fabiforme, régulièrement ovale, à champ ligamentaire linéaire ct corselet à peine indiqué.

Nous avons trouvé dans des Bryozoaires et des Polypiers de la Guadeloupe des coquilles qui présentent exactement la même forme; les côtes rayonnantes et la carène sont seulement un peu plus prononcées.

Localité : les Clapes, dans les Isastrea Babeana et serialis; assez rare.

Cucullat oblonga, Sow.

Sowerby, Min. Conch., p. 20u, pl. 206, fig. 1-2. Area oblonga, Goldfuss, Petref. Germ., t. 1I, ९. 147, pl. 123, fig. 2.

Localités: les Clapes; très-commun. Saint-Thiébault près de Gorze; très-rare.

Cacullea surdecussata, Mu. sp.

Area subdecussata, Hunster in Goldfuss. Petref. Germ., t. 11, p. 147, pl. 123, f. 4.

Localités: Ies Clapes; très-commun. - Gravelotte (marnes); asse\% rare.

Longwy (glacis); rare.

Cucullea longata, Suw.

Sowerby, Min. Conch., p. 469, pl. 447, fig. 1 .

Localités : les Clapes; fort rare. Saint-Thićbault près de Gorze; très-abondant.

Cucullea texturata, Mu: sp.

Area texturata, Hunster in Goldfuss, Petref. Germ., t. II, p. 147, pl. 123, f. 3.

Cette espèce étant perforante ou arénicole, ne montre aucune dépression ventrale, a les crochets repliés en dedans et en avant et le champ ligamentaire trèsétroil; caracteres que Goldfuss indique dans le texte allemand, mais qu'on ne saurait reconnaitre sur la figure, la coruille n'étant représentée que par le côté.

Localité : les Clapes; assez commun.

Cucullea ovifonms, Terq. et Jourd., pl. Xil, figg. 13 à 17.

C. testa oviformi, rotundata, striis concentricis et radiantibus confertis, clathratis ornata, umbonibus medianis, inflatis, depressis, antrorsum inflexis, carina vix perspicun, area ligamenti angustissima.

Dimensions : Iongueur 16,3 millim.; hauteur 11,3 millim.; épaisseur 10 millim.

Coquille oviforme, plus haute en avant qu'en arriere, arrondie, ornée de stries concentriques, et de côtes rayonmantes et serrées, couvrant toute la surface de croisillons rézuliers; crochets renflés, médians, déprimés et infléchis en avant; corselet peu apparent, champ ligamentaire trìs-étroit.

Localité : Ies Clapes; assez rare. 
Cucullek famfonmis, Terq. et Jourd., pl. XIt, lig. 18 à 21.

C. besta ovala, infata, rotumlata, fabiformi, corallivora, costulis concentricis et striis radiantibus aqualibus, clathratis ornata, antice et posticc compressa, aliformi, infira recta, in medio subimpressa, area postica vix perspicua, umbonibus submedianis, inflatis, inflexis, area ligamenti linerri.

bimensions: Iongueur 12 millim.; lanteur 8 millim.; épaisseur 6 millim.; longueur buceale 7,5 millim.; Iongueur anale $9,3 \mathrm{~m}$ millim.

Coquille ovale, renflée, arrondie, perforante, ornée de côtes concentriques et de stries rayonnantes érales, arrondic et comprimée en forme d'aile en avant et en arrière, droite dans le bis el marquée d'une légère dépression qui remonte sur les flanes; corselet à peine sensible; crochets submédians, renflés, infléchis, trèsrapprochés, champ du ligament linéaire.

Localité: les Clapes, dans l'Isastrea Babcuna; assez commun.

Pixna cuneata, Phil.

Phillips, Géol. du York, p. 122, pl. 9, fig. 17.

Localités: Longwy (glacis), les Clapes, Gravelotte (mames); partout assez. commun.

Mrruus ghaditus, Terq. el Jourd., pl. Xil, fig. 22, 23 el 24.

M. Lesta ovato-elongata, transversim rotundata, costulis raliantibus strictis, bifurcatis, et aliis transversalibus, gradatis, clathratis eleganter ornatu, dorso convexa, ventro excruata, antice el postice inflata, rolundata.

Dimensions : longueur 43 ụillim.; largeur 21 millim.; épaisseur 20 millim.

Cofuille ovalc-allongée, transversalement arondie, ornée de côtes rayonnantes, arrondies, très-scrrées, se bifurquant suecessivement avec la croissance de la coquille et d'autres plus grosses transversales, en gradins espacés, déterminant des croisillons avec les premières; convexe sur le dos, concave aux deux tiers de la hauteur ventrale, renthée et arrondie à ses deux extrémités.

Localité : les Clapes; asscz rare.

Mrtilus imbricatus, Sow. sp.

Modiola imbricata, Sowerby, Hin. Conch., p. 262, pl. 212, fig. 1-2.

Morris et Lycett (Hol: de la gr.ool. de l'Angl., p. 41, pl. 4, fig. 19) indiquent cette espèce pour plusieurs localités de la grande oolithe et dans les conches qui lui sont supérieures, le Bradford-cling et le lorest-marble.

Localité : les Clapes; fort rare. (Coll. de II. de Lamberlye.)

Mrtilus texusthiatus, Mu.

Hunster in Goldfuss, Pctref. Germ., t. II, p. 176, pl. 131, fig. : 5.

Localités: Longwy (glacis), Gravelotte (mames), Fontoy, Gorze, (marnes bleues); partout assez commun. 
Hytilus asper, Sow. sp.

Modiale aspera, Sowerby, Min. Conch., p. 2!, pl. 203, Ml. 212, fig. \&-6.

D'Orbiginy (Prodrome t. I, p. 312) classe cette espece dans le Bathonien; Bromn (Iudex paloont.) la range dans le Néocomien, et Sowerby dans le Cornbrash.

Localité : les Clapes; fort rare.

Nytilus pamasiticus, Desl. sp.

Modiola parasitica, Eudes Deslongchamps, Mém. de la Soc. linu. de Norm., t. Vi, 1838. Ném. sur les coquilles fossiles lithophages, p. 228, pl, 9, fig. 4 ly à 46 .

Localité : les Clapes, dans l'Isastrea Babeanu; assezrare.

Mrtilus Incluses, Desi. sp.

Modiola inclusa, Endes Deslongchamps, l. c., p. 226, pl. 9, fig. 39-40.

Localités: les Clapes, dans les Istrstrea babeana et serialis, très-commun; Gravelotte (marnes); fort rare.

Irtiles pelchel, Goild. Goldfuss, Petref. Germ., t. II, p. 177, pl. 131, fig. 8, $a, b$.

Localités: les Clapes, avec les précédentes espèces, Gravelotte (marnes); assez commun.

\section{Mrtilus lonsdaei, Mor. et laye.}

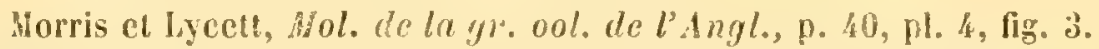

Localités: les Clapes; assez rare. Gravelotte (marnes), Longyy (glacis), Vontoy; asse\% commun.

Mrthus giganteus, Quens!. sp.

Modiola gigantea, Quenstedt, Jura, P. 4.39.

Cette espèce, de très-grande taille, est assez abondante dans les deux zones du Bajocien, où elle se présente assez généralement à l'état de moule, le test étant d'une ténuité el d'une fragilité extrêmes.

Localité : les Clapes; fort rare. (Coll. de H. Colliez.)

Mytilus sowerbyaus, Sow. sp.

Hodiola plicata, Sowerby, Min. Conch., p. 295, pl. 248, fig. 1. Mylitus plicalus, Coldfuss, Petref. Germ., 1. II, n. 17\%, pl. 130, fig. 12 u(non fig. 12 b).

Mytilus Sowerbyanus, d'Orbigny, Prodrome, 1. 1, 1. 282, no 378 , Bajocien et Bathonien.

Cette espèce, très-abondante dans le bajocien inférieur, est au contraire fort rare dans le bathonien.

Localité : Gravelotte (marnes); fort irare. 


$$
\text { Mroules gibmosts, Sow. sp. }
$$

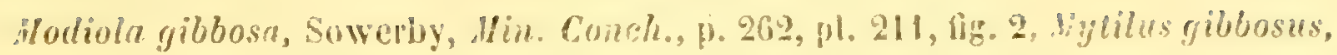
Goldfuss, Petref. Giem. 1. 11, 1. 176, pl. 131, fig. 4.

Colle espice assez rare et tris-pelite dans les marnes blenes de Gravelolle, sc trouve jartout ailleurs avee assez d'abondance et avec uno bien fulus grame lailie.

Localifés: Lonrowy (les gracis el pres du cinctiche), (iorze, lontoy, les clapes, Amaniblr, Ferreviller, Grareloite, ete.

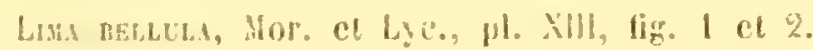

Horris et Lyeett, Hol. de lu gr. ool. de l'Angl., p. 30, pl. 3, fig. 9, non Lima

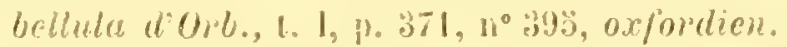

L. Iesta ovato-ubligua, levigata, anfice convexu, abruple trun ta, postice subcompressu, rotunduta, aniculis provis equalibus, lunula magr "exeava! , superficie striis radiantibus tenuibus, nonmnqum obsolctis, strib anticis di:linctis, subdistantibus, posticis crebris counescentibus.

binensions : longrneur :is-11 millim, hanten $89-12$ millim.

Coquille ovale-oblique, renlle dans le haul, tronquéc en abat, compriméc en arrière, arquée diuns le bas; oreilletles pelites el igales; lunnle grande el concave; surface, dans le jenne âge, ornéc le stries régalières trís-serrées et ponetuées; dans l'adulte, surface lisse, quelyues stries obsolètes prés de l'oreilletic postéricure, plis d'aceroissement marqués par de fubles sillons.

Wous arons emprunté litteralement la description latine et nous avons eru devoir feprésenter cette espèce, les auteurs n’en ayant donné que le jeune âge, que nous indiguons par ses dimensions.

Localitis: Gorze (le cometiere), Manbottel (Ialus du chemin de fer), Longwy (calcaire); partout assez rare.

\section{Lime succird, Wh.}

ilunster in Golufuss, Pelref. Germ., \&. II, p. 84, pl. 102, fig. 4.

Cetle espece, trés-commume dans le bajocien inféricur, est caractérisce par les profonds et larges sillons yui sẹparent les cótes.

Localite: liampe de Cons-la-Grandville; fort rare.

\section{Liva Notati, Goldi.}

Goldfuss, Petref. Gemm., 1. II, 1. 83, p1. 102, fig. 1. Horbigny, Prodrome, t. I, p.371, $1^{\circ} 394$, oxfordien.

Localite: Thancourt; assez commun. 
JaMA ovals, Sow. sp.

Plagiostoma ovale, Sowerby, Min. Conch., p. 167, pl. 11\%, fig. 3.

l.ocalilé : Longwy (glacis); assez rare.

Lima IMPREssa, Mor. et Lye.

Morris el Lyeett, Mol. de la gr. ool. de l'Angl., P. 27, ,1. 3, fig. 8.

Ciefle espèce est ornée de côtes ués-plates à peine visibles, rúgulières, légèrement onduleuses, à inlervalles linéares et ponetués.

localité : longry (glacis); assez rare.

Lima Duplicats, Sow. sp.

Plagiostoma duplicatum, Sowerby, Nin. Conch., p. 376, pl. 3.39, โig. 3.

Cette espèce a la même forme et les mèmes ornements que le limea duplicala, elle s'en distingtte pal une taille souvent 3 fois plus grande et par son corselet non costelle et lisse.

Localié : Gravelolte; assez commun.

Une varićć trés-abondanio a ses grosses côtes alrondies, tardis qu'elles sont airues dans l'espece typique; la forme el les dimensions sont du reste identiunes.

LIMA proboscinea, Sow.

Suwerby, Win. Conch., H. 307, p!. 264.

Cefle espice se présente en grande abondance dans les deux couches du bajocien, fort rare dans le fuller's, plus abondante dins le corallien, où elle acquiert une taille gigantesuluc.

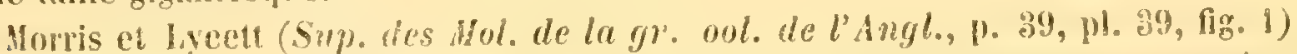
ont reproduit le $L$. pectimiformis, Scht., arec les mêmes caractères que l'espèce ligure par suwerby. Ces auteurs ajoutent que a probablement la coquille élait attachec par lune des valves" (probably was attached by me of the valves to the ground).

Tontes les himes sont munies d'un byssus et le L. proboscidea en particulier en possédait un irés-gros, à en juger par l’ouverture que présentent les valves et leur hord fortenent renrersé.

Incaliés: Gravelotic, Longry (glacis); fort rare.

$$
\text { lima plictivoines, Sow. sp. }
$$

Plagiostoma pectinoides, Sowerby, Hin. Conch., p. 167, p1. 113, fig. 1. Lima pectinoides, Sow. Goldfuss, Petref. Germ., t. II, p. 87, 1). 102, fig. 12.

Dans Sowerby, cette espece ligure ì torl sur la planche sous le nom de P. cardiiformis, le texte rectifiant l'erteur; du reste, la figure est incorrecte at la description incomplete; elles donnés par Goldfuss s'appliguent exactement a nos échantillons. 
Cette espece se distingue par des côtes (13) grosses et aiguës, à larges intervalles; toute la surface est couverte de plis trè-fins concentriques et de stries verticales très-serrées.

Localité: Gravelotte (marnes); assez rare.

Lama textstrata, lib.

Ilunster, in Goldluss, Petref. Germ., 1. 11, P. 82, pl. 101, fig. "̈.

Nous possédons plusieurs variétés qui présentent des modifications dans le nombre et la forme des côtes, qui deviennent d'autant moins nombreuses qu'elles sent phus larges et plus aphaties.

Localité : les Clapes; très-commun.

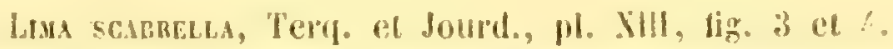

L. lestr parva, ovala, gibbosa, costis clatis, scabris, radimlibus, requalibus, interstitio majoribus ormata.

l)imensions: Iongueur 6 millim.; lıauteur 8 millim.

coquille petite, ovale, gibbeuse, ornée de còtes ćlevées très-écailleuses, rayonnantes, égales, plus larges que les intervalles.

Localite: Corze (cimetière); fort rare.

\section{Lima gmboss, Sow. \\ Sowerly, Min. Conch., p. 206, pl. 152.}

Localites: Gorze, Longwy, Gravelotte, Clapes, Fontoy; partout tris-commun.

LiMEa nUPhicats, Mu.

Nunster in Golufuss, Petref. Germ., t. II, p. 102, pl. 107, fig. 9.

Golduss, qui altribue a Lamarch la création de ce genre, dit qu'il lient it la fois aux Limes par la forme de la coquille et aux Pétoncles par la disposition des dents de la charnière.

Nous ne trouvons pas foujours les dents placées sur les ailes, par conséruent verticales ou obliques; parfois elles sont laterales et alors entièrement transverses, analogues à celles qu'on voit sur certaincs espèces de peigne et mêue sur des ralves flhuitres vivantes on fossiles.

D'Obbing (Prodrome, t. I, p. 283) altribue ex genre à Bromn ef place cetle espece dans le bajocien, qui ne la renferme pas dans nos parages.

Un de nos échantillons oecupe une loge dans un polypier, il l'instar d'une coquille perforante; la coquille, en raison de son habitat, a changé de forme et pris celle d'une Saxicave; clle est biillante en arrière et les crochels sont atrophies.

Localités: les Clapes, Gravelotte, fontoy; partout abondant. 


\section{LIMEA.}

Fous yossédons des coquulles microseofiques qui présentent bien les caractères du genre, et dont les ornements se composent de cótes rayonnantes ariondics: dans les intervalles se trourent des stries concentriques.

Localite: Fontoy, diverses couches; assez rare.

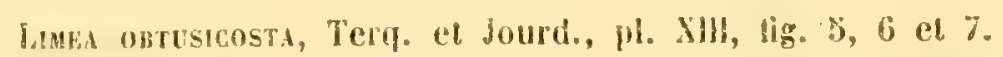

L. Lest minima, suborbiculari, gibbosa, circiter compressa, coslulis regularibus, radiantibus, oblusis, interstitio lineari ornata, umbonibus mediants.

Dimensions : longueur I millim.; hauteur 1,3 millim.

Coquille très-petite, suborbiculaire, renfléc dans le milieu, dépriméce sur te pourtour, ornée de côtes rayonnantes, obtuses, à intervalles linéaires; crochets renflés, inédians, charnière munie de dents sériales verticales, bord interne crénelé.

Localite : Fontoy; fort rare.

Avavel DigrTit, Desl.

Eudes Desiongchamps, Hom. de la Suc. linn. de Norm. Ł. VI H/em. sur

le Prekilopleuron Buckitand, p. 72, pl. 1, lig. 9.

Dimensions : longueur 2:-35 millim.; hateur $19,2-27$ millim.

La premiere série de chiffes done les dimensions de la figure produite par Deslongchamps; la seconde série, celle de l'échartillon que nous possédons, qui, du reste, présente une forme identique é est oncé de 12 côtes munies d'un molongenent spiniforme.

Locatités: les Clapes; font :ure. Gravelotte (manes); abondant.

Aricula echivita, Sow.

Sowerby, Min. Conch., 1. 290, pl. 243 , fig. 1-6.

Localités: dans loutes les localités et partout très-abondant.

Ivicues reculats, Goldf.

Coltiuss, Petref. Germ., 1. 11, p. 132, pl. 121, fig. 6.

Localités: dans tontes les localités el partout très-abondant.

Nous avons eu quelques depites s'il convenait de maintenir celte espèce ou de la réunir à la préećdente, les auteurs quiles ont mentionnées étant peu d'accord sur leurs caractères distinclifs.

Sowerby définit de la sorte l'A. echinata: "Loquille subuvale, gibbeuse, ornéc de nombreuses côtes (16 d'après le dessin), treillisées sur la valve creuse; valve plate, lisse, à oreillette antérieure pointue.

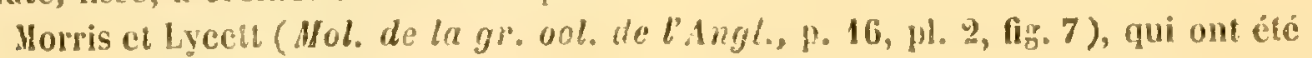


à meme de connaitre les types de Sowerby, donnent cette description poir l'A. "ehinatr sow.: a Coquille ovale-oblique, a oreillettes petites et égales, valve gauche convexe, ornee de nombreuses côtes rayonnantes, alternant avec d'autres plus petites et de lignes iransversales, formant des noeuds avee les grandes eótes; valve droite, subplane, lisse el marquée de lignes rayonnantes obsolètes. „

Goldfuss ('Petref. Germ.), après la deseription de l'A. tegulata, ajoute: * La valve sauche est plus bombée que la droite, qui est de mème que l'autre ornée de lignes, caructère qui sert ì ta ristinguer de l'A. cehinata Sow., espice qui se troure près de Ifet\%.

Dans l'indication des localités, Goldfuss nous apprend qu'il a recu des échantillons de l'.1. tagulate de Voltz et provenant de la grande oolithe des environs de Iel\%.

Les figures parfaitement dessinces se rapportent bien à nos coquilles du bathonien, mais, d'après les caracteres tirés des ornements de la valve droite, on voit que Jorris et lycett sont en désacend avee Goldfuss, quant à la spécification de l'espece de sowerby.

Worbigny (Prodiome, t. 1, P. 283, n" 402) place l'A. tegulata Goldf dans le bajocien et l's. celimata sow. dans te bathonien (p. 313, $\left.n^{0} 311\right)$.

Cette opinion ne résout pas la difficulté, attendu que les deux espieces se fouvent réunies dans une seule et mìne couchs.

Quenstedt (Jura, p. 38\%, pl. 34, fig. :) représente l'A. cchinata sans lignes transversales et la valve droite moitié plus courte que la gauche, tandis que partout nous les tronyons égales en hauteur.

Bronn (Index paloont.) prend ponr l'A. echinute la figure I de Sowerby, e'esta-dire la valse droite, et donne pour synonime l'A tegulata Goldf.; la figure 1 de Sowerby, la valve gauche, devient IA pectiniformis Br.

Dams sa Lethea (édition 1837), Bronn reproduit d'après Quenstedl les caractères de l'A. echinat $\ell$, que nous arons unentionnés plus haut, et donne, dans son atlas, pl. 27, hig. 13, une contuilie à oreillettes éyales el loules deux aiguës, par conséquent une espice qui difite de toutes les autres.

En fésumé, ce fossile se présente aree une abondance extrème depuis les premiers strates du calcaire à polypiers jusłu'aux depóts supéricurs du balhonien, où Il disparait en même temps que l'Oslrea teuminata et l'A. Parkinsoni. Dans toute eette suecession de dépouts, les fossiles possècient une forme qui reste constante, bien que les önements varient légèrement, mais pas suffisamment pour qu'il soit possible d'y reconnaitre plusieurs especes.

Dans les premiers dépòts, la coquille n’a que 16 à 18 côtes sensiblement égales; dans les couches qui suecedent elle en a de 20 à 30 , de grosses côtes alternant aree d'autres plus petites; enfin dans les couches a A. Parkinsoni, les fossiles

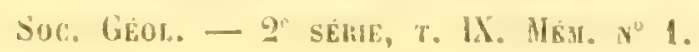


ont de 40 à 46 côtes; les lignes transversales déterminent sur les côtes des nœuds, restes de gouttières qui se terminaient en épines allongées.

La valve droite est généfalement ornée de 10 à 12 côtes rayonnantes obsolétes; l'oreillette postérieure est très-courte, aiguë dans la valve droite, obtuse dans la sauche,

Goldfuss ayant bien décrit ce fossilt: et l'ayant exactement représenté, nous admettrons la détermination ąu'il a établie, à l'exclusion de celle des autres, quelle que soit leur date d'antériorité.

Aviculi.

Nous avons trouvé dans plusieurs couches de Fontoy des Avicules dont la valve gauche se rapporte à l'A . tegulata, dont les deux ailes sont plus ou moins égales, et dont la valve droite est parfois lisse. Ces coquilles étant toutes de très-petite taille, nous n'avons pu y reconuaitre des caractères suffisants pour en faire une ou plusieurs espèces, et nous nous contenterons de les mentionner.

Localié: Fontoy; assez commun.

Avicula costata, Sow.

Sowerby, Min. Conch., p. 290, pl. 244, fig. 1.

Cette espèce se distingue de l'A. digitata Desl. par ses intervalles ornés de nombreuses côtes, 8-10, qui semblent appartenir à la partie ẹnidermique et disparaissent facilement.

Localité : Longwy (glaeis); fort rare.

Iricula transversa, Terq. el Jourd., pl. XIII, tig. 8.

A. testa transversa, clongala, gibbosa, costis radiantibus (12) remotis, regularibus, in spina elongata desinentibus, in medio aliguot minoribus ornata, umbone obliquo, neuminato, auriculis brevibus, requalibus.

Dimensions : longueur 35 millim.; hauteur 25 millim.

Coquille allongée, transverse, renflée, ornée de côtes rayonnantes, élevées, espacées, régulières, et de quelques-unes plus fines dans le milieu de la coquille, se terminant toutes en pointe allongée; crochet oblique, renflé, acuminé, oreillettes petites, égales.

Cette espèce, dont nous ne possédons que la valve gauche, se rapproche de l'A. digitata Desl. et s'en éloigne par sa forme transverse.

L'échantillon est pétrilié par la silice, circonstance fort rare, que nous n'avons rencontrée qque dans une térébratule ( $T$. bullata, le centre est géodique et entouré d'un cercle de calcédoine), et d'autant plus remarquable, que la roche ne renferme pas de nodules de silice.

Loealité : Longwy (glacis); fort rare. 
Avicuis котавиis, lerg. et Jourd., pl. Xlil, fig. 9, 10 et $\mathrm{H}$.

A. lesta convexa, oblique ovala, juvenile et adulle ad umbonem costulis clatis, radiantibus idque lineis concentricis clallratis ornata, in medio lavigat et nitidn, postice uliquot costis obsoletis instructa, umbone prominulo, auricula anticu brevi, obtusa, postica acuminata.

Dimensions : Jeune âge.

Longueur. 3 millim.

Adulte.

Itauteur. . . . . . . . 3 millim.

13,j-12 millim.

$13-13,3$ millim. compriméc sur son pourtour, obliquement ovale, dans le jeme âge et dans l'adulte près' du 'crochet, onée de côtes rayonmantes élevées el de lignes conentriques, formant un élégant treillis, lisse e brillante dans te milieu, marquée postéricurement de quelques côtes peu saillantes, à peine visibles; crochets renflés, saillants, oreillettes inégales, la postéricure triangulatre acuminée, l'antéricurc très-courte.

Par l'entière conservation des ornements du jeune âge, cette coquille semble ètre formée par la superposition de deux enquilles d'espèces différentes.

Localité : Longwy (glacis); assez rare.

Aveula ixonata, Terq. et Jourd., pl. Xlll, fig. 12.

A. testa ovata, valva sinistra convexa, obliqua, leviguta idgue nitida, valva dextra concava, lavigata, arriculis inoequalibus, posticis acuminatis, anticis oblusis.

Dimensions : longueur 18 millim.; hauteur 23 millim.

Coquille, valve gauche convexe, obliquement ovale, lisse et mème brillante, valve droite, concave et lisse; oreillettes inégales, les postérieures triangulaires et aiguës, les ontéricures obtuses.

Celle espece, par sa valve droite concave ct par l'absence de tout ornement, se distingue de toutes les autres, bien que par sa forme elle se rapproche de l'A. tegulata.

Localité : Conllans; foit rare.

Genvilua spatulata, T'er!. cl Jourd., pl. Xill, fig. 15.

G. testa elongata, angusta, incquivalvi, antice altenuata, gibbosulu, postiec spalulata, rmbone in valva sinistra mominulo, areuato, subacuto, ala antica acuminata, postica elongata, valva dextra compressa, umbone vix perspieuo; cardine fovealis 4 instructo.

Dimensions : longueur 80 millim.; largeur 20 millim.

Coquille allongée, étroile, inéquivalve, très-rétrécie et un peu renflée en avant, comprimée el spatuléc en arrière, ornée de stries concentriyues, rérulieres; sur la valve ganche, crochet allongé, arrué et aigu, aile antérieure acumince, aile postéricure plus longrue que ia moitié de la coquille; sur la valve gauche, erochet 
à peine visible; charniere munie de \& fossettes ligamentaires et do: 2 ot 3 dents allongées.

Localités: Longwy (glacis), Montigny-sur-Cher's, Nouillon-Pont (Meuse); assez rare.

Gervilum laxceolata, Mu.

Nunster iu Goldfuss, Petref. Germ., t. II, p. 123, pl. 113, 1ig. 9. Gervilla acula, Phillips, Géol. du York, pl. 9, fig. 36, non Gervillia acula, Sowerby, Hin. Conch., p. 325, pl. 510, fig. 6 .

Cette espece est plus grêle et plus courte que la figure domée par Goldfuss, bien gu'elle coneorde avec la deseription.

Localités: Gravelotte (marnes), Fontoy; assez rare, surtout entière.

$$
\begin{aligned}
& \text { Genvelis acut1, Sow. } \\
& \text { Sowerly, Win. Conch., p. 325, pl. } 310, \text { fig.6. }
\end{aligned}
$$

Norris et Lycett, Hol. de la gr.ool. de l'Angl., p. 20, pl. 3, fig. 12.

Les denniers auteurs considèrent comme synonimes de celte espèce les $G$. lanceolata Golkf. et le G. siliqua Desl., opinion que nous ne potvons admettre, attendu que nous possédons toutes ees especes et que nous leur arons reconnu des différences spécifiques bien justifićes.

Localite : Fontoy (marnes); assez rare.

Gervilla subcyundrica, Hor. et bje.

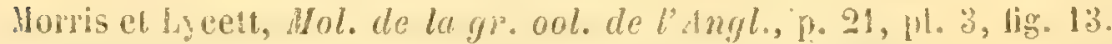

Cette espèce, étroite, allongée, digitiforme, se distingue frar le crochet très-aigu de la valye gauche, et par son aile postéricure très-allongée, occupant les 2 ? de la longueur de la coquille; nons la possédons également du bajocien supérieur (calcaire à polypiers).

Localité : langrnevaux; asse\% commun.

Germun rerixoms, Dest.

Eudes Jeslongchamps, Mem. de la Soc. lim. du Calvados, 1824, Hém. sur les lierv., p. 126, pl. 1, 2 et 3.

Deslongchamps fait observer que cette espece se trouve assez souvent avee son test transformé en calcaire spathique (les Clapes); parfois elle perd ce test, quoiqu'il soit très-épais, cl ne présente plus que la partie épidermique trèsceailleuse (Fontoy) marnes.

1 ans nos contrées, cette espèce atteint la mème taille ( $1: 3$ à 20 cent. de longueur sur $4-5$ de largeur) que dans la Normandie.

Cette espèce se distingue du G. aviculoides, Sow. par une forme bentoup moins grèle et par son aile antérieure beancouy moins aiguę. 
D'Orbigny confond ce fossile aree l'aviculoüles qui est parfaitentent représentée pai Goldfuss (pl. 1:3, fig. 8), et que nous possétons le l'oxfordien des raches noires.

Localites: les clanes, fontoy; très-abondam, mesque tonjonts on fragments. (Con!, de It. Colliez, une valve droite complète.)

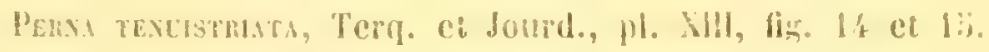

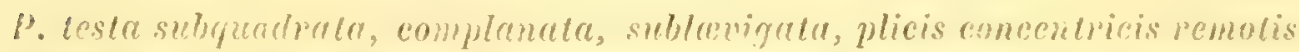
striisque temibus, reguinibus, absoletis aruake.

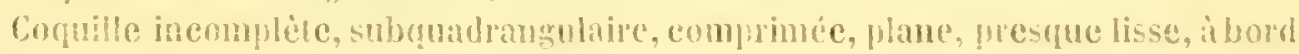

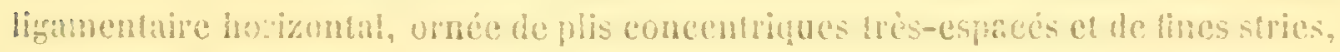
régulicires, obsolites.

lien que mous ne possédions quine eoguille incomplète, nons arons dir en fare une espece ent se distingue de tontes les autes par su surfue lisse et brillante, fout en présentant des orments dine giande finwe ef isibles senttement it la loupe.

tencalice : les Clapes; lont rare.

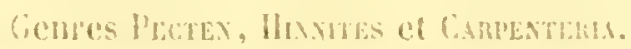

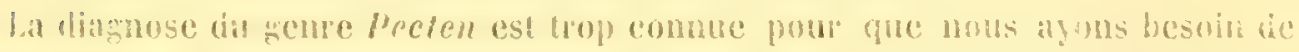
lat reproduire; elle ne présente d'aillew's aucun doute pour la classifieation des

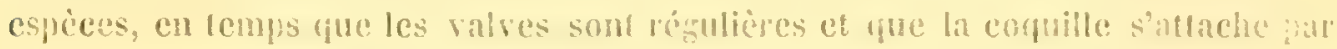
le mojen diun byssus (1).

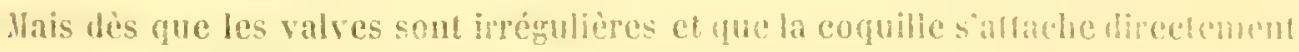
a un support, la classification des espèces a beancoup varié.

Defrance a établi le genre llimiles prour des peirnes drformés, attachés par une partic de la surface de la valre droite; la valve ganche est libre, plate el prote. ainsi tine l'autre des cotes myonnantes nombreuses, sensiblement igales, les iniepralles oceupés par des cotes plus petites; ornements qui se rapprochent de cenx des Spondyles.

Goldfuss (Petrep. Germ.) prenant ces ornenents pour base de classification et ne cherchant pas à conuatre les caractères de la charniere, a rangé ces fossiles parmi les Spondyles.

Deslongehamps (Hém. de la Soc. linn. de Norm., 1. XI) a élabli le genre Carpenteriu, pour des coquilles qui représentent exactement les caracteres des Hinnites, et qui s'attachent soit par le crochet, qui devient une large surface

(1) l'our distinguer les deux balves entre elles, nous plagons les l'eignes dans la meme position glue les autres actpha'es, c'est-à-dice les crochets en dessus el louverture pour le passare du byssus ea arant; it en resulte une valre droite et une valve gauche dont la désignation reste constante, quelle que soil la forme de la roquille, inéquivalre on equivalfe; dans le premier ras, la ralre bombápe pst la droile cl la valve plate est la gauche. 
carrée, soit par unc partie plus ou moins grande de la valve droite. Comme pour les llimiles, la valve gauche est libre, plate, ou plus ou moins bombée. Les coquilles sont parfois ornées de grosses côtes et ressemblent assez à des Huitres, les oreillettes n'étant pas détachées par un sillon.

On remarque, dans le lias ainsi que dans l'oolithe, des coquilles qui, possédant les ornements et la forme des Itimites, s'attachent, contrairement à ccux-ci, par la valve gauche et ont la valve droite libre. Hans ce nombre, se trouve le Spondylus tubreulosus cie Goldfuss, qui fait observer (p. 94) "que ce fossile a parfois sur, le milieu de la valre deux eôtes tuberculenses phus élevées que les autres. „

Morris et Lyeett se sont servis de ce caractère, quelque léger qu’il fủt, el ont rapporté la variété indiquée par Goldfuss au Pecten gradus, Bean,-sous le nom de Hinnites grudus, el en ont représenté une valve droite.

Quensted (Ju'a) a reproduit les deux valves de cette espèce et reconmaissant dans la valve gauche adhérente le caractère propre aux Peignes, des oreillettes très-développées et parfailement détachées, en a fait ie P'ecten luberculosus.

Il résulte de ce court exposé qu’il se trouve deux sortes de fossiles: dans l'une, les coquilles sont attachées par la valve droite, ont des orcillettes indistinctes sur les deux valves et se rapprochent ainsi d'un Spondyle qui serait privé de dents à la charnière; dans l'autre, les coquilies s'attachent par la valve gauche, et malgré cette aftache, ectte valve montre des oreillettes distinctes, identiques à celles des Peignes, les ornements se produisant analogues à ceux des Spondyles.

Nous ne croyons pas que les caractères tirés de l'adhérence de l'une des valres et de leurs ornements soient suffisants pour classer ces fossiles dans les différents genres que nous avons mentionnés; mais si l'ou réunit toutes ces coquilles irrégulières dans le genre limnites, il convient de faire observer que ce genre, à l'instar de quelques Ciastéropodes et Bivalves contiendra des Cossiles dextres et senestres, e'est-à-dire que les uns seront adhérents par la valve droile, et les autres par la valve gauche.

A la difficulté de classement que nous venons d'exposer, il faut encore en ajouter une antre : nous possédons d'une part, du lias moyen, une coquille équivalve et éfuilatérale, à-valves plates, ornées de côtes rayonnantes grandes et petites, à oreillettes indistinctes; d'une autre part, de l'oolithe inférieure, une coquille très-inéquilatérale et inéquivalve, ornée de côtes concentriques, dont la valve droite a la forme d'une Lime et la valve gauche celle d'un Pcigne; ni l'une ni l'autre de ces deux coquilles ne montre de points d'attache, et cependant la première, quoique régulière dans sa forme, se rapprocherail des llinnites par ses ornements, comme la seconde, malgré son irrégularité, se rapporterait aux Peignes par ses côtes concentriques et par les oreillettes de la valve gauche.

Par ces motifs, nous classerons tous ces fossiles farmi les Peignes. 
Pecter fibrosus? Sow.

Sowerby, Min. Conch., p. 185, pl. 136, lig 2.

Goldfuss, Petref. Germ., 1. 11, 1\%.46, pl. 90, fig. 16.

L'espèce indiquée par ces auteurs se rapporte exactement aux fossiles si abondants dans l'Oxfordien; ceux du bathonien en difièrent par dé côtes plus nombrenses et moins saillantes.

Localité : les Clapes; fort rare.

Pecter henucostates, Hor. el Lye.

Horris et Lyeett, Mal. do la gr. ool. de l'Angl., p. 10, pl. I, lig. 16.

Cente espèce a ses valves inćgalement ornées: la valve gauche a des côtes rayonnantes dont la saille devient plus prononcée près du bold inférieur; la valve droite n'est ornée que de fines côtes coneentiques.

Nos échantillons diffèrent de l'espèce figurée par un plus grand nombice de cótes rayonnantes.

localité : les Clapes; fort rare.

Pecter anticclates, Schl.

Pectinites articulatus, Schlothein, Petref., p. 227 et 228. Goldiuss, Pelref: Garm., t. II, p. 4.7, pl.90, fig. 10.

Cette espèce se trouve en grande abondance dans le bajocien supéricur (le calcaire á polypiers) et présente la mème taille el les mêmes caractères que dans le bathonien.

Localite: : les Clapes; assez commum.

Pecten leNs, Sow.

Sowerby, Min. Conch., p. 253, pl. 205, lig. 2-3.

Celte espèce se trouve abondamment dans les deux zones du bajocien comme dans celles du bathonien.

Localités: Longwy (glacis), les Clapes, Gravelotte (marnes); partout commun.

Pectevecingulatus, Phil.

Phillips, Géol. du York, pl. b, lig. 11. Goldfuss, Petref. Germ.,

ᄂ. II, P. 74, pl. 99, lig. 3.

Goldfuss indique eette espèce pour le lias ainsi que pour l'oolithe du Wurtemberg.

Notre échantillon est plus orbiculaire que celui représenté par Goldfuss, se rapproche davantage, quant à la forme, du P. Wollastomensis, Nor. et Lyc. (Mol. de la gr.ool. de l'Angl., p. 33, pl.33, fig. 2), et en diflère par ses ornements : de fines côtes concentriques qui ne sont pas squarreuses dans le bas.

Localité : Ies Clajes; fort rare. 


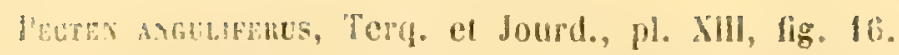

() lesta suborbiculari, aquivalui, subconvexa, costis oblusis, recta el sinistra value costis radiuntibus (cirea 43 ) in medio acule angulatis ornata, auriculis mugnis inequatibus, tenuisime striatis.

Dimensions : longueur 11 millin.; haten 11 millim.

Corulle amsci large que hatle, éunivalre, suborbiculaire, légèrement convexe; sur lia valre gatuche, ornce de côtes obluses rayonnantes à droite el à gauche, soulées a angle aigu dans le milieu, intervalles presque linéaires; sur la valve

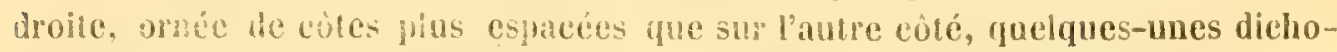
tomes près du bord inféricur, intervalles couverts de stries concentrinnes.

Localités: Longwy (glacis), Gerze (cineliere); fort rare.

Pectes eximith, Terq. el Jourd., ph. Xlll, ligg. 17.

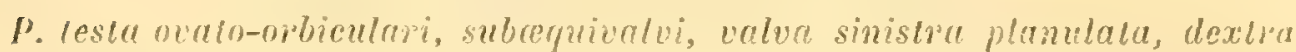
subonvexa, juenile costulis, nodulosis, radinntibus, areualis, adulte costulis

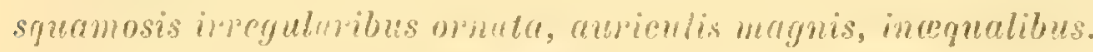

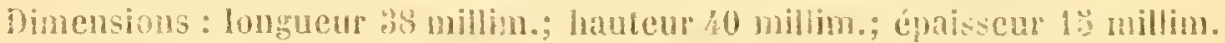

Coųuille ovale-orbiculaire, subéquivalre, subé(juilatérale, comprinéc sur la valve gauche, subconvese sur la droite, oméc dans le jeune àge de côtes fines, noduleuses, rayonmantes, régntières ef arquées; dans l'adulle de côles fines, aplaties, écailicuses, imégulières; meillettes grambs, incigales, ornées d'un réseau de eroisillons obliques.

Cette espice a la forme du P. lens et en difterc par ses ornements.

Sur denx échantiltons frès-bien conservés, nous arons trouvé les sillons tracés par un Talpine.

bucalite : frarclote; alssez rare.

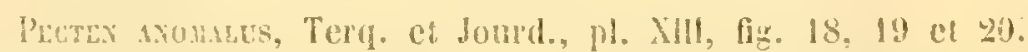

P. lesin suborbiculari, aquivalvi, conrexo plana, valva dextra castis elatis, squamosis, sinistra costulis paliuntibus rigeminatis, utrinque duabus, deinde solis, strisquc concentricis, strictis ormatu, auriculis incisis, incequalbus, concentrice striatis, anlico acuminuto.

Dimensions : longucur 19,5-1' millim.; hauleur 21-16.

Coquille suborbiculaire, équivalve, plano-convexe, valve droite ornée de 10 côtes élevées, écailleuses, valve gauche ornée de côtes rayornantes, réunies par trois dans le milieu, par deux de chaque côté, puis isolées, et de stries transversales, trèi-fines, serrées, néguliures; orcilleltes fortement inciscées, inégales, ornées de stries concenthiques, l'antérieure trangulaire et aiguë.

La valve gauche mésente quelzues varations dans la disposition des eòtes; les trois séries médianes sont parfois accompagnées de quelques fines côtes; parfois toutes les séries ne comprennent que deux cotes. 
Cette espece souvent confondue avee le $P$. vaga is Sow. de l'oxfordien, dont elle a les grosses cútes de la valve droite, s'en distingue par un plus grand nombre de còtes el une forme plus allongée.

Localités: entre Conflans et Vriauville, Gorze (cimetière); assez commun.

Pectear tuberculosus, Goldf. sp,

Spondylus tuberculosus, Goldfuss, Petref. Germ., t. II, p. 93, pl. 105̈, fig. 2. Pecton gradus, Bean, Mag. Nat. Hist. Kinnites yradus, Bean, sp. Morris et Lycett, Sup. Mol. de la gr. ool. de l'Angl., p. 33, pl. 33, fig. 10. Hinnites tuberculosus, d'orbigny, Prodrome, t. I, p. 283̈, n 427, Bajocien. Pecten tuberculosus, Quenstedt, Jura, p. 434, pl. 39, fig. 9 et 10.

Cette espèce se montre constamment attachée par la valve gauche; elle est abondante ainsi que sa variété dans la zone supéricure du bajocien.

Localité: Gravelotte (marnes); asse commun.

Pecter nusudenexsis, Lyc.

Horris et Lycett, Sup. Mol. de la gr.ool. de l'Angl., p. 33, pl. :3, f. 4 a, b et $c$. Localité : Gorze, Gravelotte (marnes); assez rare.

Pecten spatulatus, linem.

Pecten spatulatus, Romer, Ool. Geb., p. 26, pl. 18, fig. 22.

Quenstedt, Jura, p. 433, pl. 59, fig. 13.

Localité : Gravelutte (marnes), Longwy; très-commun.

Pegtex annulitus, Sow.

Pecton anmulatus, Sowerby, Min conch., p. 539, pl. 342, fig. 1; non Pecten anmulatue, Sow. Goldfuss, Petref. Germ., t. II, p. 49, pl. 9I, fig. 2.

Cette esprèce est presque équilatérale, et les deux valves sont également bombées.

Localité : Gravelotte (marnes); assez commun.

Pecten Germania, Goldf., sp.

Non Pecten annulatus, Sowerby, L. C. Pecten anmulatus, Sow., Goldfuss. I. C. Pecten Gormania, d'Orbigny, I'rodrome, t. I, p. 314, no 332.

Cette espèce est inéçuivalve et se distingue ainsi de la précédente, les ornement reslant à peu près les mèmes.

Localité : Gravelotte (marnes); fort rare.

Soc. GŔol. - ge SERE, T. IX. - Hès. No 1. 
Pecten echinatus, Goldf. sp.

Spondylus velatus, Goldfuss, Petref. Germ., t. II, p. 94, pl. 10\%, tig. 4, Oolithe supérieure.

Hinnites velatus, d'Orbigny, Prodrome, t. I, p. $374, \mathrm{n}^{0} 455$, oxfordien

Notre échantillon se rapporte exactement à la description et à la figure que Goldfuss donne pour une valve droite.

La coquille est légèrement bombée et ornée de côtes rayonnantes, arrondies, régulières, alternativement grosses et étroites, couvertes de petits nouds déterminés par les stries concentriques.

Cette espèce adhérente par la valve gauche, dinère du pecten tuberculosus, qui a 6 ou 7 petites côtes dans les intervalles qui séparent les grossses côtes.

Goldfuss ayant déjà établi un Pecten velatus pour le Nuschelkalk, nous avons dủ changer la dénomination de l'espèce oolithique.

Localité : Longwy (les glacis); fort rare.

Pecten semispinatus, Terg. el Jourd, pl. Xlli, fig. 21 el 22.

P. testa suborbiculari, convexn, costis (circa 20) cequalibus, conformibus, radiantibus, supra obtusis, levigatis, infra aculis, spinatis, plicisque transversalibus ornata; a uriculis levigatis, antica parvula, postiea magna, margine dentato.

Dimensions : Iongueur 21 millim.; hauteur 21 millim.

Coquille suborbiculaire, convexe, ornéc de côtes (environ 20) égales entre elles et aux intervalles, dans le haut, lisses et obtuses, dans le bas, aiguës et épineuses; munie de stries concentriques à peine visibles dans le haul, très-marquées dans le bas; oreillettes lisses, l'antéricure bencoup plus petite que la postérieure; bord denticulé.

Cette espèce, dont nous regrettons de ne posséder qu'une seule valve et même inconplète, est remarquable par ses ornements, qui semblent être le résultat de l'accolement de deux coquilles appartenant à deux espèces, tant la partie supérieure diffère de l'inférieure.

Localité : Longwy (glacis); fort rare.

Pectem limeformis, Terq. et Jourd., pl. XIV, fig. 1, 2 et 3.

p. testa maxime inaquilaterali et inaquivalvi, costulis tenuissimis, concentricis ornata, valva dextra convex", obliqu, limeformi, auriculis parvis, obscuris, sinistra paramper concava, obliqua, auricula antica prelonga, incisa, postica brevi, area denticulata.

Dimensions : longueur 50 millim.; hauteur s2 millim.; épaisseur 12 millim.

Coquille très-inéquilatérale et inéquivalve, ornée de côtes très-fines, concentriques et espacées; valve droite convexe, oblique, en forme de Lime, à oreillettes courtes, peu distinctes; valve gauche légèrement concave, oblique, à aile anté- 
rieure grande, séparéc par un sillon, la postérieure courte, corselet orné d'unc série de dents aiguès.

Cette espèce se rapproche dı $P$. annulatus par ses ornements et s'en distingue par sa forme irrégulière; la valve gauche ne montre aucune trace d'attache et les ornements qui se sont bien conservés témoignent que la corfuille n'élait pas adhérente.

Localité : Gravelotte (marnes); fort rare.

\section{Pectin.}

Nous avons trouvé dans une alvéole de perforant, un peigne lisse, suborbiculaire, très-comprimé, dont les oreillettes sont atrophiées et dont les valves ont pris une disposition obliqque, très-inéquilatérale.

Localité : les Clapes; dans un Synastrea; fort rare.

Plicatula ristulosa, Hor. el Lye.

Norris et Lycett, Mol. de la gr.ool. de l'Angl., p. 15, pl. 2, fig.

Cette espèce se distingue par ses plis rayonnants simples quoiøue irréguliers et munis d'épines fistuleuses; elle se frouve avec les mèmes caractères dans to bajocien supéricur (le calcaire à polypiers).

II. Colliez a trouvé un magnifique échantillon à Wohécourt (Meuse) dans la zone à A. quereinus avec la grande Tereb. lagenalis et l'Ostrea Wiltomensis.

Localité : Gravelotte (marnes); fort rare.

$$
\begin{aligned}
& \text { Ostrea saxdalixa, Goldf. } \\
& \text { Goldfuss, retref. Germ., t. II, p. 21, pl. 79, tig. } 9 .
\end{aligned}
$$

Cetle espëce ne possède aucun pli, s'attache par toute sa surface inféricure et a son bord relevé sul tout son pourtour; elle se présente avee abondance dans la zone inférieure du bajocien, devient plus rare dans la zone supérieure, pour se retrouver abondamment dans le bathonien et mème dans l'oxfordien.

Localités: Ies Clapes, Amanviller, Longivy (glacis); très-commun.

Ostrea obscura, Sow.

Sowerby, Min. Conch., p. 503, pl. 488, fig. 2 à 4.

Cette espèce, qui s'attache par la partie antérieure du talon, présente tous les caractères et les varichés de forme de l'O. irregularis du grès de Hettange.

Localités: les Clapes, Amanviller, Lonğwy (ğlacis); très-commun.

Ostrea gregarea, Solw.

Ostrea gregarea, Sowerby, Hin. Conch., p. 163, pl. 111, fig. 1-2.

Ostrea gregarea, Sow. Ostrea rastellaris, Ostrea nodosa, Goldfuss, Petref.

Germ., t. 11, p. 7 et 8, pl. 7i, fig. 2-4.

D'Orbigny (Prodrome, t. I, p. 37k) réunit toutes ees espèces en une seule qu'il place dans l'oxfordien. 
Nous possédons un très-grand nombre d'échantillons rẻpondant aux diverses figures données par Goldfuss, et nous trouvons, d'une part, que les trois espèces peuvent être réunies en une seule, les modificalions résultant de l'àge, du mode d'attache et de la nature du support; d'une autre part, nous devons reconnaitre que nous ne pouvons établir de différence entre les fossiles si abondants du bajocien supérieur et du bathonien avec ceux de l'oxfordien; nous voyons ceux-ci toujours pétrifiés par la silice, tandis que les autres sont toujours calcareux.

Localités : les Clapes, Gravclotte, Longwy (glacis); géneralement assez rare.

Ostrea crenata, Goldf.

Goldruss, petref. Germ., t. II, p. 6, pl. 72, fig. 13.

Ostrea subcrenala, d'Orbigny, Prodrome, t. I, p. $28 \%$.

Nous ignorons par quels motifs d'orbigny a modific la détermination de Goldfuss; il a considéré cette espèce comme identique à l'o. Marshii de Phillips, et a placé l'O. Marshii Sowerby, dans le callovien et l'oxfordien.

Dạns nos parages, l'O. Marshii de Sowerby et de Goldfuss se trouve plus particulièrement dans le bajocien supérieur (le calcaire à polypiers), et l'O. crenato dans le bajocien inféricur (le calcaire ferrugineux).

L'échantillon de la zone à $A$. Parkinsoni se rapporte bien à cette dernière espèce : l'huitre était attachéc par la majeure partie de sa surface; ses côtes sont simples, peu saillantes, tandis qu'elles sont fourchues et très-aiguës dans 1'O. Unarsii.

Localité : les Clapes; fort rare.

Ostrea sulcifens, Phil.

Phillips, Géol. du York, p. 123, pl. 9, fiğ. 33̀.

D'Orbigny, Prodrome, t. 1, p. 283, rend celle epece synonime de l'O. exarala, Goldf., et ajoute en observation : a Ces deux noms ne valent vien, ils sont tirés du corps sur lequel l'huitre était fixée : la prenière sur un corps allongé, la seconde sur une Ammonite. »

Nous avons ces deux espèces sous les yeux et nous voyons qu'elles diffèrent complétement: l'O. sulcifera est allongée, a le talon très-long, linité de chaque côté par un large sillon, le pourtour est sinueux; le test est si épais que la valve supérieure ne saurait reproduire la forme du support, la valve inférieure ne s'attachant que par l'extrémité.

L'O. exarata a le test très-mince, papyracé et le talon très-court, sans sillon latéral; la valve supérieure reproduit la forme du support, la valie inféricure étant complétement soudée.

Localité : les Clapes; assez commun. 
Ostrea Marshi, Sow.

Sowerby, Jin. Conch., 1. 103, pl. 48, fig. 1-3.

Cette espèce, très-abondante dans le bajocien supérieur, devient d'une rareté extrême dans le bathonien.

Localites: Longwy (marues), Gravelolle (marnes); fort rale.

$$
\text { Ostrea conccitrici, Mu. }
$$

Golufuss, Petref: Germ., 1. 1t, 1. 3, pl. 72, fig. 9.

Cetle cspece à test tres-inince ant once de ptualques fines cotes rayonnantes, obsoletes; bien que la valve soil altachéc par toute sa surface inférieure, le pourtontr est relcve de unclues millimeites.

Localités: les Clapes; assez rare. Au pied de la rampe des fiénivara; assez. commun. Ranguevaux, à la suiface des bloes; très-commun.

$$
\text { Ostrea teberosa, Mu. }
$$

Inumster in Goldfuss, Pelref: Germ., Ł. II, p. 5, pl. 72, lig. 1.

Cefte espice est attachéc yar toute sa surface sur des morecaux de iignile.

Localités: Longwy (glacis), Thiaucourt; fort rare.

(Ostraci Kinmu, Ziet.

Vieten, Verst. Wurt. p. 60, pl. $43, \mathbf{1 i g} .2$.

Cetle espèce se distingue trés-facilement de l'O. costalu, Sow.: elle est déprimée, généralement projetéc en arière et ornée de nombreuses et fines còtes; tandis que l'O. costata est bombéc, aussi large que haute et ornée de grosses côtes aiguës.

D'Orbigny, Prodrome, 1.1, p. 237, n $^{0}$ 260, a commis une double crueur en placant cette espèce lans lo Toarcion et ch l'indiquant aux Génivaux, près de Metz, où il ne saurait y avoir trace de marnes liasiques.

Localités: Cutry, Joppécourt, se contime dans la Jieuse a Wohécourt et Spincourt (collection de H. Colliez); partout très-abondant.

\section{Ostrci Gibntici, Mart.}

J. Hartin, Notes sur quelyues espèces peu comnes du Ballonien, Vémoires de l'A cud. de Dijon. 1. X, 1862, p. 12, pl. 3’, fig. 16-24.

Cette cspèce est petite, sa taille ne dépassant pas un centimètré, élevée, ornée de grosses còtes dichotomes sur les còtés et munic d'une large attache frontale.

Kous considérons les $O$. Knorrib et Gibriaci comme des variétés de l'O. costata ct appartenant a la meme forme typique; cependant nous maintenons ces espèces en raison de la spécification des horizons qui en ressort: 1० l' $O$. Gibrinci se trouve dans la zone inféricure et dans les premières couches a 
A. Parkinsoni; $2^{\circ}$ l'O. Knorrii est commune dans la zone à A. Prrkinsoni, el se . continue jus(que dans la zone $A$. Quereinus; $3^{\circ} \mathrm{I}^{\prime} O$.costata commence au-dessus de cette dernière zone et se trouve en abondance dans les premiers strates de l'oxfordien.

Localités : zone à A. niortensis et à A. Parkinsoni. Montigny, Longwy, Gravelotte; généralement asse\% rare.

Ostrea aurirobis, Hor. et Lye. sp.

Exogyra auriformis, Morris et Lycett, Mol. de la gr. ool. de l'Angl.,

$$
\text { p. 3, pl. 1, fig. } 7 \text {. }
$$

Il conviendrait peut-être de conserver la dénomination première pour marquer le moment où la forme Exogyre apparait pour la première fois; unique d'abord dans le bathonien, elle devient très-abondante dans l'oxfordien et se continue jusque dans les terrains crélacés.

Localités : Conflans; fort rare.

$$
\text { Ostrea wiltoxensts, Lye., pl. 14, fig. } 4 \text {. }
$$

Morris et Lycell, Suppl. des Mol. de la gr. ool. de l'Angl., p. 108, pl. 34, fig. 1. Forest-marble.

Cette espèce est trigone et ressemble beaucoup à l'o. deltoidea du kimérigdien.

Localités : Conflans, Jarny, ainsi qu’à Wohécourt (Meuse) (collection de M. Colliez); partout très-abondant.

Ostrea acumixata, Sow.

Sowerby, Mirt. Conch., p. 184, pl. 133, fig. ë, non lig. 4.

Cette espèce, comme ses congénères, varic beancoup dans sa taille et dans sa forme : trois fois aussi longue que large près de Hayange et d'Angeviller, elle est aussi large que haute dans les autres localités.

Localités: Gravelotte (calcaire et marne), Gorze, Auboué, Fontoy, Amanviller, Jaumont, les Clapes, Longwy (g! acis); partout très-abondant.

Ostrea Sowerby, Sow. sp.

Ostrea acuminata, Sowerby, Min. Conch., p. 184, pl. 135, fig. 4. Ostrea Sowerbyi, Morris et Lycett, Mol. de la gr. ool. de l'Angl., p. 4, pl. 1, fig. 3.

Ces auteurs ont établi deux espèces pour des fossiles que Sowerby avait réunis sous une seule dénomination; l'O. acuminata (fog. 3), abondant dans le bathonien inférieur et moyen, disparaît en même temps que l'd. Parkinsoni dans la zone supéricure, et est remplacé par une coquille à forme trigone, douéc de plis trèsespacés à intervalles lisses et concaves.

Nous sommes disposés à croire que l'O. acuminata et l'O. Sowerbyi ne constituent qu'une seule et même espèce, et que la forme trigone n'est qu'une modi- 
ficationde la forme allongée; en effet, parmi les pelits fossiles, notis en trowrous tous les passages.

Cependant, comme les fossiles douts le es formes se montrent avee une abondance extrème dans des zones trés-difrérentes, nous maintiend'ons les leux espèees, mais uniquement pour servir de guide dans la délimitalion des horizons.

Localités : Conflans, Jarny, ainsi qu’à Wohécourt (Meuse) (collection de M. Colliez); très-abondant.

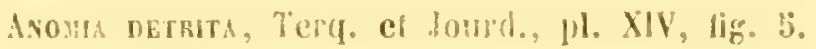

A. testu ineyutariter orbiculnri, gibbosula, concentrice plicren, costulis radiantibus conferlis ornuta.

Dimensions : largeur 33 millim, hantent 30 millim.

Coquille irrégulierenent orbiculaire, légèrement renllée, orrée de gros plis d'accroissement et de fines côtes rayonnantes et serrées, crochct non marginal.

Nous avons douné plutôt les earactères du genre que ceux de l'espèce, et il est, en effet, très-difficile de distinguer entre elles toutes les eoquilles qu . nous possédons les différents terrains et assises.

Depuis le lias inféricur jusqu'au bathonien inclusivement, nous avous de ces coquilles papyracées et ideutiquement ornées de stries rayonnantes (de 6 a 40 mill.); pour la plupar nous possédons la valve inférieure avec son ouverture caractéristique; de la sorte nous ne saurions les confondre avee le gemre Pluchnopsis lor. et Lyc., don la valve inférieure n'est pas percée.

En signalant cefte espèce dans le bathonien, nous n'arons voulu que compléter la série malacologique.

Localités: les Clapes, parasite sur direrses coquilles, entre autres le Trigonia costuta; assez rare.

Aroma nonderera, Terg. et Jourd., pl. XIV, ligo 6 et 7.

A. Lesh purvela, orbiculari, costulis moniliferis mediantibus ormata.

Dimension : diamètre 6 inillim.

Coquille orbiculaire, valve supéricure bombée ct ornée de séries rayonnantes de jetiles perles; valve inféricure plane, lisse et muic d'une large ouverture; surface interne nacrée.

Localité : lontoy, $10^{e}$ couche de marne; assez dare.

\section{BHACHOPODES.}

LIEMTIIYRIS costata, D'Orb.

Dorbigny, loodrome, 1. 1, p. 286, no 4.48, bajocien.

Localité : les Clapes; assez rare. Conflans, Jarny; très-commun.

Lingue Beanh, Phil.

Phillips, Géol. du York, p. 128, pl. 11, fig. 24.

Localités: Hainbottel, sur le talus de la route, Longwy, dans le calcaire sousjacent aux marnes des glacis; très-commun. 
Rirncioneled acuticosta, Helm.

Terebratula acuticosta, Helmann, Zieten, Verst. Wu*t., p. 58, pl. 43, 6. 2.

Localité : les Clapes; très-commun.

Ruynchonela variass, Schl. sp.

Terebratula varians, Schlotheim, Petref. Kunde, 1, p. 967.

Localité : les Clapes ; très-conmun.

Rirrchosella Theodori, Schl. sp.

Schlotheim, Petref. Kunde, 63.

Cette espèce se distingue lu $R$. couticostn par ses gros plis (6 à 8) et une forme plus arrondie.

Localités: les Clapes et sur les glacis des forts qui entourent Longwy; trèsabondant.

RuYichosella corchisa, Sow. Sp.

Terebratula concinna, Sow., Min. Conch., p. 128, pl. 83, fig. 8.

Localités: Thumeréville, Jarny; très-commun.

Rirychonela gladriplicata, Ziet. sp.

Terebratula quadriplicata, Zieten, Verst. Wurt., p. 35, pl. 41, fig. 3. W'Orbigny,

Prodrome, t. 1, p. 286, $\mathrm{n}^{\circ} 438$ et p. $315, \mathbf{n}^{\circ} 343$.

Localités : Conflans, Jarny; fort rare.

RHYxchosela coxcinvoldes, D'Orb.

D'Orbigny, Prodrome, t. 1, p. 315, n"3:46.

Localités: Wohécourt, Conflans, Jarny; assez commun.

Terebratula subresurisita, d'Orb.

D'Orbigny, Prodrome, t. I, p. 287, $\mathrm{n}^{0} 454$, bajocien, non Terebralula resupinata, Sowerby, Min. Conch., p. 204, pl. 150, fig. 3-4.

Localités : les Clapes, Gravelotte; fort rarc.

Temebratula emargivata, Sow.

Sowerby, Min. Conch., \%. 451, pl. 435, fig. 9-12.

Localités: les Clapes, Iongwy, Gravelotte, Fontoy, Conllans, Gorze; partout ires-commun.

\section{TERebratula onnthocephala, Ziet.}

Lieten, Petref. Kunde, 1, 284.

Localités : les Clapes; fort rare. Conflans, Friauville, Longwy; très-abondant.

Terebratula Maxillata, Sow.

Sowerby, Min. Conch., [). 432, 1)1. 436, fig. 7-9.

Localités: Gravelotte, Fontoy, Gorze, Longwy; partout très-abondant. 
TEReHatula Lagesalis, Mu.

Munster, Verzeich. der Verstein. Bairculh, 1836.

Quenstedt, Jura, p. 492, pl. 66, fig. 9-12.

DOrbigny, Prodrome, t. 1, p. 377, $\mathrm{n}^{\cap} 473$, oxfordien.

Nous possédons dans notre département des coquilles qui présentent deux deux formes très-distinctes: l'une, relativemeni petite, a de 35 à 38 millim. de longneur; est carrée à sa partic frontale et caractérise la couche inférieure du bajocien; l'autre, beaucoup plus allongée, a de 50 à 60 millim. de longueur, est plus ou moins rétrécie à sa partie frontale et ne se trouve que dans la zone supé rieure, dans les parties limitrophes des départements de la Moselle et de te Heuse; la zone moyenne ne nous a pas encore donné de fossiles de l'une ou de l'autre forme.

Davidson considèce la grande coquille comme représentant le type de l'espèce, les autres n'en constituant que des variétés, qui l'accompagnent habituellement; il signale leur présence, pour l'oolithe de l'Angleterre, depuis le fuller'searth jusque dans le cornbrash, en passant par la grande oolithe et le bradford-clay.

Tout en admettant l'opinion de Davidson, nous nous voyons cependant obligés, en raison de la station de ces fossiles, d'y établir une division ou au moins une variété pour la petite espèce.

Localités: plateau de Chenières, Conflans, Jarny, Thumeréville, Wohécourt; partout assez abondant.

Temebratula lagenalis, Nu. Var. minor.

Localité : cimetière de Longwy; assez rare.

Terebratula bullata, Sow.

Sowerby, Min. Conch., p. 431, pl. 433, lig. 6-8.

Localités : Thumeréville, Conllans, Jarny; très-commun. Longwy; très-lare.

Thecioed tibrosula, Terq. et Jourd., pl. XIV, fig. 8 et $\%$

7. testa ovata, lavigata, umbone producto, apice acuto, triangulari, valua superiore antice gibbosula.

Dimensions : largeur 2 millim.; hateur 1,3 millim.

Coquille ovale, lisse, un peu plus large que hate, libre au bord postérieur, a crochet allongé, triangulaire, aigu au sommet, valve supérieure régulièrement ovale, gibbeuse en avant.

Nous ne connaissons pas la disposition interieure des valves, mais la forme de la coquille diffère complétement de l'esnèce qui se trouve avec tant d'abondance dans le bajocien supérieur.

Localités : (iravelotte, sur l'Ostrea M/arshii, Longwy (glacis); fort rare.

SOC. GEOL. - 2e SERIR, T. IX. MEY. N 1 . 
Crania costellata, Terq. et Jourd., pl. XIV, fig. 21 el 22.

C. valva superiore orbiculari, irregulariter convexa, hemisphorica, vertice subcentrali, costulis irregularibus, radiuntibus arnata, cicatricibus magnis, subaqualibus, anticis ovalibus, posticis rotundatis, dissepimenti ramis arcuatis, angustis, timbo costula lavigata circumdato.

Dimensions : longueur 11 millim.; largeur 9 millim.; hauteur 3 millim.

Coquille, valve supérieure, orbiculaire, irrégulièrement convexe, hémisphérique, à sommet subcentral, ornẻe de fines côtes irrégulières et rayonnantes; impressions musculaires grandes et subégales, les deux antérieures ovales, les postérieures rondes; bras des cloisons arqués, sous forme de petite côte arrondie, bord interne garni d'une côte étroite et lisse.

Notre échantillon présente le cas exceptionnel où deux individus se sont intimement soudés; extérieurement la coquille est irrégulière, plus large en avant qu'en arrière; à l'intéricur on remarque 8 impressions museulaires el à peine une légère trace de soudure.

Localités : les Clapes, Fontoy (espec̀ce microscopirque); fort rare.

\section{ANNELIDES.}

Galeolaria socialis, Bromn.

Broun, Lethaa, p: 472, pl. 27, tig. 9.

Serpulu socialis, Goldfuss, Petref. Germ., 1. I, p. 233 , pl. 69, fig. 12.

Cette espèce se trouve abondamment dans les deux zones du bajocien et se continue jusque dans l'oxfordien.

Localités : les Clapes, Gravelotte, Fontoy; partout assez commun.

Ditrypa fontinexsis, Telog. el Jourd.; pl. XIV, fig. 19, et 20.

D. Lesta minima, conica, recta, rugosa, plus minusve strangulata, antice ad aperturam attenuata.

Dimensions : longueur '̉ à 8 millim.; largeur 1 à 1,30 millim.

Coquille très-petite, conique, droite, rugueuse, plus on moins munie d'étranments, rétrécie près de l'ouverture.

Bronn (Index palcont.) rectifie avec raison la dénomination de Ditrupa en celle de Ditrypa, Berckeley, rectification que nous avons adoptée.

Localité : Fontoy; très-commun.

Serirta FlACCIDA, Hu.

Ifunster in Goldfuss, Petref. Germ., t. 1, p. 234, pl. 69, fig. 7.

Localités: les Clapes, Fontoy, Gravelotte, Longwy; commun. 
Serpula thtragoxa, Sow.

Sowerby, Min. Conch., p. 622, pl, 599, tig. 1-2.

Localités: les Clapes, Fontoy, Gravelotte, Longwy; assez commun.

Serpula euadrlatera, Goldf.

Goldfuss, Petref. Germ., t. I, p. 230, pl. 68, fig. 9.

localité : les Clapes; assez commun.

Serpula scobraula, Terip. et Jourd., pl. XIV, fig. 10, 11 et 12.

S. testa abbreviata, spirata, uno visibili anfractu, circiter rotundata, supra depressa, squamis dissectis scobiniformibus oblecta, apertura orbiculuri.

Dimensions : hauteur $\$$ millim.; diamètre 21 millim.

Corpulle courte, enroulée, formée d'un tour de spire visible, arrondie sur les côtés, déprimée en dessus, couverte d'écailles déchiquetées sur le bord et donnant à la surface l'aspect d'une lime, ouverture arrondie.

Localité: Ies Clapes; fort rare.

Serpula velitebrilis, Sow.

Sowerby, Min. Conch., p. 623, pl. 599, tig. 3.

Loealités : les Clapes, fontoy, Gravelotte, Longwy; commun dans les premieres Incalités. rare dans la dernière.

Semplla tricainata, Sow.

Sowerby, Min. Conch., p. 633, pl.608, tig。 当 el 4.

Goldfuss, Petref. Germo, t. 1, p. 290, pl. 68, fig. 1i.

localité : les Clapes; très-commun.

Senpled pextigond, Goldt.

Goldfuss, Pelref. Germ., 1. 1, 1. 230, pl. 68, 1ig. 7.

Localité : les Clapes; très-commun.

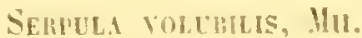

Hunster in Coldfuss, Petref. Germ., 1. 1, 1. 233, pl. 69, ti \%. 2.

Morris et Lycent (Mol. de la gr.ool. de l'Angl., p. 120, pl. 14, f. 8) rapportent à cette espèce le Vermicularin nodus, Phil. (Phillips, Géol. du York, 1. 1, 1. 124, pl. 9, (ig. 24), ct le rangent dans les Amélieles.

Bromn (Index pulcoont., p. 1362) classe au contraire cette espece parmi les Vermets et la rend synonime des $\boldsymbol{S}$. convoluta et lituiformis.

D'après cette maniere de voir, qu'il convient de elasser parmi les Vermets toutes les Serpules enroulées en spire irrégulière, ou attachées seulement dans le jeune âge, il faudrait encore mentionner les S. trocheatu, - macrocephala, - 
grandis et heliciformis; mais alors il faudrait agir contrairement à ce qui se produit dans les mers actuelles.

Localités : les Clapes, Longwy (glacis), Conflans; assez rare.

Senpula plicatilis, Mu.

Munster in Goldfuss, Petref. Germ., 1. 1, p. 229, pl. 68, fig. 2.

Loealités : Conflans, Jarny; commun.

Serpula limax, Goldf.

Goldfuss, Petref. Germ., t. I, p. 227, pl. 67, fig. 12.

Localités : Longwy, Clapes, Friauville, Conflans, Vohécourt; commun.

Serpula conforats, Goldf.

Goldfuss, Petref. Germ., t. 1, p. 228, pl. 67, fig. 13.

Localités: Longwy, Gravelotte, Jarny, Gorze, Conflans; commun.

Serpula gordialis, Goldi.

Goldfuss, Petref. Germ., t. 1, p. 231, pl. 69, fig. 8.

Localités : Longwy, Clapes, Fontoy; commun.

\section{CRUSTACÉS DÉCAPODES.}

\section{PALINURES.}

Glyphea crassa, Opp.

Oppel, Mémoires de la Soc. des Sciences nat. du Wurt., 1. XVII. Mémoires sur les crustacés jurassiques, p. 109.

Localité : Longwy (glacis), Friauville; lort rare.

\section{BRACHIURES.}

Nous avons trouvé dans les couches inférieures des marnes à A. Parkinsoni de Fontoy, quelques débris (pinces) presque microscopiques qui appartiennent à cette division, sans qu'il soit possible d'indiquer le genre auquel il conviendrait de les rapporter.

Localité : Fontoy; fort rare.

\section{CRUSTACÉS OSTRACODES (1).}

Les fossiles de cette famille sont généralement abondants dans les marnes, qui

(1) Si le temps et les circonstances le permettent, nous les publierons dans ce travail, pour le rendre aussi complet que possible; en cas contraire, nous les mettrnns à fa suite de nos publications sur les foramioifères. 
ont, dès leur dépót, subi un tassement suffisant pour empêcher le passage des courants acidules; abondants à Fontoy, ils sont fort rares ou ont même complétement disparu dans la majeure partie des localités que nous avons explorées.

Accompagnant toujours'les forminiferes, ils ont cu, comme ceux-ci, à passer par les mêmes phases d'abondance of de destruction.

\section{BRYOZOAIRES.}

Tehebripora (1) steldfem, Terq. et Jourd. PI. XIY, fig. 16.

$T$. sulcis rectis, in stellis raliantibus dispositis munita.

Testier formé de sillons très-étroits, rectilignes, se coupant sous différents angles et déterminant des étoiles ì 8 ou 10 rayons.

Localité : Longwy (glacis), sur le Lime bellula; fort rare.

Terebripora ramiciformis, Terq. et Jourd. PI. XIV, fic 18.

T. sulcis radiciformibus, angustissimis, rectis vel plicatis, munila.

Testier formé de sillons très-étroits, disposés en forme de radie ..es, droiter ou genouillées, ne formant pas de mailles.

Localités: Gorze (cimetière), sur le Lima bellula; fort rare.

Terebripoba latesulcata, Terq. el Jourd. PI. XIV, fig. 17.

T. sulcis late impressis, imegulariter texturatis, quadiangularibus vol polygonalibus munita.

Testier formé de larges sillons, disposés en un réseau irrégulier, à mailites larges, carrées ou polygonales, visibles à l'wil nu.

Localité : Gravelotte (marnes), sur le Lime tenuistriata; assez rare.

Terebripora meroglyphica, Terq. et Jourd. Pl. XIV, fig. 15.

T. sulcis angustissimis, in lineis dentiformibus disposilis munita.

Testier formé de sillons très-étroits, disposés en lignes brisées et en forme de dents, ne déterminant pas de réseau.

Localité: Gravelotte, sur le Pecten exaratus; assez commun.

Hetenopora conifera, lior.

Mnrris, Cal. des foss. de l'Angl., p. 39. Jules IIaime, Mém. de la Soc. yéol. de France, 2o série, t. V. Desc. des Bryoz. foss. de la form. jurass., p. 208, pl. 11, fig. 1.

Localités : Longwy, Romain.; assez commun.

Heteropora pustulosa, J. Ilaime.

Jules Haime, $L . C .$, p. 210, pl. 11, fig. 2.

Cette espèce très-abondante dans les deux zones du bajocien, se présente de

(1) Nous admeltons le mode de classification exposé lans l'étude sur les Bryosoaires perforants de M. Fischer, bien que, malgré loules nos recherches, nous n'ayons pu parvenir à observer la forme 
même dans le bathonien inférieur et daus la grande oolithe (Langrune).

Localités : les Clapes, Longwy (glacis), Hainbottel; commun.

Proboscisa bucu, J. Haime.

J. Haime, Bryozoaires fossiles de la form. jurass., p. 168, pl. 6, fig. 10 a el b.

Localité: Longwy, sur le Plicrtula fistulosa; fort rare.

Diastopora Micheusi, d. Haime.

J. Haime, Bryozoaires fossiles de la form. jurass., p. 188, pl. 8 , fig. 8 a à $d$.

Localité : Amanviller; fort rare.

Dustopond Wrathi, J. llaime.

Jules Haime, L. C. p. 186, pl. 8, fig. 气.

Localité : fiorze (cimelic̀re); fort rare.

Dhastopora scobixula, J. Haime.

Jules llaime, $L . C$. . ). 186, pl. 8, fig. 6.

Localité : fontoy; fort rare.

Stontropon Terocen, J. Ilaime.

Jules Haime, $L$. C. p. 164, pl. 6, fig. 金.

Localité : les Clapes, sur un Hytilus; fort rare.

Stomatohora minotonoldes, J. Haime.

Jules llaime, L. C. p. 163, pl, 6, fig. 2.

Localités: entre larny. et Conflans, sur une Ihû̉re, Longwy, sur Tepebralulr, Thiaucourt, sur une Lime; assez rare.

Berearea Arcinda, J. Ilaime.

Jules Haime, L. C. p. 180, pl. 9, fig. 11.

Localite: Longwy (glacis) sur une Terebratule; assez rare.

Brexice diluvisi, Lam.

Lamouroux, Exp. méth. des gemes de polyp., p. 81, pl. 80, lig. 3ł. Jules Haine, L. C. i. 177, pl. 7 , tig. 2.

Localités: Longwy (glacis), sur des Térébratules, des Huitres, des Trigonies; frès-commun.

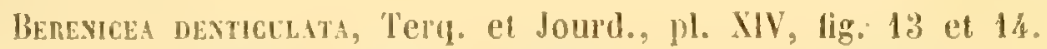

Testier épais, gibbeux, formé de plusieurs couches superposées; testules distincts sur toute Ieur longueur, se terminant en un tube rertical et denticulé sur le bord, 8-10 dents arrondies, persistantes, inégalement espacées, très-serrées près du bord.

Cette espèce diffère du $\boldsymbol{B}$. diluvianu par ses testules relevés; du $\boldsymbol{B}$. Archiaci par l'absence de plis transverses; mais, comme celui-ci, elle tend à s'aceroître en épaisseur, et forme des mamelons de plusieurs millimètres de hauteur.

Localité : les Clapes; assez rare. 


\section{ECIHNODERMES.}

\section{Collymites mixgers, Des Moul.}

Des Noulins, Et. sur les Ee., p. 369, n 13. Cotteau, Paléont. frang. Ech., p. 41, pl. 6 et 7, hig. 1 à b. Disaster ringens, Agassiz, Ech. de la Suisse, t. 1, p. b, pl. 1 , fig. 7 i 11 .

Localités: Gorze (cimetière), les Clapes; rare.

$$
\text { Prgeres Mromelisi, Col. }
$$

Cotleau, Él. sur les Ech. lle l'Yonne, 1. I, p. 70, pl. 3, fig. 7. Cotteau,

Puléont. frone. Ech., 1). 209, pl. 37.

l.ocalités: darny, Gorme (cincticre); assez rare.

$$
\text { Clupecs Plati, Klein. }
$$

illein, Nut. disposit. Ech., p. 22, pl. 12.

Cotteau, Paléont. frane., [1. 191, pl. 31 et š2.

Clypeus putella, Igassiz. Eeh. de la Suisse, t. 1, p. 36, pl. 8, 1. . 1 à 6.

Cefte espèce fort rare dans la zone inférieure, foisonne dans toutcs les localités de la zone moyenne, qu'elle ne dépasse pas.

Localités : Longwy, les Clapes, Gravelotte, Jarny, Thiaucourt, Fontoy, ele.

$$
\text { Clypeus Iluall, Ag. }
$$

Agassiz, Ech. de la Suisse, 1. 1, 11. 37, pl. 10, ligr. 2 à 4.

Cotteau, Palcunt. franr., p. $216, \mathrm{pl} .59$.

Localités: les Clapes, Graveloite, Gorze (cincticre); assez commun.

$$
\text { Chyeus Bolizay, Mich. }
$$

Michelin, in coll. Cotteau et Triger, Ech. du départ. de la Sarthe, p. 6. pl. I1, fig. \& et 5. Colleau, Paléont. franc., 1). 199, pl. 55 et $3 \%$, fig. 1 et 2.

Localités: Conflans, rive droite de l'lron; fort rare.

Licinomassus clunicularis. BI. Sp.

Nucleolites clunicularis, BI. Blainville, Dict. LX, p. 188.

Localités: les Clapes, Gravelotte; commun.

$$
\begin{aligned}
& \text { Holectypus depressus, Ah. } \\
& \text { Agassiz. Mon. des Gal., pl. 10, fig 8-9. }
\end{aligned}
$$

Localités: Gravelotte, les Glapes, Longwy, Wohécourt; commun.

$$
\begin{aligned}
& \text { P'edna arayulosa, Ag. } \\
& \text { Agassiz, Cat. syst., p. } 67 .
\end{aligned}
$$

Localité : descente de Montigny-sur-Chiers; fort rare. 
Pebind gigas, Ag.

Agassiz, Cat. syst., p. 24 .

Lacalite : Burons de Longwy, près de la Sauvage; fort rare entier:

Agrosalevia spinosa, Ag.

tgassiz. Ech. de la Suisse; p. 39, pl. 18, lig. 1-\$.

D'Orbigny, Prodrome, p. 320, Bathonien.

Localité : Gorze, et près de Puxicux; assez rare.

Acrosalenia complayata; Ag.

Agassiz, Cat. syst., p. 46. d'Orbigny, Prodrome, p. 290, Bajocien.

Localité : Gorze (cimetiere); fort rare.

Diamema complakatum, Ag.

Agassiz, lichin. de la Suisse, p. 16, pl. 17, fig. 31-33. D'Orbigny,

Prodrome, p. 346, Callovien.

Localité : lirayclolte; asser. commus.

Astemacanthos bolthicun, Terq.

Notis avons provisoirement classé dans le genre Asteracanthion un Stelléride attaché par sa surface inférieure et muni de quatre bras étalés, le cinquième renverse et indistinct; ce fossile se trouve mentionné dans le bulletin de la Société d'Histoire naturelle de Metz, 18\%, p. B4, où il est bien représenté, mais incomplétement décrit.

Dimensions : longueur d'un bras 48 millim.; largeur à la base 31 millim.; diamètre du centre 40 millim.

Bras formé en dessus de 10 rangées régulières de pièces (30) plus ou moins triangulaires on pentagonales, embriquées, grandes et distinctes; en dessous, d'une grande quantité de très-petites pièces rondes ou polygonales; centre occupé par des pièces plus grandes que celles des bras, mais identiques de forme; côté latéral droit muni d'un disque rond, coralloïde.

L'un des bras, écrasé et renversé à son extrémité, montre une série des lames parallèles de la face inférieure.

Localité : Ranguevaux, près de Hayange, zone du calcaire de Jaumont; fort rare.

ISTEROPECTEX.

Pièces très-variées dans leurs formes et dans leurs ornements, se rapportant évidemment à plusieurs espèces et mème à plusieurs genres.

Localités: Fontoy, Longwy, les Clapes, Gravelotte (calcaire de Jaumont); assez abondant dans la première localité, fort rare dans les autres.

La recherche des foraminifires nous a mis à même de recueillir de nombreux 
débris de rayonnés, qui pour être bien classés et exactement déterminés, demanderaient une expérience et des moyens de comparaison que nous ne possédons pas. Nous nous contenterons d'indiquer les quelques genres auxquels nous les rapportons, et nous mettrons tous ees matériaux a la disposition de celui de nos collègues qui vondrait en faire une étude spéciale.

Astrophytox. PI. XV, fig. 12, 15 et 14, arossi 20 fois.

Spicules en forme d'hamecon, blanes, calcaires, aigus, munis d'une pointe recourbéc à une extrémité el d'un anneau à l'autre, analogues aux spicules d'une espèce virante.

Il est à présumer que parmi les nombreux entroques que renferment les marnes et qui, en sénéral, sont considérés comme des articulations de Pentacrine, il s'en trouve qui, en réalité, appartiennent à des bras d'Astropliton, Asteraconthion, ele.

La formation liasique renferme des spicules analogues.

Localité : liontoy; assez abondant dans toutes les couches.

Ophunella, Ophotrix, Ophocom, Ommer..

Pièces internes et externes des bras ainsi que du disque.

Certaines couches, principalement les plus basses, sont littéralement pétries de ces débris.

Localité : Fontoy; abondant dins toutes les conches.

\section{RAYONES. \\ l'extacrinus.}

Près de Villey-Saint-Etienne (2 kilom. de Toul), M. Dargnics (1) a observé une carrière où un massif de calcarre possède des bancs subordonnés qui se lèvent en dalles el sont couverts de Crinoüdes.

La pétrification a saisi ces animaux vivants sur place, les a conservés dans leur entier et les présente par leur face inféricure. Les Crinoüdes sont très-nombreux, juxtaposés el couvrent de leurs bias.étalés toute la surface des dalles; Ieur reproduction était virace, attendu que plusieurs générations se sont sucéclées pour une très-faible épaisseur de roche, et qu'en fendant ces dalles, on retrouve plusieurs nouvelles séries de fossiles.

M. Dargnies a fait don à notre Musée de plusicurs de ces dalles (l'une a près d'un mètre de longueur) et nous a mis à même de faire une étude complète des Crinoïdes qui les recourrent.

D'une part, comme nous venons de le dire, la suceession des générations étant très-rapplochée, pour une très-faible hauteur; d'une autre part, les articles de la tige étant d'une rareté extrême (sur b̆ grandes dalles, nous n'en arons trouré

(1) M. Dargnies, qui a fait une étule très-suivie du système oolithique de la Mosclle, a prèté un ulile concours pour la description géolorique de ce département, principalement dans sa partic de jonclion arec celui de la Heuse, dans la direction de Gravelolle à Liverdun el de là à Toul.

SOC. GEOL - L SERE, T, IX, MEY. $N^{\circ} 1$. 
qu'une seule série de 3 articles), nous en concluons que ces Crinoïdes étaient, les uns, sessiles ou attachés par une tige très-courte, et les autres libres.

Pentacrinus Dargniesi, Terq. el Jourd., pl. XV, fig. 1 à 7.

\section{La Tige.}

A. La tige est nue et formée de ${ }^{\circ}$ on 6 articles pentagonaux, à angles très-saillants et arrondis; chaque article porte deux tubercules accompagnés d'une dépression à droite et à gauche; la face supérieure nous est inconnue.

$B$. La base de Ia tige est munie de nombreuses bractées droites, très-légèrement coniques, simples, longues, lisses, comprimées; les articles sont ovales, à bord arrondi, avec un léger rétrécissement de chaque côté, qui à sa partie supérieure se prolonge en une pointe conique.

$C$. Le sommet de la tige (que celle-ci existe ou ne soit que rudimentaire) est garni d'un verticile de bractées courtes, coniques, composées d'articles arrondis et lisses, comme dans les Comatules.

2. Le Calice.

Le calice est formé de $\$$ pièces lisses, en éperon en arrière et carrées en avant.

3. Les Bras.

$A$. Les pièces radiales sont au nombre de 2 pour chacune des 3 divisions et sont munies dans le milieu de deux protubérances spiniformes.

$\boldsymbol{B}$. Les pièces brachiales, au nombre de 9 , sont ornées d'un seul tubcrcule, placé alternativement à droite et à gauche, selon la position de l'article; la dernière pièce pentangulaire est munie de deux tubercules; cette disposition se maintient dans toutes les divisions qui suceèdent.

C. Les divisions dichotomiques qui interviennent sont bien plus nombreuses (9 ou 10) sur les bras extérieurs que sur les intérieurs (2 ou 3); les bras se terminent par des divisions d'une extrème ténuité, en forme de chevelure, souvent colorée en brún ou cn pourpre.

Les articles, aussi loin qu'on peut les suivre sur les rameaux à l'aide d'une loupe, se montrent ornés de la protubérance spiniforme, qui reste très-distincte ; la petitesse des articles et leur rapprochement et surtout l'alternance des protubérances à droite et à gauche, font croire que les bras sont armés d'une double langée d'épines.

Dimensions : tige: longueur 0,14 mill.; largeur 0,04 mill.

-.. bractées inférieures : Iongucur 0,80 ; largeur 0,03 .

- bractées supérienres : longueur 0,28 ; largeur 0,02 .

- bras : longueur 0,90 à 0,110 ; largeur des pièces radiales 0,04 .

Toutes les fois que, dans une formation, il s'est produit une agglomération de Crinoïdes, on a donné à la roche le nom de calcaire à entroques; de là son application à des horizons très-différents, alors que cette dénomination ne devait servir que comme renseignement local. 
Ainsi nous comnaissons dans les assises supéricures du calcaire à gryphées arquées, des bancs tellement pétris de débris de Pentacrines, qu'ils pourraient être désignés sous le nom de calcaire à entroques, tout aussi bien que ceux du système oolithique.

Dans la Bourgogne, un calcaire à entroques est l'équivalent de notre calcaire à polypiers et appartient par conséquent au bajocien supérieur.

Dans le Jura, ces calcaires se reproduisent fréquemment ef appartiennent à divers horizons.

Nous ehereherons done à spécifier la position stratigrafique de Villey-SaintEtienne par les principaux fossiles que renferme la roche: A. Parkinsoni, Pholadomya texturata, Ilomomya Vezclayi, Limopsis oolilhicus, Limea obtusicostu, Terebratule maxillata, Clypeus Ploti.

En conséquence le massif de calcaire est placé dans la partic moyenue du bathonien, et répond au calcaire supérieur de Gravelotte; il est recouvert par les marnes à Pecten anomalus de l'horizon de Conflans et par dessus se trouvent les marnes à grande Terebratula lagenalis de Thumeréville. (H. Dargnies.)

\section{EXPLICATION DES FIGURES.}

1. L.es cinq bras, grandeur naturelle.

2. Un bras, avec piece basale, grossi 3 fois.

3. Bras avec radioles, grossi 6 fois.

1. a. b. Tige grossie 3 fois.
(5. a. b. Bractée supérieure, grossie 3 fois.

6. Bractées inférieures, grandeur naturelle. 7. a. b. Bractée inférieure, grossie 3 fois.

Pextachines velgakis, Schl.

Schlotheim, Petref. Wurt., t. 1, p. 327, pl. 1, fig. 6.

Pentacrinus scalaris, Goldfuss. Petref. Germ., t. I, p. 173, pl. 32, fig. 3.

On trouve de nombreuses articulations de la tige et des bras, qui présentent la mème forme et les mèmes ornements que celles que renferme le lias à diverses hanteurs.

Localités: les Clapes, Fontoy, Gravelotte, Longwy (glacis), etc.; partout assez colmmun.

\section{Eugeniacrinus nutays? Goldf.}

Goldfuss, Petref. Germ., 1. I, p. 164, pl. 50, fig. 4.

Localite: Gravelotte (marnes); fort rare.

\section{ZOORIITES.}

Nous avous recueilli à Fontoy des spicules calcaires ou siliceux de plusieurs formes et que nous avons classés suivant leur analogic avec les spicules que contiennent les espèces vivantes. 
Gorfosil.

Spicules variées qui présentent des rapports de forme avec plusicurs espèces vivantes.

Cimrodota. PI. XV, fig. 8 à 11 , grossi 60 fois.

Spicules hémisphériques, creax d'un côté el ornés sur la partie convexe de §o ou 6 lames plates, rayonnant du centre, qui est légèrement déprimé; très-voisins, des spicules du Chirodota violacea sinon identiques.

Conrad Schwager (1) a trouvé ces corps microseopiques dans les marnes oxfordiennes du Wurtemberg el les a considérés comme appartenant à des Ilolothuries; nous possédons des spicules d'llolothuric vivant, et nous devons dire qu'ils ne ressemblent nullement aux spicules fossiles.

Le lias moyen de l'Indre contient également de ces spicules, mais qui diffèrent légèrement de ceux de l'oolithe et appartiennent probablement à une autre espèce.

Awabacia orbultes, Lam. sp.

Fungic orbulites, Lamouroux, Exp. méth. des polyp., p. 86, pl. 83, fig. 1-3.

Anabacia orbulites, d'Orbigny, prodrome, t. I, p. 321, no 434, bathonien;

Anabacia bajociana, d'Orbigny, L. C., p. 292, $n^{\circ}$ 522; bajocien.

Milne-Edwards et Jules Ilaime, dans leur étude sur les polypiers (Pol. de la gr. ool. de l'Angl., p. 120, pl. 25, fig. 3), ont réuni les deux espèces de d'Orbigny en une seule.

Localités: Gorze (cimetière), Auboué sur les hauteurs, Conflans, Jarny; abondant dans toutes ces localités.

Montliyaulia Delabecini, Edy et Haime.

Milne-Edwards et Jules Haime, Hém. de la Soc. géol. de Londres, 18:1. Pol. de l'élag. inf. de l'ool., p. 132, pl. 26, fig. "s.

Localité : Thumeréville; assez commun.

NontLigulta trocholdes, Edw. el Haime.

Milne-Edwards el Jules Haime, L. C., p. 129, pl. 26, fig. 2.

Localité : entre Jarny et Conflans; assez commun.

Isastrea tenuistriata, N'Coy. sp.

Astrea tenuistriata, M'Coy, Ann. and Mlag. of Nat. IItst., S. 2, I. II, P. 400.

Isastrea tenuistriata, Milne-Edwards et Jules Haime. Mém. de la Soc. géol. de

Londres 183̈1, Polyp. de l'ool. inf., p. 138, pl. 30, fig. 1.

Localité : les Clapes; très-commun.

(1) Beitrage zur Kenntniss der mikroscopischen Fauna jurassicher Schichleu, Conrad Schwager, in Muschen, $1860^{\circ}$. 
Isastrea Cosybeari, Edw. el llaimc.

Jilne-Edwards et Jules Haime, L. C., p. 113, pl. 22, fig. \&.

Localité: Ies Clanes; très-commun.

\section{Is.sTREA LIMTATA, Lam. sp.}

Astrea limitata, Lamouroux, Iconog. des Zooph. de Michelin, p. 229, pl. 94, f. 10. Isastrea limilata, Milne-Edwarus et Jules llaime, L. C., p. 114, pl. 23, fig. 2.

Localité : les Clapes; très-commun.

Isastrea seraals, Rdw. et Haime.

Milne-Ldwards et Jules llaime. L. C., p. 116, pl. 24, fig. 2.

Localité: Ies Clapes; très-commun.

Thampastrea Defrancina, Edw. et Haime.

Nilnc-Ldwards et Jules Haine, L. C., p. 139, pl. 29, fig. 3.

Localité : les Clapes; très-commun.

\section{FORAMNIFERES.}

La localité de Fontoy nous ayant donné une quantité exubérante de foraminifères, nous avons dù en faire non-seulement unc publication à part, mais encore ćlablir des monographies pour quelques genres, vu leur extrême abondance et les nombreuses rariélés que nous ayons rencontrées. Nous renyerrons donc pour celte famille aux mémoires que nous avons publiés: le premier contenant huit planches et publié par la Société d'histoire naturelle de Hetz (1867), renferme la monographie du genre dlarginulina, aree 32 espèces; le second, avec 11 planches, et publié par l'Académic de lietz (1869), contient la monographie des cristellaires arec 2/ espéces; un troisième mémoire égalenent publié par l'Académie de Netz (1870), contient les genres Frondicularia, Flabellina, IIaplophragmium, Lingulina, Glanduline, Comuspira, Layena, Nodosaria, Dentalina et Weubina, constituant dans leur cnsemble 72 espèces, comprises dans 8 planches.

Les autres genres, relatirement aussi riches en espèces rque les précédents, sont encore proportionnellement plus abondants que ce que l'on observe en général dans d'autres terrains. Nous nous oceupons de leur étude, et si leur ciassement peut être terminé pour l'époque de la publicalion de ce mémoire, nous les comprendrous dans la nomenclature générale de la faune. Par le nombre des genres, l'abondance des especes et la multiplicité pour ainsi dire infinie des rariétés, l'ensemble de ectle étude donne des movens faciles pour recomaitre des horizons similaires (1); nous regrettons d'être obligés de laisser nos recherehes

(1) Terquem et Jourdy, Bullelin de la sociclé géologique de France, so série, 1, 26, juin 1869. Jote sur le terrain bathonien de la Moselle, p. s63.

C'est ainsi que des monticules marneus dans les environs de Conllans ont été consilérés comme apparlenant a l'oxfordien et classés de la sorte dans le texte de la carte génlorique de la Moselle; leur isolement sur le massif calcairo et h'absence de tout fossile semblaicn: justifier ce rade de classement. Mais lesamen des marnes est venu démontrer, par la présence diue faune microscopigue en tous points iueatique a celle de liontoy, qu'il fallail rapporler ces monticules ḋ loolithe infericure. 
incomplètes pour le bathonien pris en son entier; celles que nous avons tentées pour les zones inféricures et supérieures sont restées presque toutes stériles. Si l'extrême perméabilité des couches n'avail contribué à faire disparaitre la grande majorité de la faune microscopique, nous y aurions certainement trouvé des espèces aussi variées que dans la zone moyenne et nous aurions des données exactes pour les diverses profondeurs des mers de cette époque; nous aurions d'utiles points de repères sur la constitution du bathonien en général, et nous saurions, par la localisation des espéces et leur station, quelle zone est atrophiéc ou quelle est celle qui a reç une extension extraordinairc. Nous pouvons espérer qu'une autre province, plus heureusement dotée que le dépártement de la Noselle, nous donnera ces éléments d'étude qui nous manquent, et viendra combler celle regrettable lacune.

\title{
F O R A II I I F R R S
}

\author{
DE L'OOLITHE INFÉRIEURE
}

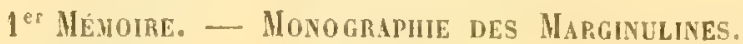

\begin{tabular}{|c|c|c|c|c|c|}
\hline Marginulina & pentagona, & & Marginulina & tumida & Terq. \\
\hline$»$ & acutangularis & » & » & disparilis & $w$ \\
\hline$»$ & subaquilateralis & $\omega$ & 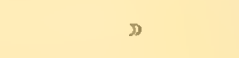 & scalprum & " \\
\hline ) & proxima & $»$ & 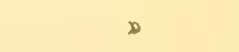 & clausa & » \\
\hline$》$ & inconstans & ” & ” & condita & ” \\
\hline w & accincla & ” & ” & cytharella & » \\
\hline b & inversa & ” & $»$ & flabelloides & $"$ \\
\hline 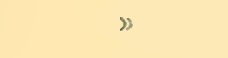 & cornucopia & $\triangleright$ & $\infty$ & macilenta. & $\infty$ \\
\hline b & clathrata & $"$ & ” & heteropleura & $»$ \\
\hline ” & semipartita & $»$ & » & bigibbosa & ” \\
\hline ; & sagittiformis & » & » & pinguis & $\triangleright$ \\
\hline » & pauperala & $\bullet$ & 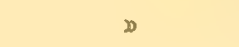 & distensa & 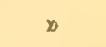 \\
\hline$»$ & protensa & $\varnothing$ & » & solida & $\infty$ \\
\hline$x$ & harpula & 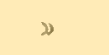 & v & contracta & 2 \\
\hline$\triangleright$ & crustuliformis & b & 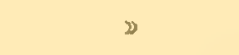 & obstipa & y \\
\hline$》$ & biangulata & $»$ & $n$ & minuta & , \\
\hline
\end{tabular}

2 Mémoire. - Moyographe des Cristellaires.

\begin{tabular}{|c|c|c|c|c|c|}
\hline Crislella & rimordialis, & Terq. & Cristellaria & triquetra & Terq. \\
\hline • & anceps & • & x & paralella & $\nu$ \\
\hline$D$ & guttiformis & , & , & doliolum & $\triangleright$ \\
\hline$\$$ & quadrilateralis & 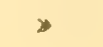 & $\triangleright$ & intorta & \\
\hline
\end{tabular}


Ciristellarin similis

$\begin{array}{ll}" & \text { instabilis } \\ " & \text { subinvoluta } \\ , & \text { suturalis } \\ " & \text { prava } \\ " \quad \text { lagenaformis } \\ " \quad \text { semi-involuta } \\ \text { anomala } \\ \text { leybrida }\end{array}$

Terq.

Cristcllaria centralis

Tero.
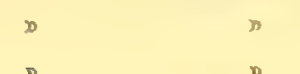

centro-gyrata galeata

inquisita

- helios

$\infty$

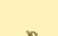

lacunata

- stellaris

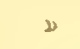

-

- polymorpha

3ै MEMOIRE.

Frondicularia oolithica,

Terq

Ling

spissa
spalulata
lumida
irregularis
longiscata
nodlosaria
dentciliniformis
dolium
cuneata

\%

"

,

(ing

gulina dent

$\nu$

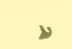

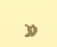

dolium

cordiformis, tetragona

Glandulina turbiniformis alubia

Cornuspira granulosa

\begin{tabular}{|c|c|}
\hline & infraoolithica \\
\hline ע & punctulata \\
\hline 2 & concava \\
\hline D & aspera \\
\hline 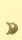 & occlusa \\
\hline
\end{tabular}

Lagena vulgaris

- apiculata

- globosa

„ tenuiaculeata

" agglutinans

Aodosaria

fontinensis

Will.

Reuss.

Walk.

Terq.

\begin{tabular}{|c|c|}
\hline Aodosaria & fontinensis \\
\hline$\nu$ & mutabilis \\
\hline v & agglutinans \\
\hline Dentalina & fontinensis \\
\hline$\infty$ & undulosa \\
\hline » & succineta \\
\hline ע & cuneiformis \\
\hline$"$ & pectinata \\
\hline$»$ & ingens \\
\hline$\nu$ & jurensis \\
\hline D & affinis \\
\hline 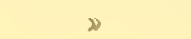 & allernans \\
\hline
\end{tabular}




\begin{tabular}{|c|c|c|c|c|}
\hline Dentalina & intorta & Terø. & Dentaline & juncea \\
\hline » & propinqua & $"$ & " & plebeia \\
\hline 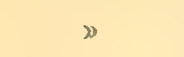 & vermiformis & D & ” & botuliformis \\
\hline ” & clavula & $»$ & w & bicornis \\
\hline 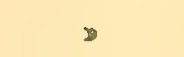 & oolithica & め & Webbina & infraoolithica \\
\hline ১ & cormuformis & . & $»$ & acuminala \\
\hline ఎ & subplana & » & $»$ & flagcllum \\
\hline
\end{tabular}

NotA. Le $44^{\circ}$ mémoire contiendra la description des genres Orbulina, Globigerina, Textilaria, Bulimina, Guttulina, Polymorphina, Spiroloculina, Triloculina et Quinqueloculina.

\section{AMORPHOZOAIRES.}

Les fossiles de cette famille sont en général fort rares dans les terrains ne comprenant que des calcaires marneux et des marnes.

Nous possédons des différentes couches de Fontoy des spicules aciculaires el siliceux qui appartiennent à des spongiaires; nous avons vainement cherché les spicules en étoile, qui, dans les éponges vivantes, accompagnent les spicules en aiguille.

\section{Hipplanus hamlififenus, Lamour. sp.}

Spongia mamillifera, Lamouroux. Exp. meth. des Polyp., p. 88, pl. 84, fig. 11. Hippalimus mamilliferus, d'Orb. Prod. t. 1, p. 325, $\mathrm{n}^{*} 503$. Bathonien. Le tissu de ces spongitaires est plus ou moins grossier; parfois, les cloisons sont très-minces et les mailles microscopiques.

Localité : les Clapes; fort rare. 


\section{REDETOIRE GIVEAL}

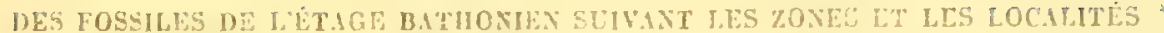

Gaune do lia zome des 1 mmonites suburcutus (Niortensis),

Comprenant ces localités: Côte de Sey, vallée de Montvaux, Amanvillers, Hayange, languevaux, Longwy, Romain, Cons-la-Grandville, Moutigny-sur-Chiers, Jaumont, Gravelotte (calcaire de Jaumont).

TABLEAU N $\quad 1$

\begin{tabular}{|c|c|c|c|c|c|}
\hline tis & $\begin{array}{l}\text { Lstictios } \\
\text { recucillics dins lo zone } \\
\text { Ammoniles subfurcatus. }\end{array}$ & LOCALITE' & 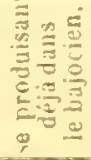 & 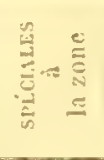 & 等 \\
\hline $\begin{array}{r}7 \\
8 \\
9 \\
10 \\
11 \\
12 \\
13 \\
11 \\
13 \\
11 ; \\
17 \\
18\end{array}$ & 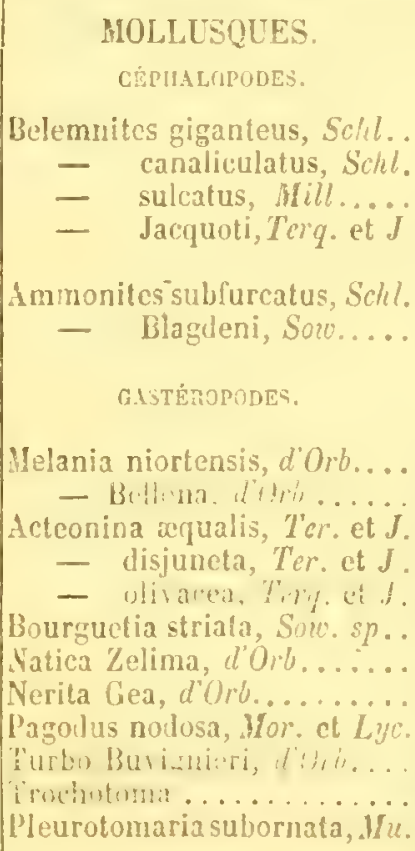 & 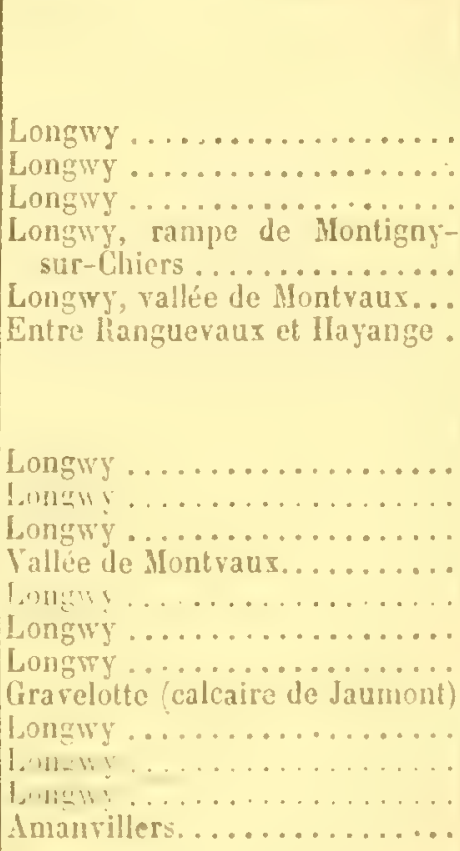 & $\begin{array}{l}0 \\
0 \\
0\end{array}$ & $\begin{array}{c}\cdots \\
\cdots \\
\cdots \\
0 \\
0 \\
\cdots \\
0 \\
0 \\
0 \\
\cdots \\
\cdots \\
0\end{array}$ & $\begin{array}{l}0 \\
0 \\
0\end{array}$ \\
\hline & & 1 reporter..... & 10 & 8 & 4 \\
\hline
\end{tabular}

* L'impression du mémoire était prés d'ètre terminéc, lorsque de nourelles recherches eurent lieu dans quelques localités des environs; il en est résulté de nouvelles indications sur la dispersion de certaines especes qui, nayant pu étre comprises dans le texte, seront consigućcs dinns les tableaux.

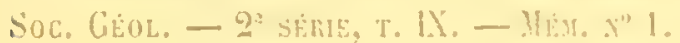


TABLEAU No 1 (SUITE).

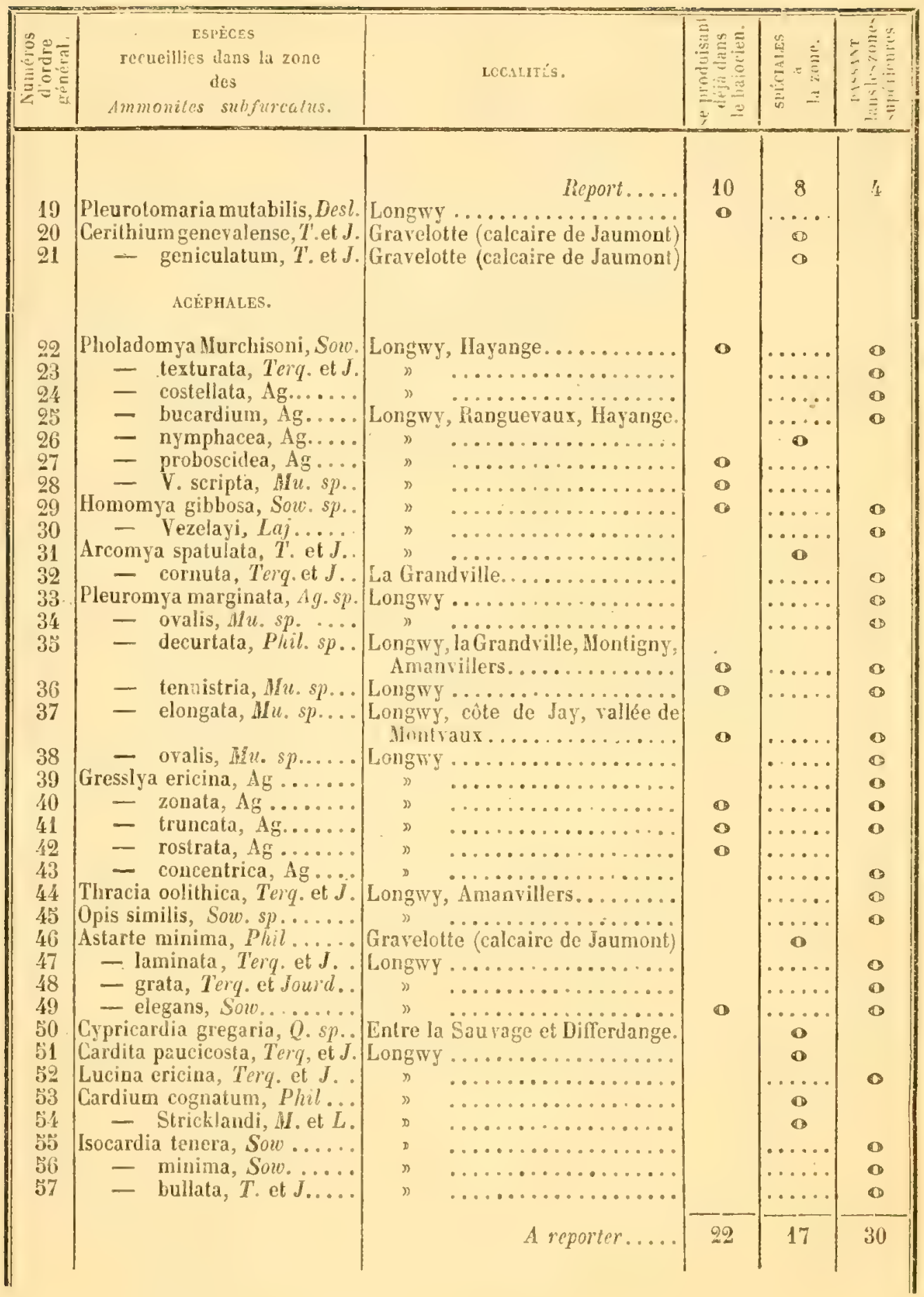


TABLEAU No 1 (SUITE).

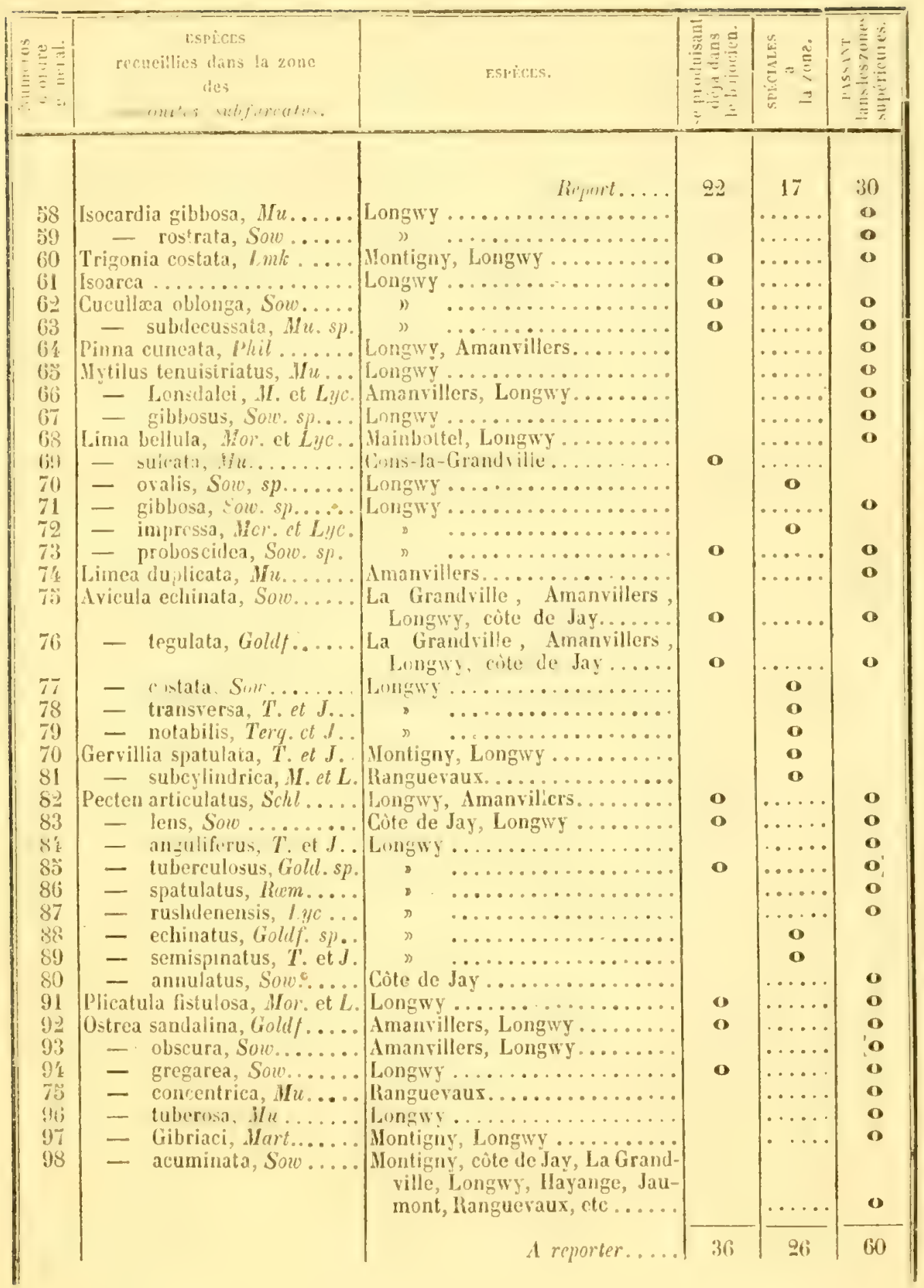


TABLEAU N $N^{\circ} 1$ (SUIte).

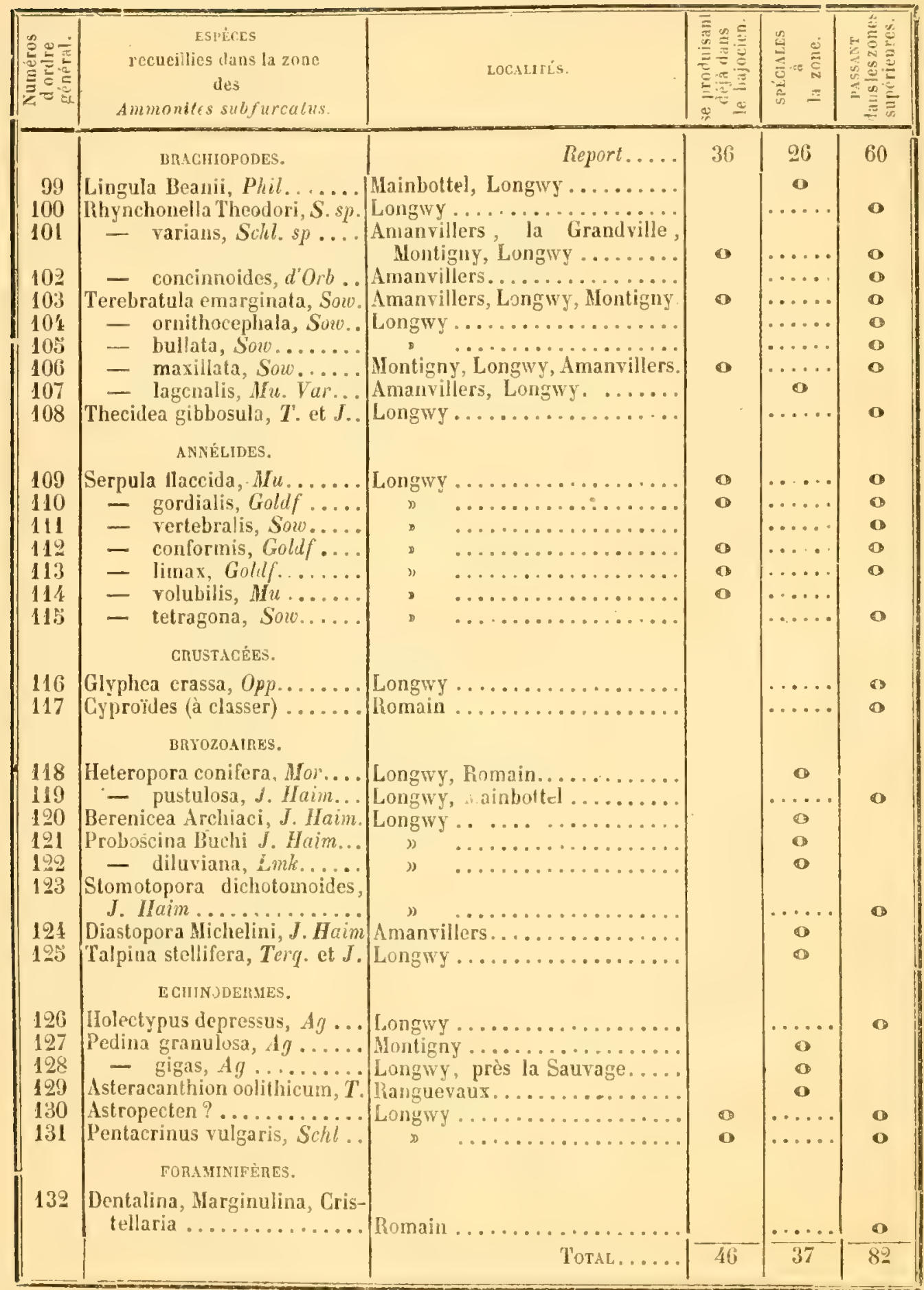




\section{Eanac do Ia zono Ammonites Parkinsoni,}

Comprenant ces localités: Fontoy, Joppécourt, les Clapes, Cutry, Gorze, Gravelotte (marnes), Jarny, rive droite de l'Iron, Yriauville, Thiaucourt, Villey-Saint-Etienne, Angeviller.

TABLEAU NO 2.

\begin{tabular}{|c|c|c|c|c|c|}
\hline $\begin{array}{l}0 \\
0 \\
0\end{array}$ & $\begin{array}{l}\text { espices } \\
\text { recueillies dabs la zone } \\
\text { des } \\
\text { Ammoniles Parlinsoni. }\end{array}$ & LOCaLITIS & 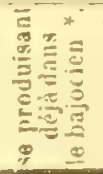 & 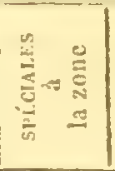 & 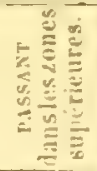 \\
\hline $\begin{array}{l}14 \\
15 \\
10 \\
17 \\
18 \\
19 \\
20 \\
21 \\
22 \\
93 \\
24\end{array}$ & 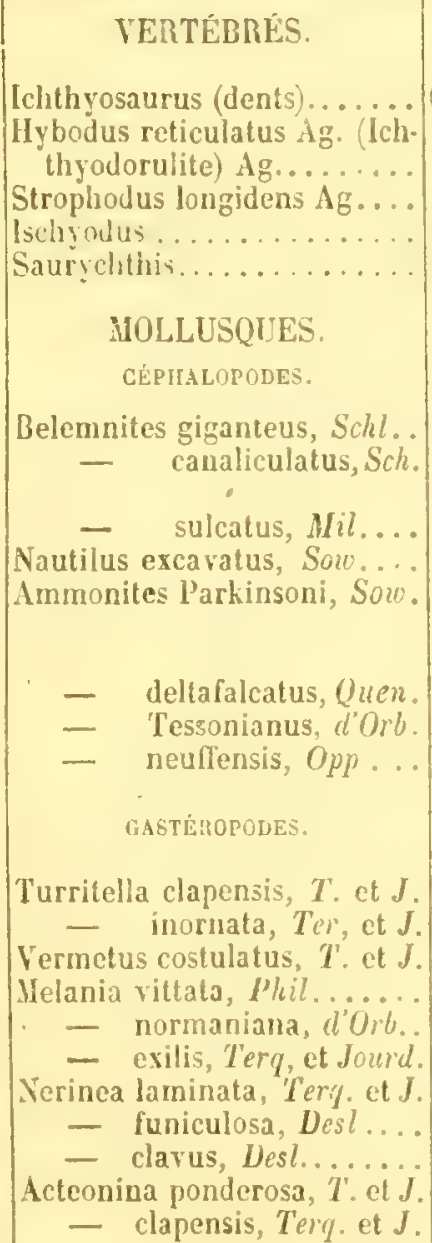 & 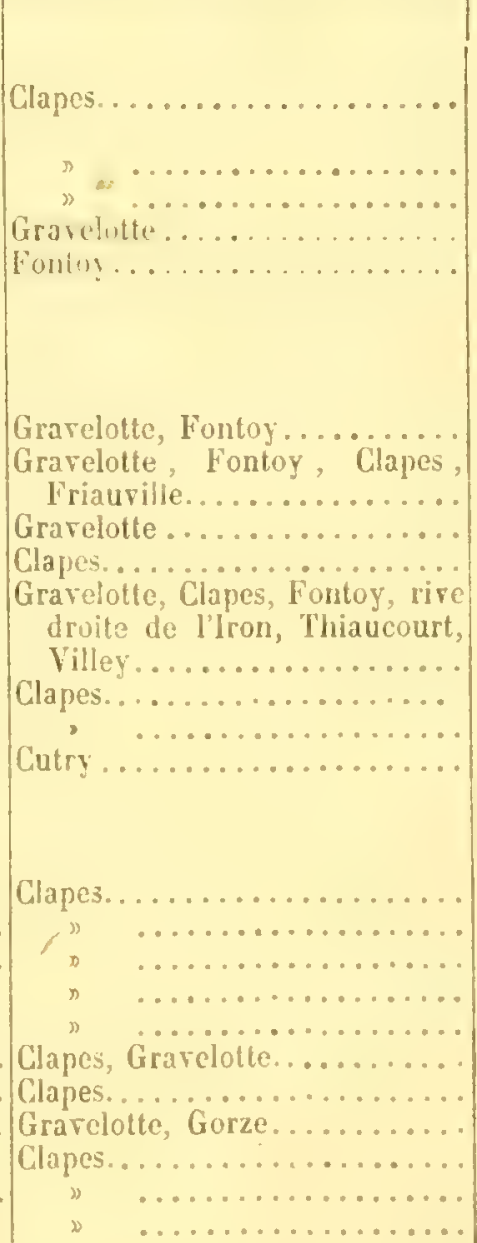 & $\begin{array}{l}0 \\
0 \\
0\end{array}$ & $\begin{array}{l}0 \\
0 \\
0 \\
0 \\
0 \\
0 \\
0 \\
0 \\
0 \\
0 \\
0\end{array}$ & 0 \\
\hline & & A reporter..... & \& & 90 & 1 \\
\hline
\end{tabular}


TABLEAU No 2 (SUTE).

\begin{tabular}{|c|c|c|c|c|c|}
\hline 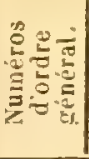 & $\begin{array}{l}\text { CSPL̀ces } \\
\text { recueillies dans la zone } \\
\text { des } \\
\text { Ammonites Parkinsoni. }\end{array}$ & LGCAIITÉs. & 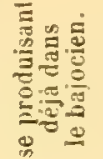 & 造 & 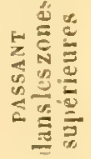 \\
\hline $\begin{array}{l}25 \\
26 \\
97 \\
28 \\
29 \\
30 \\
31 \\
32 \\
33 \\
34 \\
35 \\
36 \\
37 \\
38 \\
39 \\
40 \\
41 \\
42 \\
43 \\
44 \\
45 \\
46 \\
47 \\
48 \\
49 \\
50 \\
51 \\
52 \\
53 \\
54\end{array}$ & 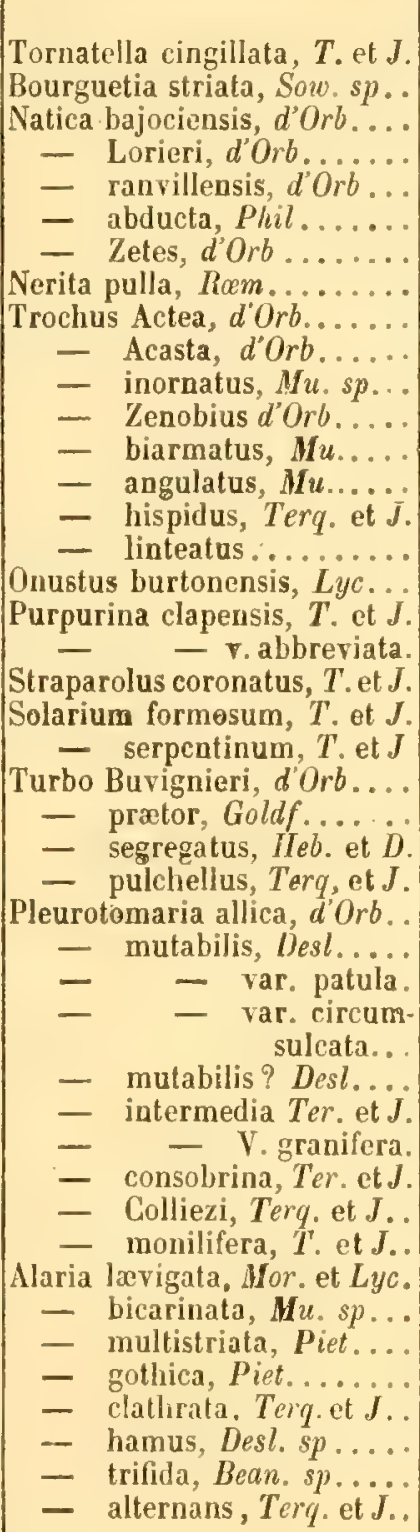 & 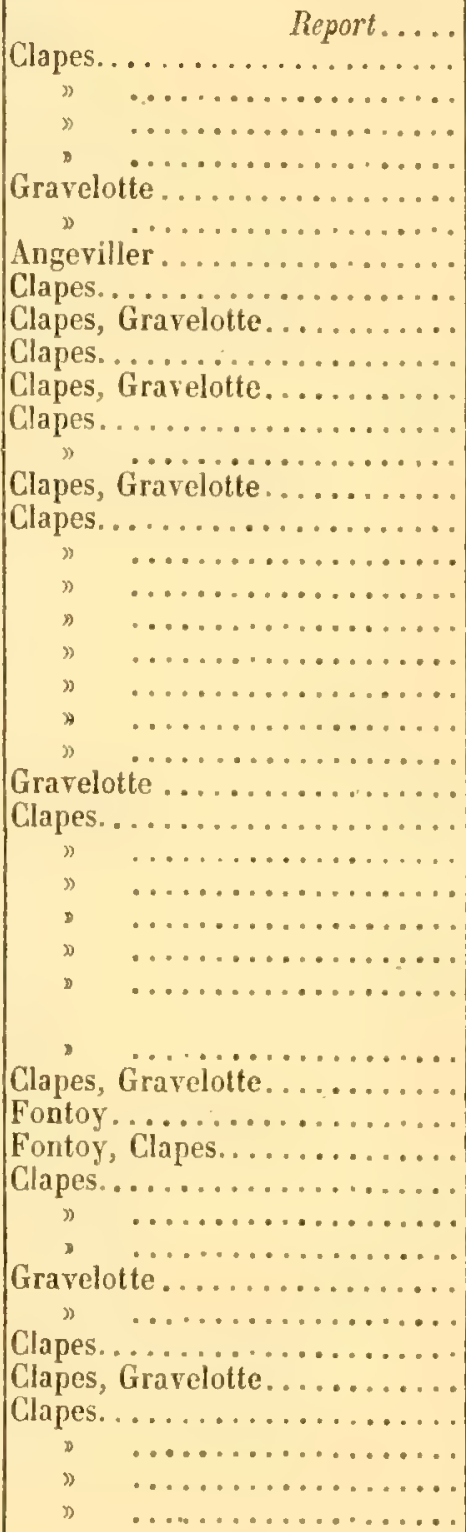 & $\begin{array}{l}\mathbf{0} \\
\mathbf{0}\end{array}$ & $\begin{array}{c}20 \\
0 \\
\ldots \\
0 \\
0 \\
0 \\
0 \\
0 \\
0 \\
0 \\
0 \\
0 \\
0 \\
0 \\
0 \\
0 \\
0 \\
0 \\
0 \\
0 \\
0 \\
0 \\
0 \\
\ldots \\
0 \\
0 \\
0 \\
0\end{array}$ & 1 \\
\hline & & & 8 & 59 & 1 \\
\hline
\end{tabular}


TABLEAU № 2 (SCITE).

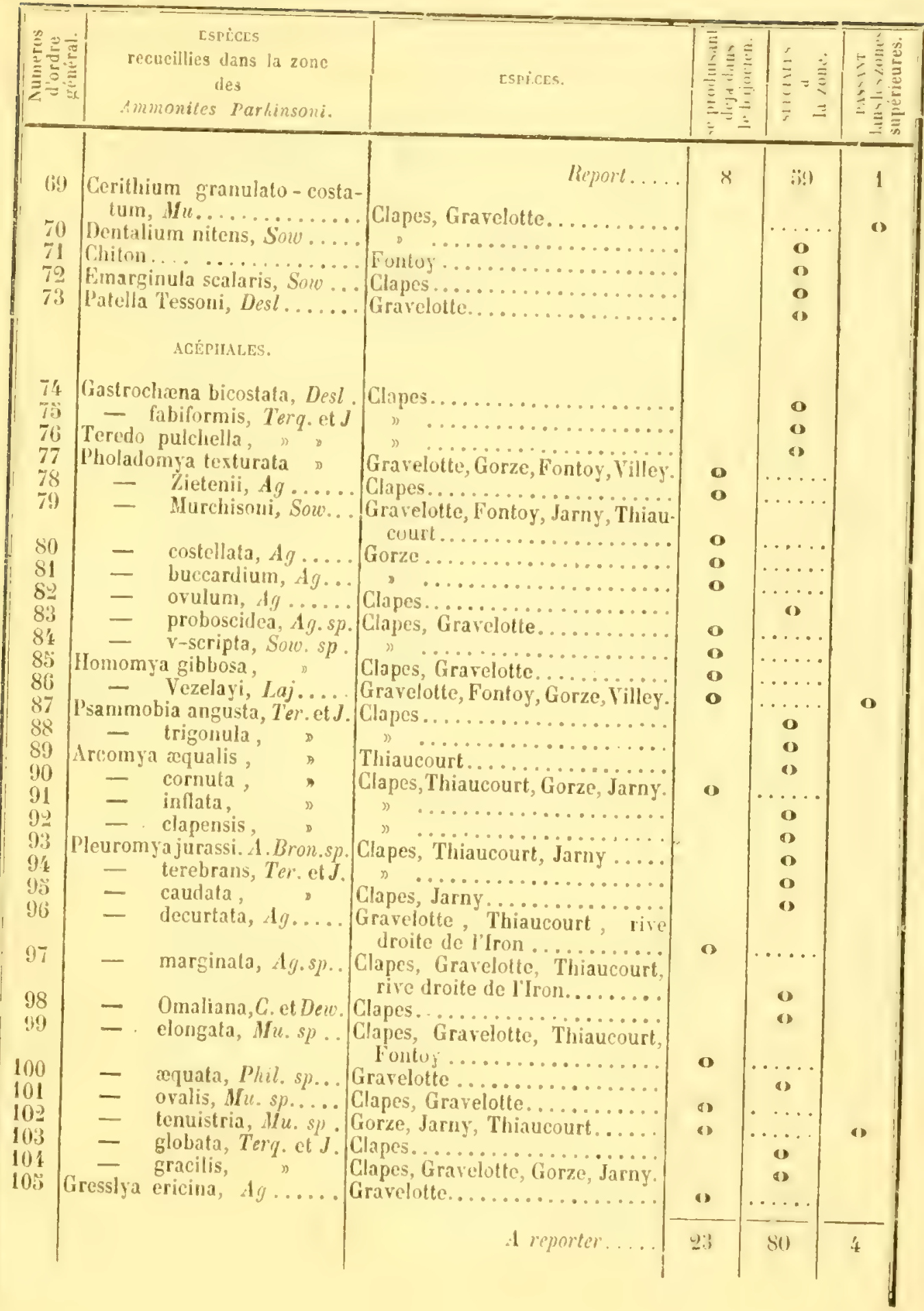


TABLEAU No $N^{\circ}$ (SUITE).

\begin{tabular}{|c|c|c|c|c|c|}
\hline | & 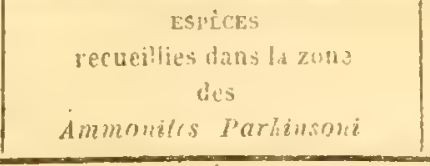 & LOCAMTLS. & $\begin{array}{ll}0 \\
0\end{array}$ & 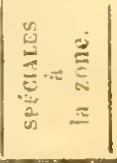 & 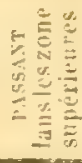 \\
\hline $\begin{array}{l}109 \\
110 \\
111 \\
119 \\
113 \\
114 \\
115 \\
116 \\
117 \\
118 \\
119 \\
120 \\
121 \\
192 \\
193 \\
121 \\
195 \\
126 \\
127 \\
128 \\
129 \\
130 \\
131 \\
132 \\
133 \\
131\end{array}$ & 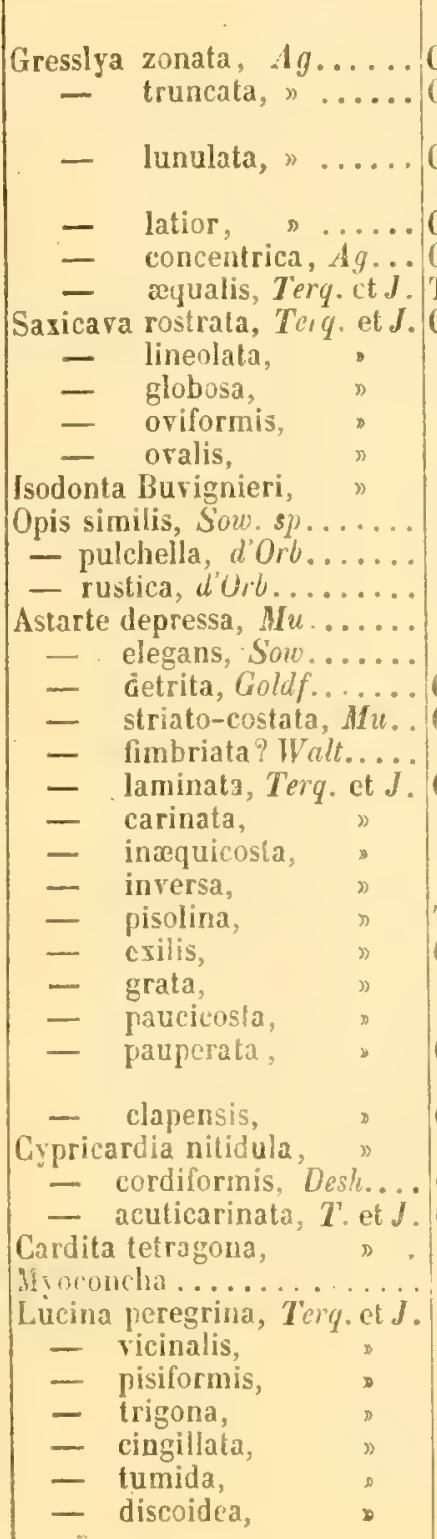 & 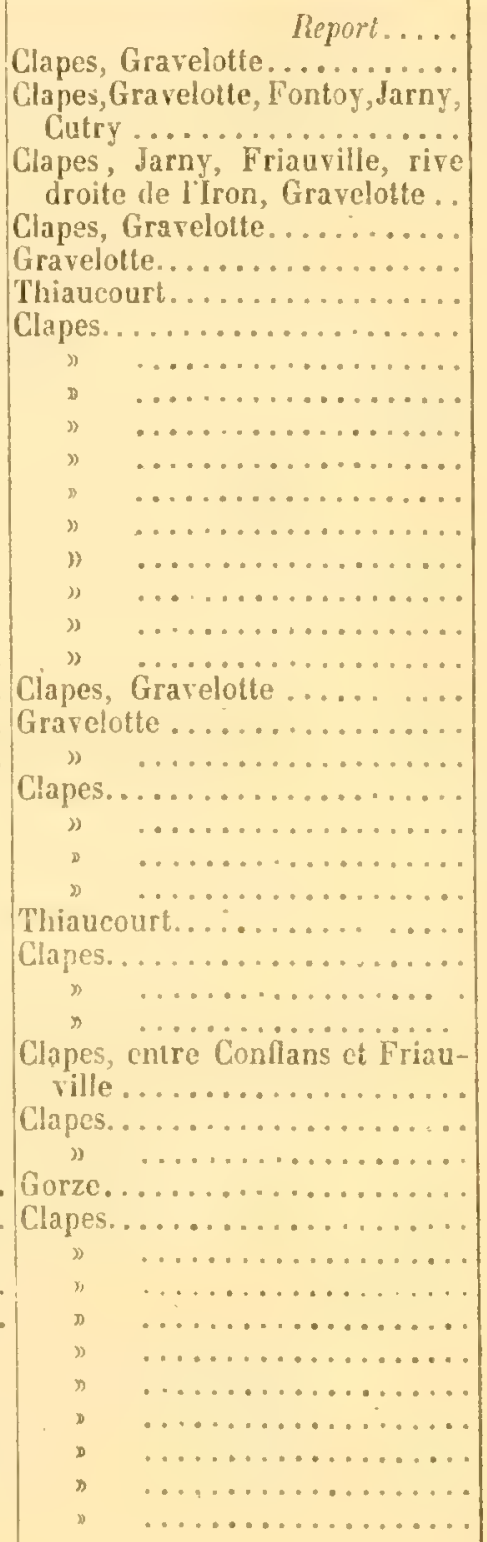 & $\begin{array}{c}23 \\
0 \\
0\end{array}$ & $\begin{array}{c}\ldots \\
0 \\
\ldots \\
0 \\
0 \\
0 \\
0 \\
0 \\
0 \\
0 \\
\cdots \\
0 \\
0 \\
0 \\
\cdots \\
0 \\
0 \\
0 \\
\cdots \\
0 \\
0 \\
0 \\
0 \\
0 \\
\cdots \\
0 \\
0 \\
0 \\
0 \\
\cdots \\
0 \\
0 \\
0 \\
0 \\
0 \\
0 \\
0 \\
0 \\
0 \\
0\end{array}$ & 0 \\
\hline & & & 31 & 113 & 5 \\
\hline
\end{tabular}


TABLEAU No $N^{\circ}$ (SUITE).

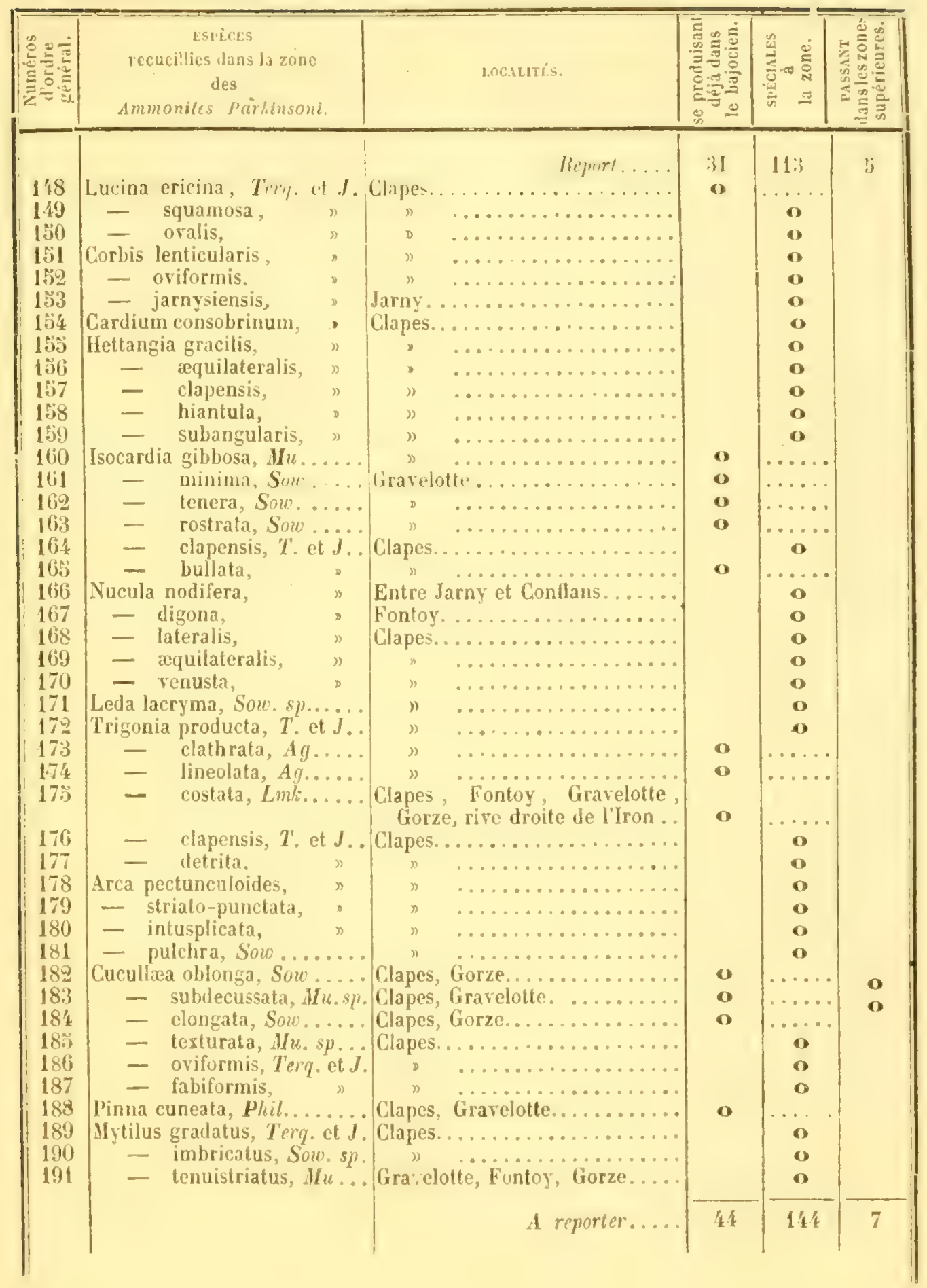


TABLEAU No 2 (suite).

\begin{tabular}{|c|c|c|c|c|c|}
\hline 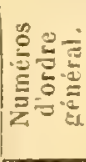 & $\begin{array}{l}\text { ESPÉCEs } \\
\text { recueillies dans la zone } \\
\text { des } \\
\text { Ammonitcs Parkinsoni. }\end{array}$ & Locactis. & 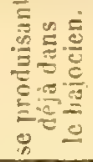 & 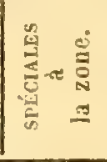 & 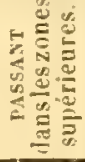 \\
\hline & & Report..... & 44 & 144 & 7 \\
\hline 192 & Mytilus asper, Sow, sp...... & Clapes........................... & & : & \\
\hline 194 & - inclusus, & $"$. & o & & \\
\hline 195 & - Lonsdalci, $M I$. et Lyc. & Clapes, Gravelotte...... & 0 & & \\
\hline 196 & - giganteus, Quenst.sp. & Clapes... ........... & o & & \\
\hline 197 & - Sowerbyanus, S. $s p$. & 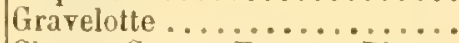 & o & & \\
\hline 198 & - gibbosus, Sow. sp... & $\begin{array}{c}\text { Clapes, Gorze, Fontoy, Pierre- } \\
\text { villers, Gravelotte......... }\end{array}$ & o & & \\
\hline 199 & Lima bellula, Mor. et $L y \mathcal{C}$. & Gorze................... & a & & \\
\hline 900 & - suicata, Mu........ & b $\ldots$ & 0 & & \\
\hline 201 & - notata, Goldt ........ & Thiaucourt...... & & o & \\
\hline 202 & - duplicata, Sow. sp.... & Grarelotte.......... & & 0 & \\
\hline $\begin{array}{l}903 \\
204\end{array}$ & - proboscidea, Sow...... & $" \quad \cdots \cdots \cdots \cdots$ & o & $\because$ & \\
\hline 205 & - tenuistriata, Mu..... & Clapes............. & & o & \\
\hline 206 & - scabrella, Terq. ct J... & 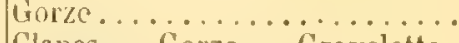 & & $\mathbf{o}$ & \\
\hline 207 & - gibbosa, Sow ........ & 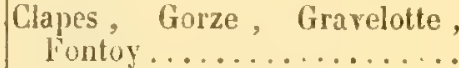 & (D) & & \\
\hline 208 & Limea duplicata, $M u . . .$. & Clapes, Gravelotte, Fontoy..... & 0 & & \\
\hline $\begin{array}{l}209 \\
210\end{array}$ & Avicula digitata Desi. et $J$. & Fontoy, Villers ......... & & 0 & 0 \\
\hline 211 & - echinata, Sow..... & Clapes, Gravelolte, Fontoy & 0 & & 0 \\
\hline 212 & - tegulata, Gold/..... & Clapes, Gravelotte, Fontoy & & & \\
\hline 213 & Gervillia spatulaia, $T$. et $J$. & 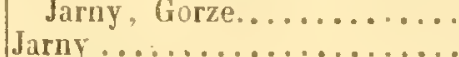 & 0 & & \\
\hline 214 & - lanceolata, Mu... & Gravelotle, Fontoy.... & & 0 & \\
\hline 215 & - acuta, Sow...... & 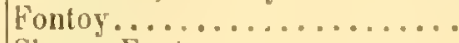 & & o & \\
\hline 216 & - pernoides, Desl... & Clapes, Fontoy........ & & o & \\
\hline 217 & Perna tenuistriata, $T$. et $J$. . & Clapes............... & & $\mathbf{0}$ & \\
\hline 218 & Pecten fibrosus, Sow........ & $\quad \ldots \ldots \ldots$ & & & o \\
\hline 219 & - hemicostatus, M. et $L$. & $"$ & & o & \\
\hline 220 & $\begin{array}{l}\text { - articulatus, Schl..... } \\
\text { - lens, Sow }\end{array}$ & " & o & $\cdots \cdot$ & \\
\hline 221 & - lens, Sow .......... & $\begin{array}{l}\text { Ulapes, Gravelotte, Fontoy } \\
\text { Jarny, Gorze, Thiaucourt... }\end{array}$ & o & & o \\
\hline 222 & - cingulatus $P$ hil..... & 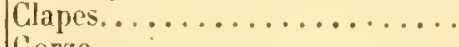 & & o & \\
\hline $\begin{array}{l}223 \\
294\end{array}$ & - anguliferus, $T$. et $J$. & Gorze...$\ldots \ldots \ldots \ldots \ldots \ldots \ldots \ldots$ & a & $\cdots$ & \\
\hline 225 & - anomalus, & Entre Conflans et Friauville, & & & \\
\hline 226 & - tuberculosus, Gold.sp. & 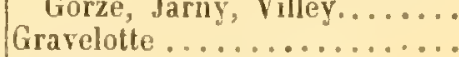 & $\mathbf{O}$ & & o \\
\hline 227 & - rushdenensis, byc... & 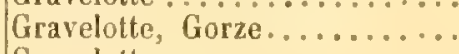 & o & & \\
\hline 228 & spatulatus, $R o c m . . .$. & Gravelotte................ & o & & \\
\hline 229 & - amnulatus, Sow...... & $"$ & o & $\ldots .$. & \\
\hline \multirow[t]{2}{*}{230} & - Germaniæ, Goldf. sp. & & & $\mathbf{o}$ & \\
\hline & & & 64 & 160 & 11 \\
\hline
\end{tabular}


TABLEAU No 2 (sUTE).

\begin{tabular}{|c|c|c|c|c|c|}
\hline 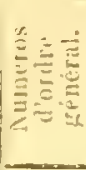 & $\begin{array}{l}\text { Espices } \\
\text { recueillies dans la zone } \\
\text { des } \\
\text { Ammonites Parfinsoni. }\end{array}$ & ESTICES. & 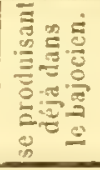 & 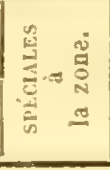 & 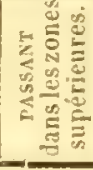 \\
\hline & & Report..... & 64 & 160 & 11 \\
\hline 231 & Pecten limæformis, $T$. et $J$. & Gravelotte..... & & o & \\
\hline $\begin{array}{l}932 \\
433\end{array}$ & Plicatula fistulosa, Mor et $L y c$ & & o & $\cdots \cdot$ & o \\
\hline 233 & $\begin{array}{l}\text { Ostrea sandalina, Goldf..... } \\
\text { _ obscura, Sok...... }\end{array}$ & 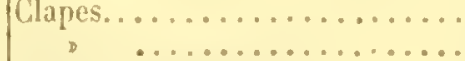 & 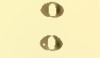 & $\cdots$ & \\
\hline 235 & - gregarea, Sow... & Clapes, Grarelotte... & $\mathbf{0}$ & $\cdots \cdots$ & \\
\hline 936 & - crenata, Coldf. & Clapes............. & o & & \\
\hline 237 & - sulcifera, Phil... & $" \quad \ldots \ldots$ & & o & \\
\hline 238 & - concentrica, .Mu... & "The $\ldots \ldots \ldots$ & o & $\ldots .$. & \\
\hline $\begin{array}{l}339 \\
210\end{array}$ & - tuberosa, Mu..... & $\begin{array}{l}\text { Thiaucourt......... } \\
\text { Cutry, Joppécourt. }\end{array}$ & o & …… & $\mathbf{O}$ \\
\hline 2yi & - Gibriani, Marl.... & Gravelotte, Gorze.... & o & $\cdots \cdots$ & \\
\hline 242 & - acuminata, Sow... & Clapes, Gravelotte, Fontoy. & $\mathbf{o}$ & $\ldots .$. & \\
\hline 213 & - Sowerbyi, Sow sp... & Jarny, rive droite de l'Iron.... & & ..... & o \\
\hline $\begin{array}{l}241 \\
243\end{array}$ & Anomia detrila, Terq. ct $J$. & Clapes, Gravelotte........... & o & $\cdots \cdots$ & \\
\hline & BRAcHopoves. & 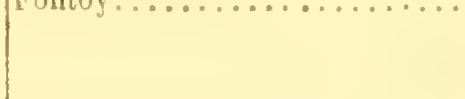 & & 0 & \\
\hline $\begin{array}{l}246 \\
247\end{array}$ & 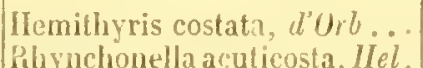 & Clapes, Jarny. ............. & & $\dddot{\ldots}$ & o \\
\hline 218 & $\begin{array}{l}\text { Phiyuchonella acuticosta, Ilel. } \\
\text { - varians, Schl. sp.... }\end{array}$ & 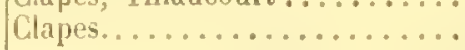 & o & ...... & o \\
\hline 219 & - Therdori. .... & & $\mathbf{o}$ & & \\
\hline 2:in & - comcinu, $S_{1 \mu t}, s p .$. & Jarny $\ldots \ldots \ldots \ldots \ldots$ & & & o \\
\hline 281 & - quadriplicata, Ziet.sp. & $" \quad \ldots \ldots \ldots \ldots$ & & ...... & o \\
\hline $\begin{array}{ll}202 \\
0.023\end{array}$ & - concinnoides, dorb. & Clanes Gravelotte $\cdots$ & & $\cdots$ & $\mathbf{O}$ \\
\hline 200 & $\begin{array}{c}\text { Tercbratula subresupinata, " } \\
\text { - emarginata, Sow.... }\end{array}$ & 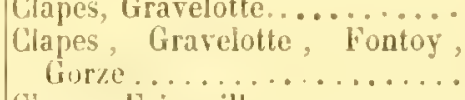 & $\mathbf{O}$ & 0 & o \\
\hline $20 \%$ & - ornithocephala, Zict. & Ciapes, Friauville............ & $\mathbf{O}$ & $\cdots \cdots$ & $\mathbf{o}$ \\
\hline 256 & - maxillata, Sow..... & $\begin{array}{l}\text { Gravelotte, Fontoy, Gorze, Friau- } \\
\text { ville, Jarny ................ }\end{array}$ & o & ... & O \\
\hline 2087 & - bullata, Sow........ & $\begin{array}{l}\text { Gorze, Jarny, Friauville, Thiau- } \\
\text { court... }\end{array}$ & & & \\
\hline 288 & Thecide gibbosula, 7 . et $J$. & Gravelotte.............. & 0 & ...... & 0 \\
\hline & $\begin{array}{c}\text { Crania oolithica, lerq. et } J \text {. } \\
\text { ankwhines. }\end{array}$ & 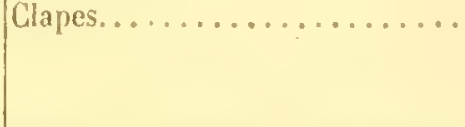 & & o & \\
\hline 260 & Galeolaria socialis, $\mathrm{Br} . .$. & Clapes, Gravelotte, Fontoy.... & $\mathbf{O}$ & & o \\
\hline 261 & Ditrypa fontinensis, $T$. et $J$. & Fontoy $\ldots \ldots \ldots \ldots \ldots \ldots$ & & o & \\
\hline 262 & Serpula flaccida, $\mathbf{M} u \ldots . .$. & Clapes, Gravelotte, Fontoy.... & o & .... & o \\
\hline 263 & - gordialis, Goldf..... & 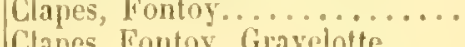 & o & - .... & \\
\hline 263 & $\begin{array}{l}\text { - tetragona, Sow...... } \\
\text { - quadrilalera, Goldf... }\end{array}$ & 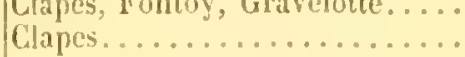 & & $\because$ & \\
\hline \multirow[t]{2}{*}{$260^{\circ}$} & - scobulinu, Terq. et $J$. & & & o & \\
\hline & & & 85 & 169 & 25 \\
\hline
\end{tabular}


TABLEAU $N^{\circ} \cong$ (SUITE).

\begin{tabular}{|c|c|c|c|c|c|}
\hline 至 & $\begin{array}{l}\text { Espèces } \\
\text { recueillies dans la zone } \\
\text { des } \\
\text { Ammonites Parkinsoni. }\end{array}$ & Locautitis & 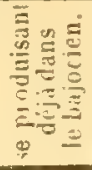 & 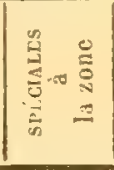 & 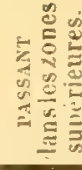 \\
\hline & & Report.... & 85 & 169 & 25 \\
\hline $\begin{array}{l}267 \\
268\end{array}$ & $\begin{array}{c}\text { Serpula vertebralis, Sow... } \\
\text { - tricarinata, }, \ldots\end{array}$ & $\begin{array}{l}\text { Clapes, Fontoy, Gravelotte..... } \\
\text { Clapes.................... }\end{array}$ & & $\cdots$ & \\
\hline 269 & - pentagona, Goldf... & ${ }_{n}^{n} \quad \ldots \ldots \ldots \ldots \ldots \ldots \ldots \ldots \ldots$ & & 0 & \\
\hline 270 & - volubilis, $M u \ldots . .$. & " & o & $\cdots \cdots$ & \\
\hline 271 & 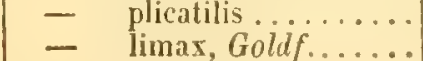 & $\begin{array}{l}\text { Jarny ....................... Friaville } \\
\text { Clapes . }\end{array}$ & $\mathbf{O}$ & & 0 \\
\hline 973 & - conformis, Goldf... & Gravelotte, Jarny, Gorze......... & 0 & $\cdots \cdots$ & 0 \\
\hline & CRUSTacés. & & & & \\
\hline 274 & Glyphea crassa, $O p p . . . .$. & Friauville..... & o & & \\
\hline 275 & Bras de Macroure indétermin. & Fontoy........... & & 0 & \\
\hline 276 & $\begin{array}{c}\text { Cyproïdes (à classer) ...... } \\
\text { BRyozodires. }\end{array}$ & $\quad \cdots \cdots \cdots$ & o & ..... & $\mathbf{o}$ \\
\hline 277 & Heteropora pustulosa, J. H. & 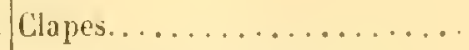 & o & & \\
\hline $\begin{array}{l}278 \\
279\end{array}$ & $\begin{array}{c}\text { Stomatopora Terquemi, } \\
\text { - dichotomoides, }\end{array}$ & Entre Jarny et Conflans, Thiau- & & o & \\
\hline 280 & Diastopora Wrigthi. & 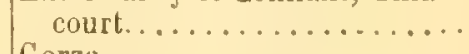 & D & & \\
\hline 281 & $\begin{array}{l}\text { Uiastopora Wrighi, } \\
\text { - scobulina, }\end{array}$ & $\begin{array}{l}\text { Gorze......... } \\
\text { Fontov.... }\end{array}$ & & O & \\
\hline 282 & Berenicea denticulata, $T$. et $J$. & 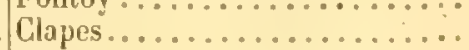 & & o & \\
\hline 283 & Talpina radiciformis, $T e r$, et $J$. & 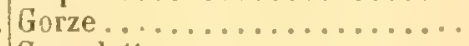 & & 0 & \\
\hline 284 & - latesulcata, " & Gravelotte............. & & o & \\
\hline 285 & $\begin{array}{l}\text { - hyeroglyphica, " } \\
\text { Ecunonerucs. }\end{array}$ & $n \quad \ldots \ldots \ldots \ldots$ & & 0 & \\
\hline 286 & Collyrites ringens, Des Moul.. & 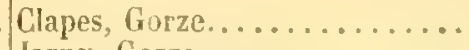 & & o & \\
\hline $\begin{array}{l}287 \\
288\end{array}$ & $\begin{array}{l}\text { Pygurus Michelini, Cot ..... } \\
\text { Clypeus Ploti, Klein }\end{array}$ & 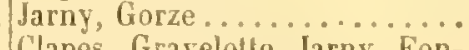 & & o & \\
\hline & 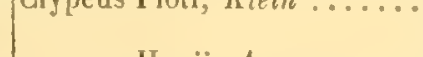 & toy, Thiaucourt, Villey..... & & o & \\
\hline $\begin{array}{l}289 \\
290\end{array}$ & 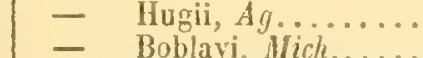 & $\begin{array}{l}\text { Clapes, Gravelotte, Gorze....... } \\
\text { Bive droite de I'Iron. }\end{array}$ & & o & \\
\hline 291 & Echinobrissus clunicularis, $\ddot{C}$. & 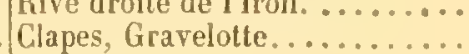 & & 0 & 0 \\
\hline 292 & Holectypus depressus, $A g . .$. & $\Rightarrow \quad \ldots \ldots \ldots \ldots \ldots \ldots$ & o & & $\mathbf{O}$ \\
\hline 293 & Acrosalenia spinosa, " & Gorze, Puzieux .............. & & o & \\
\hline 295 & Diadema complanata, " $\cdots$ & Gorze $\ldots \ldots \ldots \ldots \ldots \ldots$ & & o & \\
\hline 296 & Astropecten? .......... & 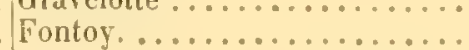 & o & & \\
\hline 297 & Astrophitun? . . . . . . & & & 0 & \\
\hline 398 & Ophiura, Ophiocoma .... & (n. $\quad \ldots \ldots \ldots \ldots$ & & 0 & \\
\hline \multirow{3}{*}{$\begin{array}{l}299 \\
300 \\
301\end{array}$} & $\begin{array}{c}\text { Pentacrinus Dargniesi, } T \text {. et } J \text {. } \\
-\quad \text { vulgaris, } S c h l . . .\end{array}$ & 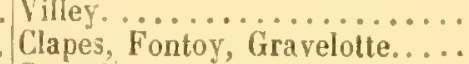 & o & o & \\
\hline & Eugeniacrinus nutans, Goldf. & 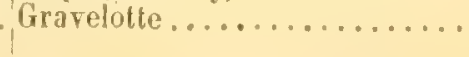 & & o & \\
\hline & & Areporter.... & 96 & 191 & 31 \\
\hline
\end{tabular}


TABLEAU No 2 (SUTE).

\begin{tabular}{|c|c|c|c|c|c|}
\hline 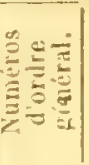 & $\begin{array}{l}\text { EspicLs } \\
\text { recueillies dans la zone } \\
\text { des } \\
\text { Ammonites l'arhinsoni. }\end{array}$ & Locilutis. & 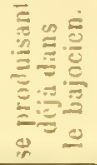 & 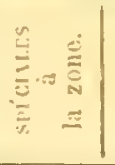 & 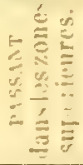 \\
\hline & ZOOPIITES. & Report.... & 96 & 191 & 31 \\
\hline 302 & Gorgonia?........ & 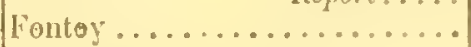 & & 0 & \\
\hline 303 & 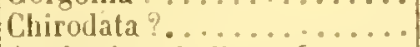 & $\eta_{1} \quad \ldots \ldots \ldots \ldots \ldots \ldots$ & & 0 & \\
\hline 301 & Anabacia orbulites, $L a m$. sp. & Gorze, Auboué, Jarny......... & & 0 & \\
\hline 303 & Montlivaultia trochoides... & Entre Jarny et Conflans...... . & & o & \\
\hline 306 & Isastrea tenuistriata, $M^{\prime} C . s p$. & 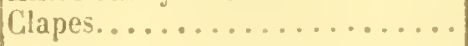 & & 0 & \\
\hline 307 & - Conybeari, Edw. et $M$. & $n \quad \ldots \ldots \ldots \ldots \ldots \ldots$ & & 0 & \\
\hline 308 & - limilata, Lnti. sp.... & ${ }^{\prime} \cdot \ldots \ldots \ldots \ldots \ldots \ldots$ & & 0 & \\
\hline 309 & - serialis, Edw. etII aim. & ${ }_{n} \quad \ldots \ldots \ldots \ldots \ldots \ldots$ & & 0 & \\
\hline 310 & $\begin{array}{c}\text { Thamnastrea Defranciana, } \\
\text { FonAMnIFEnEs. }\end{array}$ & $\ldots \ldots \ldots \ldots \ldots \ldots$ & & a & \\
\hline 311 & Harginulina (32 especes).... & Fiontoy ................... & 0 & $\ldots \ldots$ & 6 \\
\hline 312 & Cristeliaria (2j especes)..... & $\quad \quad \ldots \ldots \ldots \ldots \ldots \ldots$ & 0 & $\ldots \ldots$ & 0 \\
\hline 313 & Frondicularia ( 10 especes).. & $\ldots \ldots \ldots \ldots \ldots \ldots$ & & $\ldots . .$. & 0 \\
\hline 31.4 & Flabellina (17 espèces) ..... & $\ldots \ldots \ldots \ldots \ldots \ldots$ & & 0 & \\
\hline 315 & Haplophragmium (2 especes) & $\ldots \ldots \ldots \ldots \ldots \ldots$ & & 0 & \\
\hline 316 & Lingulina (4. espèces)....... & $\ldots \ldots \ldots \ldots \ldots \ldots$ & & $\mathbf{o}$ & \\
\hline 317 & Glandulina (2 espèces)...... & $\ldots \ldots \ldots \ldots \ldots \ldots$ & & 0 & \\
\hline 318 & Cornuspira (6 espèces) ..... & $\ldots \ldots \ldots \ldots \ldots \ldots$ & & $\ldots \ldots$ & 0 \\
\hline 319 & Lagena (3) espèces). ......... & $\ldots \ldots \ldots \ldots \ldots \ldots$ & & 0 & \\
\hline $3 \geq 0$ & Nodosaria (3 especes) ...... & $\ldots \ldots \ldots \ldots \ldots \ldots$ & & $\ldots \ldots$ & 0 \\
\hline 321 & Dentalina ( 20 espèces)...... & $\ldots \ldots \ldots \ldots \ldots \ldots$ & a & $\ldots \ldots$ & o \\
\hline 322 & Webbina 3 especes)....... & $\ldots \ldots \ldots \ldots \ldots \ldots$ & & & \\
\hline 323 & Orbulina (a classer)........ & $\ldots \ldots \ldots \ldots \ldots$ & $\therefore$ & & \\
\hline 321 & Triloculina (a classer)..... & $\ldots \ldots \ldots \ldots \ldots$ & & $\ldots \ldots$ & 0 \\
\hline 323 & Quinqueloculina (à classer).. & $\ldots \ldots \ldots \ldots \ldots$ & & & \\
\hline 326 & Spiroloculina, id. $\quad$.. & $\ldots \ldots \ldots \ldots \ldots \ldots$ & - & $\ldots \ldots$ & o \\
\hline 327 & Polymorphina, & $\ldots \ldots \ldots \ldots \ldots \ldots$ & & $\ldots \ldots$ & 0 \\
\hline 328 & Globulina, & $\ldots \ldots \ldots \ldots \ldots \ldots$ & & $\cdots \cdots$ & 0 \\
\hline $3 \geq 0$ & Guttulina, & $\ldots \ldots \ldots \ldots \ldots \ldots$ & & 0 & \\
\hline 330 & Textilaria, & $\ldots \ldots \ldots \ldots \ldots \ldots$ & & 0 & \\
\hline 331 & Bulimina, $\quad$ id. $\quad$. & $\ldots \ldots \ldots \ldots \ldots \ldots$ & & 0 & 0 \\
\hline $\begin{array}{l}332 \\
333\end{array}$ & $\begin{array}{lll}\text { Robulima, } & \text { id. } \\
\text { Globigering } & \text { id. }\end{array}$ & ${ }_{\cdots \cdots \cdots \cdots \cdots \cdots} \cdots \cdots \cdots \cdots$ & & $\ldots \ldots$ & 0 \\
\hline 331 & $\begin{array}{l}\text { Globigeriua, } \\
\text { Rotalina, }\end{array}$ & $\begin{array}{c}\cdots \ldots \ldots \ldots \ldots \ldots \\
\cdots \ldots \ldots \ldots \ldots \ldots\end{array}$ & & ...... & 0 \\
\hline 335 & Yonionina, & $\ldots \ldots \ldots \ldots \ldots \ldots$ & & $\ldots .$. & 0 \\
\hline 336 & Planorbulina, $\quad$ id... & $\ldots \ldots \ldots \ldots \ldots \ldots$ & & 0 & \\
\hline \multirow[t]{2}{*}{337} & $\begin{array}{c}\text { AMORPIOzOARES. } \\
\text { Ilippalimusmamilliferus, } L . s p\end{array}$ & Clapes............... & & o & \\
\hline & & & 99 & 210 & 41 \\
\hline
\end{tabular}

Pour rendre plus exact le nombre des espreces contenues dans la zone à A. P'arkinsoni, il faudrait ajouter aux chifires 311 les forminifères déterminés et publiés (127 espèces), plus ceux qui sont à classer (approximativement 4 os espèces et 6 especes de Cyproïdesj; cet ensemble donnerait un tolal de 189 espèces.

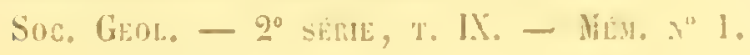


Faume do la zone à Ammonites quercinus,

Comprenant ces localités: Conflans, Béchamps, Thumeréville, Wohécourt, Nouillon-Pont, Joudreville, Viviers, Chenières, Puxe.

TABLEAU No 3.

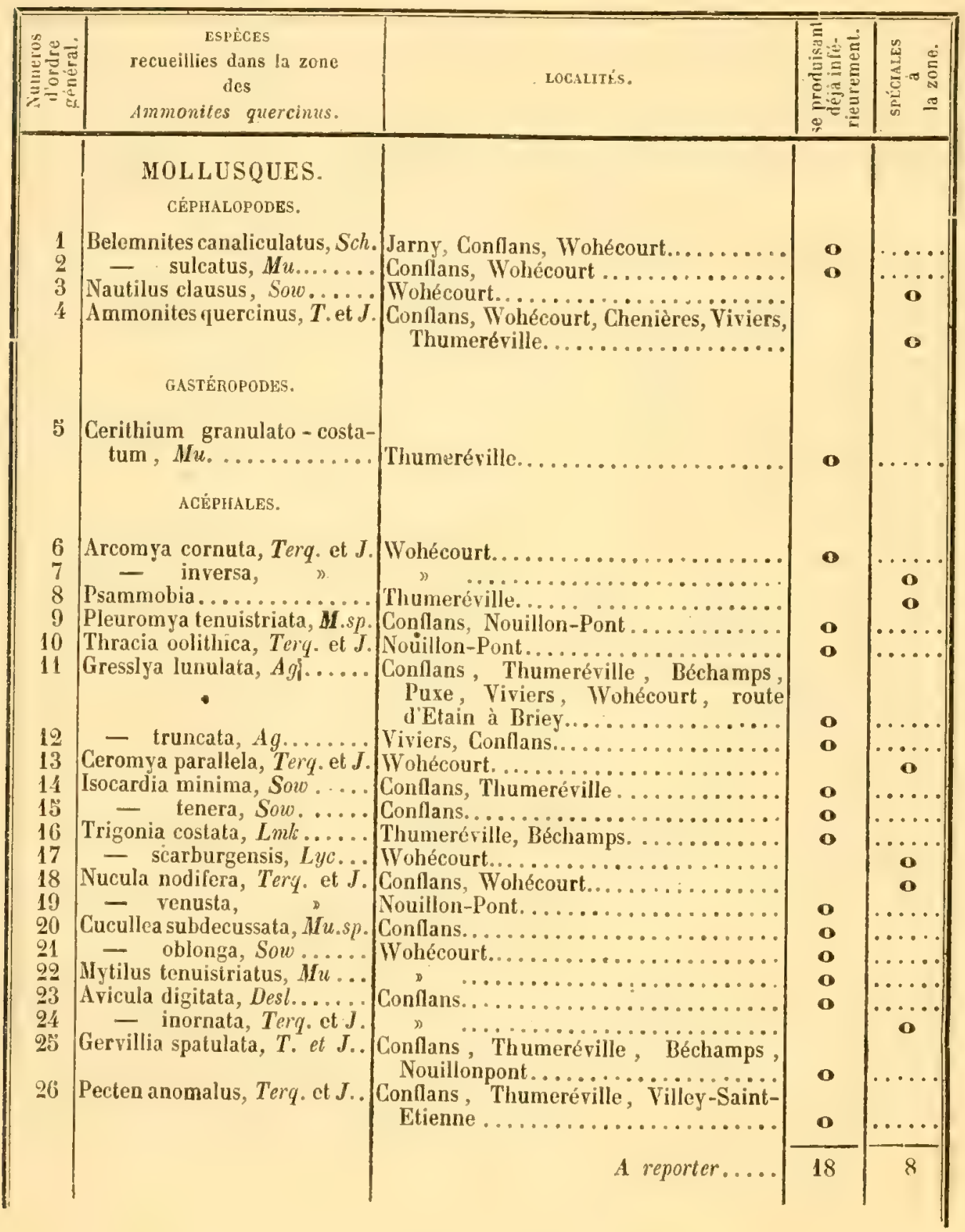


TABLEAU No 3 (sUITE).

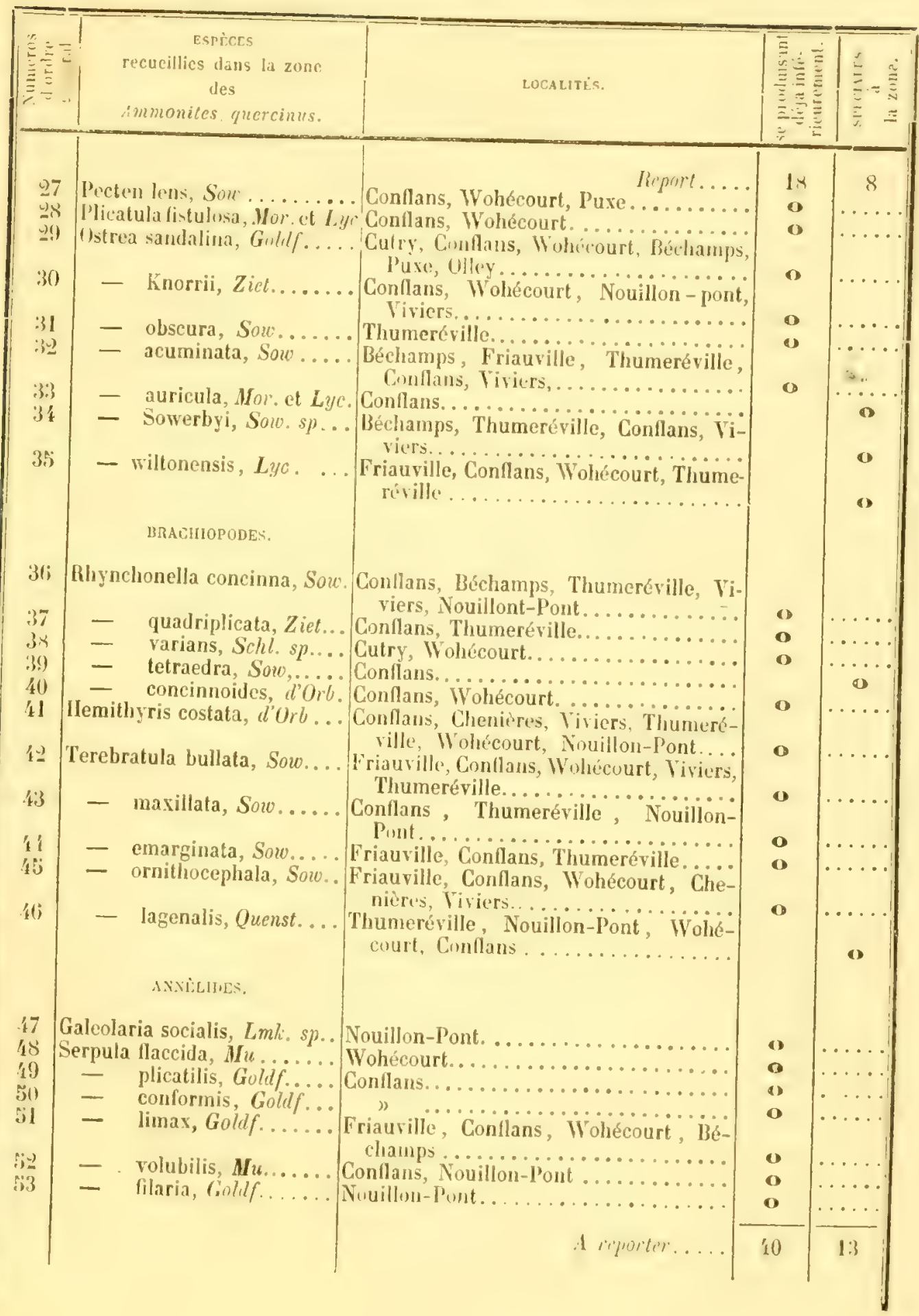


TABLEAU No 3 (SUITE).

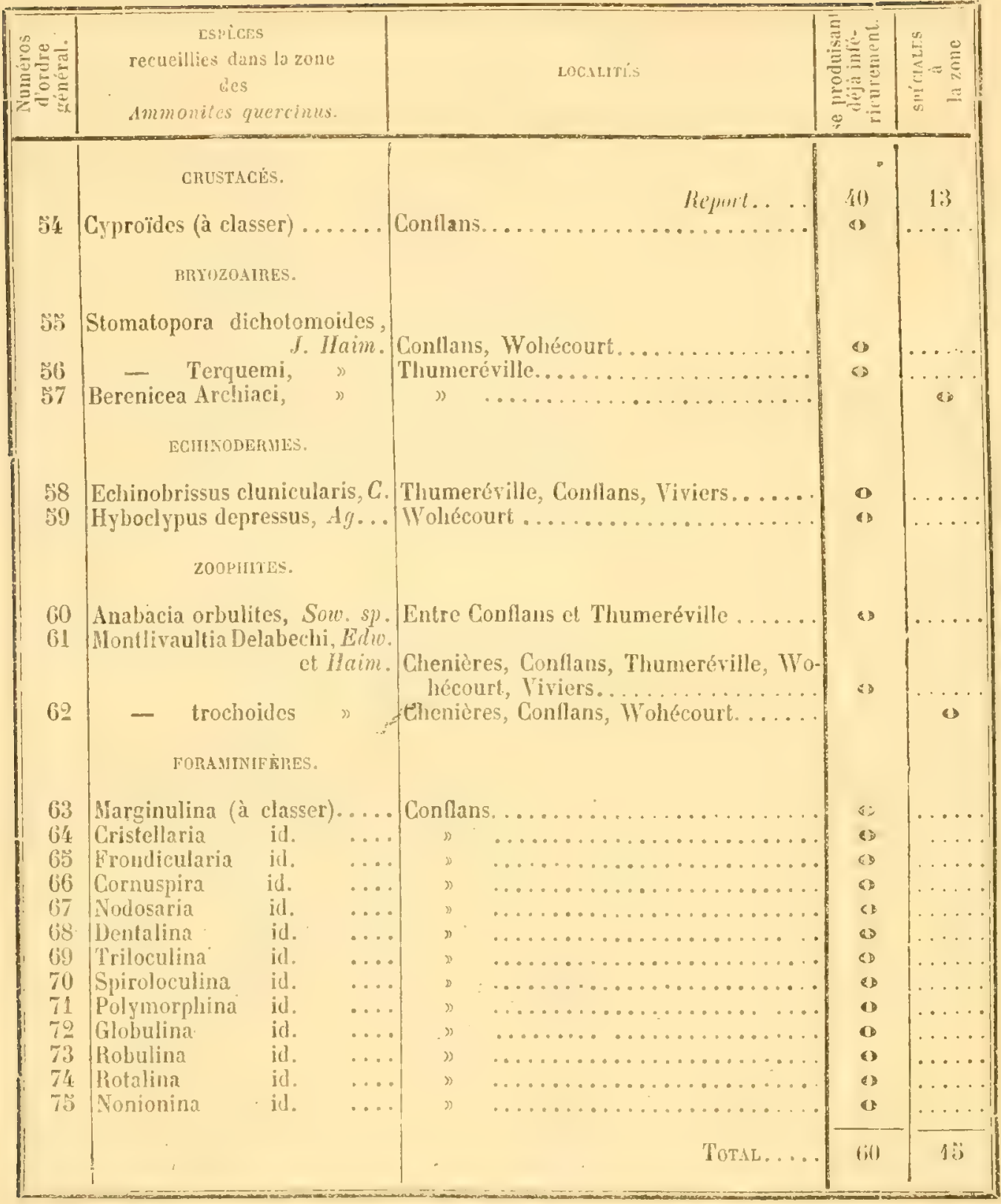




\title{
TABLE ALPHABETIQUE
}

\author{
DES MATIERES
}

\section{PREMIERE PARTIE.} PREMIÈRE SECTION.

Roches de l'étage, sa faune, ses divisions.

$1^{\text {re }}$ zone à A. subfurcatus ...........

ge zone à A. Parkinsoni...........

3 zone à 1. Uurrinus ............

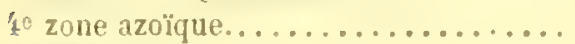

uxfrrdien.

Tableau des couches du bathonien.....

Faune des trois zomes.

\section{IEUXIÈME SECTION.}

Synchronisme de l'étage en Angieterre et dans le nord-est de la France.....

Description géolog. de l'Aisne (d'Archiac)

Statistique géol. de l'Ardennes (Sauvage et Burignier .................

Statistique géol. de la Mleuse (Buvignier)

Synchronisme général ............

Notes sur le gisement des Clapes (Pieite)....................

Ler Mémoire sur les foraminiferes de

l'oolithe (Terquem)..............

Description géologique de la Moselle (Jacquot) Soc. GEOL.

2' SERIE, T. IX.

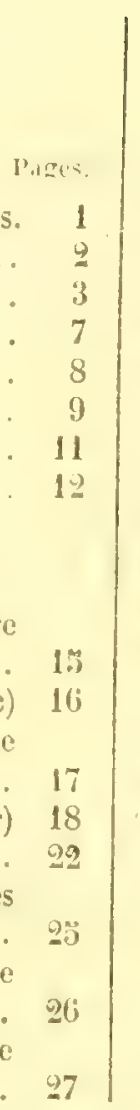

$\sqrt{2}$

DEUXIĖME PARTIE.

PREMIERE SECTION.

$$
\text { l'ages i"! i. }
$$

Introduction à la Paléontologie 30 Aperçu général des corps or-

ganisés............. 33

Vertébrés.............. 33

Mollusques.............. ฏ..

Annélides. .............. 36

Articulés ............. 36

Bryozoaires............ 37

Echinodernes........... 37

Zouphitu............ is

Foraminiliere......... is

Amorphozoaires......... 39

DEUXIÈME SECTHOY.

DESCRIPTIOX DES ESPÈCES . . : औย

ACrosulexis complanata, Ag. 1.14

- spinosa, $\mathbf{A g} \ldots \ldots . .144$

ACTEONINA, dorbigny..... 48

- xqualis, Terq. et Jourd $19 \div$ (i-7

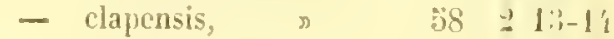

- disjuncta, $491<-10$

- olivacea, \# $\$ 0=11-1=$

- ponderosa, " $\quad$ i0 $=1, j-1 \%$

$\because \cdots$ 
atinte alternans, 1 erd. et J.

- bicarinata, Mu. sp... 66

- clathrata, Terq. et J.. 67 \& 7-8

- gothica, Piet......66 66

- hamus, Desl. sp..... 66

- levigata, Mor. et Lyc. $\mathbf{6 6}$

- Larieri, d'0rb. sp... 66

- multistriata, Piet.... 60

- trifida, liean. sp .... 66

Amonites arbustigerus, d'Orb 'to

- Blagdeni, Sow...... 43

- cordatus, „ ..... 19

- deltafalcatus, Quenst.. 43

- interruptus, Brug.... th

- quercinus, Terq. et J.. ' '

- neuffensis, 0pp..... 43

- niortensis, d'Orb.... 19

- Parkinsoni, Sow..... 49

- subfurcatus, Schl .... 42

- Tessonianus, d'Orb... 43

- wurtembergensis, 0pp. 403

AMPHIDESMa decurtatum, P'ilil. $S 0$

AXABACIA orbulites, Lmk. sp.. 148

- bajociana, d'Orb.....148

. c.sativa Deshaysea, Clap.... 77

Axowid detrita, Terq. et Jourd 13311

- monilifera, „ li,i; 4 6-7

$\therefore: \ldots, \operatorname{Lin} \ldots \ldots \ldots \ldots \ldots, 112$

- intusplicata, Terq. et J. $113127-8$

- pectunculoides, 3 i19 12

- pulchra, Sow...... 11'

- striato-punctata, T.J. 113 1. \$-12

- subdecussata, Mu.... 114

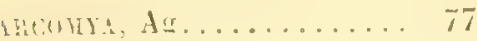

- requalis, Terq. et Jourd 7

- clapensis,

- cornuta,

- inllata,

- inversa,

- spatulata,

ASTARTE cariuata,

- clapensis,

- depressa, Mu....... 9 9

- detrita, finllf....... 9

- elegans, Sow....... 91

- exilis, Terq. et Jourd. 9?

- fimbriata? Walt..... 9

- Irata, Terq. et Jourd.

- insequicostata,
Pagcs. Pl. Fig.

astarte inversa, Terq. et J.. $93 \quad 9 \quad 11$ - 12

- laminata, $\quad$ n 929 (- ;)

- minima, Phil....... 91

- paucicosta, Terq. et J.. 94 9201

- pauperata, " 91920293

- pisolina, " $93918-14$

- striato-costata, Mu... Ml

ASTERACANTHON oolithicum,

Terq. . ....... I's

ASTEROPECTEN . . . . . IS'

Astimphrto........... 14.5 15 12-14

Avicula costata, Sow...... 12.2

- digitata, Desl....... 120

- echinata, Sow...... 120

- inortata, Terq. et Jour. 1231312

- notabilis, $>\quad 1$ : $139-11$

- pectiniformis, Br.... 121

- legulata, Goldf. ..... 120

- transversa, Terq. et J. $122 \quad 138$

BELEKNITES canaliculatus, Sc. 41

- Coquandus, d'0rb.... 12

- giganteus, Schl..... 11

- Bistatus, Btr...... 1

- Jacquoti, Terq. et Jour. \&1 1 (i-9)

- sulcatus, Mil. ...... 41

BEREXICE Archiaci, J. Ilaim. I'근

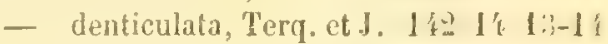

- diluviana, Lmk..... 192

Bounguetia striata, Sow. sp. $\$ 111$ ¿1-2:3

BRACHIURES........... 110

Cardita lunulata, Sow..... 90

- Moreuna, Buv....... 101

- paucicosta, Terq. et J. $96 \quad 10 \quad 23-26$

- problematica, Buv.... 10!

- similis, Sow........ 90

- tetragona, Terq. et J. 97 10 บ-7-

Carduu cognatum, Phil .... 103

- consobrinum, T. et J. 10? $11 \quad 1-3$

- hillanum, Sow...... 10.

- Madridi, d'Arch.... 101

— Stricklandi? Mor. et L. 103

Carpenteria, Desl. ....... 12\%

CERITHUU fusiforme, $\mathrm{H}$. et D. 69

- genevalense, T. et J.. 68 \& 14

- geniculatum, $\quad 68415$

- granulato-costatum, M. 68

- Lorieri, Heb. et Desl. 69

- tortile, » » 69

ceroura ............ Si 
Conges Pl. Fin chemsitzia Bellona, d'Orb.. 47

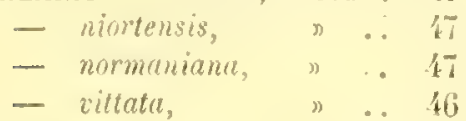

cimiodot............ 1 15 158 8-11

сінто............. 69

eltpeus Boblayi, Mich...... 113

- Inqii, A......... i i.

- patella, А.........143

- Hoti, Klein....... 143

Colsyrites ringuns, Des M. . 14:?

Corris, Cur........... 101

- jarnysiensis, Tery.et J. $10110 \quad 30-38$

- lenticularis, „ 10110 \$1

- Madridi, d'Orb..... 101

- oviformis, Teru. et J. $1021030-33$

согтии, Ag........... 87

(Thisil costata, Terf. ct Jour. 138 11.21-29

critsticés brachiures....... 1'\$0

- ostracodes.......... 110

- palinures ........ 140

GUCULLEA, Link.......... 112

- elongata, Sow....... 11'

- fnbifumis, Terq. et J. 11: 10 18-21

- oblonga, Sow...... 114

- oviformis, Terq. el J. $1141213-17$

- subdecassata, Mu. sp. 114

- texturata, Mu. sp... 11/

Corcamma acuticarinata, ierq. et Jouml..... 9.;

- cordiformis, Desh... 9)

- gregaria, Quenst. sp.. 96

- nitidula, Teri. et Jour. 9ë

ne:ThLllu nitens, Sow.... 69

WADEMA COM Lanatum, Ag.. 19k

DLASTOPORA, Michelini, J. H. 142

- scobulina, J. Hain... I 19

- Wrightii, ” ...142

insasten ringens, $\mathrm{Ag} \ldots \ldots 113$

!TmU1, Berk.......... 138

mTrum fontinensis, T, et J. 138 14 19-20

ichingBRSses clunicularis,

Bl. sp......... 143

marginula scalaris, Sow... 69 ECGEviacrixus nutans, Gold. 147

FORAMATIFERES. . . . . . . . 1:99

vusiat orbulites, Lmk..... 1:12

Galfolata socialis, Br.... 138
9 $28-29$

(2) $30-32$

() $26-27$ 3

(1)

19

38

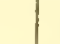

Gastrochena bicostala, Des]. 70

- fabiformis, Terq. et J. 70 \& 20-22

- lacryma, Desl....... 70

- subtrigona, Desl..... 70

Gervilla acuta, Sow..... 12'

- aviculoiles, Goldf... 12'1

- Ianceolata, Mu...... 124

- pernotdes, Desl...... 12t

- siliqua, Den........ iz'

- spatulata, Terq. et J. $12313 \quad 13$

- subcylindrica, M. et L. 12'

Glipheı crassa, 0 pp...... 140

Goxroura proboscidea, Ag... 74

- V-scripta, Goldf.sp.. Th

gongoxit............. 1/47

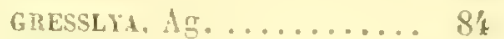

- requalis, Terq. et Jour. S6 8 1-ב

- concentrica, Ag.... 8.j

- cricina, $\mathrm{Ag} . . . \ldots . .83$

- latior, Ag........ 8

- lunulata, Ag....... 8;

- rostrata, Ag........ 8 8i

- truncata, Ag....... 8

- zonata, 4 ........ 8.j

HentTuRis costata, dorb... $13 \%$

Hereropora conifera, Mor... 1/41

- pustulosa, J. Haim... 141

metTaxga, Terq........ 103

- requilateralis, T. et $J .10 \% 118-8$

- clapensis, Terr et Jour. 103 11 6-7

- gracilis, " 10311 4-i3

- Liantula, " 10411 10-11

- subangularis, D $10^{\prime} 111$ 19

IIrNites, Defr. .......... 198

- gradus, Bean. sp.... 190

- tuberculosus, d'Orb... 129

- velatus, d'0rb...... 129

mppotues manilliferus,

Lam. sp........ lii?

holfCtypus deptessus, $\mathrm{Ag} . .143$

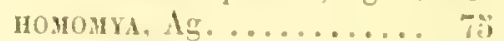

- gibbosa, Sow. sp.... 7j \& $11-1$

- Vezclayi, Laj....... 76

IIYBodes reticulatus, $\mathrm{Ag} \ldots \ldots$ to $10 \quad 1 \quad 3-\mathrm{b}$

ICITYosaunus. .......... 39

ISAstrea Conybeari, Ed. et II. 1:S

- Jimitata, Lmk. sp.... 1.49

- serialis, Edw. cet Haim. 149

- tenuistriata, MCoy.sp. I'S 
Ischrodus, Ag. ......... 40

isodrea, Mu. ........... 111

Isocandia bullata, Terq. et J. $10611 \quad 10 \%-16$

- clapensis, $\quad 10511 \quad 13-14$

- gibbosa, Mu........105

- minima, Sow....... 108

- rostrata, Sow........ 10

- tenera, Sow........ 105

ISOD0NTA Buvignieri, T. et J. 89

- Deshayesea, Buv. .... 89

- Engelhardti, Terq.... 89

- trangularis, Mor. et L. 90

- Woodvardi, „ 90

LED. lacryma, Sow. sp.... 108

Luma bellula, Mor. et Lyc. . $117 \quad 13 \quad$ 1-2

- aardiiformis, Sow. sp. 118

- duplicata, Sow. sp.... 118

- gibbosa, Sorr. sp..... 119

- impressa, Mor. et Lyc. 118

- notata, Goldf....... 117

- ovalis, Sow. sp. .....118

- pectiniformis, M. et L. 118

- pectinoides, Sow. sp. . 118

- proboscidea, Sow.... 118

- scabrella, Terq. et J.. $11913 \quad 3-4$

- sulcata, Hu ........ 117

- tenuistriata, Mu.... 119

LMEA duplicata, Hu. ...... 119

- obtusicosta, Terq. et J. $12013 \quad 5-7$

Livgula Beanii, Sow. . . . . 135

LuenNa, Brug........... 97

- cingillata, Terq. et J.. $99 \quad 10 \quad 10-13$

- discoidea,

$100 \quad 10 \quad 17-18$

- ericina,

$100 \quad 10 \quad 19-20$

- ovalis,

- peregrina.

- pisiformis,

- squamosa,

- trigona,

- tumida,

$100 \quad 1023-94$

$\begin{array}{llll}97 & 10 & 1-2\end{array}$

$\begin{array}{llll}98 & 10 & 5 & -7\end{array}$

$10010 \quad 21-22$

$99 \quad 10 \quad 8-9$

- vicinalis ..........

Lutraile decurtata, Goluf... 83

- jurassi, Al. Brong... 81

- ovalis, Mu........ 83

- tenuistria, Mlu...... 83

Lisianassi, Mu.......... T4

IICT

MELANi Bellona, d'Orb. sp.. 47

- exilis, Terq. et Jourd.. $47 \quad 11 \quad 4-5$
MELAXI niortensis, d'Orb. sp. 47

- normaniana, d'Orb.sp. 47

- striata, Sow........ อัl

- vittata, Phil........ $46 \quad 2 \quad 1-3$

MOdioLd aspera, Sow...... 116

- gibbosa, Sow....... 117

- gigantea, Quenst.... 116

- imbricata, Sow...... 115

- inclusa, Desl....... 116

- parasitica, Sow...... 116

- plicata, Sow....... 116

MONODONTa lavigata, Hu ... 56

Monturvadutia Delabechii,

Edw. et Haim. ... 148

- trochoides, Edw. et H. 148

ma equata, Phil........ 89

- V-scripta, Sow..... T4

MYACITES Kqualus, M. et L. . 8 Z

- jurassi, Quenst..... 81

MYopss jurassi, Ag. ...... 81

- marginata, Ag...... 82

mutudus asper, Sow. sp..... 116

- gibbosus, Sow. sq... 117

- giganteus, Quenst. sp. 116

- gradatus, Terq. et J. . 1151222121

- imbricatus, Sow. sp.. 115

- inclusus, Desl. sp....116

- Lonsdalei, Mor et Lyc. 116

- parasiticus, Desl.sp.. 116

- plicatus, Goldf...... 116

- pulcher, Goldf....... 116

- Sowerbyanus, Sow. sp. $116^{\circ}$

- tenuistrialus, Mu.... 11 כ

matici abulucta, Phil...... ö́t

- bajociensis, d'0rb.... 51 .

- Lorieri d'Orb....... כ็

- pictariensis, d'Orb... כัّ

- ranvillensis, d'Orb... $5 \%$

- Zelima, dorb......

- Zetes, d'0rb........ כั

Nautilus excavalus, Sow.... 42

NERINEA clavus, Dest. ..... 48

- funiculosa, Dest..... 48

- laminata, Terq. et J. $48 \quad$ I 19

verita gea, d'Orb........ 5ั

— pulla, liam........ รั

NUGLEOLITES clumicularis, Bl. 143

xucula xquilateralis, T. et J. $107 \quad 11 \quad 23-28$

- digona, Terr. et Jourd. 10611 21-20 


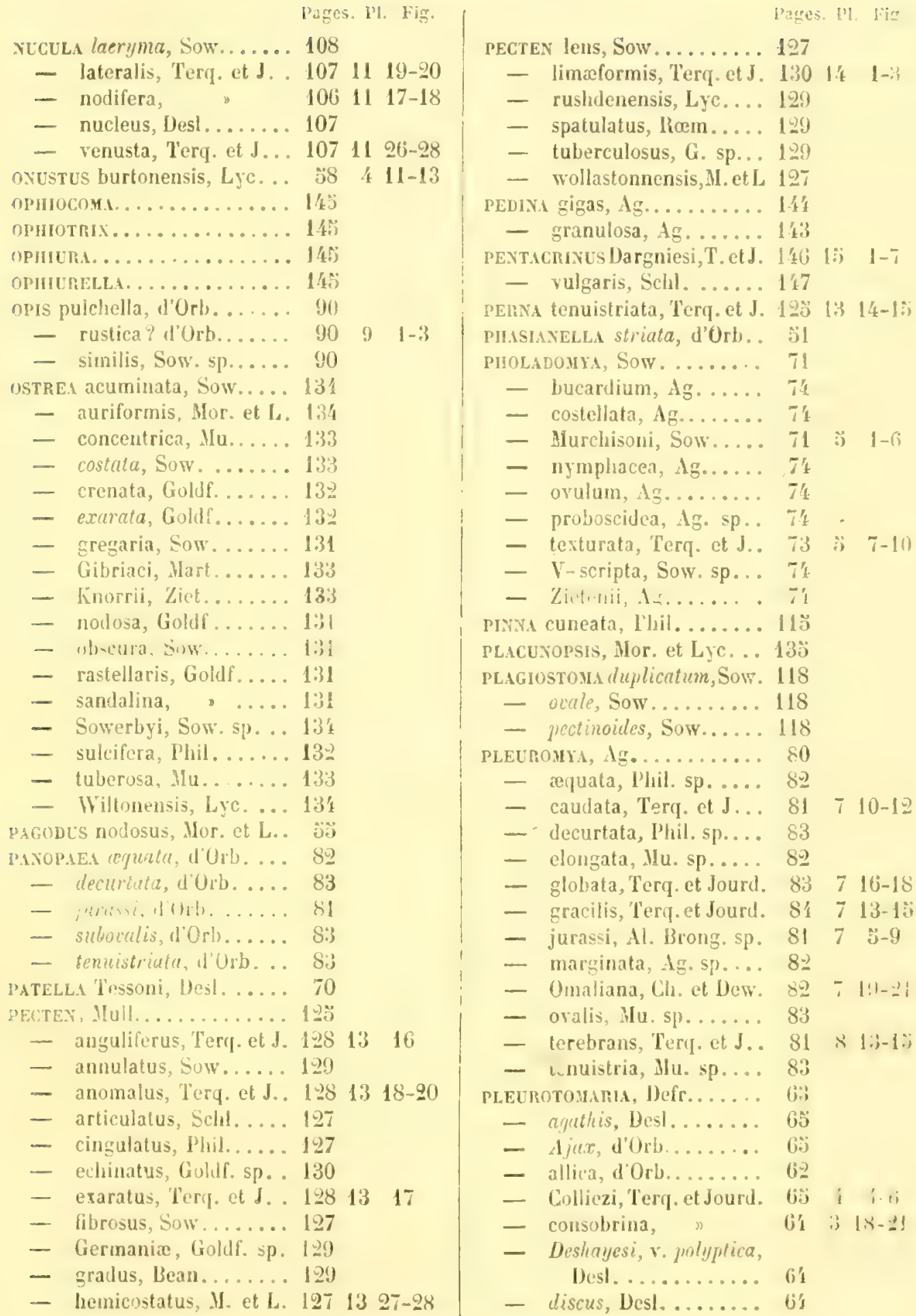


Pages. Pl lirg.

PLEURotomaria granulata, G. 64

- intermedia, Terq. et J. 63 3 13-14

- - var, granifera,

Terq. et J.. $64 \quad 3150-17$

- monilifera, Terf. et J. 63 \& $1 ?$

- montreuilensis, H. et D. 60

- mutabilis, Desl..... 62

- _ var patula...663

- - var. circumsulcata...663

- subornata, Mu..... 62

Plicatuli fistulosa. M. et L. 131

Proboscrixa Buchi, J Haim.. 142

PSAMMORA, Lmk......... 76

- angusta, Terq. et Jour. 76

- trigonula, ə 76

- vespertina......... 76

PTERocera hamus, d Orb.... 66

- Lorieri, dOsb......66

- trifida, d'0rb.......66 $66^{\circ}$

Puruntas clapensis, T. et J.

- - var. abbreviata.... 59

pYGURus Michelini, Cot.... 14 Rostellan bicurinata, Hu. $66^{\circ}$

- hainus, Desl........ 66

- trifida, Bean...... bit

RYYCHONELLA acuticosta, Hel, 135

- concinna, Sow. sp.... 136

- concinnoides, d Orb.. 136

- quadriplicata, Ziet... 136

- Theodori, Schl.sp... 196

- varians, Schl. sp..... 136

salicava globosa, Terq. et J. 88

- lineolata, » 87

- ovalis, $\quad$ " 89

- oviformis,

88

- rostrata,

87

sEnpula conformis, Goldf. . . 110

- Haccida, Hu........ 138

- gordialis, Goldf..... 140

- limax, Goldf....... 140

- pentagona, Goldf. .... 139

- plicatilis, Mu....... 140

- quadrilatera, Goldf... 139

- scobinula, Terq. et J. $1391 \%$ 10-12

- sminlis, liublf...... itis

- tetrazona, Sow..... 139

- tricarinata, Sow. ... 130

$8 \quad 9-10$

$8 \quad j-8$

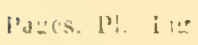

SERPULA verlebralis, Sow . . . 139

- volubilis, Mu....... 139

SOLARIux formosum, $\mathrm{T}$. et $\mathrm{J}, \quad B 0$; $1-3$

- serpentinum, $\quad$ o $6137-7$

SOWERBYa, d'Orbigny...... 90

SPHERA, SOW........... 101

- Madridi, Mor. et Lyc. 101

SPONDyLus tuberculosus, Gold. 129

- velatus, Goldf....... 129

STOMATOPORA T'eryuemi, J.H I'19

- dichotomoiles, J. H,.. 142

strapatroles altus, d'0rb.... 60

- coronatus, Terq. et J. 60033 i-li

strophou us longidens, $\mathrm{Ag} . .440 \quad 1 \quad 1-2$

terebratula bullata, Sow... 137

- emarginata, Sow..... 136

- lagenalis, Sow...... 137

- - var. minor. 137

- maxillata, Sow.....136

- ornithocephala, Sow.. 136

- sibovalis, Sow...... 130

- subresupinata, d'0rb.. 186

2 28-33 TEREDo pulchella, Terq. et J. 70 1 l $18-1 ! 3$

THAMNASTREA Defranciana .

Elw. et Haim.... 119

THecroen gibbisa, Terq. et J. 137 l's i-!?

TURACIA oolithica, „ \&6 \& $11-1=$

TOR.YAtella cingillata, „ $50 \quad 21 ;-20$

Tnigonta cardissa, Ag. .... 109

- clapensis, Terq. et $J . .11011331-i ;$

- clathrata, Ag....... 109

- costata, Lmk....... 109

- detrita, Terq. et Jour. 111 12 1-2

- elonnatu, Sow...... 109

- lineolata, $1 \mathrm{~g} . . . . .103$

- litterata, $\mathrm{Ag} . . . . . . .110$

- producta, T'erq. et J. $10311 \quad 29-30$

- scarburgensiz, II. et L. 111 19:3-4

Trochoto:a, Deshayes.... 6 6

Trocius Acasta, dOrb..... 53

- Actea............

- angulatus, Mu...... \$6

- Buldus d'Orb...... 50

- biarmatus, Mu..... 56

- Brutus, dOrb...... . j8

- helincus, dOrb.....

- hispidus, Terq. et J... 57 2 논.4.;

- inornatus, Mu. sp.... 50

- linteatus, Terg. et J... $58220-1$ 
Tunio segregalus, Heb. et D. 61 TURititela clapensis, T.et J. $\quad$ \&: 1 1: - inornata, Terq. et J. . $16 \quad 1$ 1:i-16 VENCLITES a alensis, (uenst.. 98 virdetes costulatus, T. et J. $16 \quad 1$ 17-18 venMICULARIA nodus, Phil.. . 139)

\section{ABRÉVATIONS DES MOUS D'AUTELRS,}

Ag. ............ Agassiz.

Arch. (d') ....... d'Archiac.

Bean.......... Bean.

Berk. .......... Berkeley.

Blv........... Blainvilie.

Br............ Bronn.

Bron. Al ........ Al. Brongniart.

Brug. . . . . . Bruguires.

Buv. .......... Buvienier.

Chap........... Chapuis.

Chap. et Dew...... Chapuis et Dewalque

Cur. .......... Cuvier.

Desh........... Deshayes.

Dest............ Deslongchamps.

Des II......... Des Moulins.

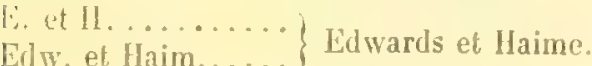

Goldf............ Goldfuss.

I. Haim.......... Jules Haime.

II. et D......... Hébert et

IJeb. et Desl....... Deslongchamps.

IIel. ........... Helmann.

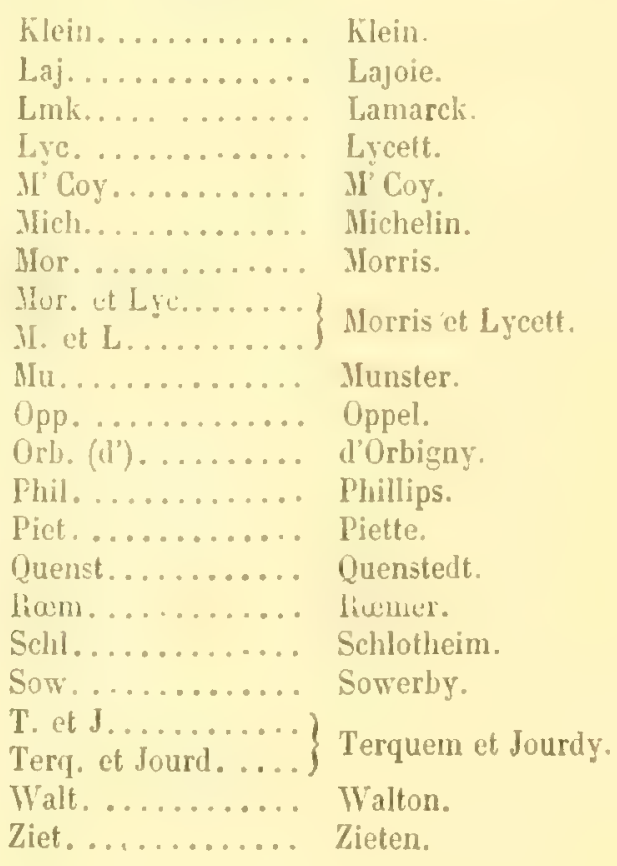

Klein.......... Klein

Isve. ......... Lrcett.

I' Coy.......... II' Coy.

Wich........... Michelin.

Nor. ........... Morris.

II . et L........... W Morris et Lycett.

Mu........... Munster.

Opp........... Oppel.

Orb. (d')........ d'Orbigny.

Phillips.

Pict........ Piette.

Quenst.......... Quenstedt.

hom........... livener.

Schl........... Schlotheim.

Sow........... Sowerby.

T. et J........... Terquem et Jourdy.

Walt........... Walton.

Ziet........... Zieten. 


$$
\text { - }
$$



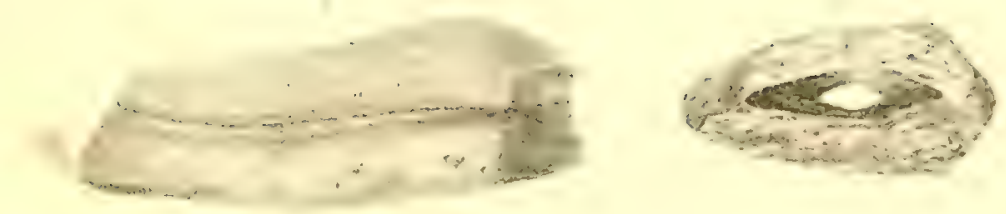

3

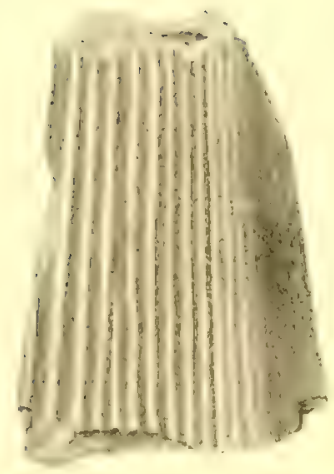

$\frac{1}{7}$
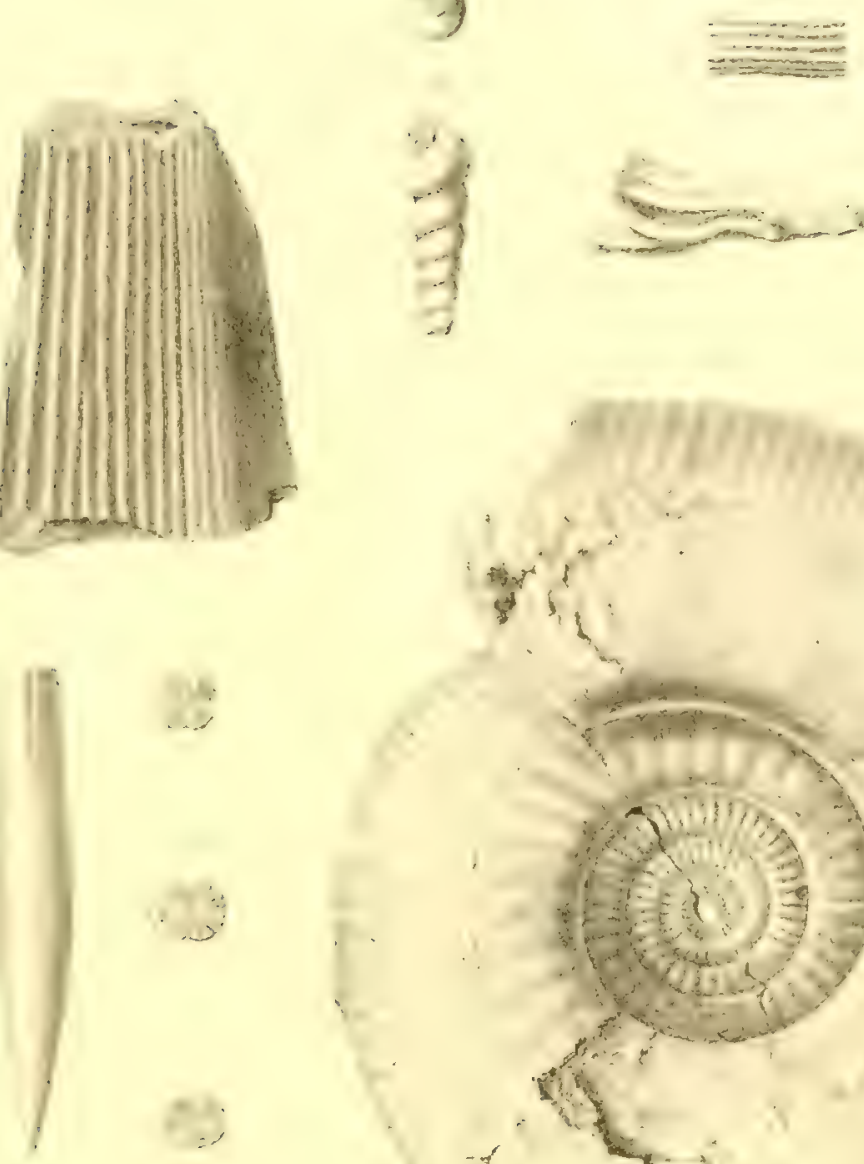
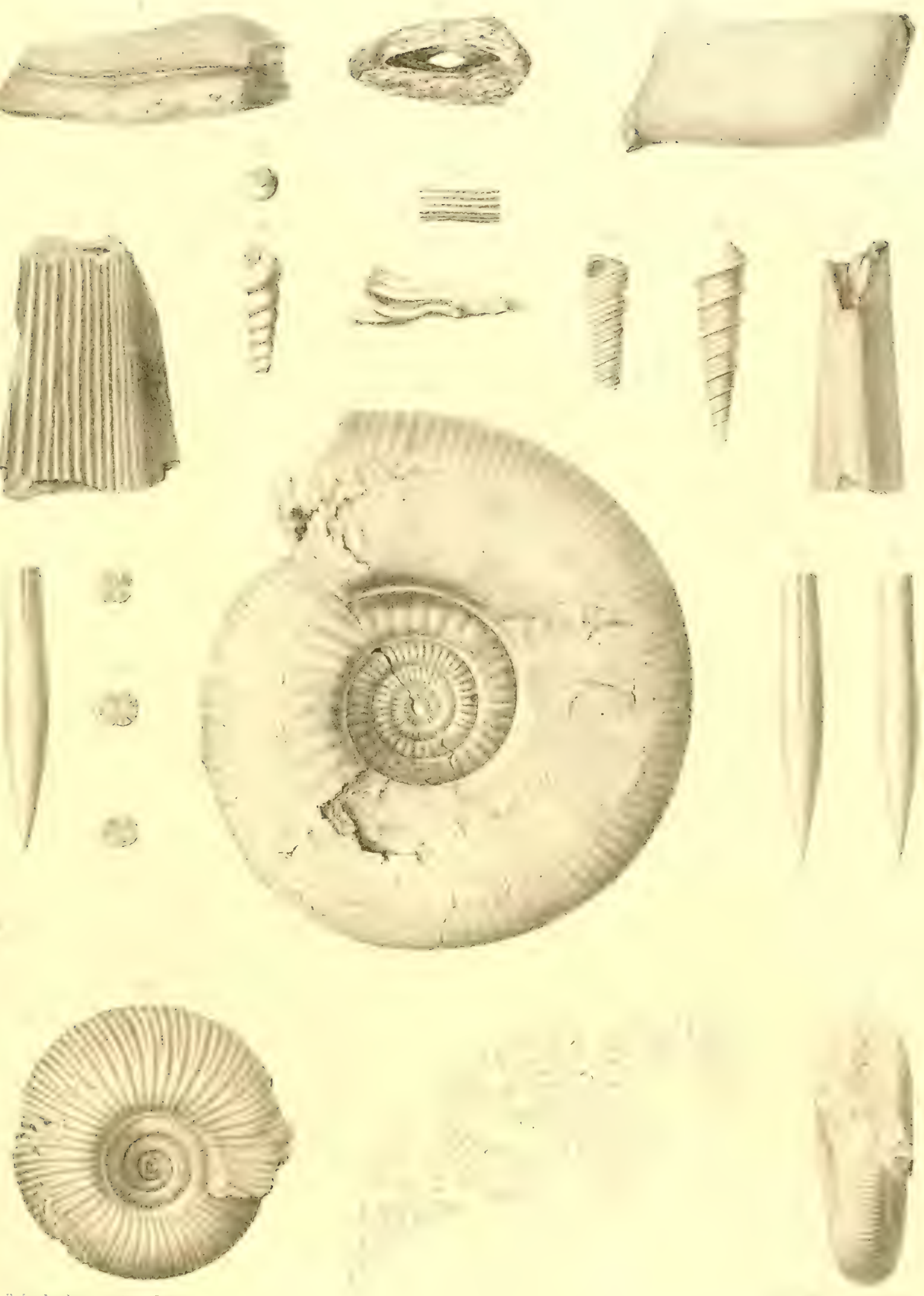


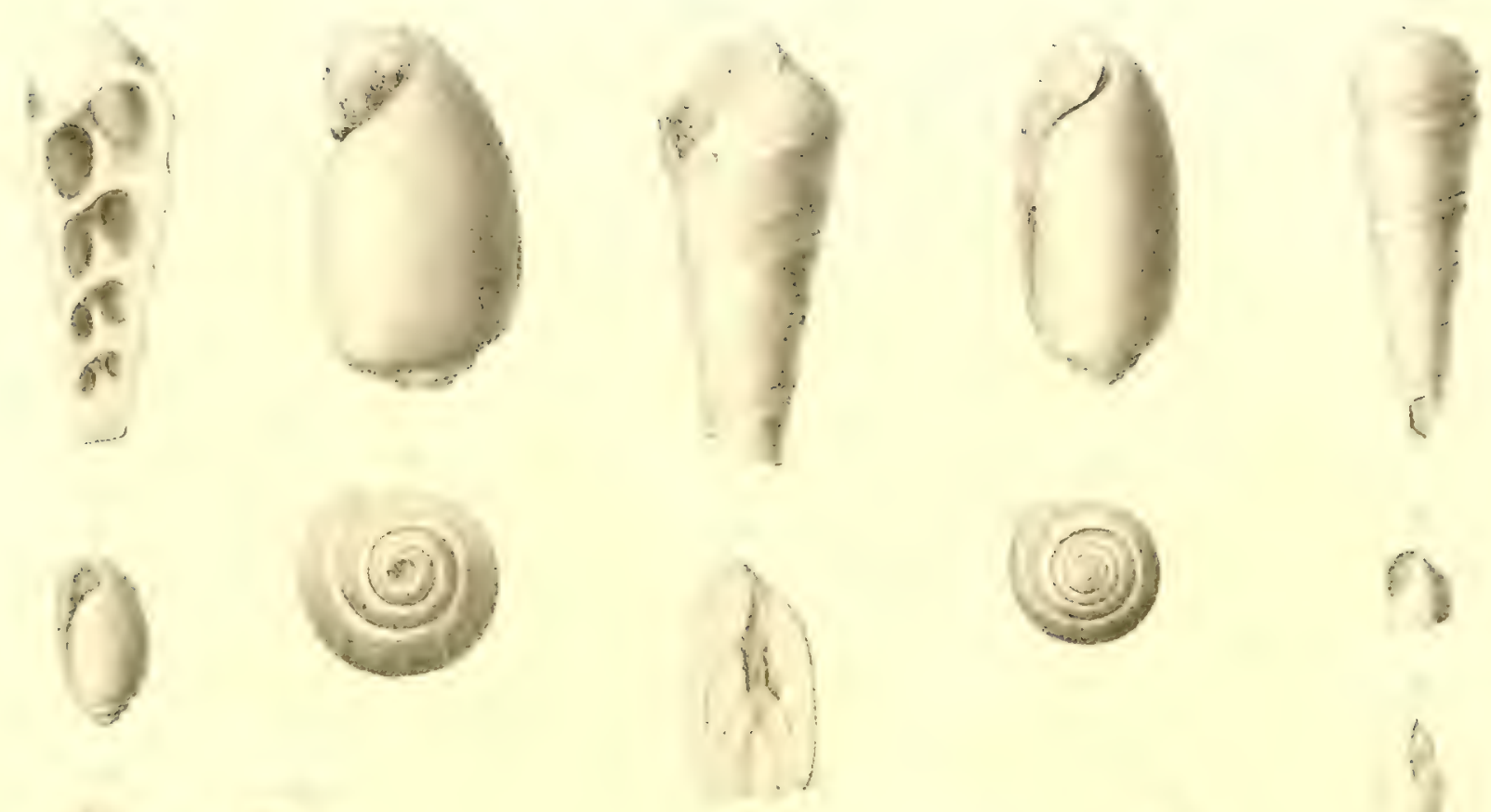

(4)

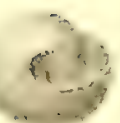
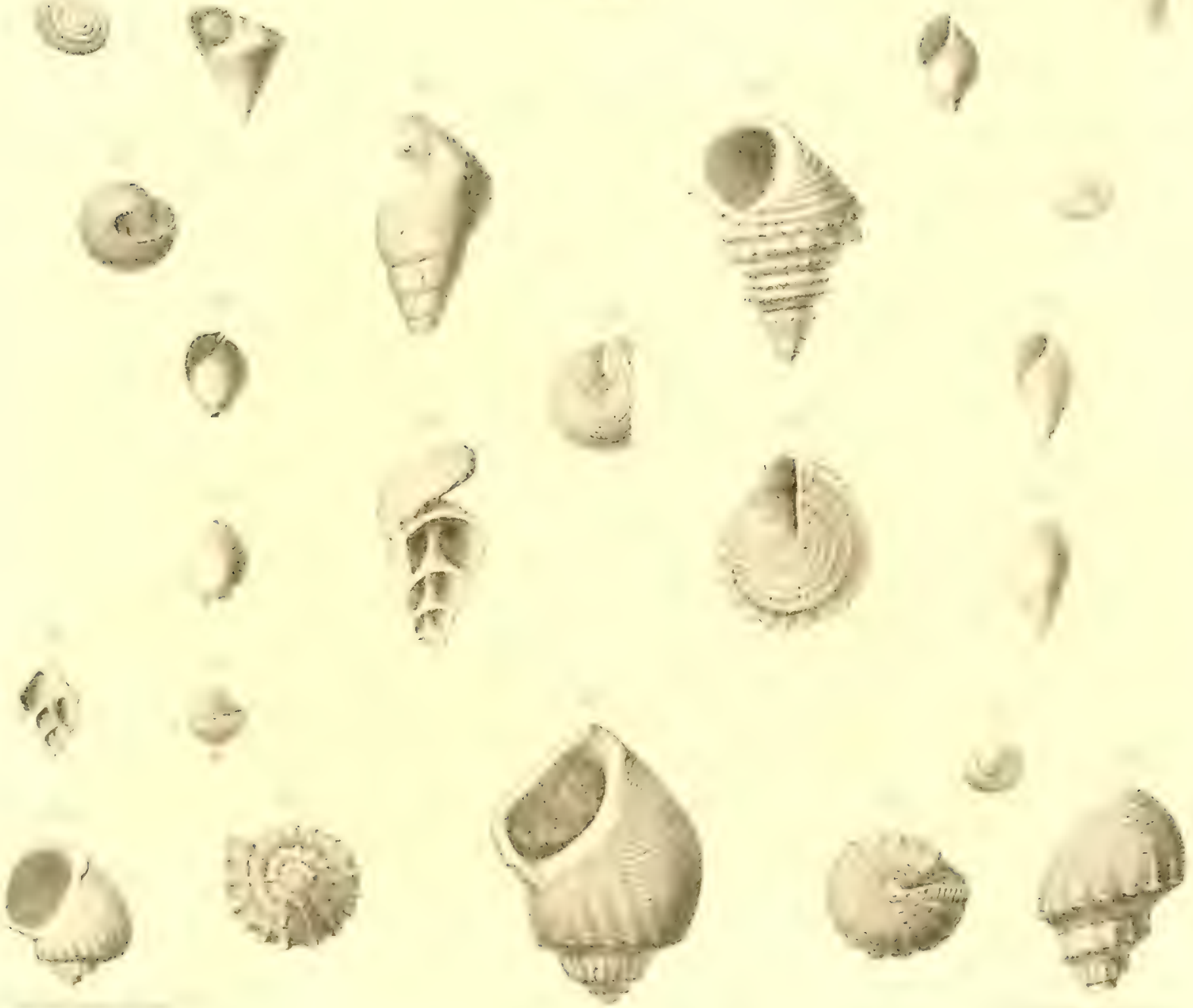

ifrlania viltala 


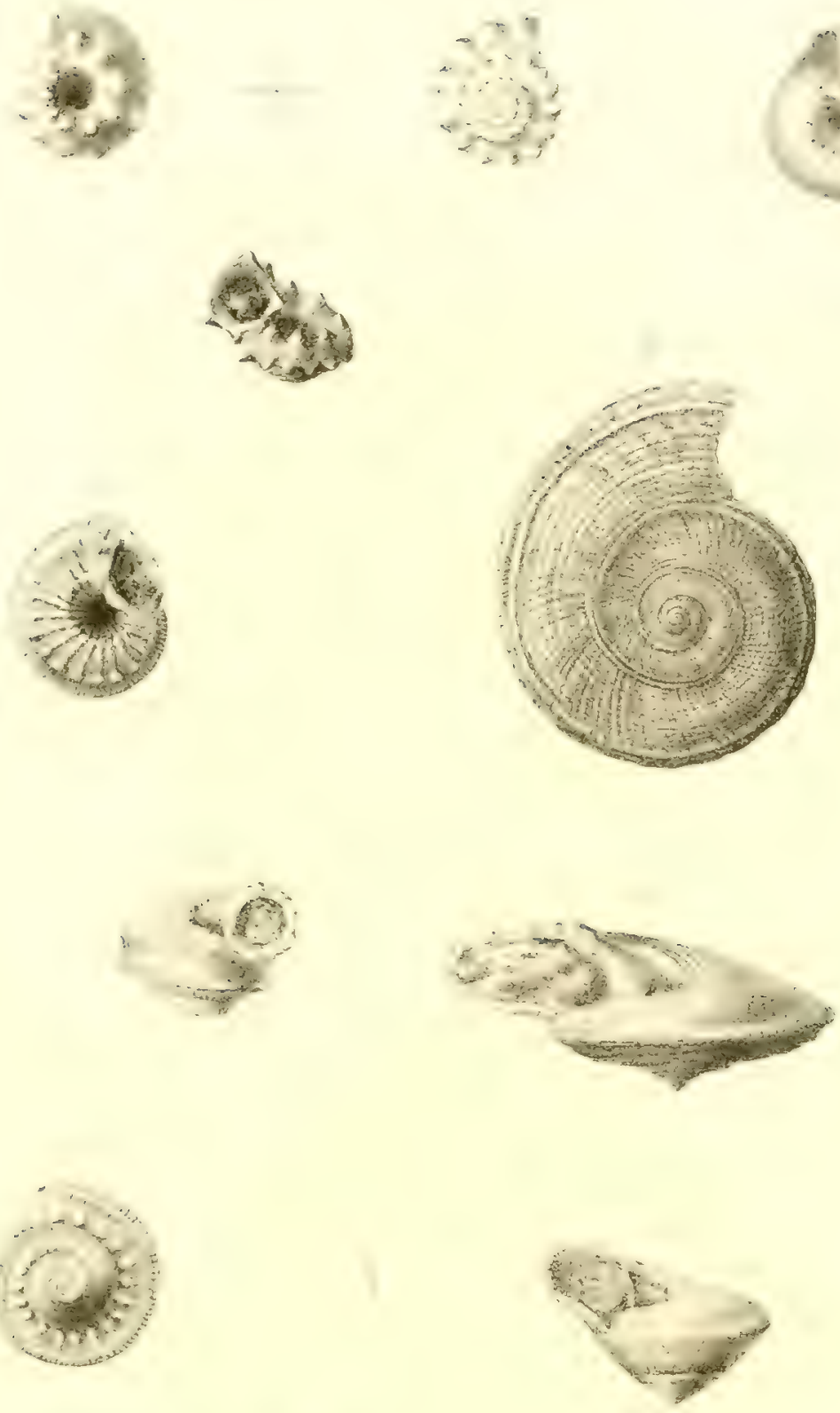
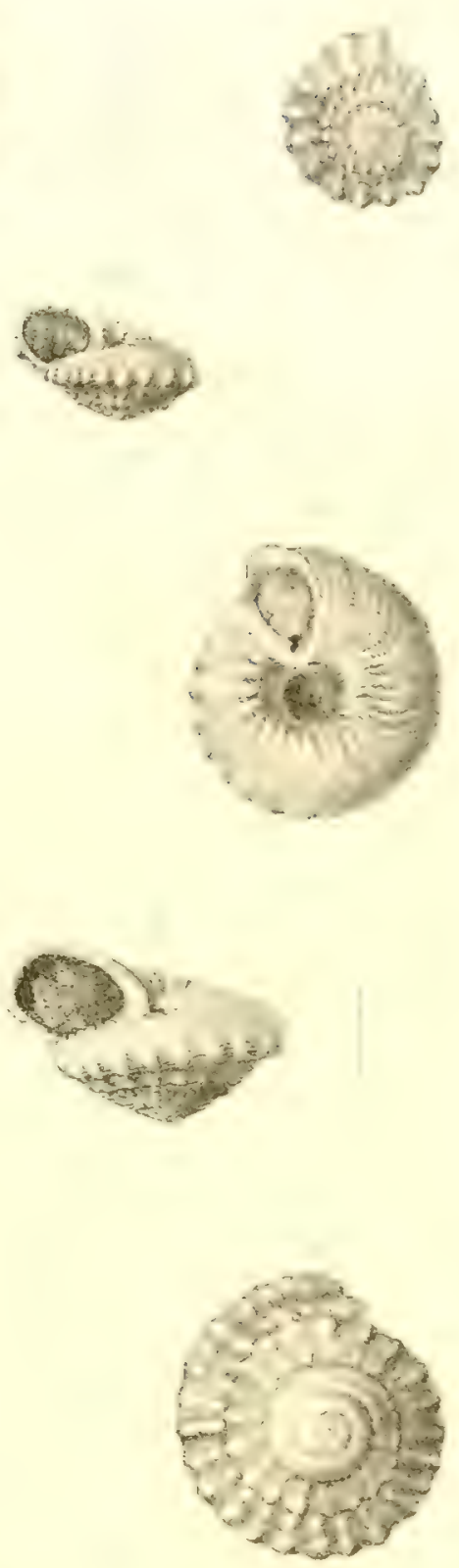

II
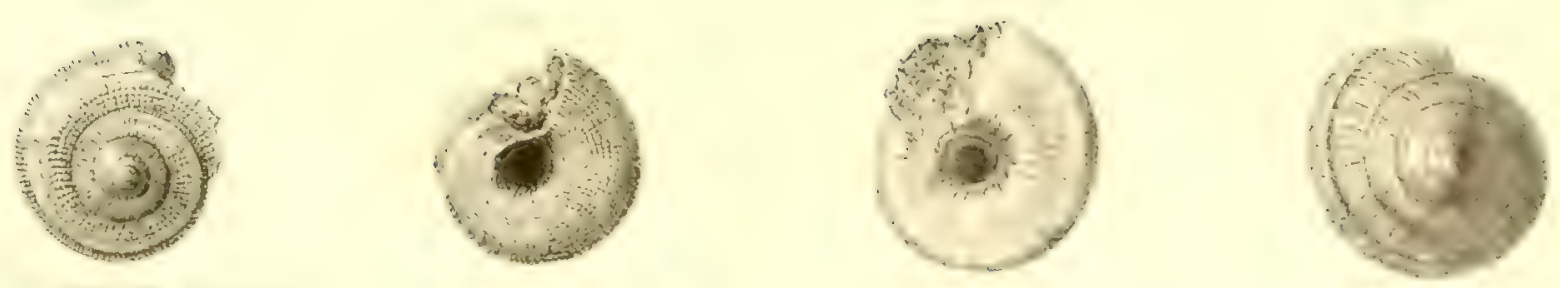


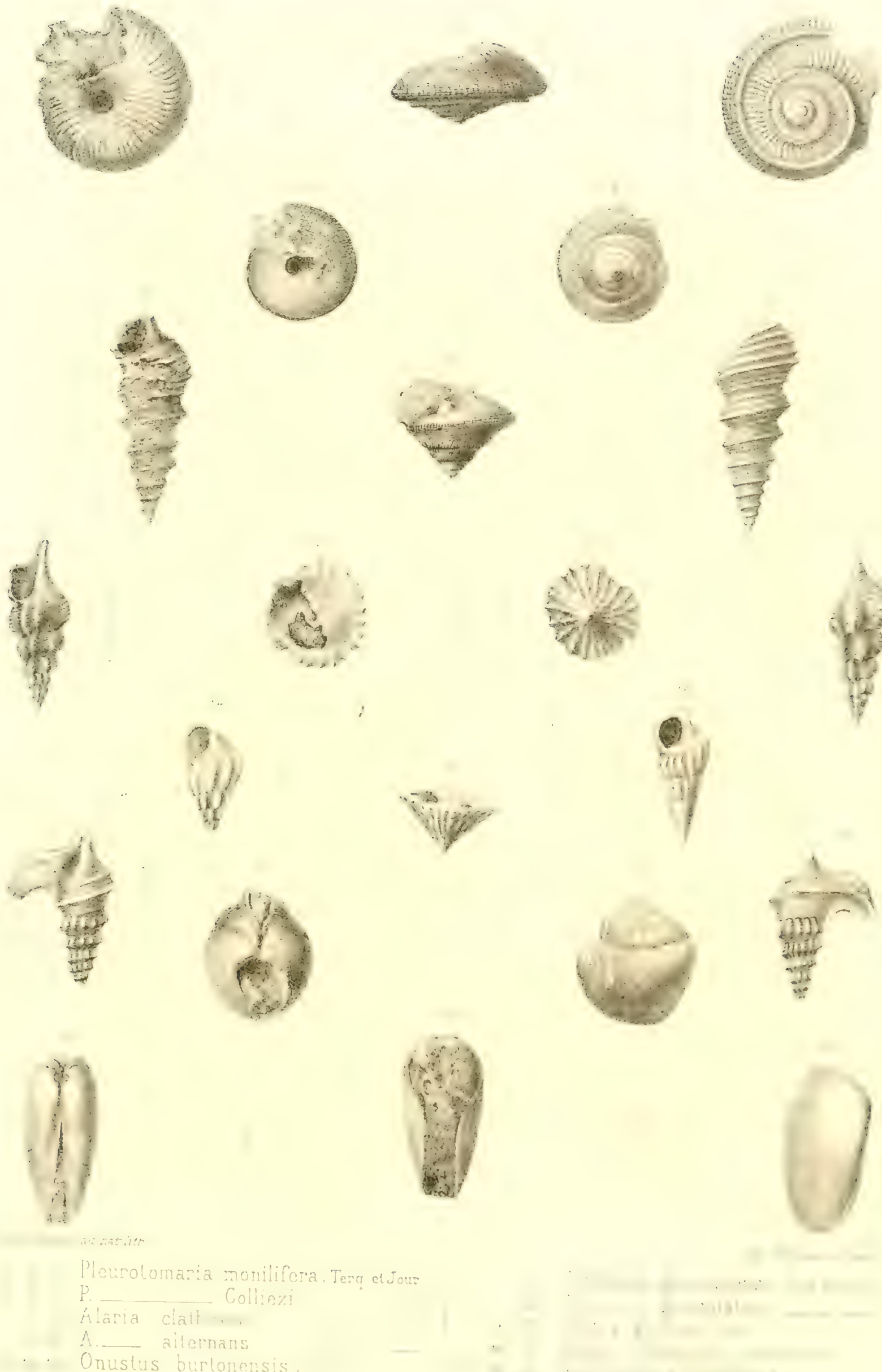

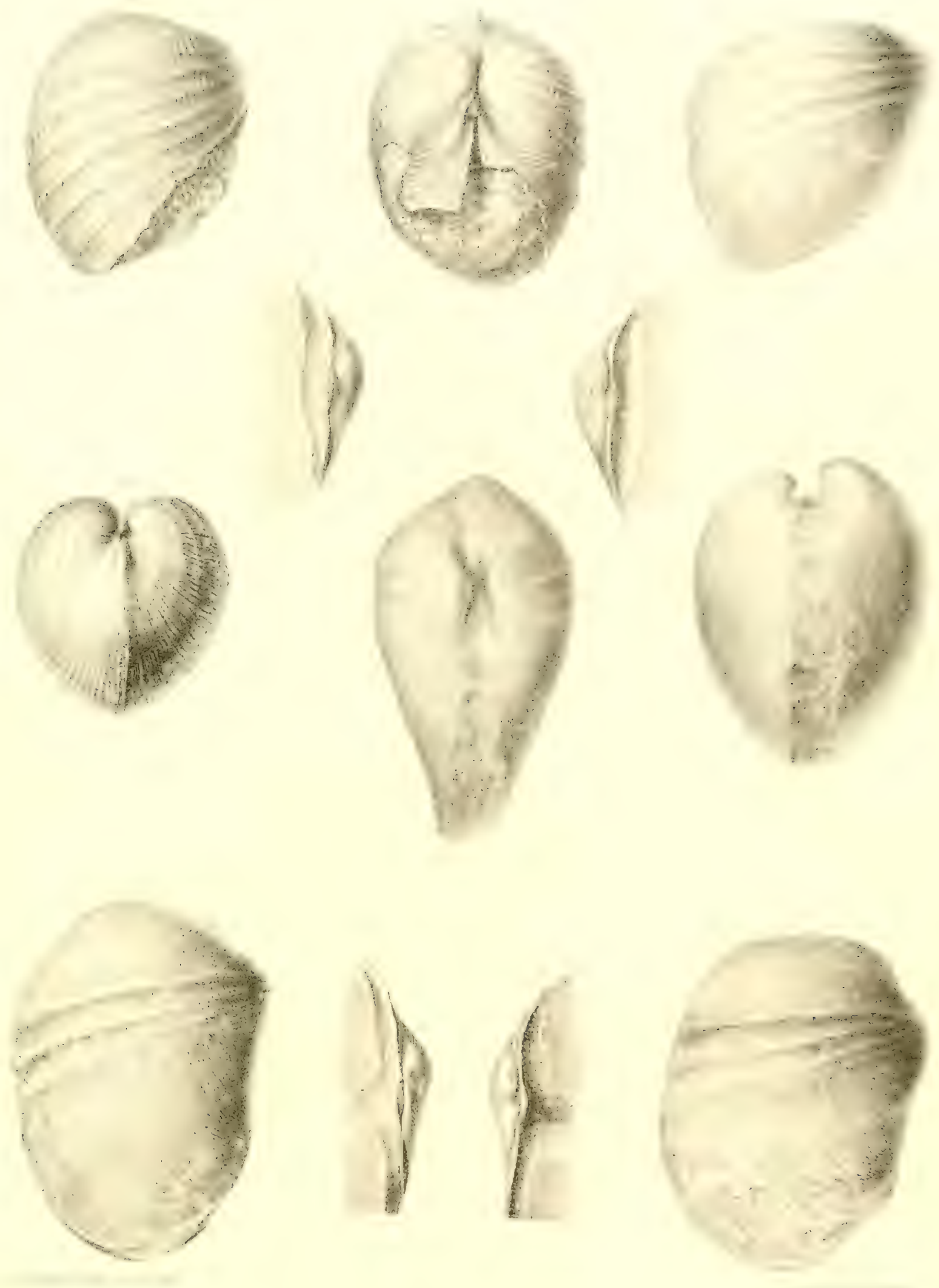


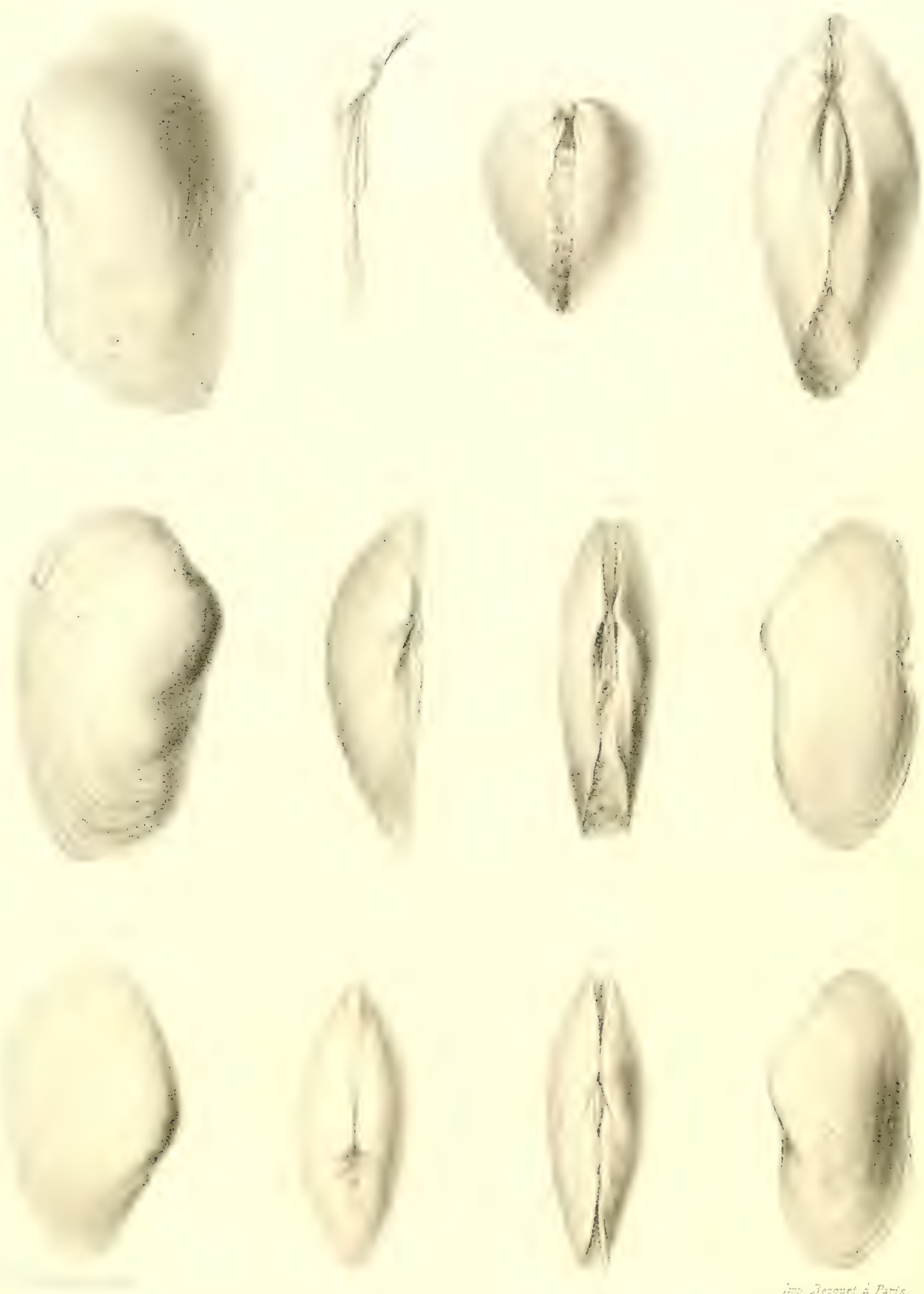



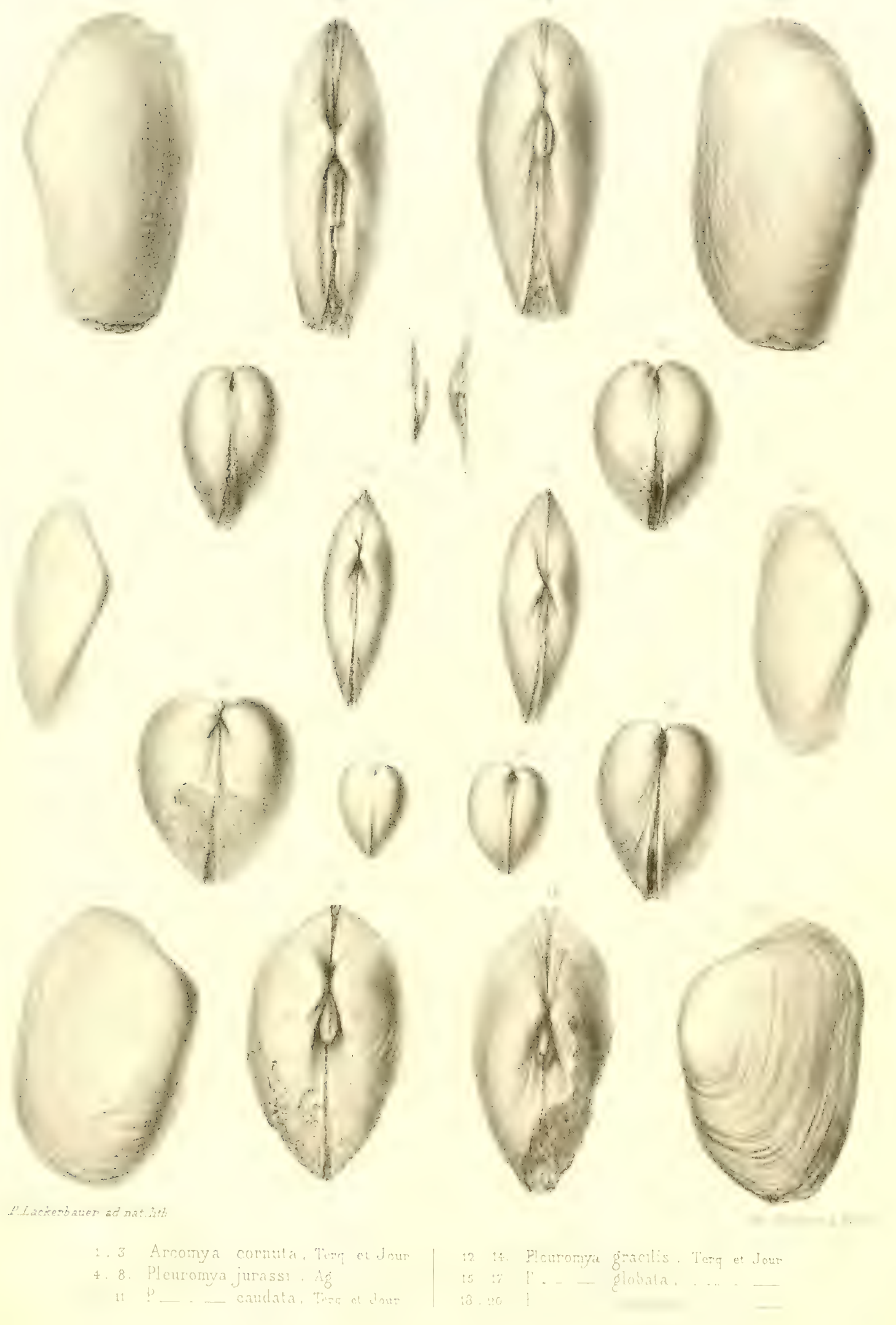




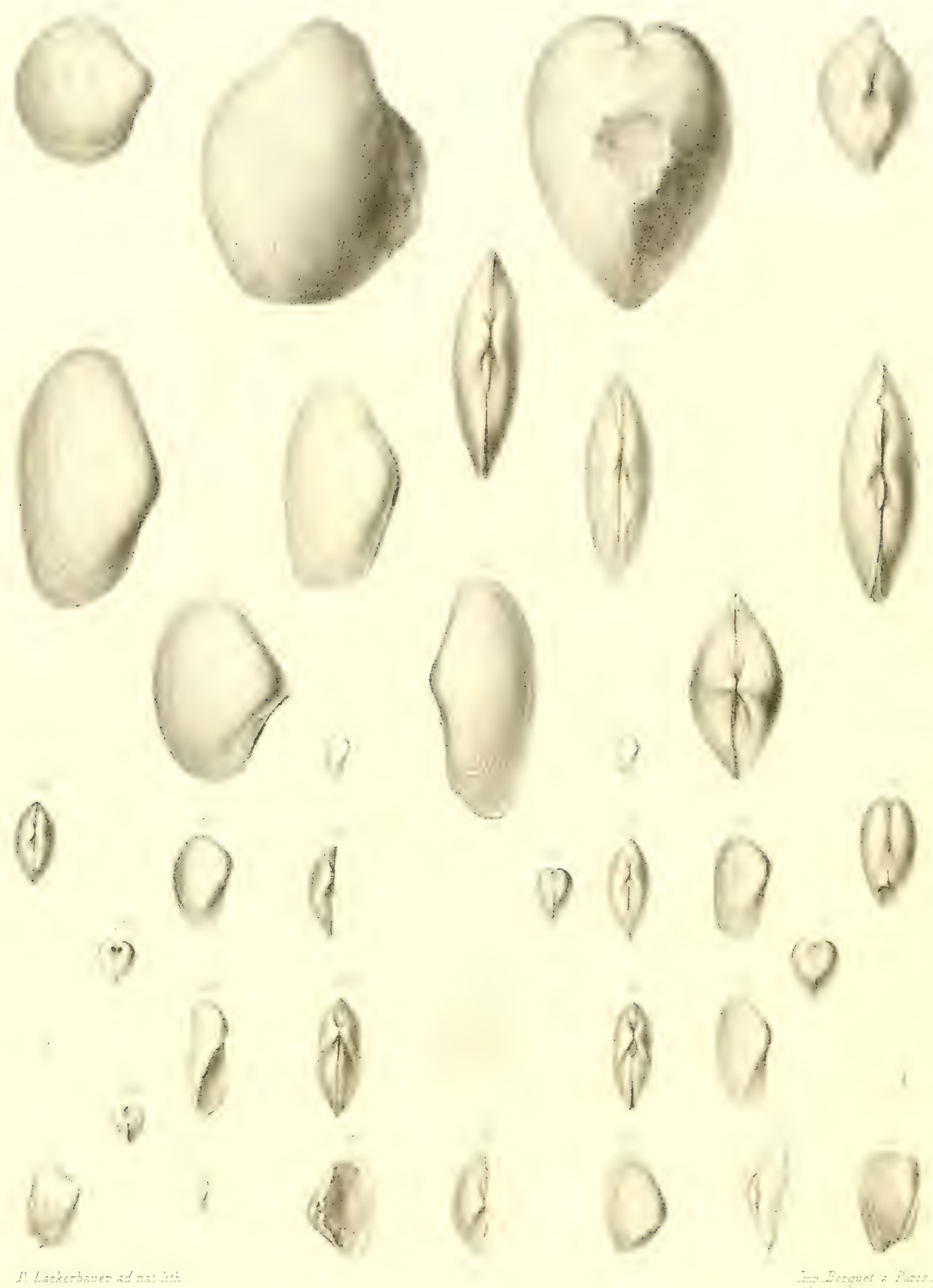




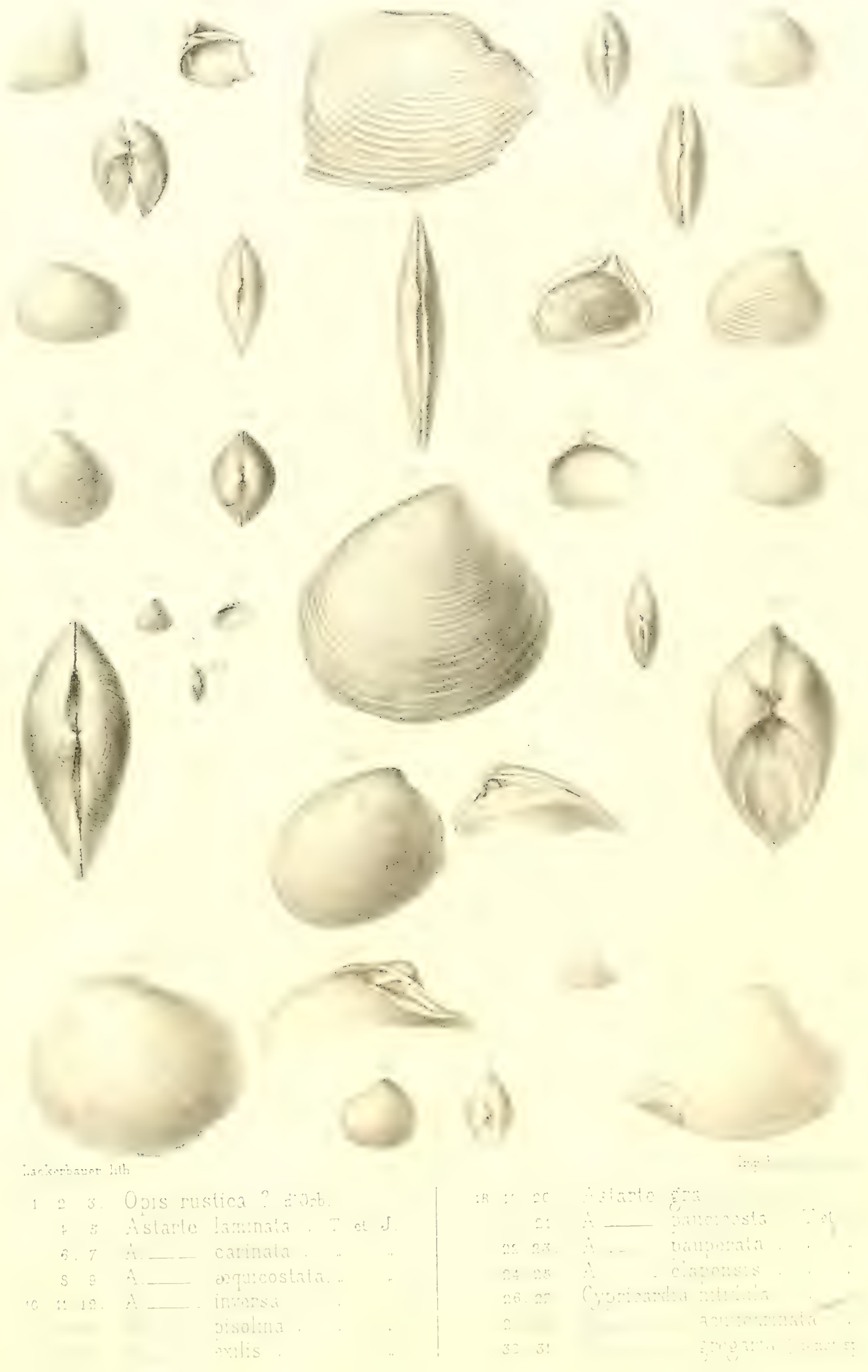


. 

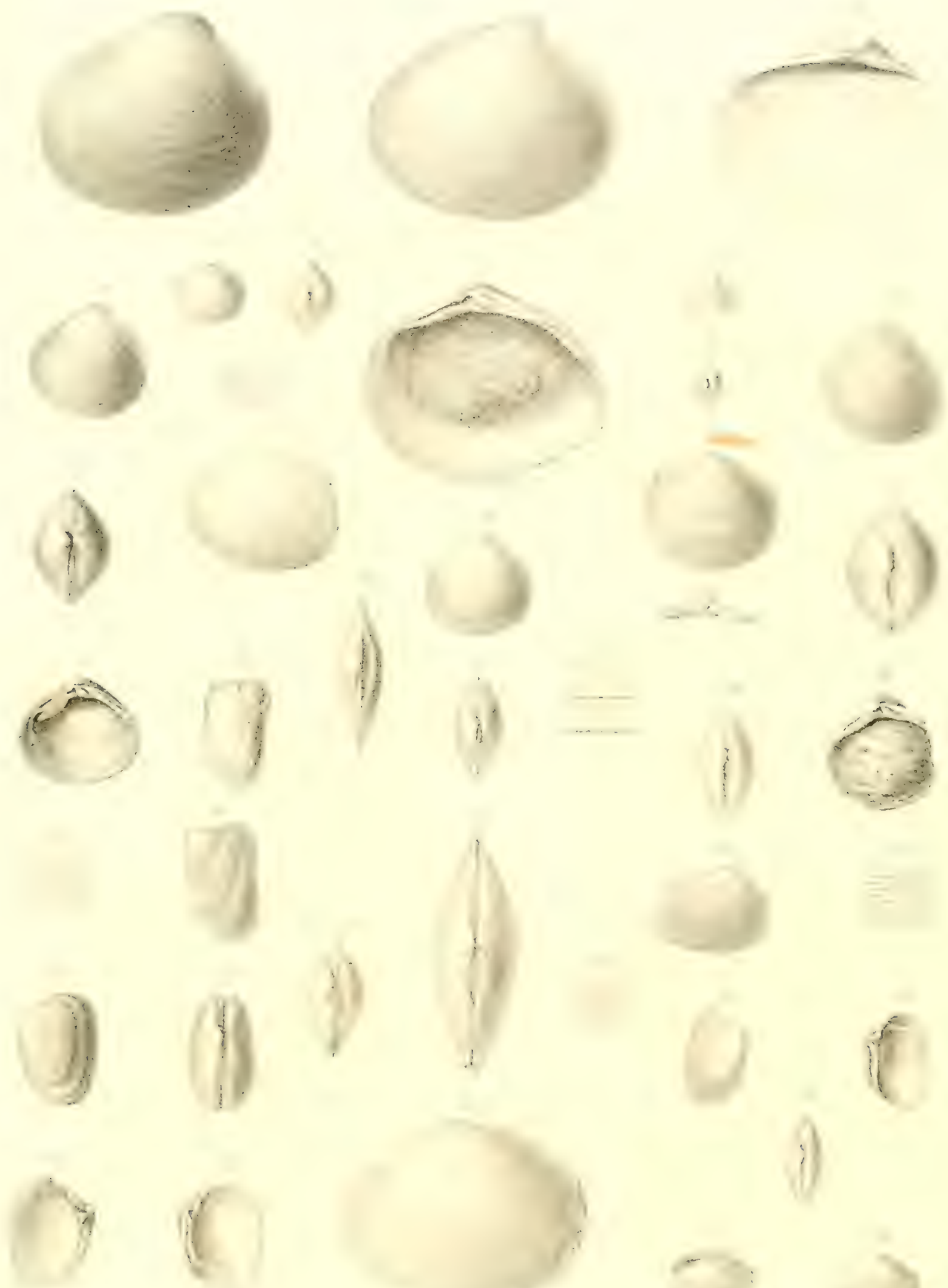<smiles>[2H][CH]</smiles>

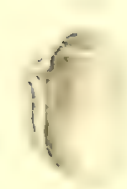




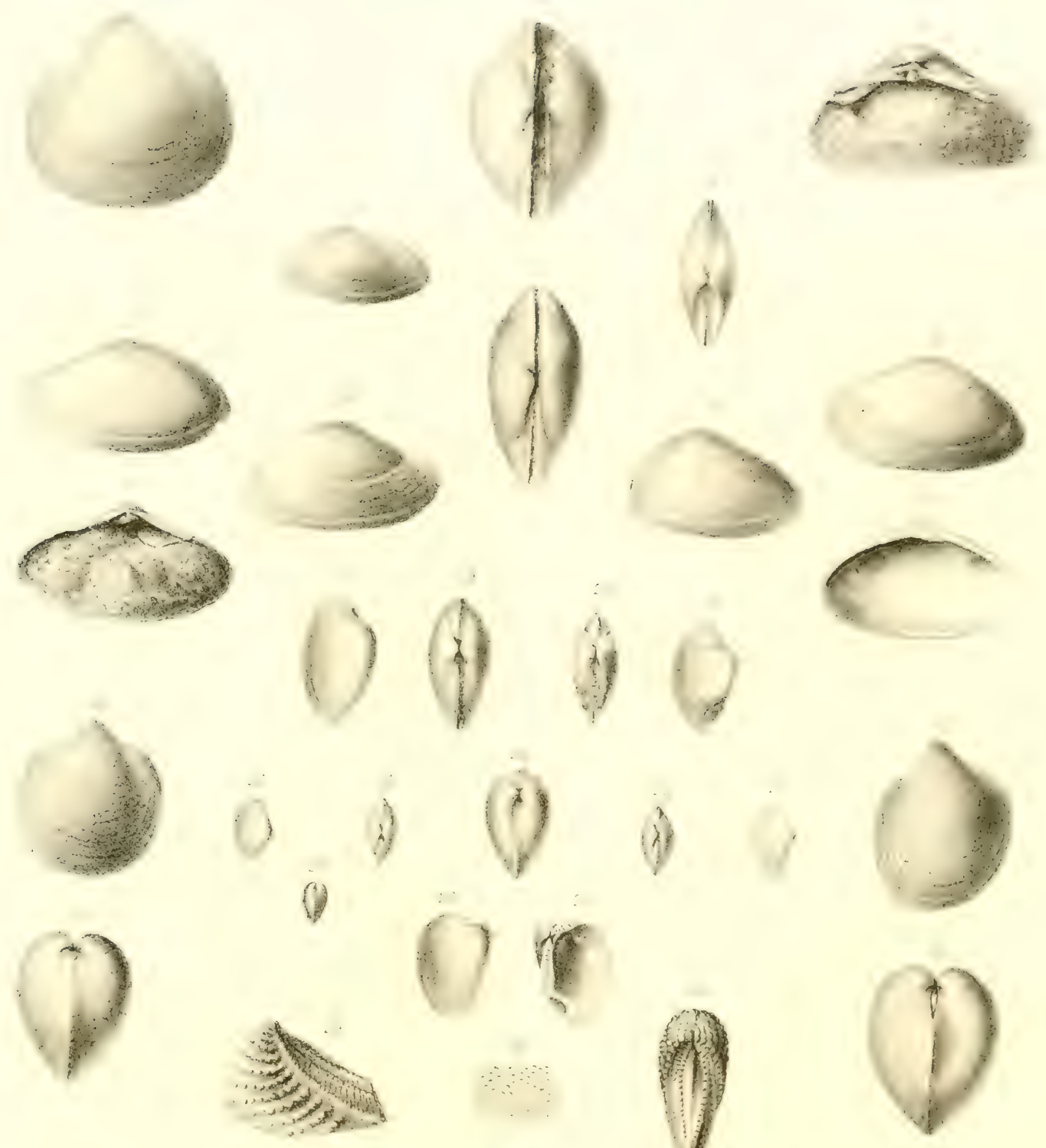

(i)
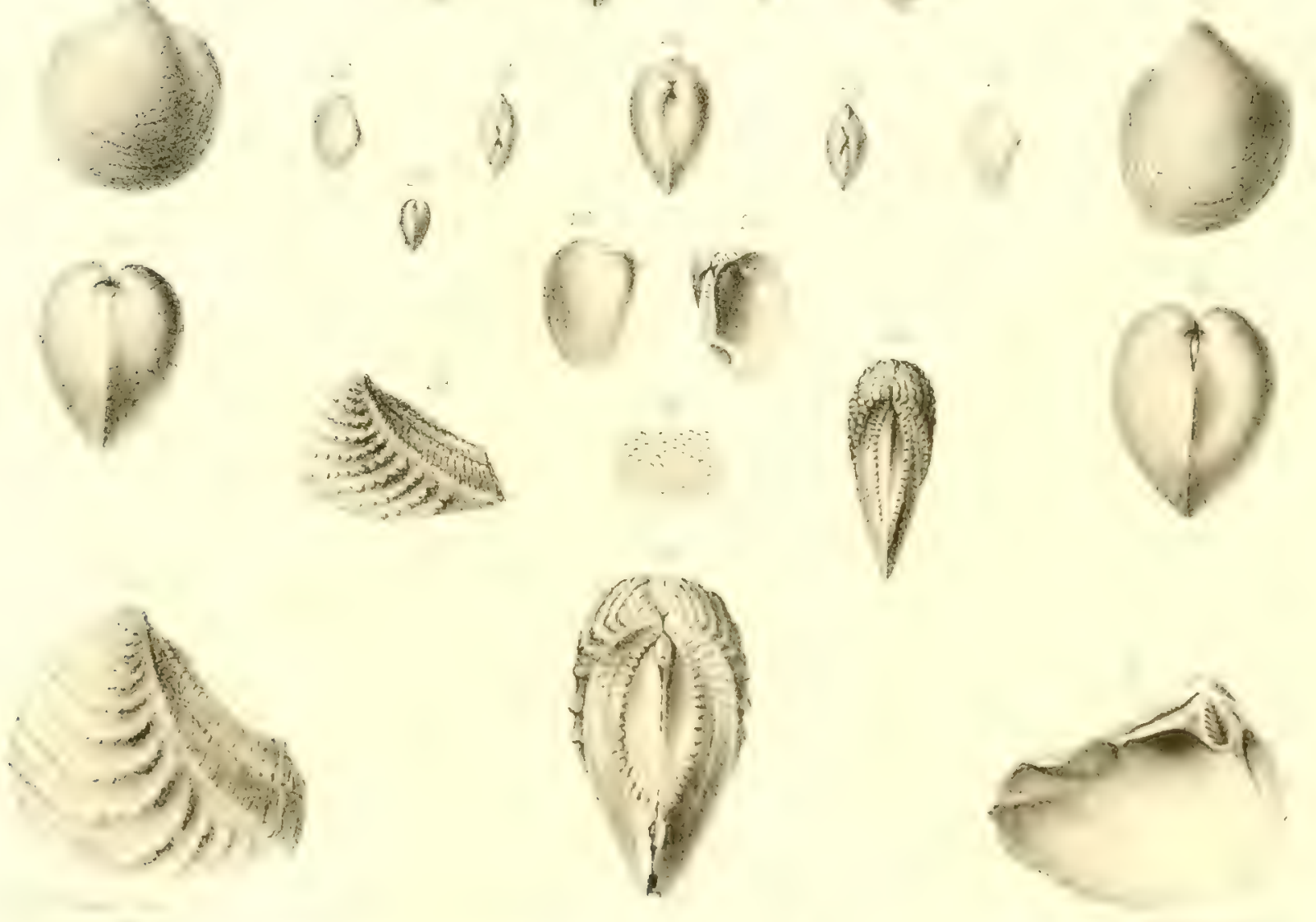



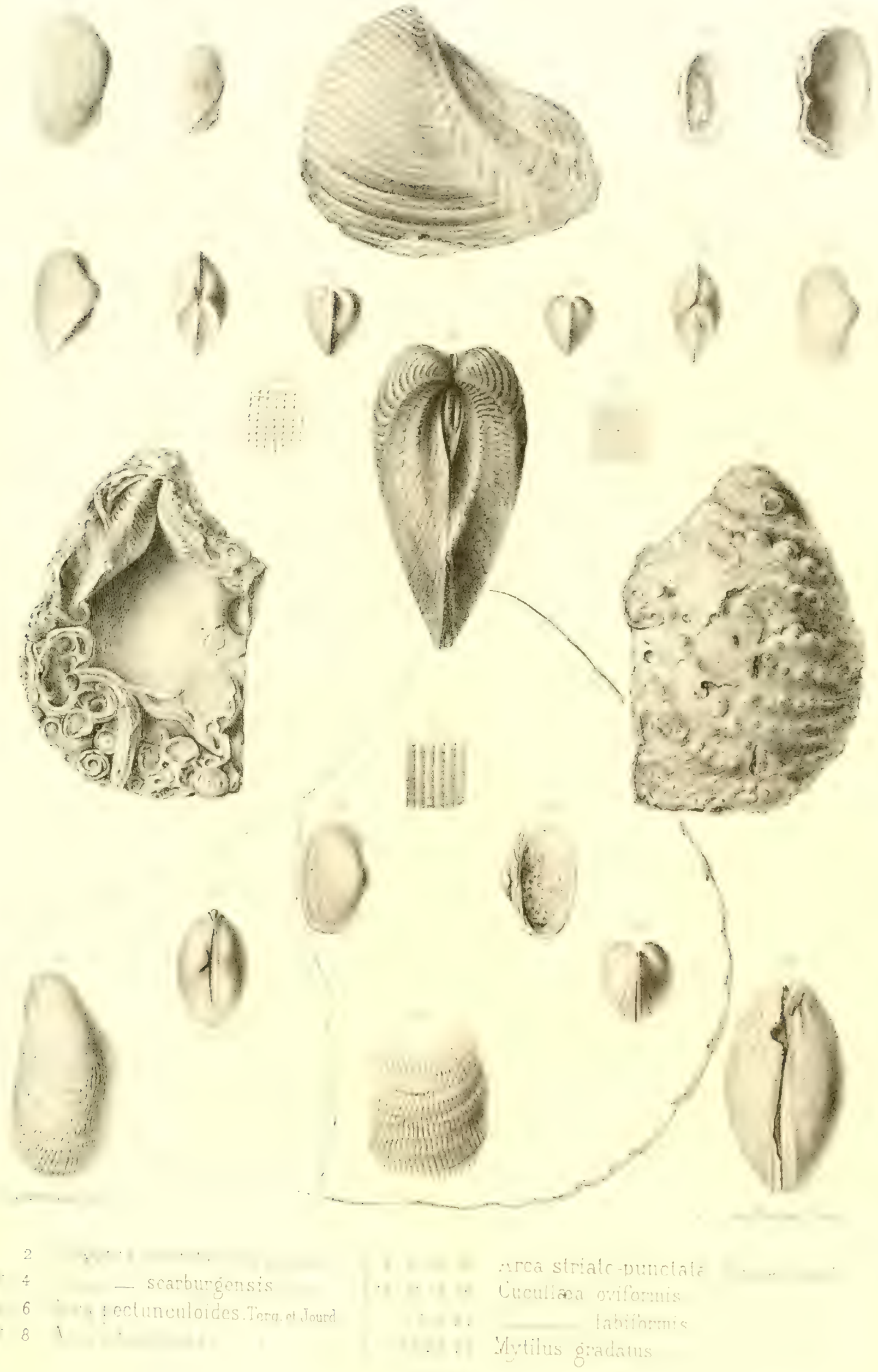




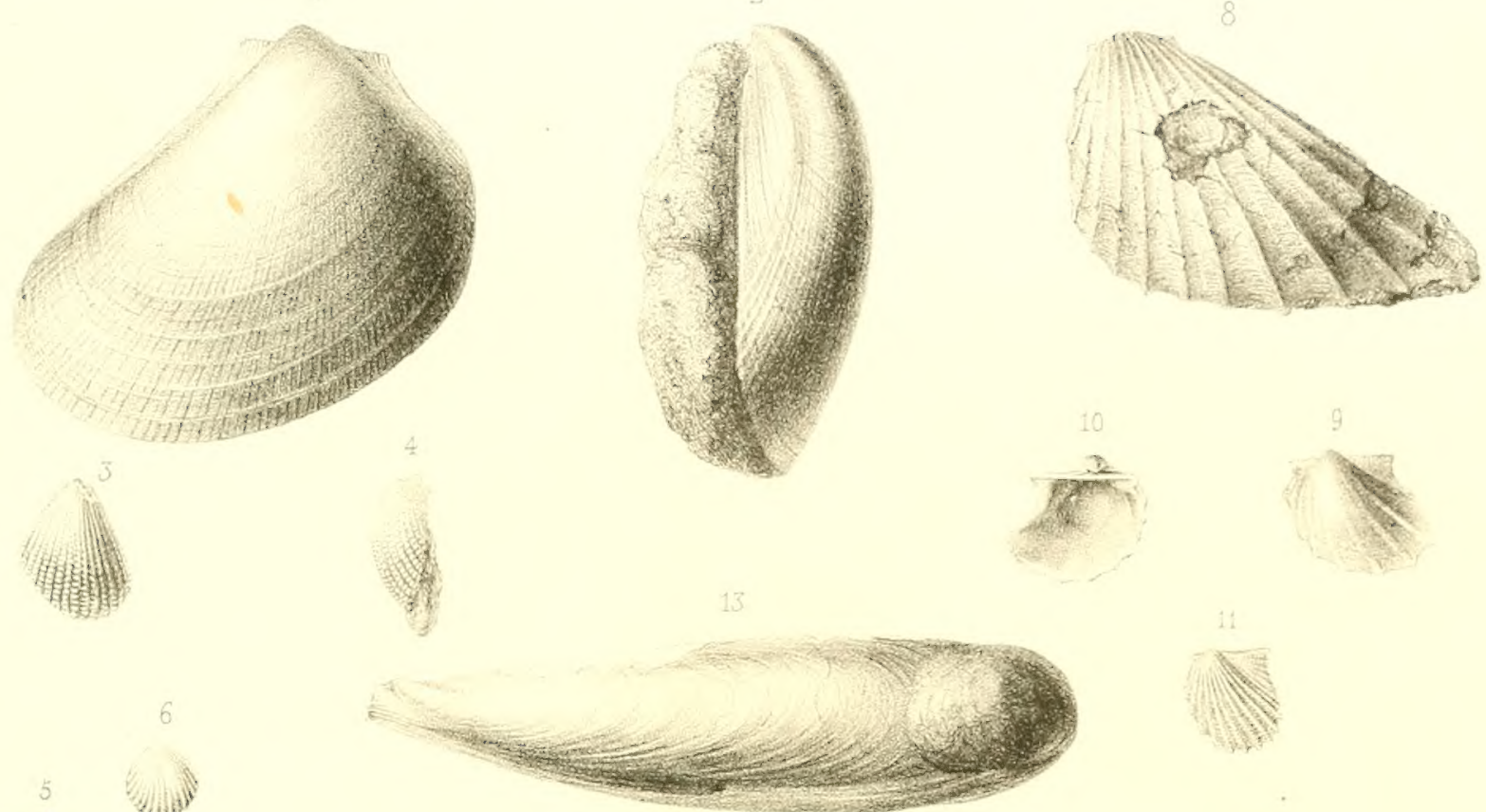

$5 \quad$ wis
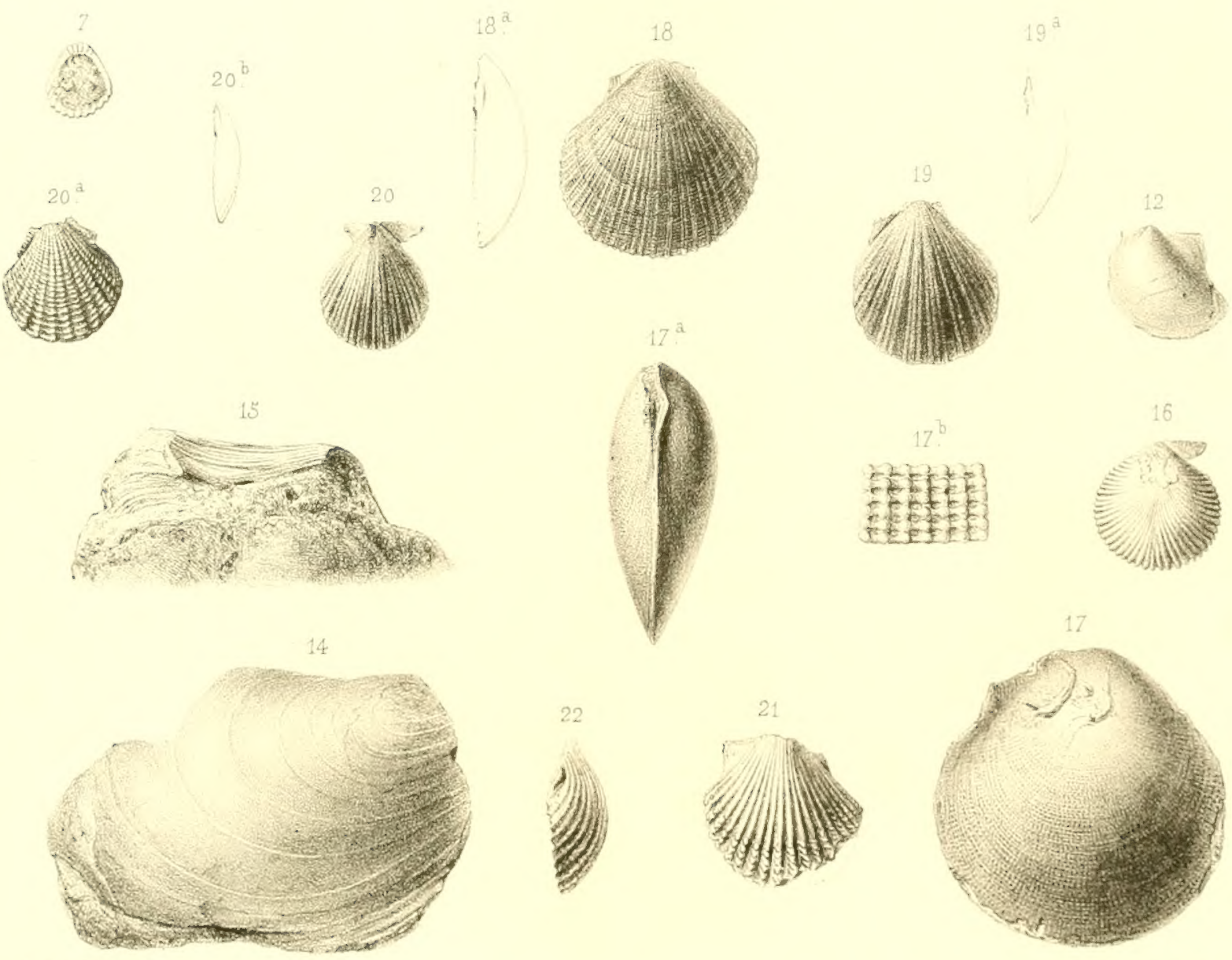

Imp Becquel Paris
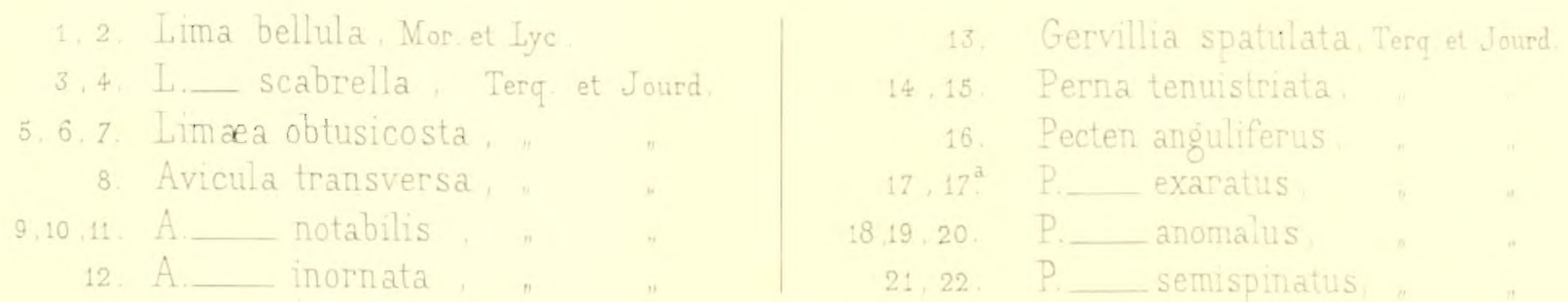

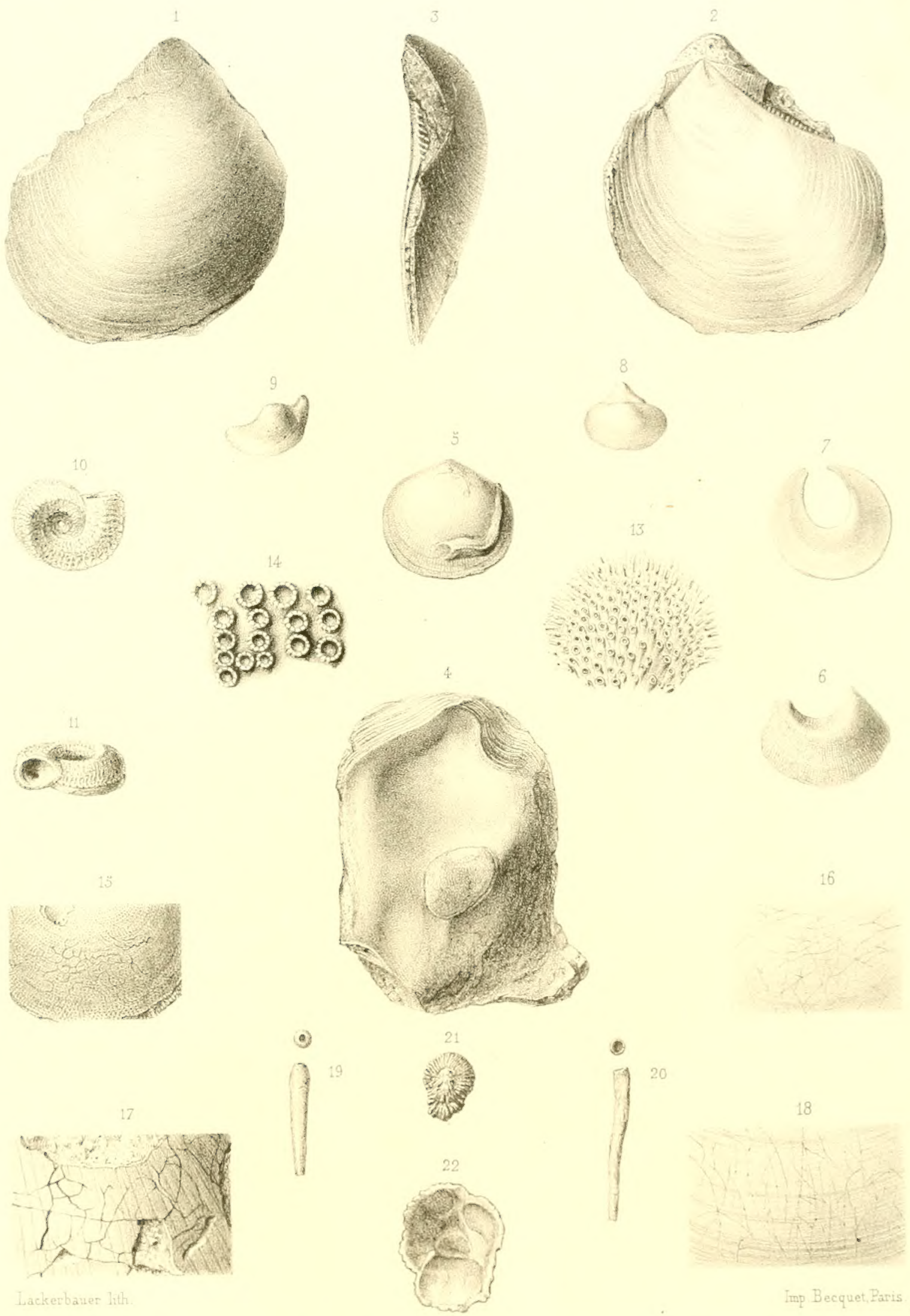

2. 2. 3. Pecten himaformis, Terc et Jourd.

14. Berenicea denticulata, Terq. et Jourd.

Ostrea wiltonensis Lyc

15. Terebripora nierog lyphica

5. Anomia detrita, Terq. et Jourd.

6.7. A. monilifera.

stellifera

8.9. Thecidea gibbosula

latesulcata.

10.11. Serpula scobulina.

radiciformis, 
